\title{
AFDM:
}

\section{An Advanced Fluid-Dynamics Model}

Volume VI: EOS-AFDM Interface

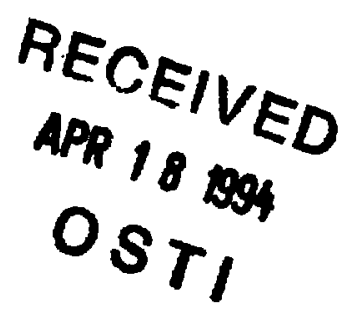

\section{Los Alamos}

Los Alamos National Laboratory is operated by the University of California for the Unifed States Department of Energy under contract W-7405-ENG-36. 
This work was supported by the US Nuclear Regulatory Commission, Accident Evaluation Branch, Division of Systems Research, Office of Nuclear Regulatory Research.

An Affirmative Action/Equal Opportunity Employer

This report zons prepared as an account of urork sponsored by an agency of the

United Staie's Govermment. Neither The Regents of the University of California, the

United States Government nor any nency thereof, nor any of their employes, makes any twarranty, express or implied, or assumes any legal liability or responsibility for the sccuracy. completeness, or usefulness of any information, apparatus, product, or process disclosed, or represents that its use would not infringe privately ooned rights. Reference hercin to sny specific commercial product, process, or service by trade name, trademark, manufacturer, or otherwise, does not necessarily consfitute or imply its endorsement, recommendation, or faroring by The Regents of the University of California, the United States Government, or any agency thercof. The vieus and opinions of aullors expressed herein do not necessarily state or reflect thosi' of The Regents of the University of California, the United States Government, or any ngency thereof. 
AFDM: An Advanced Fluid-Dynamics Model

Volume VI: EOS-AFDM Interface

Compiled by

G. Henneges*

S. Kleinheins*

Contributors

W. R. Bohl

E. A. Fischer

L. Goutagny

P. J. Maudlin

H. Ninokata

F. R. Parker

D. Wilhelm

"INR, Kernforschungszentrum Karlsruhe, Pustfach 3640, D-7500 Karlsruhe 1, GERMANY 
PREFACE

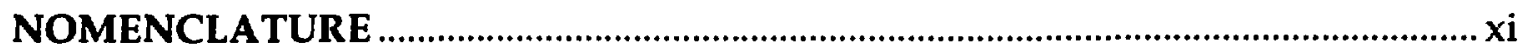

ABSTRACT

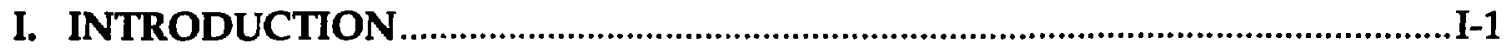

A. Thermodynamic vs Mechanical Equilibrium ...................................................

B. Multiphase-Multimaterial Systems...................................................................

C. Simplified Analytic EOS vs Precise Tabular EOS (TEOS).................................

D. EOS Implementation in the Code...................................................................

E. Thermophysical Properties...................................................................................

II. THE SIMPLIFIED ANALYTIC EQUATION OF STATE (SAEOS) ........................I-1

A. Basic Assumptions .................................................................................................

B. Functions Provided by the SAEOS......................................................................

1. Internal Energies and Enthalpies ............................................................-3

2. Microscopic Densities and Pressures ........................................................

3. Saturation Temperatures.......................................................................II-8

C. Pressure Iteration Derivatives ...................................................................12

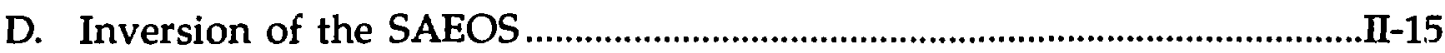

E. Step 1 Derivatives.............................................................................................16

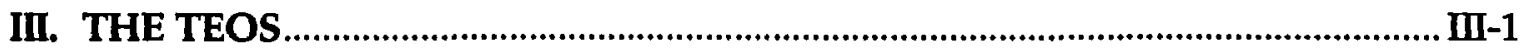

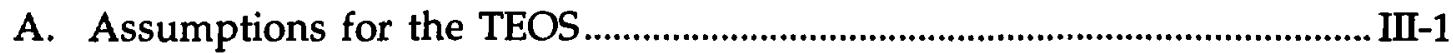

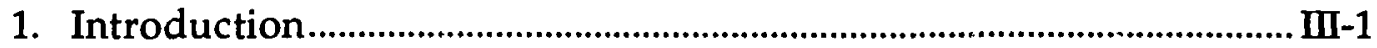

2. Composition of the TEOS ........................................................................... III-1

3. Usage of the TEOS Data ................................................................................. III-2

4. Usage of Other EOS Data ............................................................................ III-5

B. Multimaterial Multiphase EOS Interface Model ............................................ III-6

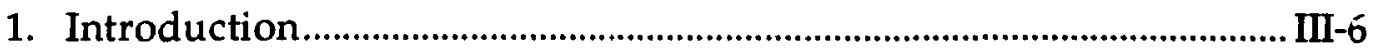

2. Material Equations of State .......................................................................... III-9

3. Mechanical Equilibrium............................................................................ III-13

4. Saturation Properties ............................................................................... III-33

5. Temperature Inverse Calculations ....................................................... III-34

6. EOS Function Partial Derivatives ......................................................... III-44

C. The TEOS Property Derivatives................................................................ III-54

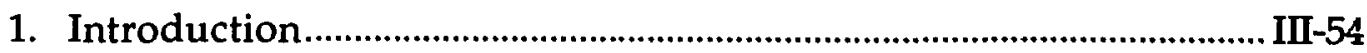




\section{CONTENTS (CONT.)}

\section{Page}

2. Derivative Dependencies...................................................................... III-54

3. Volume Fraction Derivatives................................................................. III-55

4. Other Derivatives ..................................................................................... III-73

D. The TEOS Interface Routines...................................................................... III-84

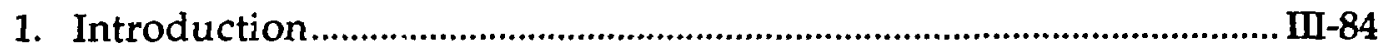

2. Initialization Routines .................................................................... I[i-84

3. Mechanical Equilibrium Routine EOSPEQ and Related Routines... III-84

4. Temperature Inversion Routine EOST and Related Routines......... III-92

5. Routines Calculating Derivatives............................................................ III-92

E. The TEOS Access, Search, and Interpolation Routines............................. III-103

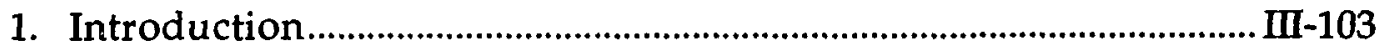

2. The SESAME EOS Data Library............................................................... III-103

3. Other EOS Data Tables ............................................................................ III-107

4. Storage of the Data Tables .......................................................................... III-107

5. Tables Search and Interpolation.............................................................. III-108

6. Initialization Routines SESINI, SPNINI, and SESPRT .......................III-112

7. Access Routines SESEOS, VSTOP, VSPOT, VSELOT, VSTOEL, and SPINDM.......................................................................................1112

8. EOSPAC Search and Interpolation Routines........................................III-112

9. Search and Interpolation Routines LAGRAN and URANUS........ III-112

IV. THERMOPHYSICAL PROPERTIES ..............................................................

A. Surface Tension...................................................................................................

1. General Form of the Surface Tension Correlation...................................IV-1

2. Surface Tension Function......................................................................IV-2

B. Vapor Thermophysical Properties..........................................................IV-2

1. Heat Capacity at Constant Pressure............................................................

2. Vapor Dynamic Viscosity.....................................................................

3. Vapor Thermal Conductivity …...........................................................IV-3

4. Vapor Mixture Rules .................................................................................

5. Second-Order Correlation for the Thermal Conductivity.....................IV-4

6. Mixture Heat Capacity...............................................................................

C. Solid and Liquid Thermophysical Properties..................................................

1. Liquid Dynamic Viscosity .........................................................................IV-5 


\section{CONTENTS (CONT.)}

2. Solid and Liquid Thermal Conductivity ................................................IV-5

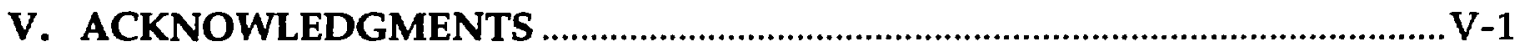

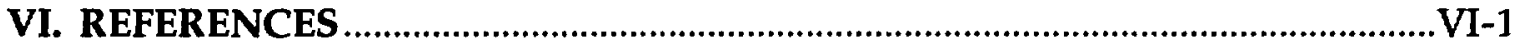

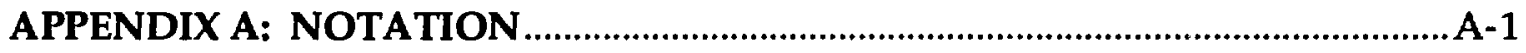

APPENDIX B: ROUTINE SPECIFICATIONS ….........................................................

APPENDIX C: ROUTINE FLOWCHARTS * ........................................................

APPENDIX D: VARIABLE NAMES AND SYMBOLS ........................................ D-1

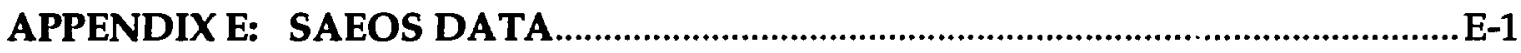

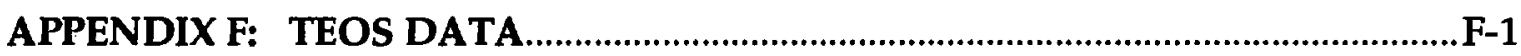

APPENDIX G: THERMOPHYSICAL PROPERTIES, DATA, AND PLOTS.............. G-1

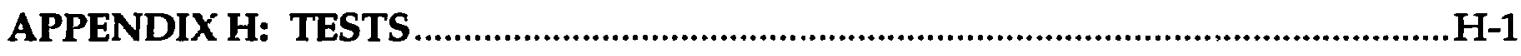

APPENDIX I. CODE CORRECTIONS....................................................................

"This appendix is not contained within this volume but forms a separate document, which is available upon request. 


\section{PREFACE}

Volume VI of the Advanced Fluid-Dynamics Model (AFDM) documentation was compiled by the editors of this volume in September 1988 and distributed as a ready-for-print copy in October 1988. It was based on the AFDM $Y$ version of September 9, 1988, plus a supplementary set of corrections for the implementation of a solid substance tabular equation of state (EOS) and for some other minor changes intended to be exported along with the $Y$ source.

Unfortunately, the AFDM versions exported by Los Alamos National Laboratory (LANL) on November 3, 1988 (the preliminary $Z$ version), and on January 10, 1989 (the final $Z$ version), included a substantially modified set of corrections such that code and Vol. VI documentation were no longer in congruence. Contrary to an agreement within the AFDM team, the LANL staff responsible for the modifications never supplied the documentation updating Vol. VI to the status of the exported Z version. To terminate the business in a satisfactory way, one of the compilers of this volume (S. Kleinheins, KfK) did the updating during the revision of the documentation for the final LANL editing effort in September/October 1991. It must be understood that $\mathrm{KfK}$ is in no way responsible for the delayed issue of Vol. VI.

As noticed in September 1988 already, the AFDM Z version coding differs slightly from the correct specification of the TEOS-EOSAT routine and from the correct phase state branchings in the EOSPEQ and TEOS-EOST routines, as documented in Tables I, II, and III, respectively, of Chap. III.D. To make the code agree with the documented specifications, some corrections in the coding are necessary. Further, for a somewhat lucid presentation in the updated documentation, the description of the tabular EOS substance and material assignment in the code modification had to be based on default values of some input quantities different from those in the exported code. The code corrections for this change, as well as for the former ones, rendering the AFDM Z and AA coding identical with the updated Vol. VI documentation, are supplied in App. I. 


\section{NOMENCLATURE}

A complete and unique nomenclature list is difficult to produce when first, different authors with their own styles wrote such a volume and second, when the limited alphabets available lead either to duplication or awkward, lengthy symbols. The larger the list, the greater the difficulty. If a subscript or superscript occurs only once or is required for clarity, it is included with the symbol. The notation used for the symbols and subscripts follows the AFDM nomenclature in most respects. A more detailed description is given in App. A, Table A.I; and App. D, Table D.II.

$c_{\mathrm{VG}, \mathrm{M}} \quad$ Vapor material heat capacity at constant volume $[\mathrm{J} /(\mathrm{kg}-\mathrm{K})]$

$c_{\mathrm{vL}, \mathrm{M}} \quad$ Liquid material heat capacity at constant volume $[\mathrm{J} /(\mathrm{kg}-\mathrm{K})]$

$c_{\mathrm{vS}, \mathrm{M}} \quad$ Solid material heat capacity at constant volume $[\mathrm{J} /(\mathrm{kg}-\mathrm{K})]$

$c_{\mathrm{p}} \quad$ Specific heat capacity at constant pressure $[\mathrm{J} /(\mathrm{kg}-\mathrm{K})]$

$c_{m} \quad$ Velocity of sound for density components $m[\mathrm{~m} / \mathrm{s}]$

$\mathrm{C}_{\mathrm{KAG}, \mathrm{M}}$ Parameter in thermal conductivity relations $[\mathrm{W} /(\mathrm{m}-\mathrm{K})]$

$\mathrm{C}_{\mathrm{KBG}, \mathrm{M}}$ Parameter in thermal conductivity relations $\left[\mathrm{W} /\left(\mathrm{m}-\mathrm{K}^{2}\right)\right]$

$\mathrm{C}_{\mathrm{KCG}, \mathrm{M}}$ Parameter in thermal conductivity relations $\left[\mathrm{W} /\left(\mathrm{m}-\mathrm{K}^{3}\right)\right]$

CKAm Parameter in thermal conductivity relations $[\mathrm{W} /(\mathrm{m}-\mathrm{K})]$

$\mathrm{C}_{\mathrm{KBm}}$ Parameter in thermal conductivity relations $\left[\mathrm{W} /\left(\mathrm{m}-\mathrm{K}^{2}\right)\right]$

CMU AM Parameter in dynamic viscosity relations $[\mathrm{kg} /(\mathrm{m}-\mathrm{s})]$

$\mathrm{C}_{\mathrm{MU} B, \mathrm{M}}$ Parameter in dynamic viscosity relations [K]

$\mathbf{e}_{\mathbf{m}}$ Specific internal energy of component $\mathrm{m}[\mathrm{J} / \mathrm{kg}]$

eCon,M Liquid condensate specific internal energy $[\mathrm{J} / \mathrm{kg}]$

eCrt,M Critical specific internal energy [J/kg]

eLiq,M Liquidus specific internal energy $[J / \mathrm{kg}]$

esId,M Solic: EOS specific internal energy $[\mathrm{J} / \mathrm{kg}]$

eSol,M Solidus specific internal energy $[\mathrm{J} / \mathrm{kg}]$

eSus, $M \quad$ Solid specific internal energy at sublimation $[\mathrm{J} / \mathrm{kg}]$

eVan,M Van-der-Waals type specific internal energy $[\mathrm{J} / \mathrm{kg}]$

eVap,M Saturated vapor specific internal energy $[\mathrm{J} / \mathrm{kg}]$

h Heat-transfer coefficient $\left[\mathrm{W} /\left(\mathrm{m}^{2}-\mathrm{k}\right)\right]$

hGLm Heat-transfer coefficient between vapor and liquid $\mathrm{m} /$ vapor interface $\left[\mathrm{W} /\left(\mathrm{m}^{2}-\mathrm{k}\right)\right]$

hGP Vapor-side heat-transfer coefficient for the structure/vapor interface [W/(m²-k)] 


\begin{tabular}{|c|c|}
\hline $\mathrm{h}_{\mathrm{LmG}}$ & $\begin{array}{l}\text { Heat-transfer coefficient between liquid } m \text { and the liquid } m / \text { vapor } \\
\text { interface }\left[W /\left(m^{2}-k\right)\right]\end{array}$ \\
\hline hSG & $\begin{array}{l}\text { Structure-side heat-transfer coefficient for structure-vapor interface } \\
{\left[W /\left(\mathrm{m}^{2}-\mathrm{k}\right)\right]}\end{array}$ \\
\hline$h_{\mathrm{fm}}$ & Specific heat of fusion enthalpy of component $\mathrm{m} \mathrm{J} / \mathrm{kg}]$ \\
\hline$h_{f, M}$ & Specific heat of fusion enthalpy of material $\mathrm{M}[\mathrm{J} / \mathrm{kg}]$ \\
\hline$h_{I g, M}$ & Heat of vaporization enthalpy $[\mathrm{J} / \mathrm{kg}]$ \\
\hline $\mathrm{i}_{\mathrm{m}}$ & Specific enthalpy of component $\mathrm{m}[\mathrm{J} / \mathrm{kg}]$ \\
\hline $\mathrm{i}_{\mathrm{Con}, \mathrm{M}}$ & Condensation liquid specific enthalpy $[\mathrm{J} / \mathrm{kg}]$ \\
\hline iVap,M & Saturated vapor specific enthalpy $[\mathrm{J} / \mathrm{kg}]$ \\
\hline $\mathrm{k}_{\mathbf{r}}$ & Energy-component thermal conductivity $[\mathrm{W} /(\mathrm{m}-\mathrm{K})]$ \\
\hline $\mathrm{m}$ & Density-component subscript ( 1 through 7$)$ \\
\hline $\mathbf{M}$ & Material subscript (1 through 2 or 3 ) \\
\hline $\mathrm{p}$ & Pressure $\left[\mathrm{N} / \mathrm{m}^{2}\right]$ \\
\hline PG & Total gas pressure $\left[\mathrm{N} / \mathrm{m}^{2}\right]$ \\
\hline PGM & Partial pressure of vapor material component $M\left[N / \mathrm{m}^{2}\right]$ \\
\hline $\mathrm{PCrt}, \mathrm{M}$ & Critical pressure of material $\mathrm{M}\left[\mathrm{N} / \mathrm{m}^{2}\right]$ \\
\hline PSat,M & Saturation pressure of material $M\left[N / \mathrm{m}^{2}\right]$ \\
\hline PSId,M & Solid EOS pressure of material $\mathrm{M}\left[\mathrm{N} / \mathrm{m}^{2}\right]$ \\
\hline PSpg,M & Vapor spinodal limit pressure of material $M\left[N / \mathrm{m}^{2}\right]$ \\
\hline PSpl,M & Liquid spinodal limit pressure of material $M\left[N / \mathrm{m}^{2}\right]$ \\
\hline PSub,M & Sublimation pressure of material $\mathrm{M}\left[\mathrm{N} / \mathrm{m}^{2}\right]$ \\
\hline PVan,M & Van-der-Waals type pressure of material $\mathrm{M}\left[\mathrm{N} / \mathrm{m}^{2}\right]$ \\
\hline r & Energy-component subscript \\
\hline $\mathbf{R}^{\mathbf{o}}$ & Universal gas constant $[\mathrm{J} /(\mathrm{kg}-\mathrm{K})]$ \\
\hline $\mathbf{R}_{\mathbf{M}}$ & Material gas constant $[\mathrm{J} /(\mathrm{kg}-\mathrm{K})]$ \\
\hline $\mathrm{T}$ & Temperature $[\mathbf{K}]$ \\
\hline $\mathrm{T}_{\mathrm{Crt}, \mathrm{M}}$ & Critical temperature of material $\mathrm{M}[\mathrm{K}]$ \\
\hline $\mathrm{T}_{\mathrm{G}}$ & Temperature of gas mixture (vapor temperature) [K] \\
\hline $\mathbf{T}_{\text {Liq, }} \mathbf{M}$ & Input material liquidus temperature $[\mathrm{K}]$ \\
\hline $\mathrm{T}_{\mathrm{Lm}}$ & Temperature of liquid component $m[K]$ \\
\hline $\mathbf{T}_{\mathbf{P}}$ & Temperature of particle component $[\mathrm{K}]$ \\
\hline$T_{S}$ & Temperature of structure component $[\mathrm{K}]$ \\
\hline $\mathrm{T}_{\text {Sat, }} \mathrm{M}$ & Saturation temperature $[\mathrm{K}]$ \\
\hline TSol,M & Input material solidus temperature $[\mathrm{K}]$ \\
\hline $\mathrm{r}_{\mathrm{GLC}, \mathrm{M}}$ & Temperature parameter in surface tension relation $[\mathrm{K}]$ \\
\hline
\end{tabular}


TSLLIR Temperature parameter in surface tension relation [K]

\section{Greek Symbols}

$\alpha_{0} \quad$ Minimum input vapor volume fraction

$\tilde{\alpha} \quad$ Minimum effective vapor volume fraction $\left[\tilde{\alpha}=\alpha_{0}\left(1-\alpha_{s}\right)\right]$

$\alpha_{\mathrm{ge}} \quad$ Effective gas volume fraction for two-phase flow $\left[\alpha_{\mathrm{ge}}=\max \left(\alpha_{\mathrm{geff}}, \tilde{\alpha}\right)\right]$

$\alpha_{\text {Geff }} \quad$ Effective gas volume fraction

$\varepsilon / \kappa_{M} \quad$ Lenard-Jones parameters [K]

$\mu_{\mathrm{r}} \quad$ Energy-component dynamic viscosities $[\mathrm{kg} /(\mathrm{m}-\mathrm{s})]$

$\rho_{\mathrm{m}} \quad$ Density of component $\mathrm{m}$ (microscopic density) $\left[\mathrm{kg} / \mathrm{m}^{3}\right]$

$\rho$ Con, $M$ Condensated liquid density of material $M\left[\mathrm{~kg} / \mathrm{m}^{3}\right]$

$\rho C r t, M \quad C r i t i c a l$ density of material $M\left[\mathrm{~kg} / \mathrm{m}^{3}\right]$

$\rho L, M \quad$ Microdensity of liquid material $M\left[\mathrm{~kg} / \mathrm{m}^{3}\right]$

$\rho S, M \quad$ Microdensity of structure material $\mathrm{M}\left[\mathrm{kg} / \mathrm{m}^{3}\right]$

$\rho S p g, M \quad$ Vapor spinodal limit density of material $M\left[\mathrm{~kg} / \mathrm{m}^{3}\right]$

PSus, $M \quad$ Solid density of material $\mathbf{M}$ at sublimation $\left[\mathrm{kg} / \mathrm{m}^{3}\right]$

$\rho$ Vap, $M \quad$ Saturated vapor density of material $M\left[\mathrm{~kg} / \mathrm{m}^{3}\right]$

$\bar{\rho}_{\mathrm{m}} \quad$ Macrodensity $(\alpha \times \rho)$ of component $\mathrm{m}$ (smear-density) $\left[\mathrm{kg} / \mathrm{m}^{3}\right]$

$\sigma_{\mathrm{M}} \quad$ Material molecular diameter $\left[10^{-10} \mathrm{~m}\right]$

$\sigma_{\mathrm{LN}, \mathrm{M}} \quad$ Surface tension parameter $\left[\mathrm{kg} / \mathrm{s}^{2}\right]$

$\sigma_{L F L, M} \quad$ Surface tension parameter $\left[\mathrm{kg} / \mathrm{s}^{2} / \mathrm{K}\right]$

$\sigma_{L F E, M}$ Surface tension parameter $\left[\mathrm{kg} / \mathrm{s}^{2}\right]$

$\sigma_{\mathrm{LEX}, \mathrm{M}}$ Surface tension parameter

$\sigma_{\text {LLIN }}$ Surface tension parameter $\left[\mathrm{kg} / \mathrm{s}^{2}\right]$

$\sigma_{\text {LLIF }}$ Surface tension parameter $\left[\mathrm{kg} / \mathrm{s}^{2} / \mathrm{K}^{2}\right]$ 


\begin{abstract}
This volume of the Advanced Fluid-Dynamics Model (AFDM) documents the modeling of the equation of state (EOS) in the code. We present an overview of the basic concepts underlying the thermodynamics modeling and resulting EOS, which is a set of relations between the thermodynamic properties of materials.

The AFDM code allows for multiphase-multimaterial systems, which we explore in three phase models: two-material solid, two-material liquid, and threematerial vapor. We describe and compare two ways of specifying the EOS of a materials: (1) as simplified analytic expressions, or (2) as tables that precisely describe the properties of materials and their interactions for mechanical equilibrium. Either of the two EOS models implemented in AFDM can be selected by specifying the option when preprocessing the source code for compilation.

Last, we determine thermophysical properties such as surface tension, thermal conductivities, and viscosities in the model for the intracell exchanges of AFDM. Specific notations, routines, EOS data, plots, test results, and corrections to the code are available in the appendices.
\end{abstract}




\title{
AFDM: AN ADVANCED FLUID-DYNAMICS MODEL
}

\section{Vol. VI: EOS-AFDM Interface}

\author{
Compiled by \\ G. Henneges and S. Kleinheins
}

Contributors

W. R. Bohl, E. A. Fischer, L. Goutagny, P. J. Maudlin, H. Ninokata, F. R. Parker, and D. Wilhelm

\section{INTRODUCTION}

\section{A. Thermodynamic vs Mechanical Equilibrium}

This volume of the Advanced Fluid-Dynamics Model (AFDM) documentation deals with the modeling of the equation of state (EOS) in the code. The EOS is involved wherever thermodynamics is addressed. This is the case in all parts of the code, particularly in Step 1. To start with, an overview of the basic concepts underlying the thermodynamics modeling is presented.

The EOS is a set of relations between the thermodynamic properties of materials. The materials most likely to be used in the code's applications are plutoniumuranium-mixed oxide fuel, stainless steel, and sodium. The materials in the code are assumed to be inert substances and immiscible with respect to each other (see Sec. I.B), such that the definition of a unique EOS for each material will suffice.

As is well known from textbooks, the EOS of a material is derived under the assumption of thermodynamic equilibrium. This implies thermal equilibrium ( $\mathrm{T}=$ const.), phase equilibrium (the chemical potential is the same for different phases), and mechanical equilibrium ( $p=$ const.). Then, apart from the temperature $T$ and the pressure $p_{0}$ the thermodynamic properties entering the EOS are the densities $\rho$, specific internal energies e, specific enthalpies $i$, etc., of the phases, and there are unique relations between these properties. In the AFDM code, $\rho$ and $T$ generally are the independent properties. Of special interest are those values of the properties 
where two phases are in equilibrium (vaporization, condensation, sublimation, melting, and freezing). Also derived from the EOS are the derivatives of the dependent properties with respect to the independent properties (such as specific heat capacities, compressibilities, etc.).

The thermodynamic systems under consideration in the code are the mesh cells of the discretized fluid dynamical domain. Except for the initialization phase, the systems resulting at any time from the solution of the fluid dynamics equations are usually not in thermodynamic equilibrium, nor are they presumed to pass into that state. Instead, thermodynamic equilibrium holds only within each component (see Sec. I.B), but the system as a whole is assumed to pass into mechanical equilibrium for the given constant masses (or macrodensities $\bar{\rho}$ ) and temperature $\mathrm{T}$ of the components. This state is expected to be established very rapidly (the equilibration process is not modeled in the code), such that the components virtually are in mechanical equilibrium at all times. The properties resulting from this "instantaneous" process are the pressure $p$, the densities $\rho$ (and thus the volume fractions $\alpha=\bar{\rho} / \rho$ ), specific internal energies e, and specific enthalpies i of the components. Note that for this concept, the equilibrium EOS of the materials must be extended to provide for subcooling and overheating of the phase. Care must be taken not to exceed the physically sensible limits.

The volume fraction adjustments to achieve mechanical equilibrium assume no heat and mass transfer take place. Rather, the differences in temperature and chemical potential between the components in the mechanically equilibrated system (i.e., the deviation from thermodynamic equilibrium) provide the rates that drive the heat- and mass-transfer processes in the cell (such as phase transition and heat conduction). These rates enter the source terms of the fluid dynamics partial differential equations and are adjusted by an iteration involving the EOS. The heat- and mass-transfer models constitute a major fraction of the code. They are, however, not the subject of this volume; see Vols. II, III, and IV of the AFDM documentation for the treatment of these features.

\section{B. Multiphase-Multimaterial Systems}

The AFDM code allows for systems consisting of three materials (usually fuel, steel, and sodium or fission gas) in three phase states. However, not all materials can exist in all phase states or can take part in all possible phase transitions. There are three phase models: two-material solid, two-material liquid, and three-material vapor. One of the solid phase materials (the "structure component") is assumed not 
to take part in any phase-transition; also it is assumed incompressible." The other solid phase material (the "particle component") can melt into the liquid phase or can freeze out from the liquid phase (but does not evaporate). The liquid phases of both materials can evaporate or condensate into/from the vapor phase. The third material is assumed noncondensible; that is, it exists in the vapor phase only.

In the liquid phase, the materials are assumed immiscible, that is, each material keeps its properties irrespective of the presence of the other material. The vapor phase mixture is assumed to obey Dalton's law; that is, the total vapor pressure is the sum of the vapor material partial pressures. The vapor mixture specific internal energy is the mass-weighted average of the vapor material internal energies.

Together there are seven "microscopically" traceable components ("density components") in the code that collapse into five "macroscopically" distinguishable components ("energy-components") because of the homogeneous vapor mixture (see App. A, Table I).

\section{Simplified Analytic EOS vs Precise Tabular EOS (TEOS)}

There are two ways of specifying the EOS of a material: as analytic expressions or as tables of the dependent properties over the independent properties. Both ways are implemented in the AFDM code, the first in the form of a simplified analytic EOS, the second based upon the SESAME EOS Data Library and other data.

The model of the simplified analytic EOS not only assumes simple EOS relations (ideal gas equation, constant liquid compressible, temperature-independent solid and liquid density, constant solid and liquid specific heat capacities, etc.), but also implies simplified assumptions for the mechanical equilibrium. These include an incompressible liquid in two-phase systems and an approximate explicit equation for the single-phase system pressure. To provide an acceptable transition between the two-phase and single-phase states under these simplifications, the " $\alpha_{0}$ concept" (see Sec. II.A) has been introduced into this model. All these assumptions allow straightforward determination of the mechanical equilibrium pressure and the derivatives of the properties.

The TEOS, on the other hand, precisely describes the properties of the materials and their interaction for mechanical equilibrium. Thus, an iterative algorithm

\footnotetext{
"Because the structure component has a constant macroscopic density, it need not be physically and chemically related to the corresponding liquid component. The default input provided in the code with steel for both components can be overridden by values for other solid substances (also see footnote in Sec. II.B:I).
} 
to determine the mechanical equilibrium is required. In this model, the derivatives of the properties are defined under the assumption of mechanical equilibrium and thus are involved expressions. Because of the decoupling of heat and mass transfer from convection in the AFDM algorithm, the $\alpha_{0}$ concept has been maintained to provide a cushion when small vapor volume fractions exist. Note that a more sophisticated analytic EOS would have required the same treatment.

A comparison of the two methods implemented in the code shows that the first yields simple coding, fast running times, but less-accurate results, whereas the second is the opposite in each respect. Further contributing to the higher effort with the TEOS is the table search and interpolation. The spinodal limits of the real gas and real liquid EOS require special treatment that is not necessary for the simplified analytical EOS. Finally, the inversion of the thermal EOS (that is, give $\bar{\rho}$ and e, find $\rho$ and $T$ ) is more complicated for the tabular than for the simplified analytic EOS.

\section{EOS Implementation in the Code}

Either of the two EOS models implemented in the AFDM code can be selected by specifying the option at the time of preprocessing the source code for compilation. The code is designed such that all thermodynamic properties and derivatives thereof are obtained from calls of so-called EOS-interface routines. With the exception of the mechanical equilibrium routine, there are two versions of each of the 13 EOS interface routines (see App. B). The specification of the preprocessor option for either the analytical or the TEOS inserts the appropriate set of interface routines into the compilable code. All interface routines and their subroutines are vectorized to save computing time.

Whereas the parameters for the simplified analytical EOS are supplied to the code by default or explicit input, the TEOS requires the attachment of a file in the SESAME EOS Data Library format.

\section{E. Thermophysical Properties}

For the intracell exchange model, quantities such as surface tensions, thermal conductivities, and viscosities of the components are needed. Because they are related to and derived from the thermodynamic properties, the models for their determination are described in this volume. 


\section{THE SIMPLIFIED ANALYTIC EQUATION OF STATE (SAEOS)}

This chapter gives a description of the formulas used for the SAEOS option in AFDM. There are five parts in the presentation. First, a description of the simplifications and assumptions; second, the derivation of the EOS functions (the AFDM algorithm requires the internal energies and enthalpies, microscopic densities, cell pressure, and saturation temperatures corresponding to the vapor partial pressures); third, the derivatives used in the pressure iteration; fourth, the algorithm for inverting the equation of state; and fifth, expressions for the derivatives used in step 1 .

\section{A. Basic Assumptions}

The basis of the SAEOS algorithm is to divide the domain of the effective vapor volume fraction, $\alpha$, defined by

$$
\alpha=\alpha_{\text {Geff }} /\left(1-\alpha_{\mathrm{s}}\right)
$$

into two regions,

$$
\begin{aligned}
& \alpha \geq \alpha_{0} \text { being a two-phase region, and } \\
& \alpha<\alpha_{0} \text { being a single-phase region, }
\end{aligned}
$$

where

$$
\begin{aligned}
\alpha_{\text {Geff }}= & 1-\alpha_{S}-\left(1-\alpha_{0}\right) \sum_{m=2}^{4} \bar{\rho}_{m} /\left(\rho_{m}\right), \\
\alpha_{S}= & \bar{\rho}_{S} / \rho_{S} \text { the structure volume fraction, } \\
\bar{\rho}_{\mathrm{m}}= & \text { the smear (macroscopic) density for component } \mathrm{m}, \\
\rho_{\mathrm{m}}= & \text { the thermodynamic (microscopic) density for component } \mathrm{m} \text {, and the } \\
& \text { summation over components } 2 \text { to } 4 \text { comprises the particles and both } \\
& \text { liquids. }
\end{aligned}
$$

The quantity $\alpha_{0}$ is an input constant, nominally about 0.01 to 0.05 . In the two-phase region, the vapor is assumed to be the only compressible species, and the liquids simply occupy space. In the single-phase region, the vapor is assumed to 
occupy the volume $\tilde{\alpha}=\alpha_{\mathrm{o}}\left(1-\alpha_{\mathrm{s}}\right)$, and liquids provide additional compressibility. The reason for the inclusion of $\alpha_{0}$ in the definition of $\alpha_{G e f f}$ is to have the twophase-to-single-phase transition take place when the liquid occupies the entire volume available for fluid flow. The principal reason for inclusion of $\alpha_{0}$ in the AFDM EOS comes from the time factorization algorithm. In step 3 , all vapor species are temporarily treated as noncondensible, and $\alpha_{0}$ provides a cushion in treating the single-phase-to-two-phase transition. Further, in the SAEOS, where vapor provides the only compressibility for a two-phase cell, large and rapidly changing derivatives for small values of $\alpha$ will lead to unacceptably slow convergence of the pressure iteration without $\alpha_{o}$.

Other assumptions used in the SAEOS are that the structure, particle, and liquid microscopic densities are input coristants, and their internal energies are assumed to depend only on temperature with constant heat capacities. For fuel, the difference between the solidus and liquidus energies is given by an input heat of fusion. Pressures in single-phase liquid cells are obtained using constant soric velocities for particles and liquids. The enthalpy of vaporization is constant, leading to a vapor-pressure relationship based on integrating the Clausius-Clapeyron equation. The vapor heat capacities representing the departure from the saturation curves are constants, and input material-dependent gas constants are used to relate partial pressures, densities, and a mixture vapor temperature.

To further organize the presentation, the idea of material components, $M$, is introduced. These components are

$$
\begin{aligned}
& M=1 \text { = fuel, } \\
& M=2 \text { = steel (if structure) or coolant (if liquid or vapor), and } \\
& M=3 \text { = noncondensible gas. }
\end{aligned}
$$

These material component subscripts are supplemented by $\mathrm{m}$ (density) and $\mathbf{r}$ (energy) subscripts as appropriate. Also, subscript $\mathrm{S}$ (representing structure, or energy and density component 1 ), subscript $P$ (representing particles, or energy and density component 2), subscript L (signifying liquids, such that energy and density components 3 and 4 are represented by L1 and L2), and subscript $G$ (representing vapor, or energy component 5) are used for clarity. (See Apps. A and D for notation and definitions.) 


\section{B. Functions Provided by the SAEOS}

The SAEOS formalism is built by first defining the material internal energies and enthalpies. The pressure for the vapor species is then obtained from the ideal gas law. In single-phase cells, a liquid pressure contribution is defined assuming each liquid/particle component is equally compressed. Closure is obtained for the SAEOS by integrating the Clausius-Clapeyron equation.

1. Internal Energies and Enthalpies. The structure- and particle-specific internal energies are given by

$$
e_{r}=c_{v S, M} T_{r}, r=1,2
$$

where

$$
\begin{aligned}
\mathrm{c}_{\mathrm{vS}, \mathrm{M}} & =\text { the solid material heat capacity }[\mathrm{J} / \mathrm{kg}-\mathrm{K}]^{*}, \\
\mathrm{~T}_{\mathrm{r}} & =\text { the absolute temperature in degrees } \mathrm{K}, \\
\mathrm{M} & =2 \text { (steel) corresponds to } \mathrm{r}=1 \text { (structure), and } \\
\mathrm{M} & =1 \text { (fuel) corresponds to } \mathrm{r}=2 \text { (particles). }
\end{aligned}
$$

The liquid-specific internal energies are given by

$$
e_{r}=c_{v S, M} T_{S o l, M}+h_{f, M}+c_{v L, M}\left(T_{r}-T_{L i q, M}\right), r=3,4,
$$

where

$$
\begin{aligned}
c_{\mathrm{VS}, M} & =\text { the solid material heat capacity }[\mathrm{J} / \mathrm{kg}-\mathrm{K}] \\
\mathrm{c}_{\mathrm{VL}, \mathrm{M}} & =\text { the liquid material heat capacity }[\mathrm{J} / \mathrm{kg}-\mathrm{K}] \\
\mathrm{T}_{\mathrm{r}} & =\text { the liquid temperature in degrees } \mathrm{K}, \\
\mathrm{h}_{\mathrm{f}, \mathrm{M}} & =\text { the heat of fusion }[\mathrm{J} / \mathrm{kg}] \\
\mathrm{T}_{\mathrm{Sol}, \mathrm{M}} & =\text { the (input) material solidus temperature, } \\
\mathrm{T}_{\mathrm{Liq}, \mathrm{M}} & =\text { the (input) material liquidus temperature, } \\
\mathrm{M} & =1 \text { corresponds to } \mathrm{r}=3, \text { and } \\
\mathrm{M} & =2 \text { corresponds to } \mathrm{r}=4 .
\end{aligned}
$$

${ }^{*} \mathrm{CS}, 2$ is used both for the structure and for material liquid/vapor internal energies. If structure represents a substance different from that of liquid/vapor 2 , an artificial $h_{f, 2}$ can be used to adjust the normalization of the liquidus energy. 
For the melting and freezing model, the solidus and liquidus energies are required. Examination of Eq. (2.3) suggests that reasonable SAEOS expressions are

$$
\begin{aligned}
& \mathrm{e}_{\mathrm{Sol}, \mathrm{M}}=\mathrm{C}_{\mathrm{v} S, \mathrm{M}^{\mathrm{T}} \mathrm{Tol}, \mathrm{M}, \text { and }} \\
& e_{\text {Liq, }, M}=c_{v S, M} T_{S o l, M}+h_{f, M}, M=1,2 .
\end{aligned}
$$

The liquid condensate energies are given by

$$
e_{C o n, M}=e_{L i q, M}+e_{v L, M}\left(T_{S a t, M}-T_{L i q, M}\right), M=1,2
$$

where

$$
\mathrm{T}_{\mathrm{Sat}, \mathrm{M}}=\text { the saturation temperature for material } \mathrm{M} \text {. }
$$

Saturation temperatures are defined by Eq. (2.23), based on the vapor partial pressures. The liquid condensate enthalpy is given by

$$
\mathrm{i}_{\text {Con }, M}=\mathrm{eCon} \mathrm{M}+\mathrm{p}_{\mathrm{GM}} / \rho_{\mathrm{r}}, \mathrm{r}=3,4 \text {, }
$$

where

$$
\begin{aligned}
P_{G M} & =\text { the vapor partial pressure for material } M, \\
\rho_{r} & =\text { the microscopic density for component } r, \text { and } \\
M & =1 \text { for } r=3, \text { and } \\
M & =2 \text { for } r=4 .
\end{aligned}
$$

In the SAEOS, the difference between the condensate enthalpy and condensate energy should be small; however, the condensate enthalpy is used in the masstransfer models, so it is computed here. The vapor saturation energy for both fuel and coolant is given by

$$
e_{V a p, M}=e_{C o n, M}+h_{g, M}-p_{G M}\left(1 / \rho_{V a p, M}-1 / \rho_{r}\right), r=3,4 \text {, }
$$


where

$$
\begin{aligned}
h_{l g, M} & =\text { the enthalpy of vaporization }[\mathrm{J} / \mathrm{kg}] \\
\rho V_{a p, M} & =\text { a vapor component microscopic density at saturation, } \\
M & =1 \text { for } r=3, \text { and } \\
M & =2 \text { for } r=4 .
\end{aligned}
$$

The enthalpy of vaporization is obtained from the input, as discussed following Eq. (2.22). A vapor's partial pressure is given below in Eq. (2.19). The vapor component microscopic density at saturation is obtained in the SAEOS by using Eqs. (2.19) and (2.22). Then, to evaluate the work term in Eq. (2.8), the volume of the liquid is neglected such that the final approximation to eVap, $M$ is given in Eq. (2.25). The specific internal energy for vapor material components 1 and 2 is given by the deviation from saturation conditions, or

$$
\text { eG,M }=e_{\text {Vap }, M}+c_{v G, M}\left(T_{G}-T_{\text {Sat }} M\right), M=1 \text { and } 2,
$$

where

$$
\begin{aligned}
c_{v G, M} & =\text { the vapor heat capacity }[J / \mathrm{kg}-\mathrm{K}], \text { and } \\
T_{G} & =\text { the vapor temperature. }
\end{aligned}
$$

For noncondensible gas, the specific internal energy is given by the increase over the critical energy as

$$
\mathbf{e}_{\mathrm{G}, 3}=\mathbf{e}_{\mathrm{Crt}, 3}+\mathrm{c}_{\mathrm{VG}, 3}\left(\mathrm{~T}_{\mathrm{G}}-\mathrm{T}_{\mathrm{Crt}, 3}\right)
$$

where

$\mathrm{e}_{\mathrm{Crt}, 3}=$ an input critical energy $[\mathrm{J} / \mathrm{kg}]$, and

$\mathbf{T}_{\mathrm{Crt}, 3}=$ an input critical temperature.

The cell-averaged vapor specific internal energy is given by a mass average as

$$
e_{G}=\sum_{M=1}^{3} x_{M} e_{G, M},
$$


where

$$
x_{\mathrm{M}}=\bar{\rho}_{\mathrm{GM}} /\left(\bar{\rho}_{\mathrm{G} 1}+\bar{\rho}_{\mathrm{G} 2}+\bar{\rho}_{\mathrm{G} 3}\right) .
$$

The vapor enthalpies corresponding to Eqs. (2.8) through (2.11) are given by

$$
\begin{aligned}
& i_{V a p, M}=i_{C o n, M}+h_{g, M}, M=1,2, \\
& i_{G, M}=e_{G, M}+p_{G M} / P_{G M}, M=1,2, \text { and } 3, \text { and } \\
& i_{G}=\sum_{M=1}^{3} x_{M G, M} i_{G},
\end{aligned}
$$

where $\rho_{G M}$ is the vapor component microscopic density.

2. Microscopic Densities and Pressures. The vapor microscopic densities are based on dividing the smear densities calculated from the conservation equations by the total available vapor volume, or

$$
\rho_{\mathrm{GM}}=\bar{\rho}_{\mathrm{GM}} /\left[\max \left(\tilde{\alpha}, \alpha_{\mathrm{Geff}}\right)\right], M=1,2 \text {, and } 3 \text {, }
$$

shere

$\tilde{\alpha}$ and $\alpha_{\text {Geff }}$ were defined following Eq. (2.1).

The structure, particle, and liquid microscopic densities are assumed to be input by the users.

For two-phase cells, the pressure is evaluated as the sum of the partial pressures. This means

$$
p=p_{G}=\sum_{M=1}^{3} p_{G M}=\sum_{M=1}^{3} \rho_{G M} R_{M} T_{G} \text {, }
$$

where

$$
R_{M}=\text { an input material-dependent gas constant. }
$$


For single-phase cells, an additional contribution is added to the total pressure from compressed liquid. The derivation for the pressure in single-phase cells follows that of a personal communication. 1 Each liquid density component is presumed to see the same pressure, or

$$
p=p_{\mathrm{ln}}=p_{G}+c_{m}^{2} \delta p_{m}
$$

where

$$
\begin{aligned}
\mathrm{Pm}_{\mathrm{m}}= & \text { the pressure in liquid density component } \mathrm{m}, \\
\mathrm{P}_{\mathrm{G}}= & \text { the pressure at the two-phase/single-phase transition, } \\
c_{m}= & \text { the sonic velocity for liquid density component } \mathrm{m}_{\mathrm{i}} \text {, and } \\
\delta \rho_{\mathrm{m}}= & \text { the change in density from the state at the single-phase/two-phase } \\
& \text { transition to the current single-phase liquid state. }
\end{aligned}
$$

The change in density is obtained by assuming that the calculated liquid density determined from the conservation equation, or $\bar{\rho}_{\mathrm{m}^{\prime}}$ must fit into a volume given by $\alpha_{m} \cdot \alpha_{m o}$ is the liquid volume fraction for component $m$ at the single-phase-to-twophase transition. This means that

$$
p=p_{G}+c_{m}^{2}\left[\bar{\rho}_{m} / \alpha_{m}-\rho_{m o}\right]
$$

where

$$
\rho_{\mathrm{mo}}=\text { the microscopic density at the single-phase/two-phase transition. }
$$

Eq. (2.15) is multiplied by $\left(\alpha_{m} / c_{m}^{2} \rho_{m o}\right)$ and summed over $m$ to obtain

$$
\left(p-p_{G}\right) \sum_{m=2}^{4} \frac{\alpha_{m}}{c_{m}^{2} \rho_{m o}}=\sum_{m=2}^{4}\left(\frac{\bar{\rho}_{m}}{\rho_{m o}}-\alpha_{m}\right) \text {. }
$$

Eq. (2.16) is simplified using the definitions

$$
\alpha_{m o}=\bar{\rho}_{m} / \rho_{m o}, \text { the liquid volume fraction of component } m \text {, }
$$




$$
\begin{aligned}
\alpha_{\mathrm{L}} & =\sum_{\mathrm{m}} \alpha_{\mathrm{mo}}, \text { the total liquid field volume fraction, and } \\
\sum_{\mathrm{m}} \alpha_{\mathrm{mo}} & =1-\alpha_{S}, \text { the liquid volume fraction at the single-phase state. }
\end{aligned}
$$

These definitions give

$$
\mathrm{p}=\mathrm{p}_{\mathrm{G}}+\left(\alpha_{\mathrm{L}}+\alpha_{\mathrm{s}}-1\right) / \sum_{\mathrm{m}=2}^{4} \frac{\alpha_{\mathrm{m}}}{\mathrm{c}_{\mathrm{m}}^{2} \rho_{\mathrm{mo}}}
$$

The final expression is obtained by means of the simplifying approximation $\alpha_{m} \sim \alpha_{m o}$. This is not exact because $\alpha_{m}$ depends on pressure. It does avoid an iteration and is simpler than other possibilities. In any case, assuming that $c_{m}$ and $\rho_{m}$ depend on cell conditions such as temperature and are not input constants, they should be considered if a more complex expression is desired. Using this approximation, the single-phase cell pressure is

$$
\mathrm{p}=\mathrm{p}_{\mathrm{G}}+\frac{1}{\sum_{\mathrm{m}=2}^{4} \frac{\bar{\rho}_{\mathrm{m}}}{\mathrm{c}_{\mathrm{m}}^{2} \rho_{\mathrm{mo}}^{2}}}\left[\alpha_{\mathrm{L}}+\alpha_{\mathrm{S}}-1\right] .
$$

The pressure in single-phase cells is consequently independent of the liquid temperature when the SAEOS is used, except indirectly as affecting $\mathrm{P}_{\mathrm{G}}$ in the $\tilde{\alpha}$ volume through the vaporization-condensation model.

3. Saturation Temperatures. To obtain an expression for $T_{S a t, M}$, we start with the Clapeyron equation, which can be written as

$$
\frac{\mathrm{dP}_{\mathrm{GM}}}{\mathrm{dT}_{\mathrm{Sat}, M}}=\frac{\mathrm{h}_{\mathrm{lg}, \mathrm{M}}}{\mathrm{T}_{\mathrm{Sat}, M,}\left(1 / \mathrm{P}_{\mathrm{Vap}, M}-1 / \rho_{\mathrm{r}}\right)},
$$

where

$$
\begin{aligned}
& M=1 \text { for } r=3, \text { and } \\
& M=2 \text { for } r=4 .
\end{aligned}
$$


By making the Clausius approximations of assuming that the vapor obeys the ideal gas law, or

$$
p_{G M}=\rho_{G M} R_{M} T_{G}=\rho_{V a p, M} R_{M} T_{S a t, M}, M=1,2,
$$

and neglecting the volume of the liquid, Eq. (2.18) becomes the Clausius-Clapeyron equation

$$
\frac{\mathrm{dp}_{\mathrm{GM}}}{\mathrm{dT}_{\text {Sat }, \mathrm{M}}}=\frac{\mathrm{h}_{\mathrm{lg}, \mathrm{M}} \mathrm{P}_{\mathrm{GM}}}{\mathrm{R}_{\mathrm{M}} \mathrm{T}_{\text {Sat, } \mathrm{M}}^{2}}, \mathrm{M}=1,2
$$

Eq. (2.20) may be rearranged and integrated as

$$
\text { in } \mathrm{p}_{\mathrm{GM}}=-\frac{\mathrm{h}_{\mathrm{lg}, \mathrm{M}}}{\mathrm{R}_{\mathrm{M}} \mathrm{T}_{\mathrm{Sat}, \mathrm{M}}}+\ln \mathrm{p}_{\mathrm{M}}^{*}, \mathrm{M}=1,2
$$

where

$$
\ln \mathrm{P}_{M}^{*}=\text { the constant of integration. }
$$

Equation (2.21) can be written as

$$
\mathrm{P}_{\mathrm{GM}}=\mathrm{P}_{\mathrm{M}}^{*} \exp \left(-\mathrm{T}_{\mathrm{M}}^{*} / \mathrm{T}_{\mathrm{Sat}, \mathrm{M}}\right), \mathrm{M}=1,2 \text {, }
$$

where

$$
\mathrm{T}_{M}^{*}=\mathrm{h}_{\mathrm{g}, \mathrm{M}} / \mathrm{R}_{\mathrm{M}}
$$

In the SAEOS, $p_{M}^{*}, R_{M}$, and $T_{M}^{*}$ are input, making $h_{l g}, M$ a calculated quantity. From Eq. (2.22), $T_{S a t, M}$ may be determined as

$$
\mathrm{T}_{\mathrm{Sat}, \mathrm{M}}=-\mathrm{T}_{\mathrm{M}}^{*} / \ln \left(\mathrm{p}_{\mathrm{GM}} / \mathrm{p}_{\mathrm{M}}^{*}\right), \mathrm{M}=1,2
$$


By making the assumption of Eq. (2.20), (assuming the vapor obeys the ideal gas law, and neglecting the volume of the liquid), the work term in Eq. (2.8) can be evaluated as

$$
P_{G M}\left(1 / p_{\text {Vap }, M}-1 / p_{r}\right)=\frac{T_{\text {Sat }, M}}{T_{M}^{*}} h_{l g, M}=R_{M} T_{S a t, M}
$$

where

$$
\begin{aligned}
& M=1, \text { for } r=3, \text { and } \\
& M=2 \text { for } r=4 .
\end{aligned}
$$

This allows Eq. (2.8) to be approximated as

$$
\mathrm{e}_{\mathrm{Vap}, M}=\mathrm{e}_{\mathrm{Con}, \mathrm{M}}+\mathrm{h}_{\mathrm{lg}, \mathrm{M}}\left(1-\frac{\mathrm{T}_{\text {Sat, } M}}{\mathrm{~T}_{M}^{*}}\right)=\mathrm{e}_{\mathrm{Con}, \mathrm{M}}+\mathrm{R}_{\mathrm{M}}\left(\mathrm{T}_{\mathrm{M}}^{*}-\mathrm{T}_{\text {Sat }, M}\right) \text {, }
$$

where

$$
\mathbf{M}=1 \text { and } 2 .
$$

This completes the expressions for the SAEOS functions. At this point, some observations appear appropriate on the assumptions made. A better expression for the heat of vaporization is

$$
h_{l g, M}=\left(h_{l g, M}\right)+\int_{0}^{T}\left(c_{p G, M}-c_{p L, M}\right) d T
$$

where

$\left(h_{\mathrm{lg}}, \mathrm{M}\right)_{\mathrm{O}}=$ the hypothetical heat of vaporization at absolute zero, $\mathrm{C}_{\mathrm{PG}, \mathrm{M}}=$ the constant pressure heat capacity for the vapor, and $c_{\mathrm{PL}, \mathrm{M}}=$ the constant pressure heat capacity for the liquid. 
In the SAEOS, $c_{p L, M}$ equals $c_{v L, M}$ because the liquid pressure [Eq. (2.17)], is independent of the liquid temperature. The value of $c_{p G, M}$ should be $c_{V G, M}+R_{M}$. If $h_{l g, M}$ is to be constant, $c_{v L, M}$ should equal $c_{V G, M}+R_{M}$. Allowing these variables to be input separately leads the SAEOS to an inconsistent density dependence in the vapor internal energy. In other words, the condensible components do not represent ideal gas behavior, and the vapor temperature must be determined from the vapor internal energy using an iteration. Unfortunately, the options to obtain consistency give rise to additional problems. Allowing the user to input only one heat capacity will not represent the fact that $c_{\mathrm{PL}, \mathrm{M}}$ and $\mathrm{c}_{\mathrm{pG}, \mathrm{M}}$ can be significantly different, particularly for simulating saturated sodium at $1 \mathrm{~atm}$. Using a heat of vaporization that depends linearly on temperature [as inferred from Eq. (2.26), assuming constant heat capacities] gives rise to a three-parameter vapor-pressure expression when integrating the Clausius-Chapeyron equation. A three-parameter equation can better fit the data, but now an iteration is required to determine the saturation temperature as a function of pressure. Also, the decreasing $h_{/ g}, M$ predicted from Eq. (2.26) occurs because the liquid expands as the temperature rises; because the molecules are further apart, less energy is required to separate them in forming the vapor This means that with a variable $h_{/ g, M}$, the SAEOS constant liquid microscopic density is inconsistent and can be expected to lead to difficulties at high-enough temperatures. In other words, a variable $h_{/ g, M}$ would mean that further development would be required to represent a critical point in the SAEOS such that the liquid and vapor densities become equal when $h_{\mathrm{g}, \mathrm{M}}$ goes to zero. It should be clear that the SAEOS, as written, does not predict a critical point. Of course, the critical point obtained by assuming the vapor to be an ideal gas and the heat of vaporization to have a linear temperature dependence would not be realistic. And the availability of a temperature-dependent liquid density would soon lead to the desire for temperature dependence in other quantities, such as the sonic velocities. In any case, the vapor internal energy density dependence in the SAEOS gives no apparent stability problems, and the user can remove it by selecting input such that $c_{V L, M}=c_{V G, M}+R_{M}$. Further, the SAEOS obviously only can be expected to represent data over a limited temperature range. Additional sophistication or use of the SESAME option is likely to be required to obtain reliable quantitative results for most nonisothermal situation. For the default values, see App. E. 


\section{Pressure Iteration Derivatives}

Moving on to the functional derivatives needed for the AFDM algorithm, in the pressure iteration, the derivatives of the pressure and vapor internal energy are required with respect to the momentum densities and the vapor temperature. The liquid internal energies are independent of density and vapor temperature; consequently, their derivatives are not required. The momentum densities are given by

$$
\begin{aligned}
& \bar{\rho}_{q=1}=\bar{\rho}_{p}+\bar{\rho}_{L 1}, \\
& \bar{\rho}_{q=2}=\bar{\rho}_{L 2} \text {, and } \\
& \bar{\rho}_{q=3}=\bar{\rho}_{G 1}+\bar{\rho}_{G 2}+\bar{\rho}_{G 3} .
\end{aligned}
$$

Differentiating with respect to the momentum density means differentiating holding the mass ratios constant. For example, for a function, $f$, we have

$$
\frac{\partial f}{\partial \bar{\rho}_{q=x}}=\sum_{n=1}^{N} \frac{\partial f}{\partial \bar{\rho}_{n}} \frac{\bar{\rho}_{n}}{\bar{\rho}_{q=x}} \text { for } \bar{\rho}_{q=x}=\sum_{n=1}^{N} \bar{\rho}_{n} \text {, }
$$

where $\mathbf{N}$ represents the components in momentum field $\mathrm{x}$.

The derivatives of the pressure in two-phase cells can be obtained using

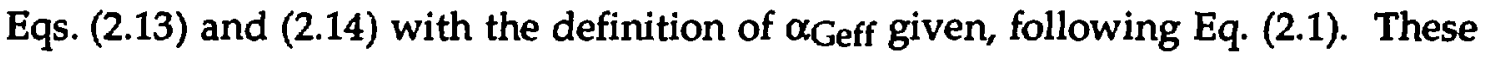
quantities are

$$
\begin{aligned}
& \frac{\partial p}{\partial \bar{\rho}_{q=1}}=\frac{p}{\alpha_{\text {Geff }}} \frac{1-\alpha_{0}}{\bar{\rho}_{p}+\bar{\rho}_{L 1}}\left[\alpha_{p}+\alpha_{L 1}\right], \\
& \frac{\partial p}{\partial \bar{\rho}_{q=2}}=\frac{p}{\alpha_{G e f f}} \frac{1-\alpha_{0}}{\bar{\rho}_{L 2}}, \\
& \frac{\partial p}{\partial \bar{\rho}_{q=3}}=\frac{p}{\bar{\rho}_{G}} \text {, and }
\end{aligned}
$$




$$
\frac{\partial p}{\partial T_{G}}=\frac{p}{T_{G}}
$$

The more complex expression for the pressure in single-phase cells is given schematically by

$$
\mathrm{p}=\mathrm{p}_{\mathrm{G}}+\mathrm{p}_{\mathrm{sf}}
$$

where $p_{s f}$ can be obtained by inspection of Eq. (2.17).

Using this definition for $\mathrm{p}_{\mathrm{sf}}$, the derivatives of the pressure for single-phase cells are

$$
\begin{aligned}
& \frac{\partial p}{\partial \bar{\rho}_{q=1}}=\frac{p_{s f}}{\bar{\rho}_{p}+\bar{\rho}_{L 1}}\left\{\frac{-1}{\sum_{m=2}^{4} \frac{\bar{\rho}_{m}}{c_{m}^{2} \rho_{m}^{2}}}\left[\frac{\bar{\rho}_{p}}{c_{p}^{2} \rho_{p}^{2}}+\frac{\bar{\rho}_{L 1}}{c_{L 1}^{2} \rho_{L 1}^{2}}\right]+\frac{\alpha_{p}+\alpha_{L 1}}{\alpha_{L}+\alpha_{s}-1}\right\}, \\
& \frac{\partial p}{\partial \bar{\rho}_{\mathrm{L} 2}}=p_{\mathrm{sf}}\left\{\frac{-1}{\sum_{\mathrm{m}=2}^{4} \frac{1}{\mathrm{p}_{\mathrm{m}}^{2} \rho_{\mathrm{m}}^{2}}} \frac{1}{\rho_{\mathrm{L} 2}^{2} \mathrm{c}_{\mathrm{L} 2}^{2}}+\frac{1}{\rho_{\mathrm{L} 2}\left(\alpha_{\mathrm{L}}+\alpha_{\mathrm{s}}-1\right)}\right\}, \\
& \frac{\partial p}{\partial \bar{p}_{G}}=\frac{p_{G}}{\bar{\rho}_{G}} \text {, and } \\
& \frac{\partial p}{\partial T_{G}}=\frac{p_{G}}{T_{G}},
\end{aligned}
$$

where

$c_{m}=$ the sonic velocity for energy component $m$,

$m=2$ is the particle component (p),

$\mathrm{m}=3$ is the liquid fuel component.(L1), and

$m=4$ is the liquid coolant component (L2). 
The main difficulty in finding derivatives with respect to the internal energy, $e_{G}$, is the variation in $T_{S a t}, M$. To better express these results, two quantities, $(S C)_{M}$ and $\mathrm{T}_{\mathrm{Sat}, \mathrm{M}^{\prime}}^{\mathrm{P}}$ are defined as

$$
\begin{aligned}
& (S C)_{M}=c_{v L, M}-\frac{h_{l g, M}}{T_{M}^{*}}-c_{v G, M}=c_{v L, M}-R_{M}-c_{v G, M} \text {, and } \\
& \mathrm{T}_{\mathrm{Sat}, \mathrm{M}}^{\mathrm{p}}=\frac{\mathrm{T}_{\text {Sat }, \mathrm{M}}^{2}}{\mathrm{~T}_{\mathrm{M}}^{*}}, \mathrm{M}=1,2 .
\end{aligned}
$$

Then, for two-phase cells, the derivatives of Eq. (2.11) are

$$
\begin{aligned}
& \frac{\partial \mathrm{e}_{\mathrm{G}}}{\partial \bar{\rho}_{\mathrm{q}=1}}=\sum_{M=1}^{2} \mathrm{x}_{M}(\mathrm{SC})_{\mathrm{M}}\left[\mathrm{T}_{\text {Sat,M }}^{\mathrm{p}} \frac{1-\alpha_{\mathrm{o}}}{\alpha_{\mathrm{Geff}}}\left(\frac{\alpha_{\mathrm{p}}+\alpha_{\mathrm{L}}}{\bar{\rho}_{\mathrm{p}}+\bar{\rho}_{\mathrm{L} 1}}\right)\right] \\
& \frac{\partial \mathrm{e}_{\mathrm{G}}}{\partial \bar{\rho}_{\mathrm{q}=2}}=\sum_{\mathrm{M}=1}^{2} \mathrm{x}_{\mathrm{M}}(\mathrm{SC})_{\mathrm{M}}\left[\mathrm{T}_{\mathrm{Sat}, \mathrm{M}}^{\mathrm{M}} \frac{1}{\alpha_{\text {Geff }}} \frac{1-\alpha_{\mathrm{o}}}{\rho_{\mathrm{L} 2}}\right] \text {, } \\
& \frac{\partial \mathrm{e}_{\mathrm{G}}}{\partial \bar{\rho}_{\mathrm{q}=3}}=\sum_{M=1}^{2} \mathrm{x}_{M}(\mathrm{SC})_{M}\left[\mathrm{~T}_{\mathrm{Sat}, \mathrm{M}}^{\mathrm{p}} \frac{1}{\bar{\rho}_{\mathrm{G}}}\right] \text {, and } \\
& \frac{\partial e_{G}}{\partial T_{G}}=\sum_{M=1}^{2} x_{M}\left\{\left(S C_{M}\left[T_{S a t, M}^{p} \frac{1}{T_{G}}\right]+c_{v G, M}\right\}+x_{3} c_{v G, 3} .\right.
\end{aligned}
$$

For single-phase cells, $\mathrm{P}_{\mathrm{GM}}$ and, consequently, $\mathrm{T}_{\mathrm{Sat}} \mathrm{M}$, are independent of the liquid macroscopic density. Consequently, for single-phase cells, where $\alpha_{\text {Geff }}<\alpha_{0}(1-$ as),

$$
\begin{aligned}
& \frac{\partial e_{G}}{\partial \bar{\rho}_{q=1}}=0, \\
& \frac{\partial e_{G}}{\partial \bar{\rho}_{q=2}}=0,
\end{aligned}
$$




$$
\begin{aligned}
& \frac{\partial \mathrm{e}_{G}}{\partial \bar{\rho}_{\mathrm{q}=3}}=\sum_{M=1}^{2} x_{M}\left(S C_{M}\left[T_{S a t, M}^{p} \frac{1}{\bar{\rho}_{G}}\right]\right. \text {, and } \\
& \frac{\partial e_{G}}{\partial T_{G}}=\sum_{M=1}^{2} x_{M}\left\{(S C)_{M}\left[T_{S a t, M}^{p} \frac{1}{T_{G}}\right]+c_{v G, M}\right\}+x_{3} c_{v G, 3} .
\end{aligned}
$$

This concludes the derivatives required from the $3 \mathrm{AEOS}$ for the pressure iteration (step 3 of the AFDM algorithm).

\section{Inversion of the SAEOS}

In both steps 2 and 4, the EOS must be inverted to obtain temperatures from the internal energies. The structure, particle, and liquid temperatures can be obtained in a straightforward fashion from Eqs. (2.2) and (2.3). A Newton-Raphson iteration, used to obtain the vapor temperature, is accomplished by first writing an expression for the error in the vapor internal energy using Eq. (2.11). This expression is labeled as $G$ and is given by

$$
G=\frac{e_{G}-\sum_{M=1}^{2} x_{M}\left[e_{v a p, M}-c_{v G, M} T_{S a t, M}\right]-x_{3}\left[e_{C r t, 3}-c_{v G, 3} T_{C r t, 3}\right]}{\sum_{M=1}^{3} x_{M} c_{v G, M}}-T_{G}=0 .
$$

The iteration starts with a trial $T_{G}^{x}$ and then proceeds by

$$
\mathrm{T}_{G}^{\kappa+1}=\mathrm{T}_{\mathrm{G}}^{\kappa}-\mathrm{G}^{\kappa} /\left[\frac{\partial \mathrm{G}}{\partial \mathrm{T}_{\mathrm{G}}}+\sum_{M=1}^{2} \frac{\partial \mathrm{G}}{\partial \mathrm{T}_{\text {Sat }, M}} \frac{\partial \mathrm{T}_{\mathrm{Sat}, \mathrm{M}}}{\partial \mathrm{T}_{\mathrm{G}}}\right] \text {, }
$$

where

$$
\frac{\partial G}{\partial T_{G}}=-1
$$




$$
\begin{aligned}
& \frac{\partial G}{\partial T_{S a t, M}}=-x_{M} \frac{(S C)_{M}}{\sum_{M=1}^{3} x_{M} c_{v G, M}} \text {, and } \\
& \frac{\partial T_{S a t, M}}{\partial T_{G}}=T_{S a t, M}^{P} \frac{1}{T_{G}}
\end{aligned}
$$

Convergence is assumed when the absolute value of $G^{K}$ falls below an input criterion.

\section{E. Step 1 Derivatives}

Two subroutines are required for the mass-transfer models of step 1 of the AFDM algorithm where the SAEOS derivatives are both numerous and not necessarily straightforward. These subroutines are EOSVD1 for the single-phase cells, where the vapor volume is given by $\tilde{\alpha}$, and EOSTP for two-phase cells, where $\alpha_{\text {Geff }}$ is greater that $\tilde{\alpha}$.

There are 31 required derivatives for the EOSVD1 subroutine. These are defined in the FORTRAN array DER(IJ,N), where IJ is the cell number and $N$ is the derivative index. Because some of these derivatives are functions of other derivatives, to express these derivatives in terms of the SAEOS functions, the order of presentation is reversed. The 31 derivatives are written as

$$
\begin{aligned}
& \operatorname{DER}(\mathrm{IJ}, 31)=\frac{\partial \mathrm{T}_{\mathrm{Sat}, 2}}{\partial \mathrm{T}_{\mathrm{G}}}=\mathrm{T}_{\mathrm{Sat}, 2}^{\mathrm{p}} \frac{1}{\mathrm{~T}_{\mathrm{G}}}, \\
& \operatorname{DER}(\mathrm{IJ}, 30)=\frac{\partial \mathrm{T}_{\text {Sat,1 }}}{\partial \mathrm{T}_{\mathrm{G}}}=\mathrm{T}_{\mathrm{Sat}, 1^{\mathrm{p}}}^{\mathrm{p}} \frac{1}{\mathrm{~T}_{\mathrm{G}}}, \\
& \operatorname{DER}(\mathrm{IJ}, 29)=\frac{\partial \mathrm{T}_{\mathrm{Sat}, 2}}{\partial \bar{\rho}_{\mathrm{G} 2}}=\mathrm{T}_{\mathrm{Sat}, 2}^{\mathrm{p}} \frac{1}{\bar{\rho}_{\mathrm{G} 2}}, \\
& \operatorname{DER}(\mathrm{IJ}, 28)=\frac{\partial \mathrm{T}_{\mathrm{Sat}, 1}}{\partial \vec{\rho}_{\mathrm{G} 1}}=\mathrm{T}_{\mathrm{Sat}, 1}^{\mathrm{p}} \frac{1}{\bar{\rho}_{\mathrm{G} 1}},
\end{aligned}
$$




$$
\begin{aligned}
& \operatorname{DER}(\mathrm{IJ}, 27)=\frac{\partial \mathrm{e}_{\mathrm{vap}, 2}}{\partial \mathrm{T}_{\mathrm{G}}}=\left(\mathrm{c}_{\mathrm{vL}, 2}-\mathrm{R}_{2}\right) \frac{\partial \mathrm{T}_{\mathrm{Sat}, 2}}{\partial \mathrm{T}_{\mathrm{G}}} \\
& \operatorname{DER}(I J, 26)=\frac{\partial \mathrm{e}_{\mathrm{Vap}, 1}}{\partial \mathrm{T}_{G}}=\left(c_{\mathrm{vL}, 1}-R_{1}\right) \frac{\partial \mathrm{T}_{\mathrm{Sat}, 1}}{\partial \mathrm{T}_{G}}, \\
& \operatorname{DER}(\mathrm{IJ}, 25)=\frac{\partial \mathrm{e}_{\mathrm{Vap}, 2}}{\partial \bar{\rho}_{\mathrm{G} 2}}=\left(c_{\mathrm{vL}, 2}-\mathrm{R}_{2}\right) \frac{\partial \mathrm{T}_{\mathrm{Sat}, 2}}{\partial \bar{\rho}_{\mathrm{G} 2}}, \\
& \operatorname{DER}(\mathrm{IJ}, 24)=\frac{\partial e_{\mathrm{Vap}, 1}}{\partial \bar{\rho}_{\mathrm{G} 1}}=\left(c_{\mathrm{vL}, 1}-\mathrm{R}_{1}\right) \frac{\partial \mathrm{T}_{\mathrm{Sat}, 1}}{\partial \bar{\rho}_{\mathrm{G} 1}}, \\
& \operatorname{DER}(\mathbf{I J}, 23)=\frac{\partial e_{l_{g, 2}}}{\partial \mathrm{T}_{G}}=-R_{2} \frac{\partial \mathrm{T}_{\mathrm{Sat}, 2}}{\partial \mathrm{T}_{G}}, \\
& \operatorname{DER}(\mathrm{IJ}, 22)=\frac{\partial \mathrm{e}_{l \mathrm{~g}, 1}}{\partial \mathrm{T}_{\mathrm{G}}}=-\mathrm{R}_{1} \frac{\partial \mathrm{T}_{\mathrm{Sat}, 1_{1}}}{\partial \mathrm{T}_{\mathrm{G}}}, \\
& \operatorname{DER}(\mathrm{IJ}, 21)=\frac{\partial \mathrm{e}_{l_{g, 2}}}{\partial \bar{\rho}_{\mathrm{G} 2}}=-\mathrm{R}_{2} \frac{\partial \mathrm{T}_{\mathrm{Sat}, 2}}{\partial \bar{\rho}_{\mathrm{G} 2}}, \\
& \operatorname{DER}(\mathrm{IJ}, 20)=\frac{\partial \mathrm{e}_{l \mathrm{~g}, 1}}{\partial \bar{\rho}_{\mathrm{G} 1}}=-\mathrm{R}_{1} \frac{\partial \mathrm{T}_{S_{\mathrm{Sat}, 1}}}{\partial \bar{\rho}_{\mathrm{G} 1}}, \\
& \operatorname{DER}(\mathrm{IJ}, 19)=\frac{\partial \mathrm{e}_{\mathrm{Con}, 2}}{\partial \mathrm{T}_{\mathrm{G}}}=c_{\mathrm{vL}, 2} \frac{\partial \mathrm{T}_{\mathrm{Sat}, 2}}{\partial \mathrm{T}_{\mathrm{G}}}, \\
& \operatorname{DER}(\mathrm{IJ}, 18)=\frac{\partial \mathrm{e}_{\mathrm{Con}, 1}}{\partial \mathrm{T}_{\mathrm{G}}}=\mathrm{c}_{\mathrm{vL}, 1} \frac{\partial \mathrm{T}_{\mathrm{Sat}, 1}}{\partial \mathrm{T}_{\mathrm{G}}},
\end{aligned}
$$




$$
\operatorname{DER}(\mathrm{IJ}, 17)=\frac{\partial \mathrm{e}_{\mathrm{Con}, 2}}{\partial \bar{\rho}_{\mathrm{G} 2}}=\mathrm{c}_{\mathrm{vL}, 2} \frac{\partial \mathrm{T}_{\mathrm{Sat}_{2}, 2}}{\partial \bar{\rho}_{\mathrm{G} 2}},
$$

$\operatorname{DER}(\mathrm{IJ}, 16)=\frac{\partial \mathrm{e}_{\mathrm{Con}, 1}}{\partial \bar{\rho}_{\mathrm{G} 1}}=c_{\mathrm{vL}, 1} \frac{\partial \mathrm{T}_{\mathrm{Sat}, 1}}{\partial \bar{\rho}_{\mathrm{G} 1}}$,

$\operatorname{DER}(\mathrm{IJ}, 15)=\frac{\partial \mathrm{e}_{\mathrm{r}=4}}{\partial \mathrm{T}_{\mathrm{r}=4}}=c_{\mathrm{vL}, 2}$,

$\operatorname{DER}(\mathrm{IJ}, 14)=\frac{\partial \mathrm{e}_{\mathrm{r}=3}}{\partial \mathrm{T}_{\mathrm{r}=4}}=0$,

$\operatorname{DER}(I J, 13)=\frac{\partial e_{r=4}}{\partial T_{r=3}}=0$,

$\operatorname{DER}(\mathrm{IJ}, 12)=\frac{\partial \mathrm{e}_{\mathrm{r}=3}}{\partial \mathrm{T}_{\mathrm{r}=3}}=\mathrm{c}_{\mathrm{vL}, 1}$,

$\operatorname{DER}(\mathrm{IJ}, 11)=\frac{\partial \mathrm{e}_{\mathrm{I}=4}}{\partial \bar{\rho}_{\mathrm{L} 2}}=0$,

$\operatorname{DER}(\mathrm{IJ}, 10)=\frac{\partial \mathrm{e}_{\mathrm{T}=3}}{\partial \bar{\rho}_{\mathrm{L} 2}}=0$,

$\operatorname{DER}(\mathrm{IJ}, 9)=\frac{\partial \mathrm{e}_{\mathrm{T}=4}}{\partial \bar{\rho}_{\mathrm{L} l}}=0$,

$\operatorname{DER}(\mathrm{IJ}, 8)=\frac{\partial \mathrm{e}_{\mathrm{r}}=3}{\partial \bar{\rho}_{\mathrm{L} 1}}=0$,

$\operatorname{DER}(\mathrm{IJ}, 7)=\frac{\partial \mathrm{e}_{\mathrm{G} 2}}{\partial \mathrm{T}_{\mathrm{G}}}=\left(\mathrm{SC}_{2} \frac{\partial \mathrm{T}_{\mathrm{Sat}, 2}}{\partial \mathrm{T}_{\mathrm{G}}}+\mathrm{c}_{\mathrm{vG}, 2}\right.$, 


$$
\begin{aligned}
& \operatorname{DER}(\mathrm{IJ}, 6)=\frac{\partial \mathrm{e}_{\mathrm{Gl}}}{\partial \mathrm{T}_{\mathrm{G}}}=(\mathrm{SC})_{1} \frac{\partial \mathrm{T}_{\mathrm{Sat}_{1}, 1}}{\partial \mathrm{T}_{\mathrm{G}}}+\mathrm{c}_{\mathrm{vG}, 1} \\
& \operatorname{DER}(\mathrm{IJ}, 5)=\frac{\partial \mathrm{e}_{\mathrm{G} 2}}{\partial \bar{\rho}_{\mathrm{G} 2}}=(\mathrm{SC})_{2} \frac{\partial \mathrm{T}_{\mathrm{Sat}, 2}}{\partial \bar{\rho}_{\mathrm{G} 2}}, \\
& \operatorname{DER}(\mathrm{IJ}, 4)=\frac{\partial \mathrm{e}_{\mathrm{G} 1}}{\partial \bar{\rho}_{\mathrm{G} 1}}=(\mathrm{SC})_{1} \frac{\partial \mathrm{T}_{\mathrm{Sat}, 1}}{\partial \bar{\rho}_{\mathrm{G} 1}} \\
& \operatorname{DER}(\mathrm{IJ}, 3)=\frac{\partial \mathrm{e}_{\mathrm{G}}}{\partial \mathrm{T}_{\mathrm{G}}}=\frac{\bar{\rho}_{\mathrm{G} 1} \frac{\partial \mathrm{e}_{\mathrm{G} 1}}{\partial \mathrm{T}_{\mathrm{G}}}+\bar{\rho}_{\mathrm{G} 2} \frac{\partial \mathrm{e}_{\mathrm{G} 2}}{\partial \mathrm{T}_{\mathrm{G}}}+\bar{\rho}_{\mathrm{G} 3} \mathrm{c}_{\mathrm{vG}, 3}}{\bar{\rho}_{\mathrm{G} 1}+\bar{\rho}_{\mathrm{G} 2}+\bar{\rho}_{\mathrm{G} 3}} \\
& \operatorname{DER}(\mathrm{IJ}, 2)=\frac{\partial e_{\mathrm{G}}}{\partial \bar{\rho}_{\mathrm{G} 2}}=\frac{\bar{\rho}_{\mathrm{G} 2} \frac{\partial \mathrm{e}_{\mathrm{G} 2}}{\partial \bar{\rho}_{\mathrm{G} 2}}+\frac{-\bar{\rho}_{\mathrm{G} 1} \mathrm{e}_{\mathrm{G} 1}+\left(\bar{\rho}_{\mathrm{G}}-\bar{\rho}_{\mathrm{G} 2}\right) \mathrm{e}_{\mathrm{G} 2}-\bar{\rho}_{\mathrm{G} 3} \mathrm{e}_{\mathrm{G} 3}}{\bar{\rho}_{\mathrm{G}}}}{\bar{\rho}_{\mathrm{G}}} \text {, and } \\
& \operatorname{DER}(\mathrm{IJ}, 1)=\frac{\partial e_{\mathrm{G}}}{\partial \bar{\rho}_{\mathrm{G} 1}}=\frac{\bar{\rho}_{\mathrm{G} 1} \frac{\partial e_{\mathrm{G} 1}}{\partial \bar{\rho}_{\mathrm{G} 1}}+\frac{\left(\bar{\rho}_{\mathrm{G}}-\bar{\rho}_{\mathrm{G} 1}\right) \mathrm{e}_{\mathrm{G} 1}-\bar{\rho}_{\mathrm{G} 2} \mathrm{e}_{\mathrm{G} 2}-\bar{\rho}_{\mathrm{G} 3} \mathrm{e}_{\mathrm{G} 3}}{\bar{\rho}_{\mathrm{G}}}}{\bar{\rho}_{\mathrm{G}}} .
\end{aligned}
$$

In the two-phase subroutine EOSTP, the functions $\mathrm{h}_{\mathrm{g}, \mathrm{M}}$; $\mathrm{e}_{\mathrm{Lm}} ; \mathrm{T}$ Sat,M; $\mathrm{i}$ Con,M; $\mathrm{i}_{\mathrm{G}, \mathrm{M}}$; and $\mathrm{e}_{\mathrm{G}}$ must be differentiated with respect to the independent variables $\bar{\rho}_{\mathrm{Lm}}$; $\mathrm{T}_{\mathrm{Lm}} ; \mathrm{T}_{\mathrm{G}}$ and $\bar{\rho}_{\mathrm{GM}}$. The cases of both $\mathrm{M}=1$ and $\mathrm{M}=2(\mathrm{r}=3$ and 4, or $\mathrm{Lm}=\mathrm{L} 1$ and $\mathrm{L} 2)$ are considered.

Because the $h_{l g, M}$ are constants, all their derivatives are zero. The elm are also simple. The only nonzero terms existing from Eq. (2.3) are

$$
\frac{\partial e_{L m}}{\partial T_{L m}}=c_{v L, M} \text {, }
$$


where

$$
\begin{aligned}
& M=1 \text { corresponds to } L 1, \text { and } \\
& M=2 \text { corresponds to } L .2 .
\end{aligned}
$$

To get many of the remaining terms, the derivatives of the saturation temperatures must be known. The four saturation temperature derivatives with respect to the liquid densities (varying the subscripts $M$ and $m$ independently) are obtained by differentiating Eq. (2.23) as

$$
\frac{\partial \mathrm{T}_{\mathrm{Sat}, \mathrm{M}}}{\partial \bar{\rho}_{\mathrm{Lm}}}=\mathrm{T}_{\mathrm{Sat}, \mathrm{M}}^{\mathrm{p}} \frac{1-\alpha_{o}}{\alpha_{\mathrm{Geff}} \rho_{\mathrm{Lm}}}, \mathrm{M}=1,2 \text {, and } \ddot{\mathrm{Lm}}=\mathrm{L} 1, \mathrm{~L} 2
$$

The saturation temperature derivative with respect to the liquid temperatures are zero in the SAEOS because there is no direct dependence of the vapor partial pressures on the liquid temperatures. The saturation temperature derivatives with respect to the vapor temperature are given by Eqs. (2.51) and (2.52). The nonzero saturation temperature derivatives with respect to vapor densities are given by Eqs. (2.53) and (2.54). In AFDM, the assumption that components are immiscible makes the cross terms zero. Consequently, we have

$$
\frac{\partial \mathrm{T}_{\mathrm{Sat}_{1} 1}}{\partial \bar{\rho}_{\mathrm{G} 2}}=\frac{\partial \mathrm{T}_{\mathrm{Sat}, 2}}{\partial \bar{\rho}_{\mathrm{G} 1}}=0
$$

The derivatives of $\mathrm{i}_{\mathrm{Con}, \mathrm{M}}$ are evaluated next from Eq. (2.7). The four derivatives with respect to the liquid densities are

$$
\frac{\partial i_{\mathrm{Con}, \mathrm{M}}}{\partial \bar{\rho}_{\mathrm{Lm}}}=c_{\mathrm{vL}, \mathrm{M}} \frac{\partial \mathrm{T}_{\mathrm{Sat}, \mathrm{M}}}{\partial \bar{\rho}_{\mathrm{Lm}}}+\frac{\mathrm{p}_{\mathrm{GM}}}{\rho_{\mathrm{Lm}}} \frac{1-\alpha_{0}}{\alpha_{\mathrm{Geff}} \rho_{\mathrm{Lm}}}
$$

where

$$
\mathrm{M}=1 \text { and } 2 \text { such that } \mathrm{Lm}=\mathrm{L} 1 \text { and L2. }
$$


The derivatives of $\mathrm{iCon}_{\mathrm{M}} \mathrm{M}$ with respect to the liquid temperature are zero in the SAEOS. The vapor temperature derivatives are given by

$$
\frac{\partial \mathrm{i}_{\mathrm{Con}, M}}{\partial \mathrm{T}_{\mathrm{G}}}=\mathrm{c}_{\mathrm{vL}, \mathrm{M}} \frac{\partial \mathrm{T}_{\mathrm{Sal}, \mathrm{M}}}{\partial \mathrm{T}_{\mathrm{G}}}+\frac{\mathrm{p}_{\mathrm{GM}}}{\rho_{\mathrm{Lm}} \mathrm{T}_{\mathrm{G}}}, \mathrm{M}=1,2
$$

The only nonzero vapor density derivatives are those with respect to the same material (M) as iCon,M. This yields two expressions:

$$
\frac{\partial \mathrm{i}_{\mathrm{Con}, \mathrm{M}}}{\partial \bar{\rho}_{\mathrm{GM}}}=c_{\mathrm{vL}, \mathrm{M}} \frac{\partial \mathrm{T}_{\mathrm{Sat}, \mathrm{M}}}{\partial \bar{\rho}_{\mathrm{GM}}}+\frac{\mathrm{p}_{\mathrm{GM}}}{\rho_{\mathrm{Lm}} \bar{\rho}_{\mathrm{GM}}}, \mathrm{M}=1,2 .
$$

The individual vapor component enthalpies, $i_{G, M}$, from Eq. (2.12), are similarly dependent on the derivatives of the saturation temperatures. The four derivatives with respect to liquid densities are

$$
\frac{\partial \mathrm{i}_{\mathrm{G}, \mathrm{M}}}{\partial \bar{\rho}_{\mathrm{Lm}}}=(\mathrm{SC})_{\mathrm{M}} \frac{\partial \mathrm{T}_{\mathrm{Sat}, \mathrm{M}}}{\partial \bar{\rho}_{\mathrm{Lm}}}
$$

where

$$
\begin{aligned}
& M=1,2, \text { and } \\
& m=1,2, \text { independently. }
\end{aligned}
$$

The derivatives with respect to the liquid temperatures are zero. The derivatives with respect to the vapor temperature are

$$
\frac{\partial \mathrm{i}_{\mathrm{G}, \mathrm{M}}}{\partial \mathrm{T}_{\mathrm{G}}}=(\mathrm{SC})_{\mathrm{M}} \frac{\partial \mathrm{T}_{\mathrm{Sa}, \mathrm{M}}}{\partial \mathrm{T}_{\mathrm{G}}}+\mathrm{c}_{\mathrm{vG}, \mathrm{M}}+\mathrm{R}_{\mathrm{M}}, \mathrm{M}=1,2
$$

The two expressions giving the derivatives with respect to the vapor densities are 


$$
\frac{\partial \mathrm{i}_{\mathrm{G}, \mathrm{M}}}{\partial \bar{\rho}_{\mathrm{GM}}}=(\mathrm{SC})_{\mathrm{M}} \frac{\partial \mathrm{T}_{\mathrm{Sat}, \mathrm{M}}}{\partial \overline{\mathrm{\rho}}_{\mathrm{GM}}}, \mathrm{M}=1,2 .
$$

The final derivatives required are those with respect to the average vapor energy, $e_{G}$, evaluated using Eq. (2.11). The derivatives with respect to the liquid densities can be given by the mass average of the components. This yields

$$
\frac{\partial \mathrm{e}_{\mathrm{G}}}{\partial \bar{\rho}_{\mathrm{Lm}}}=\frac{\bar{\rho}_{\mathrm{G} 1}(\mathrm{SC})_{1} \frac{\partial \mathrm{T}_{\mathrm{Sat}, 1}}{\partial \bar{\rho}_{\mathrm{Lm}}}+\bar{\rho}_{\mathrm{G} 2}(\mathrm{SC}) \frac{\partial \mathrm{T}_{\mathrm{Sat}, 2}}{\partial \bar{\rho}_{\mathrm{Lm}}}}{\bar{\rho}_{\mathrm{G} 1}+\bar{\rho}_{\mathrm{G} 2}+\bar{\rho}_{\mathrm{G} 3}}, \mathrm{~m}=1,2 .
$$

The derivatives with respect to the liquid temperature are zero. Equations (2.79) through (2.81) give the expressions for the derivatives with respect to the vapor temperature and the vapor densities.

This completes the expression for the derivatives required for the EOSTP subroutine. As a final observation, to avoid oscillations, current programming of the SAEOS assumes that the single-phase branch of the EOS always will be taken in EOSVD1 and that EOSTP always will take the two-phase branch. For the complete specifications of these routines, see App. B. 


\section{THE TEOS}

\section{A. Assumptions for the TEOS}

1. Introduction. Although the TEOS in principle provides an exact model of the thermodynamics, there are some approximations in its implementation in the AFDM code. They are described in this chapter, along with the composition and usage of the set of tables. (See App. A, Table I, for the numbering of materials and subscripts. Here we use the default values of the material index $M^{\prime}$ for explaining the assignments.)

2. Composition of the TEOS. Figure 3.1 schematically shows the single-phase and two-phase regions of a pure substance in stable equilibrium (and also defines the terminology used hereafter). With reference to this diagram, the EOS tables in the AFDM code provide data for the following relations:*

- $\quad$ V Van and eVan as a function $\rho$ and $T$ over the liquid and vapor regions ("Van-der-Waals-type data"; for material Nos. 3, 2, and 1 only).

- PSId and esld as a function of $\rho$ and T over the solid region ("solid-EOS data"; fer material No. 5 only).

- $\quad \rho_{C o n}$ and $\rho$ Vap as a function of $T$ for the saturation curve, and PSat as well as econ and evap over this curve ("saturation data"; for material Nos. 3 and 2 only).

- $\quad$ Sus and $\rho_{\text {Suv }}$ as a function of $\mathrm{T}$ for the sublimation curve, and PSub as well as esus and esuv over the curve ("sublimation data"; for material Nos. 5 and 4 only). The vapor branch properties (subscript Suv) of the sublimation curve are not needed because there is no evaporization of solid materials in the code.

The data in the above tables are not restricted to the interior of the pertaining single-phase regions or two-phase boundary curves, respectively. Rather, they partially overlap and extend into neighboring coexistence regions, thus providing values for the metastable states of subcooling and overheating. For technical reasons the tables contain data that represent tension (negative pressure) or the unstable states between the spinodal limits of the Van-der-Waals-type EOS. It is up to the code to discriminate between states that are sensible and nonsensible in the

\footnotetext{
${ }^{*}$ Specific enthalpies $i$ are not tabulated but can be derived for $e, p$, and $\rho$ according to $i=e+p / \rho$.
} 


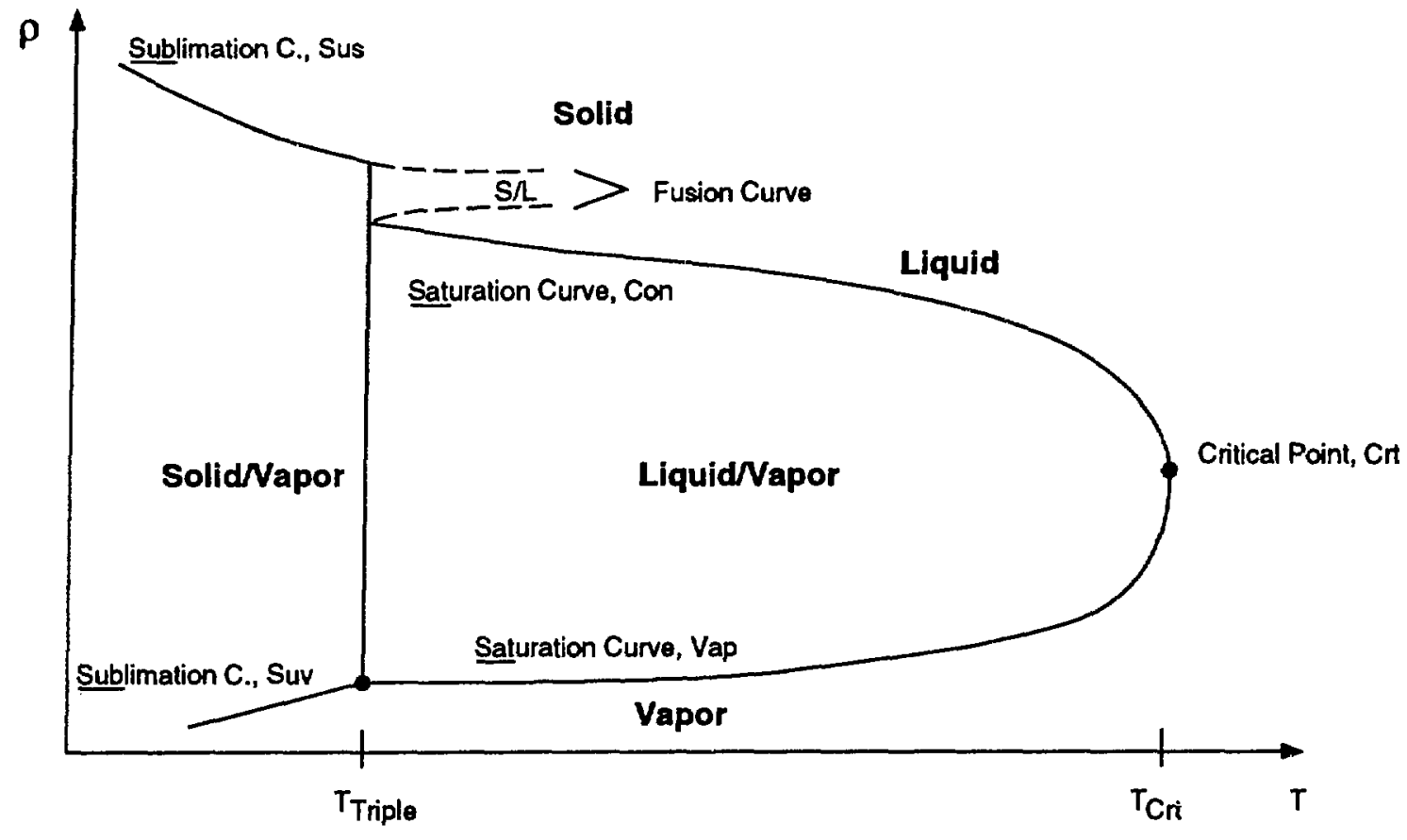

Fig. 3.1.

EOS plot over the $\rho-T$-plane for a pure material (not to scale). (Sub, Sus, Suv, Sat, Con, Vap, and Crt are subscripts used for the quantities.)

computational context. To enable the code to do so, an additional table containing the spinodal limits of the Van-der-Waals type data is provided in the code.

There are no tables for the "fusion curve" separating the solid/liquid twophase region from the single-phase solid and liquid regions because the only substance normally assumed to melt and freeze, represented by material Nos. 3 and 5 , is fuel, which as a mixture of substances has no unique fusion curve. Instead, in AFDM it is assumed that melting and freezing occurs independently of the pressure, above a solidus temperature $\mathrm{T}_{\mathrm{Sol}}$ and below a liquidus temperature $\mathrm{T}_{\mathrm{Liq}}$, both of which are input separately from the tables.

Thus, the diagram for the substance represented by material Nos. 3 and 5, as modeled in the AFDM code, looks like that depicted in Fig. 3.2 (for the substances represented by material Nos. 2,4, and 1, the undefined parts of the graph must be omitted).

3. Usage of the TEOS Data. The data of the above tables are used by the code according to the following rules: 


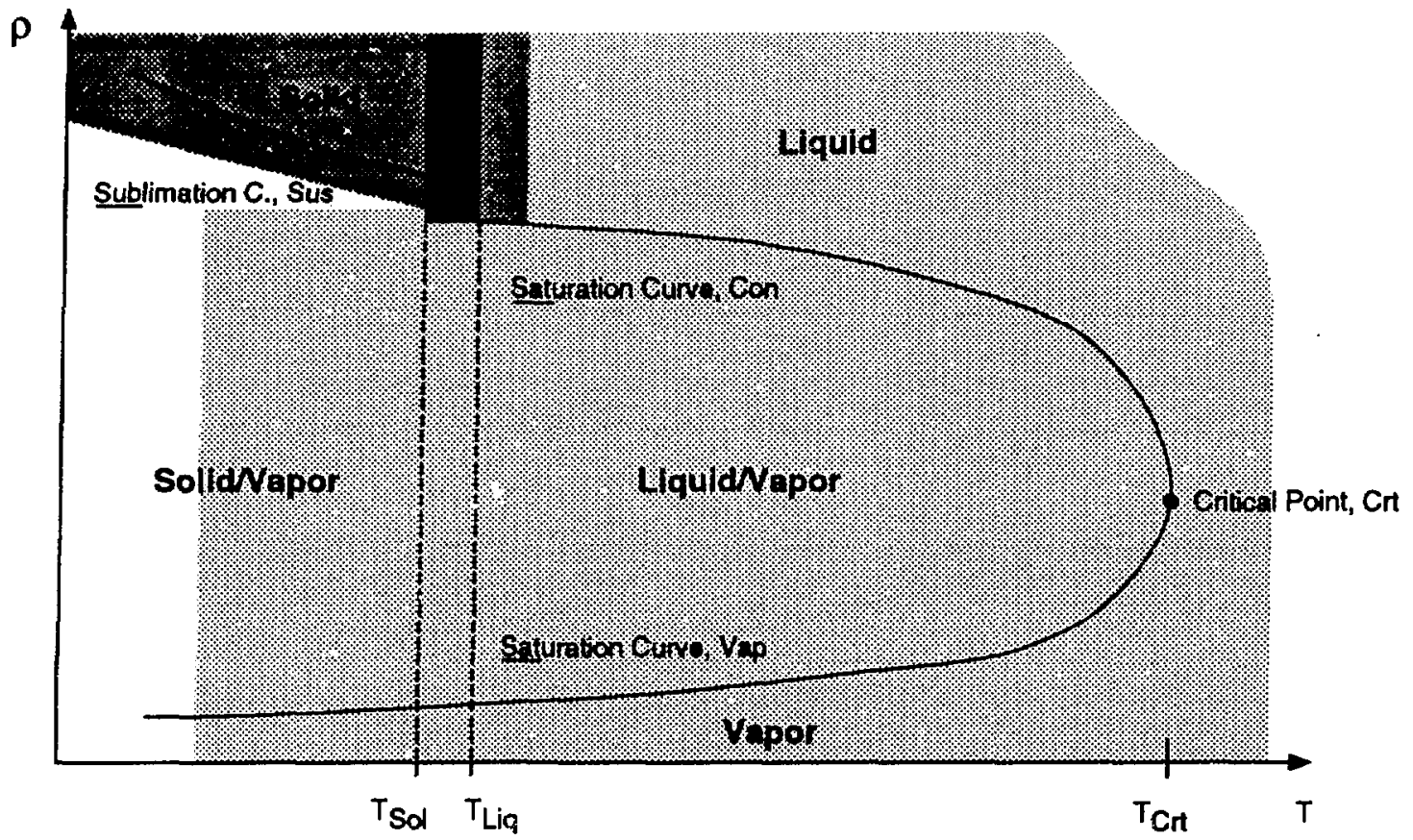

range for which Van-der-Waals type data are supplied (subscript Van)

range for which solid-EOS data are supplied (subscript Sld)

range for which the equilibrium state is defined

Fig. 3.2.

EOS plot over the $\rho$-T-plane for AFDM fuel modeling (not to scale).

(1) Pressure $p$ or specific internal energy e (and their derivatives) as a function of $\rho$ and $\mathrm{T}$ :

- taken from the Van-der-Waals-type table for component Nos. 3 through 7 (check for negative values and spinodal limits!):

$$
p_{m}=p V_{a n, M^{\prime}(m)}\left\{p_{m}, T_{r(m)}\right\} e_{m}=e V_{a n, M^{\prime}(m)}\left\{p_{m}, T_{r(m)}\right\} \text { for } m=3, \ldots, 7
$$

- taken from the solid-EOS table for component No. 2 (check for negative values!):

$$
\mathrm{p}_{2}=\operatorname{pSld}, 4\left(\rho_{2}, \mathrm{~T}_{2}\right\} \quad \mathrm{e}_{2}=\operatorname{esld}, 4\left\{\rho_{2}, \mathrm{~T}_{2}\right\}
$$


- There is no relation of the above kind for component No. 1 because it is assumed incompressible. The sublimation table is used in the initialization phase of the code to obtain the density $\rho_{1}$ as a function of the initial temperature $\left\{\mathrm{T}_{1}^{i}\right\}$ in each cell, which is then kept constant throughout the transient phase:

$$
\rho_{1}=\rho \operatorname{Sus}, 5\left\{T_{1}^{i}\right\}
$$

The sublimation table is also used to obtain the specific internal energy $e_{1}$ as a function of the temperature $T_{1}$ in all phases of the code:

$$
e_{1}=e_{S u s, 5}\left\{T_{1}\right\}
$$

(2) Saturation properties (and their derivatives) as a function of a vapor component's partial pressure, a liquid component's temperature, or a liquid component's specific internal energy:

- taken from the saturation table for component Nos. 3 through 6:

$$
\begin{aligned}
& \begin{array}{lll}
\text { PCon, } M^{\prime} & \text { for } & M=3,2 \text {, } \\
\rho V^{\prime} & \text { for } & M=3,2,
\end{array} \\
& \text { PSat, } M \text { for } M=3,2 \text {, } \\
& \text { TSat, } M^{\prime} \text { for } M=3,2 \text {, } \\
& \text { eCon, } M \text { for } M=3,2 \text {, and } \\
& \text { evap, } M \text { for } M=3,2 \text {. }
\end{aligned}
$$

From these properties the following quantities can be derived:

$$
\begin{aligned}
& \mathrm{e}_{\mathrm{g}, M^{\prime}}=\mathrm{eV}_{\mathrm{ap}, \mathrm{M}^{\prime}}-\mathrm{e}_{\mathrm{Con}, \mathrm{M}^{\prime}} \text { for } \mathrm{M}^{\prime}=3,2 \text {, } \\
& \mathrm{i}_{\mathrm{Con}, \mathrm{M}^{\prime}}=\mathrm{e}_{\mathrm{Con}, \mathrm{M}^{\prime}}+\mathrm{p}_{\mathrm{m}} / \rho_{\mathrm{Con}, \mathrm{M}^{\prime}} \quad \text { for } \quad \mathbf{M}^{\prime}=\mathbf{3 , 2} \text {, } \\
& i_{\text {Vap, }} M^{\prime}=\operatorname{eVap}_{,} M^{\prime}+p_{m} / \rho_{\text {Vap }, M^{\prime}} \quad \text { for } \quad M^{\prime}=3,2 \text {, and }
\end{aligned}
$$




$$
h_{1}, M^{\prime}=i V_{a p}, M^{\prime}-i \text { Con, } M^{\prime} \quad \text { for } \quad M^{\prime}=3,2 \text {. }
$$

- There are no relations of the above kind for component Nos. 1, 2, and 7.

(3) Critical properties:

- taken from the saturation table for component Nos. 3 through 6:

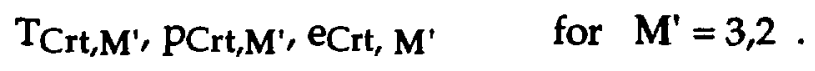

- There are no critical properties for component Nos. 1, 2, and 7.

(4) Fusion properties:

- For the substance represented by material Nos. 3 and 5, the solidus specific internal energy is taken from the sublimation table at the input solidus temperature; the liquidus specific internal energy is taken from the saturation table at the input liquidus temperature; the specific heat of fusion is the difference of the above energies:

$$
\begin{gathered}
\text { esol,1 }=e_{S u s, 5}\left\{T_{\text {Sol, }, 1}\right\} \\
e_{L i q, 1}=e_{C o n, 3}\left\{T_{L i q, 1}\right\} \text {, and } \\
h_{f, 1}=e_{L i q, 1}-e_{S o l, 1}
\end{gathered}
$$

These quantities are used in the melting/freezing model only.

- There are no fusion properties for the other materials.

4. Usage of Other EOS Data. Most of the quantities that are input and inputderived in the code option for the simplified analytic EOS are derived from the tables in the code option for the TEOS, such as initial densities, specific heat capacities, compressibilities, and the saturation, critical, and fusion properties mentioned in the previous section.

There are only the following thermodynamic quantities from the SAEOS input that must be supplied to the code in addition to the tables (compare App. D; note the relation between $M$ and $\mathbf{M}^{\prime}$ in Table A.I): 
- The superheats $\Delta T_{\text {Sup, }} M$, where $M=1,2$, are used in the initialization phase of the code (in calculating the equilibrium state of single-phase cells) and in the heat- and mass-transfer models.

- The solidus and liquidus temperatures $T_{S o l, M}$ and $T_{L i q, M}$ are required for $M=1$, as specified in the previous section. They are also used for $M=1,2$, as the temperatures assigned to component Nos. 1 through 4 and to derive the corresponding specific internal energies when zero volume fractions are input for these components:

$$
\begin{array}{ll}
\mathrm{T}_{1}^{0}=1 \times 10^{-2} \times \mathrm{T}_{\text {Sol, } 2} & \mathrm{e}_{1}^{0}=\mathrm{e}_{\text {Sus, } 4}\left\{\mathrm{~T}_{1}^{0}\right\}, \\
\mathrm{T}_{2}^{0}=\mathrm{T}_{\text {Sol, } 1} & \mathrm{e}_{2}^{0}=\mathrm{e}_{\text {Sus, } 5}\left\{\mathrm{~T}_{2}^{0}\right\}, \\
\mathrm{T}_{3}^{0}=\mathrm{T}_{\text {Liq, } 1} & \mathrm{e}_{3}^{0}=\mathrm{e}_{\text {Con }, 3}\left\{\mathrm{~T}_{3}^{0}\right\}, \text { and } \\
\mathrm{T}_{4}^{0}=\mathrm{T}_{\text {Liq, },} & \mathrm{e}_{4}^{0}=\mathrm{e}_{\text {Con, }, 2}\left\{\mathrm{~T}_{4}^{0}\right\}
\end{array}
$$

$\mathrm{T}_{\text {Liq, }} \mathrm{M}$, where $\mathrm{M}=1,2$, are also required in some other parts of the code, mainly in the heat- and mass-transfer models.

- The material gas constants $R_{M}, M=1,2,3$ are required to calculate the vapor mixture specific heat capacity at constant pressure. They should represent the gas constants at infinite dilution of the materials.

\section{B. Multimaterial Multiphase EOS Interface Model}

1. Introduction. The EOS interface models need to be sufficiently detailed to treat a mixture of three gas components, two liquid components, and one solid component. It is assumed that the two liquid components represent the liquid phase of the two gas components and that the particle component represents the solid phase for one of the two liquid components. All six material components and the structure component as the seventh component are depicted schematically in Fig. 3.3 in terms of macroscopic densities, temperatures, and volume fractions. This figure represents a typical AFDM numerical cell with the possibility of up to seven components residing within the cell. 


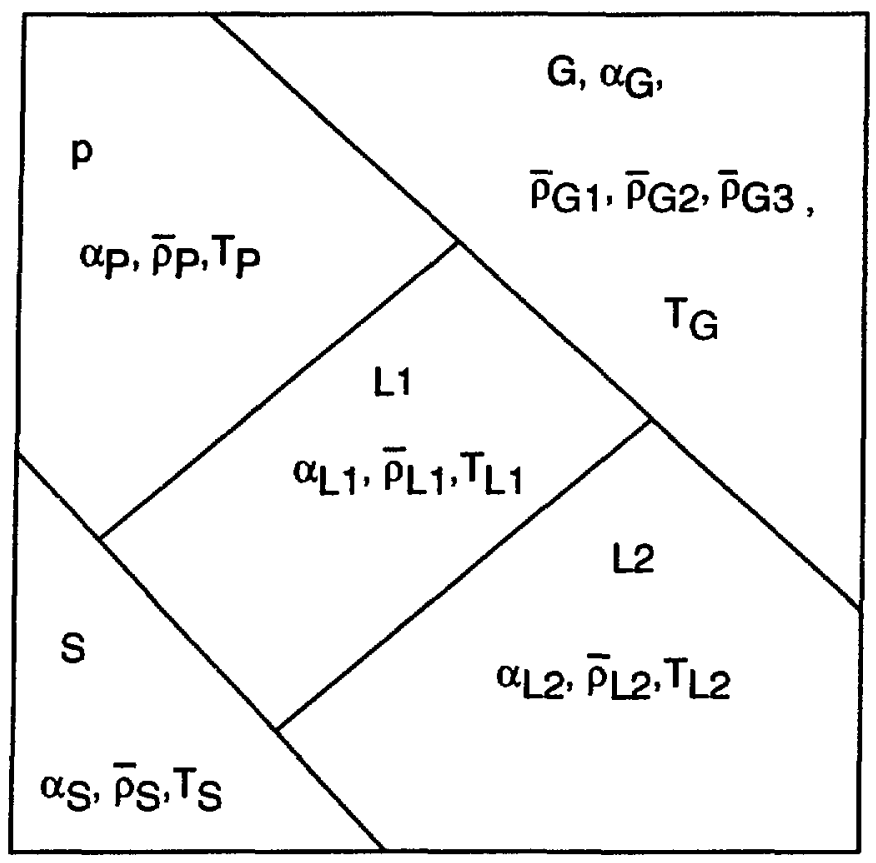

Fig. 3.3.

Components in an AFDM cell.

As indicated in Fig. 3.3, the set of independent variables with which the AFDM solution algorithm proceeds is composed of macrodensities and temperatures.

$$
\bar{x}=\left(\bar{\rho}_{p}, \bar{\rho}_{L 1}, \bar{\rho}_{L 2}, \bar{\rho}_{G 1}, \bar{\rho}_{G 2}, \bar{\rho}_{G 3}, T_{p}, T_{L 1}, T_{L 2}, T_{G}\right)^{T}
$$

where

$$
\mathrm{T}=\text { transposed }
$$

This vector represents the information normally available from the AFDM main routines for EOS interrogations. The subscript notation is written according to the notation used in Chap. II. The variables in this vector are determined essentially by the choice to use the conservative form of the conservation equations for mass, momentum, and energy. Note that an alternative form, where the pressure appears as an independent variable in the conservation equations, would be more 
convenient for the EOS interface because the mechanical equilibrium iteration could be avoided.

To determine the thermodynamic state of the cell from these input data, the following assumptions are made:

- The condensed components (two liquids, the particle components, and the structure) are immiscible.

- The gas components form a homogeneous mixture.

- The condensed components are in mechanical equilibrium (pressure equilibrium) with each other and with the gas phase.

There is usually neither thermal equilibrium nor phase equilibrium between the components in a cell. Otherwise, neither heat-transfer nor vaporization/condensation processes would take place. Howevor, pressure equilibrium is assumed to exist. The condensed components can be compressed beyond, or expand below, the saturated density until the mechanical equilibrium is reached. Then, if one includes the so-called $\alpha_{0}$ concept, which will be explained later, the thermodynamic state of the cell is completely defined by the input vector $\bar{X}$. This thermodynamic state is a unique and continuous function of each of the components of $\bar{X}$. However, discontinuities of the derivatives usually occur at the two-phase-to-single-phase transition and possibly at the so-called spinodal limits. Thus, although the principle of mechanical equilibrium requires costly iterative calculations, it certainly has the above-mentioned advantages.

Note that the structure component is not allowed to change its thermodynamic density. Therefore, this component is not included in the mechanical equilibrium calculations.

In some places of the AFDM code, inverted EOS evaluations are required. This means that in addition to the macrodensities, the internal energies of the four ( $P, L 1, L 2, G)$ energy components are given, and the temperatures must be calculated. This case will be discussed in Sec. 5 .

The multiphase, multimaterial EOS formalism presented in this manual is based on the above assumptions. The reference EOS in AFDM is the SESAME EOS library. Note, however, that the formalism would not change if an advanced analytical EOS were used. In the present AFDM version, an SAEOS (as described in Chap. II) is available. With this SAEOS, a simplified formalism is used where pressure equilibrium is calculated according to the outlines given in Chap. II. 
The following discussion is for the case that the SESAME library ${ }^{1-4}$ is used. In this model description, Sec. 2 presents the general features of the EOS for one material, as applied to the AFDM EOS interfaces. Section 3 describes the detail of mechanical equilibrium states that serves as the basic principle of the AFDM EOS interface algorithm. Sections 4 and 5 describe the saturation properties and a method to invert internal energies into the temperatures. Section 6 derives the various partial derivatives of the EOS functions with respect to the AFDMindependent variables.

2. Material Equations of State

a. Basic Relations. Multicomponent, multiphase EOS interface theory relates the smear densities (called macrodensities in AFDM) and temperatures to the thermodynamic densities (called microdensities), pressure, and internal energies. Furthermore, such EOS information as partial derivatives of EOS-dependent variables is required for the AFDM exchange function models to predict the mass and heattransfer rates and the AFDM pressure iteration scheme.

In most cases, the EOS interface routines receive the macrodensities and temperatures that are calculated in the AFDM thermohydraulic calculations. Also, the AFDM algorithms require an inverse temperature calculation with the macrodensities and the given internal energies.

Fundamental thermodynamic relationships among material state variables are:

$$
\begin{aligned}
& p_{m}=p\left(\rho_{m}, T_{m}\right), \text { and } \\
& e_{m}=e\left(\rho_{m}, T_{m}\right),
\end{aligned}
$$

where $p$ is the thermodynamic pressure, $p$ is the microdensity, $T$ is the temperature, and $e$ is the specific internal energy. Subscript $m$ denotes the material component. In addition to pressure and internal energy, the following partial derivatives are also required:

$$
\left(\frac{\partial p}{\partial \rho}\right)_{T},\left(\frac{\partial p}{\partial T}\right)_{\rho},\left(\frac{\partial e}{\partial \rho}\right)_{T} \text {, and }\left(\frac{\partial e}{\partial T}\right)_{\rho} \text {, }
$$

The macroscopic density (macrodensity) of the m-th component $\bar{\rho}_{\mathrm{m}}$ is related to the microdensity $\rho_{m}$ with the volume fraction $\alpha_{\mathrm{m}}$, where 


$$
\alpha_{\mathrm{m}}=\bar{\rho}_{\mathrm{m}} / \rho_{\mathrm{m}}
$$

The vapor volume fraction $\alpha_{G}$ is defined from volume balance, which requires

$$
\alpha_{G}=1-\Sigma \alpha_{m}, m=S, P, L 1, \text { and } L 2
$$

Because of the desire for the single-phase-to-two-phase transition to take place at some finite vapor volume fraction for the pressure iteration to interface with the EOS, the AFDM EOS interface routines employ a slightly modified version of Eqs. (3.2) and (3.3). This will be discussed in detail in Sec. 3.b.

The microdensities, and thus, the volume fractions, are the result of the multicomponent mechanical equilibrium calculations, as will be discussed in Sec. 3 . Then, with the component microdensity and temperature given, it is possible to calculate the partial derivatives of the EOS-dependent variables.

b. Vapor Mixture Rule. While it is assumed that two liquid components and one particle component are immiscible, three vapor/gas components are assumed to form a homogeneous mixture described by the Gibbs-Dalton rule. This mixture rule is constrained to give an ideal mixing for ideal gases, but it would give reasonable results for nonideal gases, as well.

The total gas pressure is equal to the sum of the individual gas partial pressures,

$$
\mathrm{p}_{\mathrm{G}}=\sum_{\mathrm{M}=1}^{3} \mathrm{p}_{\mathrm{GM}}\left(\rho_{\mathrm{GM}}, \mathrm{T}_{\mathrm{G}}\right)
$$

and the gas mixture internal energy is equal to a mass-fraction weighted sum of the individual gas internal energies:

$$
e_{G}=\sum_{M=1}^{3} x_{M} e_{G M}\left(\rho_{G M}, T_{G}\right)
$$

where

$$
X_{m}=\bar{\rho}_{G M} / \sum_{M=1}^{3} \bar{\rho}_{G M} .
$$


PGM is the thermodynamic (or microscopic) density of the vapor phase component $\mathrm{GM}(\mathrm{M}=1,2,3)$ and is defined as $\rho_{\mathrm{GM}} / \alpha_{\mathrm{Geff}}$, where $\alpha_{\mathrm{Geff}}$ is an effective vapor volume fraction described later in Sec. 3.a. $\rho_{G M}$ is available directly from the EOS table or analytical EOS once the gas microdensities are fixed in the mechanical equilibrium calculations.

c. Van-der-Waals-Type Equation of State. Figure 3.4 shows two isotherms (below the critical temperature) of a Van-der-Waals-type EOS. Such an isotherm is composed of five portions:

- the compressed liquid $A B$,

- the superheated liquid $B C$,

- the unstable region $C D$,

- the subcooled vapor $\mathrm{DE}$, and

- the superheated vapor EF.

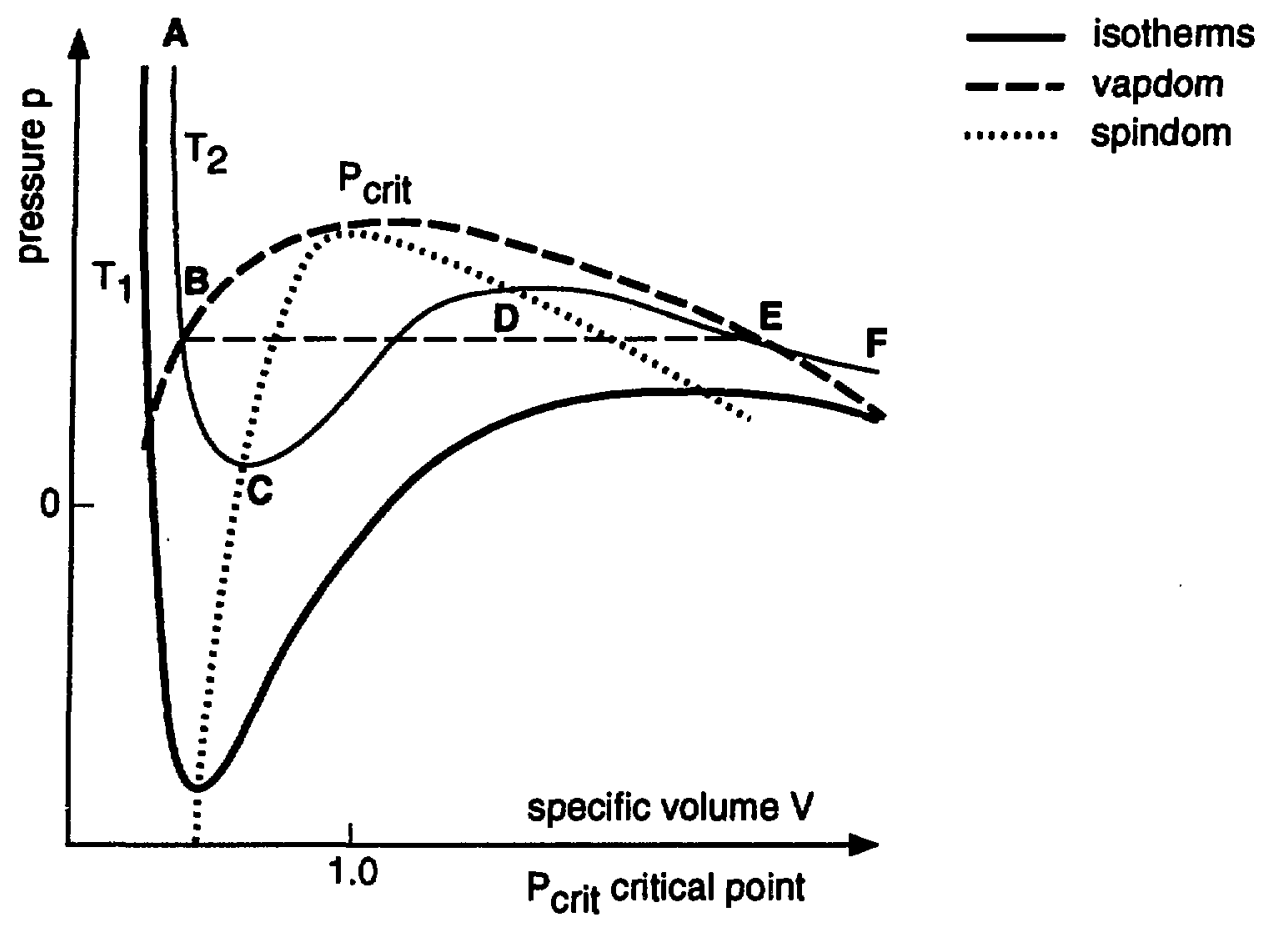

Fig. 3.4.

Two isotherms of Van-der-Waals-type EOS. 
The saturated liquid and vapor volumes at positions B and D, respectively, are obtained by Maxwell's equal area rule. In phase equilibrium, saturated liquid and vapor coexist, and the pressure is constant (dashed line in Fig. 3.4). The saturation curve is also called the vapor dome.

The part BC of the isotherm is metastable in the sense that superheated liquid exists if there are no nucleation sites. In the presence of nucleation sites, the liquid evaporates. These states are not in thermodynamic equilibrium. The same holds for the region $\mathrm{DE}$. The part $\mathrm{CD}$, however, is thermodynamically unstable because in this region, where $\partial p / \partial \rho<0$, an expansion would lead to a pressure increase. Thus, states in this part cannot occur.

The limit points $\mathrm{C}$ and $\mathrm{D}$ are called spinodal points. For variable temperature, they form the spinodal curve, which passes through the critical point (see also Sec. III.E.2). Because there is usually no phase equilibrium between a liquid and its vapor, the liquid can be in a state along $A B C$, and the vapor can be in a state along DEF. For the EOS interface, this means that the states of liquid and gas are on the Van-der-Waals loop, not on the coexistence straight line; the EOS is required to have the superheated and subcooled parts.

While most SESAME tables have the Van-der-Waals loops, the SAEOS does not. Therefore, with the latter, two-phase mechanical equilibrium calculations are not possible. It is desirable for any improved analytical EOS to have the Van-derWaals loops, as well.

d. The SESAME EOS Library. The reference EOS database for AFDM is the SESAME EOS library of tabulated data and interpolation software.5,6 SESAME is a standardized, computer-based library that contains tables of thermodynamic properties for a wide range of material over a wide range of physical regions. The library currently contains data for about 70 materials, including metals, minerals, polymers, mixtures, and simple atomic and molecular species.

In the context of this manual, two types of SESAME tables (see Secs. III.D and III.E) are referred to: a "301" data table and a "401" data table. The 301 table contains pressure $p$, internal energy $e$, and Helmholtz free energy data as a function of density $\rho$ and temperature $T$ over the subcooled and superheated liquid and superheated and supersaturated vapor physical regions. The 401 table contains saturation dome data tabulated along the liquid and vapor saturation curves. These two tables and the inverted form of the 301 table form, together with the newly implemented solid data, the EOS database for the AFDM code. The SESAME formalism for accessing, manipulating, and interpolating these tables is the standard AFDM EOS 
treatment. For some reason, the TEOS data may not have the desired level of accuracy for sodium, a plutonium-uranium-dioxide mixture, and stainless steel. Once accurate data for these materials are collected from the various sources, new data tables can be constructed using one of two possible approaches.

1. The EOS tables (301 and 401) that form the basis of the SESAME formalism can be directly loaded with the more accurate data.

2. The theory for creating SESAME EOS surfaces uses a number of free parameters that tune the EOS surface to match experimental data. This is the more common approach for adjusting the SESAME EOS to agree with experimentally determined critical points, saturation curve data, etc. The EOS utility GRIZZLY7 is one of the tools used for such tuning operations.

Several theoretical models are used in generating EOS tables for the SESAME library. A particularly useful method for reactor safety applications is that of Barnes and DeVault. 8,9 The treatment is similar to a virial expansion; however, the pressure as a function of density $\rho$ and temperature $T$ is written as

$$
\mathrm{P}(\rho, T)=\mathrm{Pc}_{c}(\rho)+R T\left[\rho+b(T) \rho^{2}+c(T) \rho^{3}\right]
$$

where $p_{c}(\rho)$ is the zero-K curve, which includes the effects of forces between the atoms in a static configuration. Temperature-dependent terms represent the pressure arising from thermal motion of the atoms. The functions $b(T)$ and $c(T)$ are obtained by matching this expression to a modified Debye model for the crystal at normal density. As shown in Fig. 3.4, the calculated isotherms have a Van-derWaals form below an implied critical temperature. States between points B and C are thermodynamically unstable and are not allowed.

3. Mechanical Equilibrium. In the following sections in this chapter, the methods to obtain the cell equilibrium pressure and the thermodynamic properties of all the components are described. The basic principle to get these quantities is based on the assumption of mechanical equilibrium among the components. The mechanical equilibrium is said to be achieved when a thermodynamic pressure is unique to all the constituents of the cell. Here, vapor and noncondensable mixture are considered to be one constituent. Thus, a total of vapor partial pressure is equal to the pressure of the other constituents. The liquid/particle components with an 
extremely small macrodensity would not contribute to the mechanical equilibrium and are ignored in calculating the cell pressure ("missing components").

a. Basic Concept. The EOS concept is based on "mechanical equilibrium" in a cell (as described in Chaps. I and II), which means that all three condensed components are in pressure equilibrium with each other and with the vapor mixture. In addition, we assume that the two-phase-to-single-phase transition (as a function, for example, of one of the liquid macrodensities) occurs at a fictitious vapor volume fraction that is connected with $\alpha_{0}$. This concept is physically sound and leads to a well-defined state of the cell, including the transition point two-phase-to-singlephase, which is a continuous function of each variable of the input vector $\overline{\mathrm{X}}$. Under these conditions, the liquids are not at their saturation densities but can expand or compress, depending on the equilibrium cell pressure. A definite disadvantage of this concept is that time-consuming iterations are necessary to obtain the equilibrium liquid densities, which in two-phase cells (at temperatures well below critical) are usually very close to the saturation densities. This concept leads to the equation

$$
\mathrm{p}\left(\rho_{\mathrm{P}}, \mathrm{T}_{\mathrm{P}}\right)=\mathrm{p}\left(\rho_{\mathrm{L} 1}, \mathrm{~T}_{\mathrm{L} 1}\right)=\mathrm{p}\left(\rho_{\mathrm{L} 2}, \mathrm{~T}_{\mathrm{L} 2}\right)=\mathrm{p}\left(\rho_{\mathrm{G}}, \mathrm{T}_{\mathrm{G}}\right)
$$

where the thermodynamic densities, or microdensities $\rho$, are related to the volume fractions and the macrodensities by Eq. (3.2). Thus, given the macrodensities and the temperatures, there are three equations [Eq. (3.6)] for the four $\alpha$, which are supplemented by the volume balance equation

$$
\alpha_{G}=1-\alpha_{S}-\alpha_{P}-\alpha_{L 1}-\alpha_{L 2}
$$

Note that the structure is treated as incompressible and is therefore not included in the mechanical equilibrium calculation.

Equation (3.7) defines the true physical gas volume fraction, at least for twophase states of a cell. To make sure that the transition between the two phase and the single phase occurs at a well-defined nonzero pressure, which is a continuous function of the input variables also at the transition point, an effective gas volume

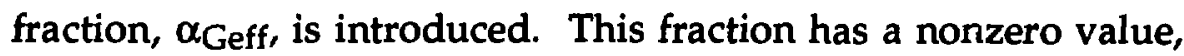

$$
\tilde{\alpha}=\alpha_{0}\left(1-\alpha_{s}\right) \text {. }
$$


if the condensed components occupy the complete volume (i.e., if $\alpha_{G}$ of Eq. (3.7) is zero). Note that $\tilde{\alpha}$ is a given fraction, $\alpha_{0}$, of the volume that is available for the compressible components. Then, $\alpha_{\mathrm{Geff}}$ can be defined by

$$
\alpha_{\mathrm{Geff}}=\max \left[\tilde{\alpha}, 1-\alpha_{s}-\left(1-\alpha_{0}\right)\left(\alpha_{p}+\alpha_{L 1}+\alpha_{L 2}\right)\right] .
$$

Figure 3.5 illustrates the relationship between $\alpha_{\mathrm{G}}$ and $\alpha_{\mathrm{Geff}}$.

With this definition, whether a cell is in a two-phase or single-phase state can clearly be decided. First, a two-phase mechanical equilibrium calculation must be carried out for all cells. It may occur, according to Eq. (3.8), that the sum of the condensed component is larger than one. In this case, $\alpha_{G e f f}=\tilde{\alpha}$, and the cell is in a single-phase state. The two-phase iteration calculates the pressure $\mathrm{pG}(\tilde{\alpha})$ in this case. Then, the components $P, L 1$, and L2 must be compressed to a higher pressure state, where their volume fractions add up to $1-\alpha_{s}$. In the other case, $\alpha_{\text {Geff }}>\tilde{\alpha}$, and the cell is in a two-phase state.

Figure 3.6 shows the gas volume fractions and the cell pressure as a function of the liquid content of the cell; $\overline{\mathrm{P}}_{\mathrm{G}}$ and the temperatures are constant. The variable

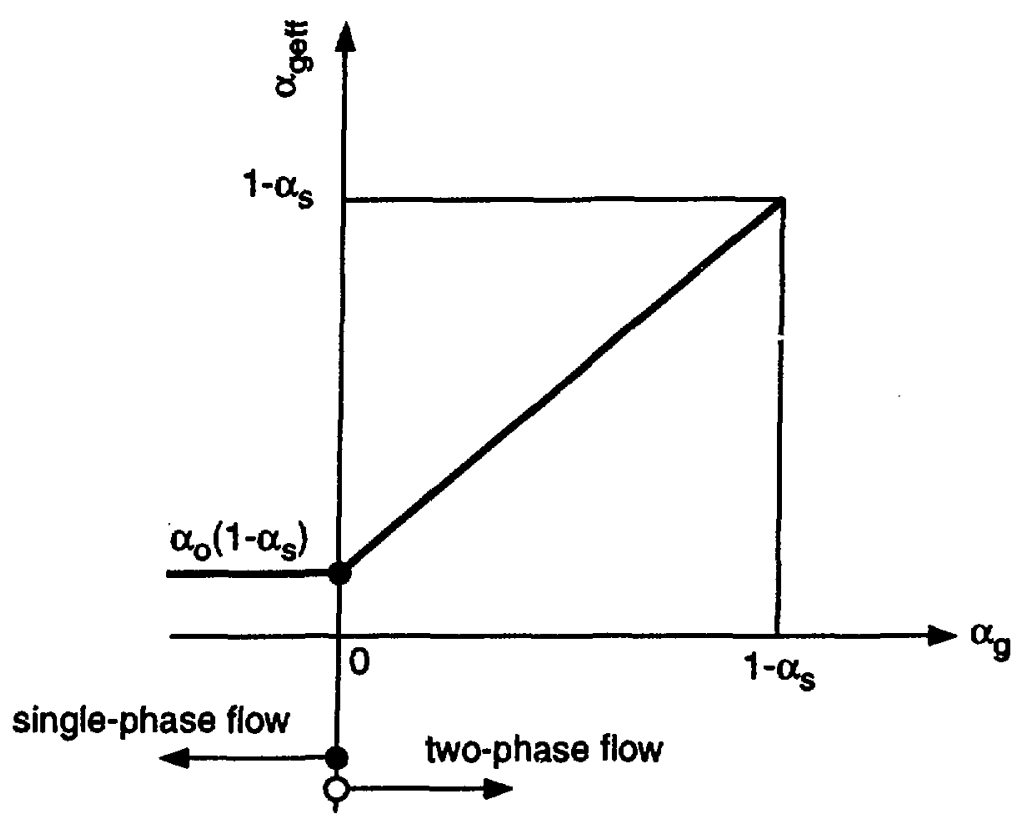

Fig. 3.5.

Effective-vs-physical gas volume fraction. 


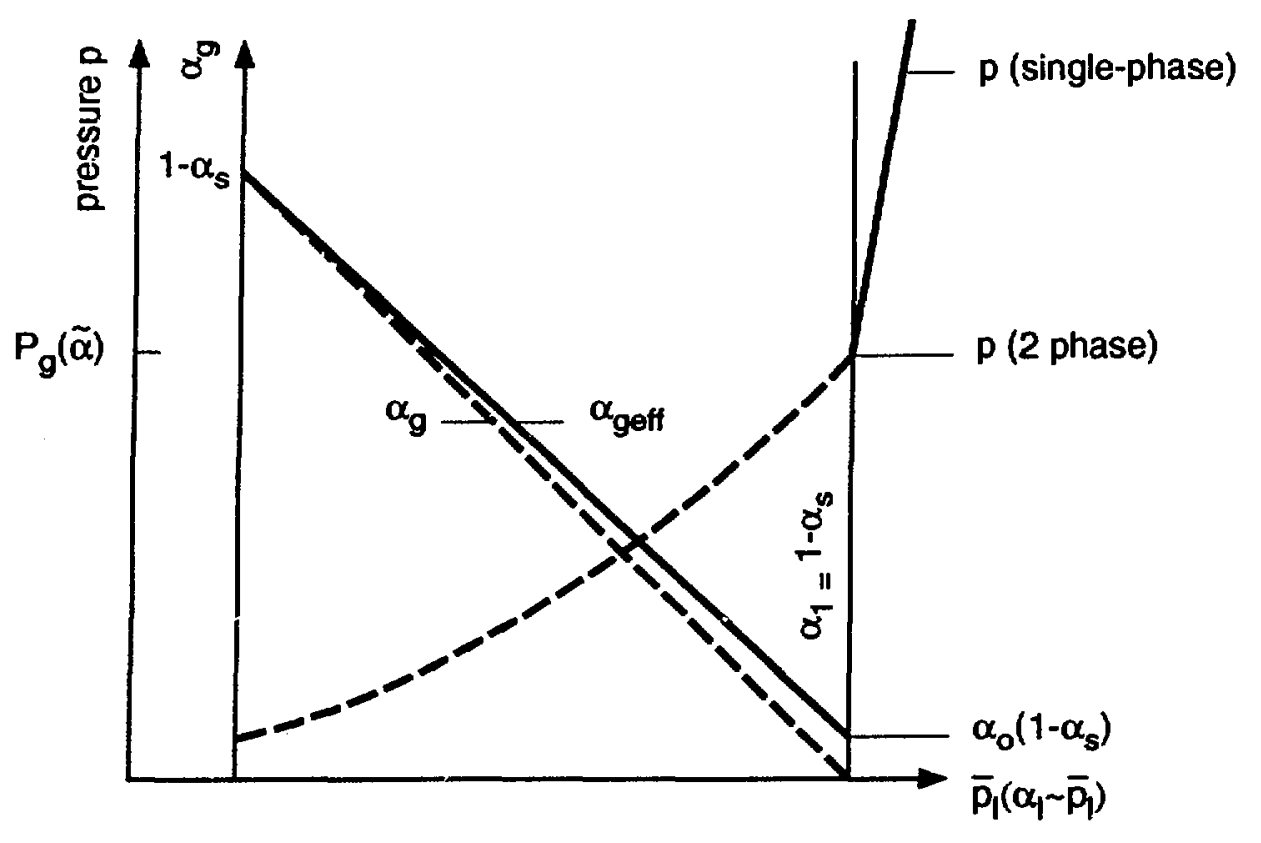

Fig. 3.6.

Gas volume fraction and pressure-vs-liquid mass in a cell.

$\bar{\rho}_{L}$ stands for the macrodensity of the liquid and is a suitable average if the cell contains more than one condensed material. Then, $\bar{\rho}_{L}$ determines essentially the volume fractions of the liquid and the gas. Figure 3.6 shows both $\alpha_{G}$ and $\alpha_{G e f f}$. The macrodensity $\bar{\rho}_{\mathrm{G}}$ then determines the two-phase cell pressure.

If $\alpha_{\mathrm{L}}$ exceeds the value $1-\alpha_{\mathrm{S}}$, the gas volume fraction $\alpha_{\mathrm{Geff}}$ and the twophase cell pressure $\mathrm{pG}(\tilde{\alpha})$ are constant. In this case, a single-phase pressure equilibrium state must be determined where the three components $P, L 1$, and L2 are compressed such that their volume fractions add up to $1-\alpha_{s}$. The (compressed) singlephase volume fractions are called $\alpha^{*}$. Thus, for the equilibrium state,

$$
\sum_{m} \alpha_{m}^{*}=1-\alpha_{s}
$$

where

$$
\mathrm{m}=\mathrm{P}, \mathrm{L} 1,2 .
$$


The single-phase pressure usually increases very steeply for increasing $\bar{\rho}_{L^{\prime}}$ as indicated in Fig. 3.6 (note that the volume fractions $\alpha^{*}$ are not shown in the figure). This pressure is always larger than the pressure $\mathrm{pG}(\tilde{\alpha})$ that resulted from the twophase iteration.

With this procedure, the cell pressure is continuous at $\alpha_{\mathrm{L}}=1-\alpha_{\mathrm{S}}$; the value $p_{G}(\tilde{\alpha})$ is the "base pressure" for the single-phase compression. It is important to realize that this base pressure has nothing to do with the saturation pressure of the liquids (no phase equilibrium) but depends on the gas content and gas temperature.

b. Treatment of Missing Components. For those liquid/particle components that have a very small macrodensity, the changes in their microdensities with respect to changes in variables influencing the pressure will be negligible in an absolute sense. Therefore, it is a reasonable assumption to ignore the components in the mechanical equilibrium calculations that contribute insignificantly to the pressure. A currently proposed criterion is to ignore the components with macrodensity less than $10^{-10} \mathrm{~kg} / \mathrm{m}^{3}$ (defined as "missing components").

Because the missing liquid/particle components are assumed not to contribute to the mechanical equilibrium, their microdensities and internal energies are defined in a different way from the others. In the AFDM EOS interfaces, $\bar{\rho}_{m}$ is set to $10^{-20} \mathrm{~kg} / \mathrm{m}^{3}$ if the input was zero. In that case, the liquidus temperatures for L1 and/or $\mathrm{L} 2$ are assigned to $\mathrm{T}_{\mathrm{Lm}}$, and the solidus temperature is assigned to TP. For these, as well as for the missing components $\left(10^{-20}<\bar{p}_{\mathrm{m}} \leq 10^{-10} \mathrm{~kg} / \mathrm{m}^{3}\right)$, the relation between the component temperatures $T_{m}$ and the component densities and specific internal energies is given by the saturation tables for the liquid and the sublimation table for particle components. The liquidus and solidus temperatures are input data.

It is also suggested that the partial derivatives associated with the missing components should be zeroed, except for the derivatives of the internal energyrelated variables with respect to the missing component temperature. Note that there is no missing component for the vapor phase. Even if an $\mathbf{M}^{\text {th }}$ vapor component has $\bar{\rho}_{\mathrm{GM}}$ less than $10^{-10} \mathrm{~kg} / \mathrm{m}^{3}$, we assume that all the vapor components contribute to the two-phase mechanical equilibrium. This was discussed briefly in Sec. 2.b and will be further explained in Sec. 3.f. In the context that follows in this chapter, the missing components are totally excluded from these considerations.

c. EOS Function Limits. There are two kinds of limits to be imposed on the EOS functions; (1) minimum and maximum thermodynamic densities and temperatures that limit the availability of either analytical EOS or tabular EOS, and (2) critical temperature and pressure when saturation properties are of interest 
under the vapor dome. It is noted that the missing components are excluded from the following discussions.

- Limits from the Availability of the Data For example, when the SESAME 301 data tables are interrogated, the following overall temperature limits are first checked:

$-0.0 \leq \mathrm{T}_{\mathrm{Na}} \leq 10155 \mathrm{~K}$,

$-297.1 \leq$ TUO2 $\leq 17407 \mathrm{~K}$, and

$-297.1 \leq$ TSS $_{\text {SS }} \leq 11605 \mathrm{~K}$.

The related density limits are (see also App. F)

$-0.0 \leq \rho_{\mathrm{Na}} \leq 1.31 \mathrm{~g} / \mathrm{cm}^{3}$,

$-0.0 \leq$ PUO2 $\leq 10.97 \mathrm{~g} / \mathrm{cm}^{3}$, and

$-0.0 \leq \rho_{\mathrm{SS}} \leq 7.87 \mathrm{~g} / \mathrm{cm}^{3}$.

\section{- Critical Temperature and Pressure}

When saturation properties are to be obtained, the temperature or pressure should be below critical point values (SESAME 401 data):

$-\quad 0.0 \leq \mathrm{T}_{\mathrm{Na}}<2539 \mathrm{~K}$,

- $297.1 \leq$ TUO2 $\leq 13530 \mathrm{~K}$, and

- $297.1 \leq \mathrm{T}_{\mathrm{SS}} \leq 8251 \mathrm{~K}$,

and

$-0.0 \leq \mathrm{PNa}_{\mathrm{Na}}<0.0223 \mathrm{GPa}$,

$-3.7^{*} 10^{-11} \leq$ PUO2 $<0.8427 \mathrm{GPa}$, and

$-3.6^{* 10^{-11}} \leq$ PSS $<0.3449 \mathrm{GPa}$.

For more details, see App. F. However, it is noted that $T_{\max }, \rho_{\max }, \mathrm{pCrt}_{\mathrm{C}}$, and $\mathrm{T}_{\mathrm{Crt}}$ are subject to change whenever the table data are updated. Note also that the lower limits depend on the tables used. Birational function interpolation will cause problems for $\mathrm{UO}_{2}$ data if a data point is between the first two points 
of the table. This interpolation should be improved for the next updated version in the same manner as for sodium and stainless-steel data (see App. F).

d. Two-Phase Mechanical Equilibrium Calculation. According to the concept of the effective vapor volume fraction, as discussed in Sec. 3.a, it is necessary to carry out the two-phase mechanical (pressure) equilibrium calculation for all cells. Therefore, the routine EOSPEQ first carries out this iterative calculation. After the calculation has converged, the volume fractions are available that are needed to decide whether a cell is a two-phase or single-phase flow cell. The major assumptions for the two-phase equilibrium are:

1. Missing components do not take part in the pressure equilibrium.

2. The vapor phase occupies the effective volume fraction $\alpha_{\text {Geff }}$. This fraction cannot drop below $\tilde{\alpha}$.

3. The vapor content of a single-phase cell, where $\alpha_{G \text { eff }}=\tilde{\alpha}$, cannot decrease because there is no condensation in single-phase cells within the EOS.

In EOSPEQ, the equations are programmed for the general case where all three condensed components, P, L1, and L2, are present. These equations will now be derived. Simplifications that occur when one or two components are missing will be discussed later:

The conditions of pressure equilibrium between each of the condensed components $m$ and the vapor phase are

$$
\begin{aligned}
\mathrm{p}=(\text { two phase }) & =\sum_{M=1}^{3} p_{G M}\left(\rho_{G M}, T_{G}\right), \\
& =p\left(\rho_{p}, T_{p}\right), \\
& =p\left(\rho_{L 1}, T_{L 1}\right), \text { and } \\
& =p\left(\rho_{L 2}, T_{L 2}\right) .
\end{aligned}
$$

In this section, $m$ stands for any of the three condensed components $P, L 1$, and L2. The microdensities are related to the volume fractions by

$$
\rho_{m}=\frac{\bar{\rho}_{m}}{\alpha_{m}} \text {, and }
$$




$$
\rho_{\mathrm{GM}}=\frac{\bar{\rho}_{\mathrm{GM}}}{\alpha_{\mathrm{Geff}}},
$$

and $\alpha_{G e f f}$ is defined by

$$
\alpha_{\mathrm{Geff}}=\max \left[\tilde{\alpha}, 1-\alpha_{s}-\left(1-\alpha_{0}\right) \sum \alpha_{\mathrm{m}}\right]
$$

The above equations must be solved for given temperatures $T$ and macroscopic densities $\rho$. Through the relation (3.10), the $\alpha$ 's can be introduced as variables instead of the $\rho$. Then, one has three equations [Eq. (3.9)] for the four variables $\alpha_{p}$,

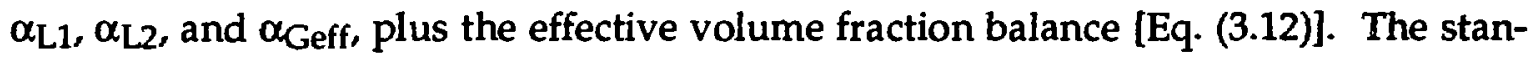
dard Newton-Raphson iteration procedure is used for the solution. The conditions for the iterative step $k+1$ are

$$
\sum_{M=1}^{3} p_{G}\left(\rho_{G M}^{k+1}\right)-p\left(\rho_{m}^{k+1}\right)=0
$$

where

$$
\rho^{k+1}=\rho^{k}+\Delta \rho
$$

This leads to

$$
\sum_{M=1}^{3} p_{G}\left(\rho_{G M}^{k}\right)+\sum_{M=1}^{3}\left(\frac{\partial p_{G}}{\partial \rho_{G M}}\right)^{k} \Delta \rho_{G M}=p\left(\rho_{m}^{k}\right)+\left(\frac{\partial p}{\partial \rho_{m}}\right)^{k} \Delta \rho_{m}
$$

From the relation (3.10), one has

$$
\frac{\Delta \rho}{\rho}=-\frac{\Delta \alpha}{\alpha}
$$

Thus, one can introduce the variables $\Delta \alpha$ instead of $\Delta \rho$. Furthermore, from Eq. (3.12), one has 


$$
\Delta \alpha_{\text {Geff }}=\left\{\begin{array}{ll}
-\left(1-\alpha_{0}\right) \sum \Delta \alpha_{m} & \alpha_{G e f f}>\tilde{\alpha} \\
0 & \alpha_{G e f f}=\tilde{\alpha}
\end{array} .\right.
$$

Thus, $\Delta \alpha_{G}$ eff can be easily eliminated, and after some rearranging, one obtains the following system of equations for the iteration step $k+1$ :

$$
\begin{aligned}
& \mathrm{J}_{11} \Delta \alpha p+\mathrm{J}_{12} \Delta \alpha_{\mathrm{L} 1}+\mathrm{J}_{13} \Delta \alpha_{\mathrm{L} 2}=\Delta \mathrm{p}_{1}, \\
& \mathrm{~J}_{21} \Delta \alpha \mathrm{p}+\mathrm{J}_{22} \Delta \alpha \alpha_{\mathrm{L} 1}+\mathrm{J}_{23} \Delta \alpha_{\mathrm{L} 2}=\Delta \mathrm{p}_{2}, \text { and } \\
& \mathrm{J}_{31} \Delta \alpha \mathrm{p}+\mathrm{J}_{32} \Delta \alpha_{\mathrm{L} 1}+\mathrm{J}_{33} \Delta \alpha_{\mathrm{L} 2}=\Delta \mathrm{p} 3
\end{aligned}
$$

The off-diagonal matrix elements all have the same value and are equal to

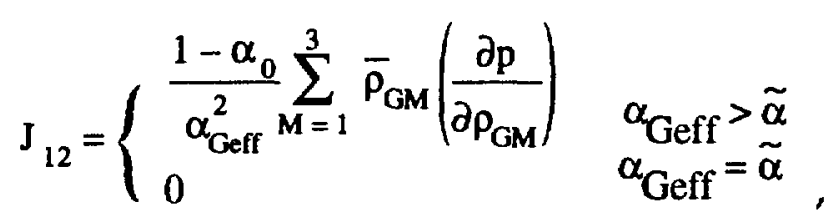

and

$$
\mathrm{J}_{13}=\mathrm{J}_{21}=\mathrm{J}_{31}=\mathrm{J}_{32}=\mathrm{J}_{12} .
$$

The diagonal matrix elements are

$$
\begin{aligned}
& \mathrm{J}_{11}=\mathrm{J}_{12}+\frac{\bar{\rho}_{\mathrm{p}}}{\alpha_{\mathrm{p}}^{2}}\left(\frac{\partial \mathrm{p}}{\partial \rho_{\mathrm{p}}}\right), \\
& \mathrm{J}_{22}=\mathrm{J}_{12}+\frac{\bar{\rho}_{\mathrm{L} 1}}{\alpha_{\mathrm{L} 1}^{2}}\left(\frac{\partial \mathrm{p}}{\partial \rho_{\mathrm{L} 1}}\right), \text { and } \\
& \mathrm{J}_{33}=\mathrm{J}_{12}+\frac{\bar{\rho}_{\mathrm{L} 2}}{\alpha_{\mathrm{L} 2}^{2}}\left(\frac{\partial \mathrm{p}}{\partial \rho_{\mathrm{L} 2}}\right) .
\end{aligned}
$$

The pressure differences $\Delta p$ are 


$$
\begin{aligned}
& \Delta p_{1}=p\left(\rho_{p}^{k}, T_{p}\right)-p_{G}, \\
& \Delta p_{2}=p\left(\rho_{L 1}^{k}, T_{L 1}\right)-p_{G},
\end{aligned}
$$

and

$$
\Delta p_{3}=p\left(\rho_{L 2}^{k}, T_{L 2}\right)-p_{G}
$$

where $\mathrm{PG}_{\mathrm{G}}$ is the gas pressure given by Eq. (3.4).

The above equations are solved by an explicitly programmed Gaussian elimination procedure. This yields the updated $\alpha_{m}$ for the condensed components. The relationships [Eqs. (3.10)-(3.12)] are then used to obtain the updated $\alpha_{\text {Geff }}$ and the microdensities for this iteration step.

If a component is missing, the routine sets the diagonal matrix element equal to one; the corresponding nondiagonal matrix elements and the pressure difference are set to zero. For example, if the particle component, described by Eq. (3.13), is missing, one has

$$
\mathrm{J}_{11}=1, \mathrm{~J}_{12}=\mathrm{J}_{13}=\Delta \mathrm{p}_{1}=0
$$

This leads automatically to a solution with

$$
\Delta \alpha p=0
$$

The initial guesses for the condensed component microdensities are usually those of the preceding call of the EOSPEQ routine. At the initialization of all the variables at time zero, the saturation values are used. Note, however, that the initial guesses must be larger than the liquid spinodal limit densities (Sec. 5). The other initial values are then obtained from Eqs. (3.10)-(3.12).

The convergence criterion is a pressure criterion. The iteration is continued until

$$
\left.\max || \Delta \mathrm{p}_{1}|,| \Delta \mathrm{p}_{2}|,| \Delta \mathrm{p}_{3} \mid\right\}<\varepsilon_{\mathrm{p}}
$$


The default value in the code is $\varepsilon_{\mathrm{p}}=10 \mathrm{~Pa}$, which is a tight criterion. Note that a relative pressure criterion (for example, $\max |\Delta \mathrm{p}| / \mathrm{p}<10^{-4}$ ) is preferable.

e. Single-Phase Mechanical Equilibrium Calculation. Single-phase flow cells can be identified from the converged two-phase mechanical equilibrium calculation. According to Sec. 3.a, cells that satisfy the following relationships are defined as single-phase cells.

$$
\alpha_{s}+\Sigma \alpha_{m}>1
$$

For these cells, EOSPEQ carries out a single-phase mechanical equilibrium calculation. The resulting microdensities of the compressed condensed components are called $\rho^{*}$, and the associated volume fractions are called $\alpha^{*}$. The conditions for the single-phase pressure equilibrium are then

$$
p\left(\rho^{*}, T_{P}\right)=p\left(\rho^{*}{ }_{1}, T_{L 1}\right)=p\left(\rho^{*}{ }_{2}, T_{L 2}\right)
$$

They must be supplemented by the relations

$$
\alpha_{m}^{*}=\bar{\rho}_{m} / \rho_{m}^{*}
$$

and by the volume balance condition

$$
\alpha_{s}+\Sigma \alpha_{m}^{*}=1
$$

The method of solution is, again, a Newton-Raphson iteration. At the iteration step $k+1$, one has

$$
\rho^{* k+1}=\rho^{* k}+\Delta \rho
$$

and

$$
p^{k+1}=p^{k}+\left(\frac{\partial p}{\partial \rho}\right) \Delta \rho
$$


One can again introduce the $\alpha$ 's as independent variables; from the volume balance [Eq. (3.16)], one has

$$
\Sigma \Delta \alpha_{m}^{*}=0
$$

Using Eq. (3.17) to eliminate $\Delta \alpha^{*} \mathrm{L2}$, one obtains the following equations in a similar way as the two-phase case:

$$
\begin{aligned}
& \mathrm{J}_{11} \Delta \alpha_{\mathrm{p}}^{*}+\mathrm{J}_{12} \Delta \alpha_{\mathrm{L} 1}^{*}=\Delta \mathrm{p}_{1}, \text { and } \\
& \mathrm{J}_{21} \Delta \alpha_{\mathrm{p}}^{*}+\mathrm{J}_{22} \Delta \alpha_{\mathrm{L} 1}^{*}=\Delta \mathrm{p}_{2} .
\end{aligned}
$$

Again, the two off-diagonal matrix elements are equal, that is,

$$
\mathrm{J}_{12}=\mathrm{J}_{21}=\frac{\bar{\rho}_{\mathrm{L} 2}}{\left(\alpha_{\mathrm{L} 2}^{*}\right)^{2}}\left(\frac{\partial p}{\partial \rho_{\mathrm{L} 2}^{*}}\right)
$$

The diagonal matrix elements are

$$
\begin{aligned}
& \mathrm{J}_{11}=\mathrm{J}_{12}+\frac{\bar{\rho}_{\mathrm{P}}}{\left(\alpha_{\mathrm{p}}^{*}\right)^{2}}\left(\frac{\partial \mathrm{p}}{\partial \rho_{\mathrm{p}}^{*}}\right), \text { and } \\
& \mathrm{J}_{22}=\mathrm{J}_{12}+\frac{\bar{\rho}_{\mathrm{L} 1}}{\left(\alpha_{\mathrm{L} 1}^{*}\right)^{2}}\left(\frac{\partial \mathrm{p}}{\partial \rho_{\mathrm{L} 1}^{*}}\right),
\end{aligned}
$$

and the pressure differences are

$$
\begin{aligned}
& \Delta p_{1}=p\left(\rho_{P}^{*}, T_{p}\right)-p\left(\rho_{L 2}^{*}, T_{L 2}\right), \text { and } \\
& \Delta p_{2}=p\left(\rho_{L 1}^{*}, T_{L 1}\right)-p\left(\rho_{L 2}^{*}, T_{L 2}\right) .
\end{aligned}
$$


The code solves the system [Eqs. (3.18-3.19)] for $\Delta \alpha^{*}$. The updated microdensities $\rho^{*}$ are then obtained from the relations [Eq. (3.15)]. The convergence criterion is analogous to Eq. (3.14).

The missing components are treated in a similar way as in the two-phase case. However, the logic becomes somewhat more complicated when liquid 2 is missing because its volume fraction change $\Delta \alpha^{*} L 2$ was chosen to be eliminated using Eq. (3.17). If it is missing, one of the other $\Delta \alpha^{* 1}$ s must be eliminated.

f. Partial Derivatives of the State Variables. The EOS interface with the SESAME 301 table is designed to give the pressure $p$, its partial derivatives with respect to the thermodynamic density $\rho$ and temperature $\mathrm{T}$ [i.e., $(\partial \mathrm{p} / \partial \rho)_{\mathrm{T}}$ and $\left.(\partial \mathrm{p} / \partial \rho)_{\rho}\right]$, the internal specific energy $\mathrm{e}$, and its partial derivatives $(\partial \mathrm{e} / \partial \rho)_{\mathrm{T}}$ and $(\partial e / \partial \rho)_{\rho}$, when the thermodynamic density $\rho$ and temperature $T$ are given; i.e., in both two-phase and single-phase pressure equilibrium calculations per iteration level $k$ at which the SESAME 301 table is interrogated with the input $\left(\rho^{k}, T\right)$, we obtain directly a set of output $\left[\mathrm{p}^{\mathrm{k}},(\partial \mathrm{p} / \partial \rho)_{T} \mathrm{k},(\partial \mathrm{p} / \partial \mathrm{T})_{\mathrm{p}} \mathrm{k}, \mathrm{e}^{\mathrm{k}},(\partial \mathrm{e} / \partial \rho)_{\mathrm{T}} \mathrm{k},(\partial \mathrm{e} / \partial \mathrm{T})_{\mathrm{p}} \mathrm{k}\right]$. Note that the temperature is constant during the mechanical equilibrium calculations. These partial derivatives after convergence are stored for later construction of the EOS function derivatives.

g. Treatment of the Liquid Spinodal Limit in the Mechanical Equilibrium Calculation (Routine EOSPEQ). As mentioned earlier, the components in an AFDM cell are usually not in phase equilibrium. If vapor density is less than equilibrium density, the liquid is in a superheated state (line BC in Fig. 3.4) and vaporizes. The amount of superheat depends on the heating rate and on the availability of nucleation sites. In experimental situations, the superheat is usually small, unless the system is very clean (no nucleation sites) or the heating rates are very large.

This last statement is somewhat vague. However, the only true physics limit for the superheat is at the spinodal line. This becomes obvious by looking at the statistical fluctuations of the number of molecules in a given volume $V$, which is determined by the equation

$$
\frac{\overline{(\Delta n)^{2}}}{n^{2}}=R T /\left(\frac{\partial p}{\partial \rho}\right)_{T} \text {. }
$$

At the spinodal line, one has $(\partial \mathrm{p} / \partial \rho)_{\mathrm{T}}=0$, and the density fluctuations become very large. This leads to the formation of vapor nuclei by a homogeneous 
nucleation mechanism, which is believed to occur in the time scale of 10-100 nanoseconds. 10 Note that the spinodal line was reached in exploding wire experiments at the University of Kiel. ${ }^{10}$ The heating rate in the experiments was some $10^{9} \mathrm{~K} / \mathrm{s}$, and it is believed that heating occurs at high pressure, and nearly isobaric pressure, up to the spinodal line, where a shock wave destroys the sample.

This is the physics background for the requirement in the AFDM EOS interface that a liquid not be allowed to expand below the spinodal density; this requirement is suggested by the fact that the derivative of the pressure with respect to density becomes negative below the spinodal density, and thus, this part of the Van-der-Waals loop (CD in Fig. 3.4) is thermodynamically unstable. Therefore, special treatment is necessary in the case where the AFDM models predict an expansion of one of the two liquids into this unstable region. This situation occurs when the cell pressure is lower than the pressure at the liquid spinodal point and can occur in AFDM when the cell pressure is lower than the pressure at the liquid spinodal point. This situation may be a numerical problem caused by a too-large timestep size, or it may be a result of a deficiency of the heat- and mass-transfer models, which may underestimate the vaporization rate during very rapid temperature transients. In the first case, the timestep problem can be avoided (as well as the related situation of a vapor spinodal limit treated in the next section) by choosing the timestep small enough so that the spinodal limits are not exceeded during the cycle (if necessary, by recalculating the cycle with repeatedly smaller timestep sizes).

It is known, and can be seen from Fig. 3.4, that the liquid spinodal pressure PSpl is negative over much of the temperature range of the liquid. In this case, equilibrium with the gas phase always exists. Thus, the approach to the liquid spinodal density $\rho$ Spl can occur only at rather high temperatures when the liquid spinodal pressure becomes positive (Fig. 3.4). For the Van-der-Waals equation, this situation occurs above the temperature $\mathrm{T}=0.844 \mathrm{~T}_{\mathrm{Crt}}$. With the EOS data presently used, this means that the fuel/steel/sodium temperature exceeds about $10000 / 8000 / 2000 \mathrm{~K}$, respectively. There are two principal ways to avoid the unstable region:

(1) The liquid is not allowed to expand below the liquid spinodal density. Thus, if the cell pressure drops below pspl, the liquid is treated in analogy to a missing component that does not take part in the pressure equilibrium. This liquid has density $\rho \mathrm{Spl}$. Unlike a true missing component, it occupies a finite volume fraction. 
(2) The liquid is allowed to expand below the density PSpl, assuming that the pressure is constant at value PSpl. As the liquid exparids, the other components in the cell will be compressed and reach equilibrium pressure PSpl.

Clearly, concept (1) is more compatible with physics because a liquid cannot expand to arbitrarily low density without evaporating. Thus, concept (1) is the one chosen for AFDM. The calculational treatment is not trivial because one has to avsid oscillations in and out of the spinodal limit; because one has to distinguish between three different kinds of liquid: normal, truly missing, and spinodal limit; and because the zero derivative $\partial \mathrm{p} / \partial \rho$ causes problems in the calculation of other derivatives.

One has to distinguish two different situations, which are characterized in Figs. 3.7 and 3.8. In the first situation, for the given amount of gas in the cell, the gas pressure at the smallest possible volume fraction $p\left[\alpha_{0}\left(1-\alpha_{L}\right)\right]$ is higher than $\mathrm{pSpl}$. Figure 3.7 shows the pressure of liquid $m$ (the one with the potential spinodal limit)

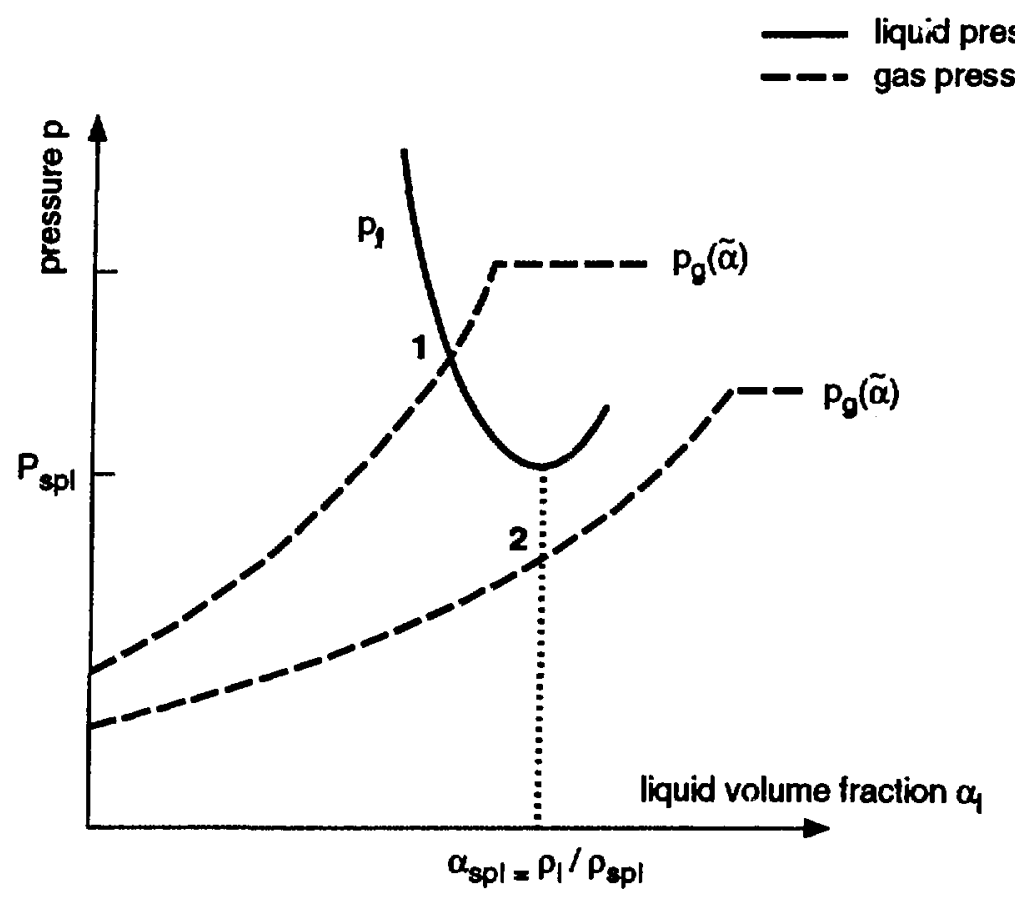

Fig. 3.7.

Two-phase pressure equilibrium for $\mathrm{pG}(\tilde{\alpha})>\mathrm{pSpl}$. 


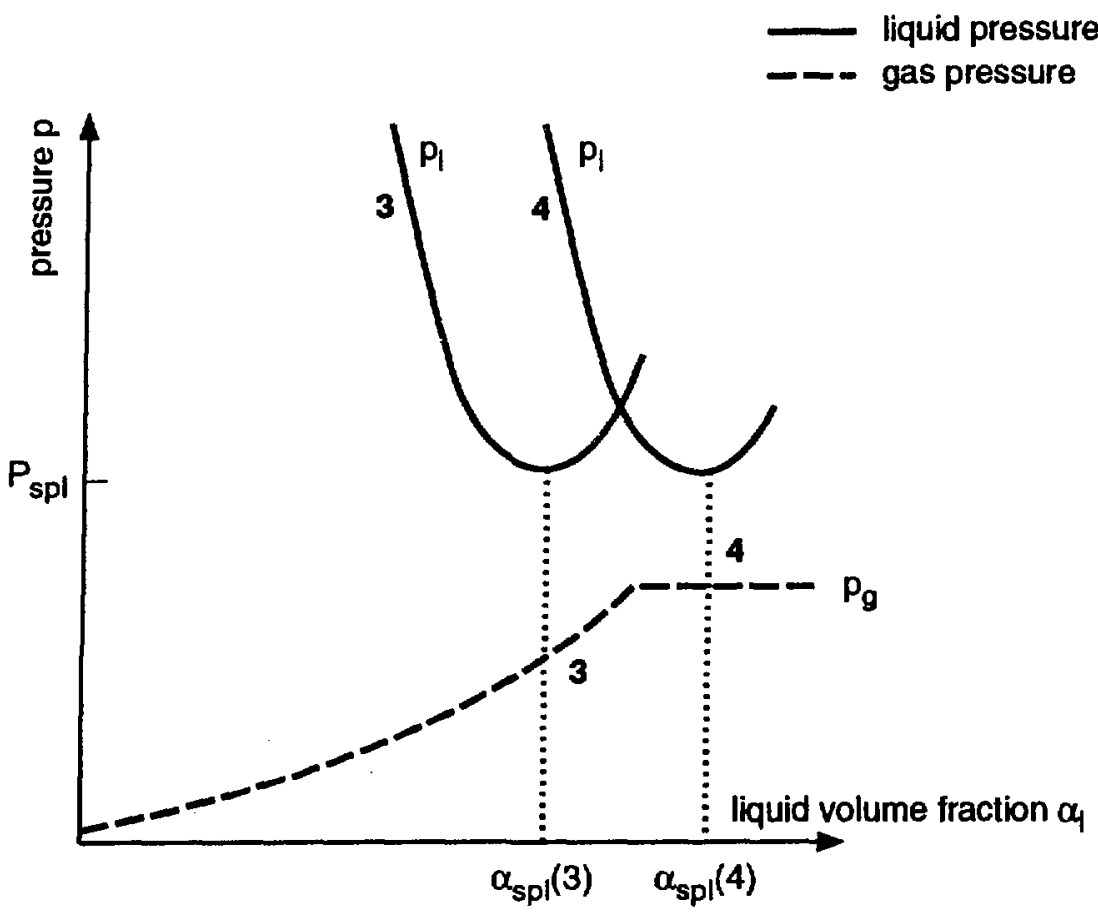

Fig. 3.8.

Two-phase pressure equilibrium for $\mathrm{pg}(\tilde{\alpha})<\mathrm{pspl}$.

and the gas pressure for two different gas contents in the cell. The variable $\alpha_{\mathrm{Lm}}$ determines the densities for given temperatures and $\bar{\rho} s$, and therefore, the pressures. For simplicity, it is assumed that the condensed components $m^{\prime} \neq m$ are in equilibrium with the vapor phase.

Case 1 The gas content is high enough that pressure equilibrium exists for a certain value of $\alpha_{\mathrm{Lm}}$, which must be found by the mechanical equilibrium iteration ("normal" case). The density of the liquid does not reach the spinodal limit value. In Fig. 3.\%, this state corresponds to point 1.

Case 2 The low gas content does not allow for pressure equilibrium. In this case, the liquid density is fixed at the spinodal limit value (thus, $\alpha_{\mathrm{Lm}}=\bar{\rho}_{\mathrm{Lm}}$ ' $\left.\rho_{S p l, m}\right)$, and the gas pressure is lower than pspl. In Fig. 3.7, this state corresponds to point 2.

Note that Cases 1 and 2 are always two-phase states. There is enough gas in the cell to exclude single-phase situations. 
A different situation occurs when the gas pressure at the smallest allowable volume fraction $\tilde{\alpha}=\alpha_{0}\left(1-\alpha_{s}\right)$ is lower than the spinodal limit pressure. Thus,

$$
\mathrm{p}_{\mathrm{G}}(\tilde{\alpha})<\mathrm{p}_{\mathrm{Spl}, \mathrm{m}} .
$$

The pressures in this situation are presented in Fig. 3.8, again as a function of $\alpha_{\mathrm{Lm}}$. Curves 3 and 4 correspond to different amounts of liquid m present in the cell, while the gas content is the same in both cases. Note that a two-phase equilibrium between the gas and liquid $m$ does not exist because above a certain value of $\alpha_{\mathrm{Lm}}$, the gas pressure is constant and the liquid pressure formally increases in the unstable branch of the Van-der-Waals loop.

In Case 3, the liquid expands until the spinodal limit density and the corresponding gas (or cell) pressure is lower than pspl. The cell is in a two-phase state, which corresponds to point 3 in Fig. 3.8.

Case 4 corresponds to a single-phase case. In the two-phase iteration, the liquid reaches the spinodal limit density. At this point (Point 4 in Fig. 3.8), the iteration must be stopped because both the liquid and the gas pressures have zero derivatives.

After the two-phase iteration, a single-phase iteration must be carried out in Case 4. Figure 3.9 shows the pressures as functions of the "compressed" $\alpha^{*} \mathrm{Lm}$. There is the pressure of liquid $m$ (Curve 4) and also the pressure of an additional condensed material $\mathrm{m}^{\prime}$; Curves $4 \mathrm{a}$ and $4 \mathrm{~b}$ correspond to different amourts of material $\mathrm{m}^{\prime}$ and lead to two different subcases, $4 \mathrm{a}$ and $4 \mathrm{~b}$. In Case $4 \mathrm{a}$, the single-phase compression $\alpha m^{\prime}-\alpha^{*} m^{\prime}$ of material $m^{\prime}$ is so strong that its pressure increases above the PSpl,m of liquid $m$. Thus, a normal single-phase equilibrium exists (with $\rho_{\mathrm{Lm}}>\rho$ Spl,m), although the cell was a "spinodal limit case" in the preceding twophase iteration. Note, however, that this case occurs only in a very narrow window of macroscopic densities of the two materials $\mathrm{m}$ and $\mathrm{m}$ '.

The more frequent subcase is Case $4 b$, where the amount of $m$ ' is so low that its pressure at the volume fraction $\alpha^{*} \mathrm{Lm}$ of $\mathrm{m}$ is below the spinodal limit $\mathrm{pSpl,m}$. In this case, a "spinodal limit" occurs also in the single-phase iteration. In the routine EOSPEQ, the following steps are introduced to treat spinodal limit cases:

- During initialization, the routine SPINDM is called for the two liquids, $\mathrm{m}=3$ and $\mathrm{m}=4$. The spinodal values $\rho_{\mathrm{Spl}, \mathrm{m}}, \mathrm{pSpl,m}$, and $\alpha_{\mathrm{SpL}, \mathrm{m}}=\bar{\rho}_{\mathrm{Lm}}$ ' 


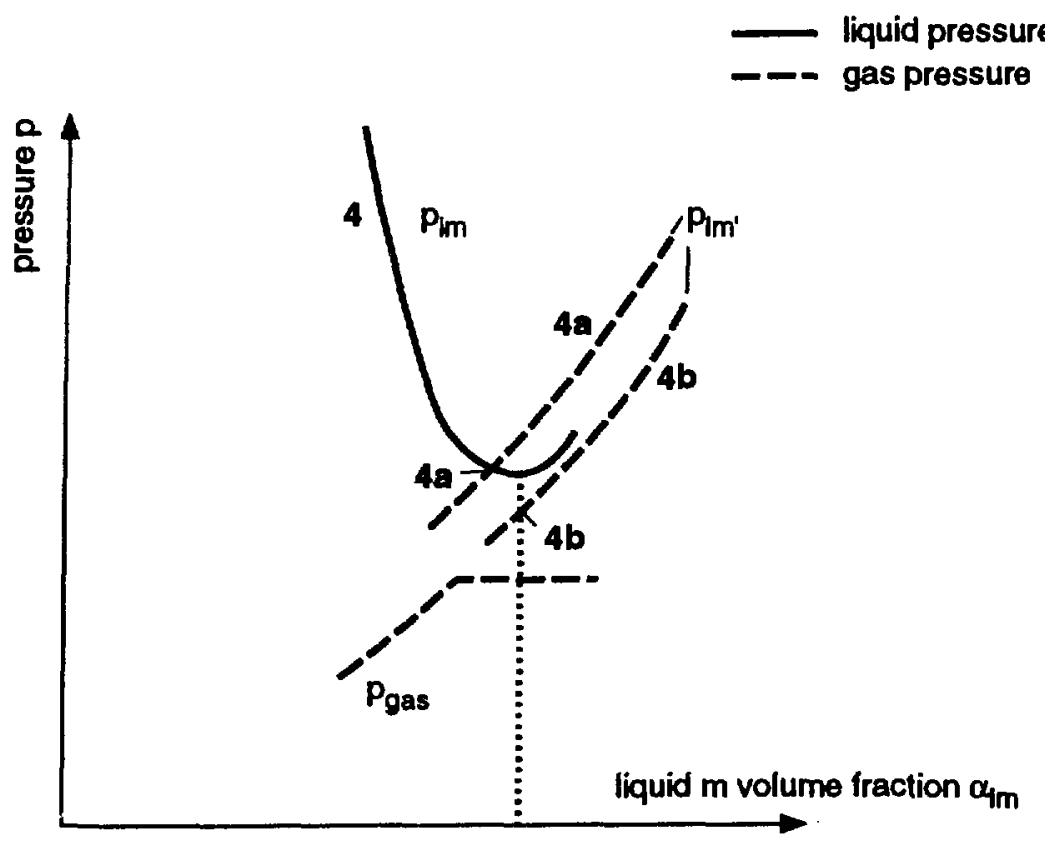

Fig. 3.9.

Single-phase pressure equilibrium.

$\rho$ Spl,m are defined. Note that the initial values for $\rho_{\mathrm{Lm}}$ must be a few percent larger than $\rho \mathrm{Spl}, \mathrm{m}$. Otherwise, the iteration starts with a zero tangent.

- If, during the iteration, $\alpha$ exceeds the limit $\alpha_{m}+\Delta \alpha_{m}>\alpha_{S p l, m}$, then $\alpha_{\mathrm{Lm}}$ is replaced by $\alpha_{S p l, m}$, and the "component present" logical variable $\operatorname{LER}(\mathrm{m}-1)$ is set to 0 (missing component).

- If one tries to use a pressure criterion $p_{G}<$ pSpl,m to set LER(m-1) to 0 , then there are oscillations in two-phase iteration. Therefore, the " $\alpha$-criterion" was chosen. This criterion is irreversible in the sense that once LER is set to 0 , it cannot go back to 1 during the two-phase iteration.

- If the spinodal limit occurs in a single-phase cell, one has $\alpha_{\text {Geff }}=\tilde{\alpha}$ and pG $<$ pSpl,m. In this case (Case 4), the two-phase iteration does not converge, even when $\alpha_{\mathrm{Lm}}$ is set to its spinodal limit value (see Fig. 3.8). It is therefore necessary to discontinue the iteration by putting $W \mathrm{pm}_{\mathrm{m}}=0$. It is always true that pSpl,m $>$ pG but it is not necessarily true that $\alpha_{m}>\alpha_{\text {Spl,ml }}$. Therefore, the "pressure criterion" is used together with $\alpha_{\mathrm{Geff}} \leq \tilde{\alpha}$ to put $\Delta \mathrm{p}_{\mathrm{m}}=0$. 
- If a cell is single phase, the component present variable is set to 1 at the beginning of the iteration unless it is truly a missing component with low density.

- The procedure during the single-phase iteration is much the same as with the two-phase iteration. However, in this case, the volume balance relation

$$
\Sigma \alpha_{m}^{*}=1 \text { or } \Sigma \Delta \alpha_{m}^{*}=0
$$

must be preserved (the $\alpha^{*}$ 's are the single-phase volume fractions).

Thus, when $\alpha_{m}^{*}+\Delta \alpha_{m}^{*}$ is replaced by $\alpha_{\mathrm{Spl}, \mathrm{m}}$, the difference between the

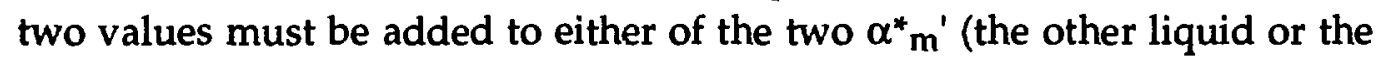
particle field, whichever is present).

\section{h. Treatment of the Vapor Spinodal Limit in the Mechanical Equilibrium} Calculation (Routine EOSPEQ). In an EOS call, the macroscopic density of one the vapor components may be so large that an equilibrium with a density below the vapor spinodal limit does not exist. In other words, the vapor may be predicted to compress beyond its spinodal density. In the EOS interface, this case is handled as follows (Fig. 3.10):

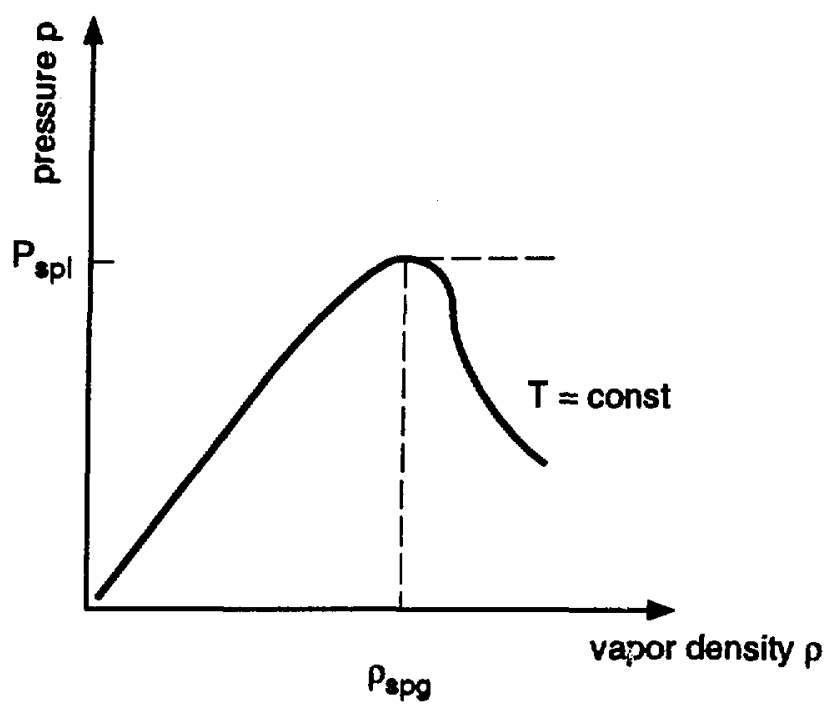

Fig. 3.10.

Isotherm in the gas range and vapor spinodal limit. 
The isotherm $p(\rho)$ is continued beyond $\rho$ Spg with a horizontal tangent. This way, the property of the vapor being a compressible component is preserved; only the compression occurs at constant pressure. Thus, in EOSPEQ, if $\rho>\rho S p g$, put $\mathrm{p}=\mathrm{pSpg}$ (spinodal pressure),$\partial \mathrm{p} / \partial \rho=0$, and $\partial \mathrm{p} / \partial \mathrm{T}=(\partial \mathrm{p} / \partial \mathrm{T})_{\mathrm{s}}$, which is the derivative along the spinodal line. Thus, the function $p(p, T)$ is continuous, though the partial derivatives are discontinuous at $\rho=\rho$ Spg. These partials are then returned for calculation of all the partial derivatives needed for the EOS interface.

To extend the specific internal energy e in a thermodynamically consistent way, one should use the thermodynamic equation

$$
\rho^{2}\left(\frac{\partial e}{\partial \rho}\right)_{T}=-T\left(\frac{\partial p}{\partial T}\right)_{\rho}+p .
$$

Because the right-hand side is independent of density for $\rho>\rho$ Spg,

$$
e(\rho, T)=e_{S_{P B}}(T)-\left(\frac{1}{\rho_{S p g}}-\frac{1}{\rho}\right)\left[T\left(\frac{\partial p}{\partial T}\right)_{\rho}-p_{S_{P B}}\right]
$$

Then,

$$
\left(\frac{\partial \mathrm{e}}{\partial \rho}\right)_{T}=-\frac{1}{\rho^{2}}\left[\mathrm{~T}\left(\frac{\partial p}{\partial T}\right)_{\rho}-p\right]
$$

To find $\partial \mathrm{e} / \partial \mathrm{T}$, one can assume that the second term in $\mathrm{e}(\rho, \mathrm{T})$ is small compared to the first one and can be approximately written as

$$
\left(\frac{\partial \mathrm{e}}{\partial T}\right)_{\rho} \approx\left(\frac{\partial \mathrm{e}_{\mathrm{Spg}}}{\partial \mathrm{T}}\right)
$$

The quantities needed from a call of SPINDOM are

$$
\text { PSpg, dpspg } / \text { dT, } \rho_{\text {Spg }} \text {. }
$$


The vapor spinodal limit is not as complicated to handle in EOSPEQ as the liquid spinodal limit because a straightforward extension of the isotherms on the vapor side is used. An alternative would be to modify the SESAME tables to include this extension, i.e., tabulate the constant pressure up to, say, the critical density. Both the pressure and internal energy tables would have to be modified. With tables conditioned in this way, there would be no need for special treatment of the vapor spinodal limit in the EOSPEQ routine.

\section{Saturation Properties}

a. Saturation Temperature. The components in a cell are generally not in saturated states. Consequently, saturation properties are not needed for the mechanical equilibrium calculation. They are, however, needed in the following contexts:

- Mainly, because they are used in the heat- and mass-transfer models, the models assume that heat flow between a liquid and its vapor occurs via the interfacial area between the two phases. The interfaces are postulated to be in saturated states that are determined by the vapor partial pressure. The pressure defines the saturation temperature and thus, also saturation densities and enthalpies on the liquid and vapor side. These properties z.re obtained by interrogating the SESAME 401 table. Note that this saturation state is fictitious and that the "liquid saturation" properties have nothing to do with the actual state of the liquid.

- For the nucleation model to describe vapor bubble nucleation sites in a liquid, it uses a saturation state that is determined by the liquid saturation temperature (that is, liquid temperature minus superheat).

- Additionally, the missing (liquid or particle) components that do not take part in the mechanical equilibrium are assumed to be in saturated states.

b. Other Saturation Properties. More saturation properties are required by the AFDM algorithm, for example,

- condensed liquid density: $\rho$ Con, $M(m)$ for $m=3,4$;

- saturated vapor density: $\rho V a p, M(m)$ for $m=5,6$;

- saturation pressure: $\mathrm{PSat}, \mathrm{M}(\mathrm{m})$ for $m=3$ through 6

- condensated liquid specific internal energy: ${ }^{2}$ Con, $M(m)$ for $m=3,4$; and 
- saturated vapor specific internal energy: evap, $M(m)$ for $m=5,6$, which are directly available from the SESAME 401 table with the temperatures specified. From the properties, the following components can be derived:

specific internal heat of vaporization is then

- elg,M = eVap,M - eCon, $M$ for $M=1,2$,

and the condensated liquid and saturated vapor specific enthalpies are

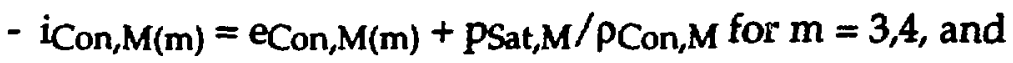

- $\mathrm{i} \operatorname{Vap}, M(m)=\operatorname{eVap}, M(m)+$ PSat $_{,} M / \rho_{\text {Vap, }} M$ for $m=5,6$ and $M=1,2$.

the specific heat of vaporization enthalpy follows to

- $h_{\text {lg, }, M}=i$ Vap, $M-i$ Con, $M$ for $M=1,2$.

Partial derivatives of these properties are discussed in Sec. 6 and in Chap. II.C.

5. Temperature Inverse Calculations

a. Basic Equations. The AFDM algorithm requires some inverted EOS evaluations. These temperature evaluations give the macrodensity and internal energy. Inverted EOS evaluations are therefore needed for the five energy components $S, P$, L1, L2, and G. Obtaining the temperatures requires an iterative procedure because the internal energy, density, and temperatures are related by nonlinear equations.

For $m^{\text {th }}$ energy component temperature, given the internal energy $e_{m}{ }^{0}$, the equation to be solved is

$$
e_{m}^{0}-e_{m}\left(\rho_{m}, T_{m}\right)=0
$$

Here, $e_{m}$ is the internal energy of the $m$ component with thermodynamic density $\rho_{m}$ and temperature $T_{m}$. Especially for the vapor phase, Eq. (3.20) can be written, using Eq. (3.5) with e $\mathrm{G}^{0}$ given, as

$$
\mathrm{e}_{G}^{0}-\sum_{M=1}^{3} x_{G M} e_{G M}\left(P_{G M}, T_{G}\right)=0
$$

where

$$
\mathrm{X}_{\mathrm{GM}}=\frac{\bar{\rho}_{\mathrm{GM}}}{\bar{\rho}_{\mathrm{G} 1}+\bar{\rho}_{\mathrm{G} 2}+\bar{\rho}_{\mathrm{G} 3}} .
$$


The mechanical equilibrium condition requires

$$
\sum_{M=1}^{3} \rho\left(\rho_{G M}, T_{G}\right)=p\left(\rho_{p}, T_{p}\right)=p\left(\rho_{L 1}, T_{L 1}\right)=p\left(\rho_{L 2}, T_{L 2}\right)
$$

and the volume balance

$$
\alpha_{\text {Geff }}=\max \left[\alpha_{0}\left(1-\alpha_{s}\right), 1-\alpha_{s}-\left(1-\alpha_{0}\right) \sum_{m} \alpha_{m}\right]
$$

must be satisfied.

If the variables $\mathrm{p}$ are replaced by the $\alpha$ 's through the relations

$$
\rho=\bar{\rho} / \alpha,
$$

then one has a system of eight equations for the four temperatures, plus the four volume fractions.

The simultaneous solution of these equations by a Newton-Raphson method seems to be prohibitive. Two different methods of solution are feasible:

- iterating back and forth between the "energy equations" (3.20/3.21) and the "pressure equilibrium equations" (3.23/3.24). Thus, guess values of $\rho$ are used to solve the energy equations for the temperatures. These temperatures are then used to solve the pressure equilibrium equations for improved density values, as described in Sec. 3.e. Such a procedure converges, but it involves a double iteration.

- One can observe that the number of equations for the iteration can be reduced. Then a procedure much like the one used for pressure equilibrium (Sec. 3.8) can be applied. This method, which involves only one set of iterations, is used in the code. It will be next.

For this simplification, one must formally introduce the temperatures as functions of the densities $\rho_{m}$ and the internal energies $e_{m}$ as independent variables. Then the pressures are given by

$$
p_{m}=p_{m}\left[\rho_{m}, T_{m}\left(e_{m} \rho_{m}\right)\right]
$$


for the condensed materials and by

$$
\mathrm{p}_{\mathrm{G}}=\sum_{M=1}^{3} \mathrm{p}\left[\mathrm{p}_{\mathrm{GM}}, \mathrm{T}_{\mathrm{G}}\left(\mathrm{e}_{\mathrm{G}}, \rho_{\mathrm{GM}}\right)\right]
$$

for the vapor mixture. Then the set of Eqs. (3.23 and 3.24) must be solved. The Newton-Raphson procedure requires a linearization, which reads for the iterative step $k+1$ as

$$
p^{(k+1)}=p^{(k)}+\Delta p
$$

For the condensed materials, $\Delta p$ can then be expressed by the variables $\Delta \rho_{m}$ and $\Delta e_{m}$.

$$
\Delta p_{m}=\left[\left(\frac{\partial p}{\partial \rho_{m}}\right)_{T_{m}}+\left(\frac{\partial p}{\partial T_{m}}\right)_{\rho_{m}}\left(\frac{\partial T_{m}}{\partial \rho_{m}}\right)_{c_{m}}\right] \Delta \rho_{m}+\left(\frac{\partial p}{\partial T_{m}}\right)_{\rho_{m}}\left(\frac{\partial T_{m}}{\partial e_{m}}\right)_{\rho_{m}} \Delta e_{m}
$$

From the thermodynamic equation

$$
\mathrm{de}=\left(\frac{\partial \mathrm{e}}{\partial \mathrm{T}}\right)_{\rho} \mathrm{dT}+\left(\frac{\partial \mathrm{e}}{\partial \rho}\right)_{\mathrm{T}} \mathrm{d} \rho
$$

one finds that $\partial T_{m} / \partial \rho_{m}$ can be written

$$
\left(\frac{\partial T_{m}}{\partial \rho_{m}}\right)_{e_{m}}=-\left(\frac{\partial e_{m}}{\partial \rho_{m}}\right)_{T_{m}}\left(\frac{\partial e_{m}}{\partial T_{m}}\right)_{\rho_{m}}^{-1}
$$

In addition, it is useful to introduce $\Delta \alpha_{\mathrm{m}}$ as an independent variable, instead of $\Delta p_{\mathrm{m}}$.

$$
\Delta \rho_{\mathrm{m}}=-\frac{\bar{\rho}_{\mathrm{m}}}{\alpha_{\mathrm{m}}^{2}} \Delta \alpha_{\mathrm{m}}
$$

Then Eq. (3.25) reads 


$$
\begin{aligned}
\Delta p_{m} & =\left[\left(\frac{\partial p}{\partial \rho_{m}}\right)_{T_{m}}-\left(\frac{\partial p}{\partial T_{m}}\right)_{\rho_{m}} \frac{\left(\partial e_{m} / \partial \rho_{m}\right)_{T_{m}}}{\left(\partial e_{m} / \partial T_{m}\right)_{\rho_{m}}} \frac{\bar{\rho}_{m}}{\alpha_{m}^{2}} \Delta \alpha_{m}+\left(\frac{\partial p}{\partial T_{m}}\right)_{\rho_{m}}\left(\frac{\partial T_{m}}{\partial e_{m}}\right)_{\rho_{m}} \Delta e_{m}\right. \\
& =-A_{m} \Delta \alpha_{m}+B_{m} \Delta e_{m} .
\end{aligned}
$$

Equation (3.26) holds for the condensed materials ( $m=P, L 1, L 2)$. The corresponding equation for the vapor composition will now be derived as:

$$
\begin{aligned}
& \mathrm{p}_{\mathrm{G}}=\sum_{M=1}^{3} \mathrm{p}\left(\rho_{\mathrm{GM}}, \mathrm{T}_{\mathrm{G}}\right) \text {, and } \\
& \Delta \mathrm{p}_{\mathrm{G}}=\sum_{M=1}^{3}\left(\frac{\partial \mathrm{p}}{\partial \rho_{\mathrm{GM}}}\right)_{\mathrm{T}_{G}} \Delta \rho_{\mathrm{GM}}+\sum_{M=1}^{3}\left(\frac{\partial \mathrm{p}}{\partial \mathrm{T}_{\mathrm{G}}}\right)_{\mathrm{P}_{\mathrm{OM}}} \Delta \mathrm{T}_{\mathrm{GM}} .
\end{aligned}
$$

Because $\alpha_{G}$ is the same for all gas components, the relative density changes are also the same:

$$
\frac{\Delta \rho_{\mathrm{GM}}}{\rho_{\mathrm{GM}}}=\Delta \varepsilon_{\mathrm{G}},
$$

where $\Delta \varepsilon_{G}$ is independent of $M$.

To relate $\Delta T_{G}$ to the change in internal energy, the definition of eG [from Eq. (3.21)] is lised:

$$
e_{G}=\sum_{M=1}^{3} x_{G M} e_{G M}\left(\rho_{G M}, T_{G}\right)
$$

Differentiating this equation,

$$
\begin{aligned}
& \Delta e_{G}=\left[\sum_{M=1}^{3} X_{G M}\left(\frac{\partial e_{G M}}{\partial \rho_{G M}}\right)_{T_{G}} \rho_{G M}\right] \Delta \varepsilon_{G}+\left[\sum_{M=1}^{3} X_{G M}\left(\frac{\partial e_{G M}}{\partial T_{G}}\right)_{P_{G M}}\right] \Delta T_{G} \text { or } \\
& \Delta e_{G}=e_{g \rho} \Delta \varepsilon_{G}+e_{g T} \Delta T_{G} .
\end{aligned}
$$


Solving for $\Delta \mathrm{T}_{\mathrm{G}}$ gives

$$
\Delta \mathrm{T}_{\mathrm{G}}=\frac{\Delta \mathrm{e}_{\mathrm{G}}-\mathrm{e}_{\mathrm{g} \rho} \Delta \varepsilon_{\mathrm{G}}}{\mathrm{e}_{\mathrm{gT}}}
$$

Inserting into Eq. (3.27), one obtains

$$
\Delta \mathrm{p}_{G}=\left[\mathrm{p}_{g p}-\mathrm{p}_{\mathrm{gT}} \frac{\mathrm{e}_{g \rho}}{\mathrm{e}_{\mathrm{gT}}}\right] \Delta \varepsilon_{\mathrm{G}}+\frac{\mathrm{p}_{g T}}{\mathrm{e}_{\mathrm{gT}}} \Delta \mathrm{e}_{\mathrm{G}},
$$

where the definitions

$$
\mathrm{p}_{\mathrm{g \rho}}=\sum_{\mathrm{M}=1}^{3}\left(\frac{\partial \mathrm{p}_{\mathrm{GM}}}{\partial \rho_{\mathrm{GM}}}\right)_{\mathrm{T}_{\mathrm{G}}} \rho_{\mathrm{GM}}
$$

and

$$
\mathrm{p}_{\mathrm{gT}}=\sum_{M=1}^{3}\left(\frac{\partial \mathrm{p}_{\mathrm{GM}}}{\partial \mathrm{T}_{\mathrm{G}}}\right)_{\mathrm{P}_{\mathrm{CM}}}
$$

were used. If $\alpha_{G \text { eff }}$ is introduced as a variable, then Eq. (3.28) can be written as

$$
\Delta p_{G}=-\left[p_{g p}-p_{G T} \frac{e_{g \rho}}{e_{g T}}\right] \frac{\Delta \alpha_{G e f f}}{\alpha_{G e f f}}+\frac{p_{g T}}{e_{g T}} \Delta e_{G}
$$

This equation is the equivalent for the vapor of Eq. (3.24) for the condensed materials.

The iterative procedure is then completely analogous to the procedure described in Sec. 3.e. It is based on the condition ( $k=$ iteration step):

$$
\mathrm{p}_{\mathrm{G}}^{(\mathrm{k})}+\Delta \mathrm{p}_{\mathrm{G}}=\mathrm{p}_{\mathrm{m}}^{(\mathrm{k})}+\Delta \mathrm{p}_{\mathrm{m}}
$$

b. Description of the Temperature Inversion Calculation. The temperature inversion method involves a mechanical equilibrium calculation, which is similar 
to the one described in Secs. 3.e and 3.f, but where the input variables are now internal energy and density. In the first step, the two-phase mechanical equilibrium calculation is carried out. Then the single-phase cells are gathered, and the singlephase equilibrium calculation is performed. This is done in the routine EOST.

In the two-phase equilibrium calculation, Eq. (3.30) is solved by a NewtonRaphson iterative procedure. In the linear approximation, the $\Delta \mathrm{p}$ 's are given by Eqs. (3.26) and (3.29). Again, $\Delta \alpha_{\text {Geff }}$ is eliminated using Eq. (3.24), so that there are three unknowns, $\Delta \alpha_{m}(m=P, L 1, L 2)$. Note that the $\Delta e^{\prime}$ s are known because the required values for the internal energies, $e^{0} \mathrm{~m}$ and $\mathrm{e}^{\circ} \mathrm{G}$ are given. Thus,

$$
\Delta e_{m}=e_{m}^{o}-e_{m}^{(k)}
$$

The system of three equations,

$$
\Delta p_{G}-\Delta p_{m}=p_{m}^{(k)}-p_{G}^{(k)},
$$

can be written

$$
\begin{aligned}
& \mathrm{J}_{11} \Delta \alpha_{\mathrm{P}}+\mathrm{J}_{12} \Delta \alpha_{\mathrm{L} 1}+\mathrm{J}_{13} \Delta \alpha_{\mathrm{L} 2}=(\mathrm{pP}-\mathrm{pG})_{\text {eff }} \\
& \mathrm{J}_{21} \Delta \alpha \mathrm{P}+\mathrm{J}_{22} \Delta \alpha_{\mathrm{L} 1}+\mathrm{J}_{23} \Delta \alpha_{\mathrm{L} 2}=(\mathrm{p} \mathrm{L} 1-\mathrm{pG})_{\text {eff }}, \text { and } \\
& \mathrm{J}_{31} \Delta \alpha \mathrm{P}+\mathrm{J}_{32} \Delta \alpha_{\mathrm{L} 1}+\mathrm{J}_{33} \Delta \alpha_{\mathrm{L} 2}=(\mathrm{pL} 2-\mathrm{pG})_{\text {eff }} .
\end{aligned}
$$

The nondiagonal matrix elements $\mathrm{J}_{\mathrm{mn}}$ are all equal.

$$
J_{m n}=\frac{C_{o}}{\left(\alpha_{G e f f}\right)^{2}}\left[p_{g \rho}-p_{g T} \frac{e_{g \rho}}{e_{g T}}\right] \text {, }
$$

where $C_{o}=\left(1-\alpha_{0}\right) H<\alpha_{G e f f}, \tilde{\alpha}>$ with $H<\alpha_{G e f f}, \tilde{\alpha}>=\left\{\begin{array}{l}0 \text { for } \alpha_{G e t r}<\tilde{\alpha} \\ 1 \text { for } \alpha_{\text {Get }}>\tilde{\alpha}\end{array}\right.$.

The diagonal elements are

$$
\begin{aligned}
& \mathrm{J}_{11}=\mathrm{J}_{12}+A_{P}, \\
& \mathrm{~J}_{22}=\mathrm{J}_{12}+A_{L 1} \text {, and } \\
& \mathrm{J}_{33}=\mathrm{J}_{12}+A_{\mathrm{L} 2},
\end{aligned}
$$


where $A_{m}$ is defined by Eq. (3.26). The "effective" pressure differences on the righthand side of Eq. (3.31) are

$$
\left(p_{m}-p_{G}\right)_{f f}=p\left(\rho_{m}, T_{m}\right)^{(k)}-p_{G}^{(k)}+\left(\frac{\partial p_{m}}{\partial T_{m}}\right)_{\rho_{m}} /\left(\frac{\partial e_{m}}{\partial T_{m}}\right)_{\rho_{m}}\left(e_{m}^{o}-e_{m}^{(k)}\right)-\frac{p_{g T}}{e_{g T}}\left(e_{G}^{o}-e_{G}^{(k)}\right) .
$$

The solution of Eq. (3.31) gives the changes $\Delta \alpha$. Besides, one needs to update the temperatures in each iteration step. As the temperatures are functions of internal energy and density (or volume fraction $\alpha$ ), one has the equation

$$
\Delta T_{m}=\frac{\Delta e_{m}}{\left(\partial e_{m} / \partial T_{m b}\right)}-\left(\frac{\partial T_{m}}{\partial \rho_{m}}\right) \frac{\bar{\rho}_{m}}{\alpha_{m}^{2}} \Delta \alpha_{m}
$$

for the condensed materials, and

$$
\Delta T_{G}=\frac{\Delta e_{G}}{e_{g T}}+\frac{e_{g \rho}}{e_{g T}} \frac{\Delta \alpha_{G e f f}}{\left(\alpha_{G e f f}\right)^{2}}
$$

for the gas mixture.

The iteration is continued until the convergence criterion

$$
\left|\left(p_{m}-p_{G}\right)_{e f f}\right|<\varepsilon_{p}
$$

is fulfilled for all three condensed materials.

After this calculation, one finds the single-phase cells from the criterion

$$
\alpha_{o}\left(1-\alpha_{s}\right)>1-\alpha_{s}-\left(1-\alpha_{o}\right) \Sigma \alpha_{m}
$$

For these cells, the single-phase mechanical equilibrium calculation is carried out. The equations (for the case where L2 is present in the cell) for the "compressed" $a^{*}$ values are 


$$
\begin{aligned}
& \mathrm{J}_{11} \Delta \alpha^{*} \mathrm{P}+\mathrm{J}_{12} \Delta \alpha^{*} \mathrm{~L} 1=\left[\mathrm{pP}(\mathrm{k})-\mathrm{PL}_{2}{ }^{(\mathrm{k})}\right]_{\mathrm{eff}} \text {, and } \\
& \mathrm{J}_{21} \Delta \alpha^{*} \mathrm{P}+\mathrm{J}_{22} \Delta \alpha^{*} \mathrm{~L} 1=\left[\mathrm{PL1}^{(\mathrm{k})}-\mathrm{PL2}^{(\mathrm{k})}\right]_{\mathrm{eff}},
\end{aligned}
$$

where the matrix elements are

$$
\begin{aligned}
& \mathrm{J}_{12}=\mathrm{J}_{21}=A_{L 2}, \\
& J_{11}=J_{12}=A_{P}, \text { and } \\
& J_{22}=J_{12}=A_{L 1} .
\end{aligned}
$$

and the "effective" pressure differences on the right-hand side are

$$
\begin{aligned}
& \left(\mathrm{p}_{\mathrm{P}}^{(\mathrm{k})}-\mathrm{p}_{\mathrm{L2}}^{(\mathrm{k})}\right)_{\text {eff }}=\mathrm{p}\left(\mathrm{\rho}_{\mathrm{P}}, \mathrm{T}_{\mathrm{P}}\right)^{(\mathrm{k})}-\mathrm{p}\left(\mathrm{\rho}_{\mathrm{L} 2}, \mathrm{~T}_{\mathrm{L} 2}\right)^{(\mathrm{k})} \\
& +\frac{\partial p / \partial T_{p}}{\partial e_{p} / \partial T_{p}}\left(e_{p}^{o}-e_{p}^{(k)}\right)-\frac{\partial p / \partial T_{L 2}}{\partial e_{L 2} / \partial T_{L 2}}\left(e_{L 2}^{o}-e_{L 2}^{(k)}\right) \text {, and } \\
& \left(\mathrm{P}_{\mathrm{L} 1}^{(\mathrm{k})}-\mathrm{P}_{\mathrm{L} 2}^{(\mathrm{k})}\right)_{\text {eff }}=\mathrm{p}\left(\rho_{\mathrm{L} 1}, \mathrm{~T}_{\mathrm{L} 1}\right)^{(\mathrm{k})}-\mathrm{p}\left(\rho_{\mathrm{L} 2}, \mathrm{~T}_{\mathrm{L} 2}\right)^{(\mathrm{k})} \\
& +\frac{\partial \mathrm{p} / \partial \mathrm{T}_{\mathrm{L} 1}}{\partial \mathrm{e}_{\mathrm{L} 1} / \partial \mathrm{T}_{\mathrm{L} 1}}\left(\mathrm{e}_{\mathrm{L} 1}^{\mathrm{o}}-\mathrm{e}_{\mathrm{L} 1}^{(\mathrm{k})}\right)-\frac{\partial \mathrm{p} / \partial \mathrm{T}_{\mathrm{L} 2}}{\partial \mathrm{e}_{\mathrm{L} 2} / \partial \mathrm{T}_{\mathrm{L} 2}}\left(\mathrm{e}_{\mathrm{L} 2}^{\mathrm{o}}-\mathrm{e}_{\mathrm{L} 2}^{(\mathrm{k})}\right)
\end{aligned}
$$

After solving Eq. (3.34), one obtains the temperatures $\Delta T_{m}$, as in the twophase case from Eq. (3.32). The convergence criterion in this case is

$$
\left|\left(\mathrm{p}_{\mathrm{m}}-\mathrm{p}_{\mathrm{L} 2}\right)_{\mathrm{eff}}\right|<\varepsilon_{\mathrm{p}}
$$

for both $\mathrm{m}=\mathrm{P}$ and $\mathrm{m}=\mathrm{L} 1$.

c. Treatment of the Liquid Spinodal Limit in the Temperature Inverse Calculation (Routine EOST). This method is based on the same concept as in EOSPEQ (Sec. 3.g). The liquid is not allowed to expand below the liquid spinodal density. Then if the pressure of the remaining components in the cell drops below the spinodal limit pressure PSpl,m, mechanical equilibrium cannot be achieved. In this case, the liquid is treated like a missing component that does not take part in the pressure equilibrium. 
In EOSPEQ, the component temperatures are fixed during the iteration so that the spinodal data (density and pressure) remain constant. Contrary to this, in EOST, both the temperature and the volume fraction (related to the microdensity by $\alpha=\bar{\rho} / \rho)$ are updated in each iteration step. Therefore, the spinodal data, which depend on temperature, must be updated by a call of the routine SPINDM at each iteration. Furthermore, to decide whether the updated state determined by $\Delta \mathrm{T}$ and $\Delta \alpha$ is a "spinodal limit" state or not, the relation [Eq. (3.30)] for each liquid component is used:

$$
\Delta T=\frac{\Delta e}{(\partial e / \partial T)_{\rho}}-\frac{(\partial e / \partial \ln p)_{T}}{(\partial e / \partial T)_{\rho}} \frac{\Delta \alpha}{\alpha}
$$

This means

$$
\Delta \mathrm{T}=\mathrm{c}_{1}+\mathrm{c}_{2} \Delta \alpha
$$

with known coefficients $c_{1}$ and $c_{2}$.

In the iteration step $k+1$, the Newton-Raphson procedure leads to the updated values

$$
\begin{aligned}
& T^{(k+1)}=T^{(k)}+\Delta T, \text { and } \\
& \alpha^{(k+1)}=\alpha^{(k)}+\Delta \alpha .
\end{aligned}
$$

The spinodal limit criterion puts a restriction on $\alpha^{k+1}$, the maximum allowable value being the value $\alpha_{S p l, m}(T)$ :

$$
\alpha^{k+1}=\min \left[\alpha^{k}+\Delta \alpha, \alpha_{S p l, m}\left(T^{k+1}\right)\right]
$$

At this stage, $\alpha_{\mathrm{Spl}, \mathrm{m}}(\mathrm{Tk}+1)$ can be determined only in first approximation by

$$
\alpha_{\mathrm{Spl}, \mathrm{m}}\left(\mathrm{T}^{\mathrm{k}+1}\right)=\alpha_{\mathrm{Spl,m}}\left(\mathrm{T}^{\mathrm{k}}\right)+\frac{\partial \alpha_{\mathrm{Spl}, \mathrm{m}}}{\partial \mathrm{T}} \Delta \mathrm{T}
$$


For consistency, the relation [Eq. (3.37)] must hold also in the case where $\Delta \alpha$ is determined by the spinodal limit. Therefore, one has to solve Eqs. (3.35) and (3.37) simultaneously using condition [Eq. (3.36)]. Inserting Eq. (3.35) into Eq. (3.37) gives

$$
\alpha_{\mathrm{Spl,m}}\left(\mathrm{T}^{k+1}\right)=\alpha_{\mathrm{Spl,m}}\left(\mathrm{T}^{\mathrm{k}}\right)+\mathrm{c}_{1} \frac{\partial \alpha_{\mathrm{Spl}, \mathrm{m}}}{\partial \mathrm{T}}+\mathrm{c}_{2} \frac{\partial \alpha_{\mathrm{Spl}, \mathrm{m}}}{\partial \mathrm{T}} \Delta \alpha
$$

Then the maximum $\Delta \alpha$ compatible with condition [Eq. (3.36)] is obtained by equating $\alpha_{\text {spl,m }}$ of Eq. (3.38) with $\alpha^{k}+\Delta \alpha$ :

$$
\alpha^{k}+\Delta \alpha_{\max }=\alpha_{S_{p l, m}}^{k}+c_{1} \frac{\partial \alpha_{\mathrm{Spl}, \mathrm{m}}}{\partial \mathrm{T}}+c_{2} \frac{\partial \alpha_{\mathrm{Spl,m}}}{\partial \mathrm{T}} \Delta \alpha_{\max }
$$

or

$$
\Delta \alpha_{\max }=\frac{\alpha_{\mathrm{Spl,m}}^{k}+c_{1} \frac{\partial \alpha_{\mathrm{Spl,m}}}{\partial \mathrm{T}}-\alpha^{k}}{1-c_{2} \frac{\partial \alpha_{\mathrm{Spl,m}}}{\partial \mathrm{T}}} .
$$

Therefore, $\Delta \alpha=\min \left(\Delta \alpha, \Delta \alpha_{\max }\right)$.

With this procedure, the spinodal limit criterion poses a limit on both $\Delta \alpha$ and $\Delta T$; the two quantities are related by Eq. (3.33). The derivative of $\alpha_{S p l, m}$ is

$$
\frac{\mathrm{d} \alpha_{\mathrm{Spl,m}}}{\mathrm{dT}}=-\frac{\alpha_{\mathrm{Spl,m}}}{\rho_{\mathrm{Spl,m}}} \frac{\mathrm{d} \rho_{\mathrm{Spl}, \mathrm{m}}}{\mathrm{dT}}
$$

The derivative of $\rho \mathrm{Spl}, \mathrm{m}$ can be obtained from the SPINDM routine.

The following steps are necessary in the routine EOST for treating liquid spinodal limits:

- At each step of the iteration, the routine SPINDM is called. The spinodal value $\rho$ Spl,m, $\mathrm{pSpl,m}$, and $\mathrm{d} \rho \mathrm{Spl}, \mathrm{m} / \mathrm{dT}$ are determined for the two liquids. From these data, $\alpha_{\mathrm{Spl}, \mathrm{m}}$ and $\mathrm{d} \alpha_{\mathrm{Spl}, \mathrm{m}} / \mathrm{dT}$ are calculated. 
- During the iteration, the maximum $\Delta \alpha$ allowed is $\Delta \alpha_{\max }$. If $\Delta \alpha=\Delta \alpha_{\max }$, the component present logical variable $\operatorname{LER}(m-1)$ is set to 0 , which means that the routine puts $\Delta \mathrm{p}_{\mathrm{m}}=0$ (missing component).

- As in EOSPEQ, $\Delta \mathrm{p}_{\mathrm{m}}{ }^{\text {eff }}$ is set to 0 if $\mathrm{pG}<\mathrm{pSpl,m}$ and $\alpha \mathrm{Geff} \leq \tilde{\alpha}$.

- If a cell is single phase, the component present variable is set to 1 at the beginning of the iteration, unless the component is truly missing, i.e., the macrodensity is low.

- The procedure during the single-phase iteration is similar to the one used with the two-phase iteration.

- As in EOSPEQ, the volume balance requires $\Sigma \Delta \alpha^{*} m=0$ in the singlephase iteration. Thus, if $\Delta \alpha_{m}$ is replaced by $\Delta \alpha_{\max }$, the difference must be added to either of the components $\mathrm{m}$ '.

6. EOS Function Partial Derivatives. The purpose of this chapter is to derive all the partial derivatives of the EOS functions denoted as $\bar{Y}$ with respect to the AFDM-independent variables $\bar{x}$, i.e., $\partial \bar{Y} / \partial \bar{x}$. Note that all the partial derivatives described here are not necessarily utilized in the AFDM thermohydraulic calculation.

a. EOS Function Dependencies. As a matter of convenience, an EOS function vector containing AFDM-dependent EOS variables that normally would be needed in a forward EOS evaluation is defined here.

$$
\begin{aligned}
\bar{Y}= & \left(p, e_{P}, e_{L 1}, e_{L 2}, e_{G 1}, e_{G 2}, e_{G 3}, e_{G}, T_{s 1}, T_{s 2}, e_{v a p, 1}, e_{v a p, 2}, h_{l g 1}, h_{l g}, \alpha_{P}, \alpha_{L 1},\right. \\
& \left.\alpha_{L 2}, \alpha_{G}\right),
\end{aligned}
$$

where the elements of $\bar{Y}$ vector are

p: cell pressure,

ep: internal energy of particle component,

$\mathrm{e}_{\mathrm{L} 1}$ : internal energy of liquid component one,

eL2: internal energy of liquid component two,

$e_{G 1}$ internal energy of vapor component one,

eG2: internal energy of vapor component two,

eG3: internal energy of noncondensable gas,

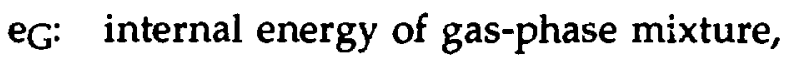

$T_{\mathrm{S} 1}$ : saturation temperature of liquid component one, 
$T_{s 2}$ : saturation temperature of liquid component two,

$e_{\text {vap, } 1:}$ saturation energy of vapor component one,

$e_{\text {vap,2: }}$ saturation energy of vapor component two,

$h_{\text {lg1 }}$ : heat of vaporization of liquid component one,

$\mathrm{h}_{\text {lg2: }}$ heat of vaporization of liquid component two,

$\alpha_{\mathrm{P}}$ : volume fraction of the particle component,

$\alpha_{L 1}:$ volume fraction of liquid component one,

$\alpha_{\mathrm{L} 2}$ : volume fraction of liquid component two, and

$\alpha_{G}$ : volume fraction of gas mixture.

As indicated in Sec. 1., the forward set of independent variables with which the AFDM solution algorithm proceeds is composed of macrodensities and temperatures (i.e., $\bar{\rho}$ and $T$ ). Thus, the independent variables vector can be written as

$$
\bar{X}=\left(\bar{\rho}_{P}, \bar{\rho}_{L 1}, \bar{\rho}_{L 2}, \bar{\rho}_{G 1}, \bar{\rho}_{G 2}, \bar{\rho}_{G 3}, T_{P}, T_{L 1}, T_{L 2}, T_{G}\right)
$$

Because the macrodensities are defined as $\bar{\rho}_{m}=\alpha_{m} \rho_{m}$ [Eq. (3.10)] for the liquid/ particle components $m=P, L .1, L 2$ and $\rho G M=\alpha_{G \text { eff }} \rho_{G M}$ [Eq. (3.11)] for the vapor components $M=1,2,3$, one needs to know the derivatives of the volume fraction $\partial \alpha / \partial \bar{\rho}$ and $\partial \alpha / \partial \mathrm{T}$ for every component. Saturation properties, such as the saturation energy or heat of vaporization, are functions of only the saturation temperatures, as described in Sec. 4. However, because the saturation temperature is a function of the vapor partial pressure and therefore a function of $\bar{\rho}$ and $T$, it is necessary to calculate the volume fraction derivatives $\partial \alpha / \partial \bar{\rho}$ and $\partial \alpha / \partial \mathrm{T}$ to get the derivatives of the saturated properties with respect to $\bar{\rho}$ and $T$.

b. Partial Derivatives of Volume Fractions. With the use of Eq. (3.10) for the liquid/particle component volume fractions, we obtain

$$
\frac{\partial \alpha_{m}}{\partial \alpha_{i}}=\frac{1}{\rho_{m}}\left(\frac{\partial \bar{\rho}_{m}}{\partial x_{i}}\right)-\frac{\bar{\rho}_{m}}{\rho_{m}^{2}}\left(\frac{\partial \rho_{m}}{\partial x_{i}}\right), m=P, L 1, L 2
$$

Note: This equation is valid only for the existing components. From the definition of the effective vapor volume fraction $\alpha_{\text {Geff: }}$ 


$$
\alpha_{\text {Geff }}=\max \left\{\alpha_{0}\left(1-\alpha_{s}\right), 1-\alpha_{s}-\left(1-\alpha_{0}\right)\left(\frac{\bar{\rho}_{\mathrm{P}}}{\rho_{\mathrm{P}}}+\frac{\bar{\rho}_{\mathrm{L} 1}}{\rho_{\mathrm{L} 1}}+\frac{\bar{\rho}_{\mathrm{L} 2}}{\rho_{\mathrm{L} 2}}\right)\right\},
$$

we obtain the derivatives of the effective vapor volume fraction as:

$$
\frac{\partial \alpha_{\text {Geff }}}{\partial x_{i}}=\left\{\begin{array}{l}
0 \text { for single-phase flow, } \\
-\left(1-\alpha_{0}\right) \sum_{m}\left\{\frac{1}{\rho_{m}}\left(\frac{\partial \bar{\rho}_{m}}{\partial x_{i}}\right)-\frac{\bar{\rho}_{m}}{\rho_{m}^{2}}\left(\frac{\partial \rho_{m}}{\partial x_{i}}\right)\right\}, \text { for two-phase flow }
\end{array}\right.
$$

where the summation is made over the existing component(s) $m$, and $x_{i}$ is the $i^{\text {th }}$ component of the independent variables vector of Eq. (3.39), and $i=1$ through 10:

$$
\frac{\partial \bar{\rho}_{m}}{\partial x_{i}}=\left\{\begin{array}{l}
0 \text { if } x_{i} \neq \bar{\rho}_{m} \\
1 \text { if } x_{j}=\bar{\rho}_{m}
\end{array} .\right.
$$

The $\left(\partial \rho_{m} / \partial x_{i}\right)$ 's are derived in Sec. 6.c. It is noted that in the case of the single-phase flow situation, Eq. (3.40) is evaluated based on the microdensity consistent with the cell pressure. Also, if $m$ indicates the missing component, then $\partial \alpha_{m} / \partial x_{i}=0$.

c. Partial Derivatives of Microdensities. The microdensity $\rho_{m}$ is derived from either two-phase or single-phase pressure equilibrium. For convenience, we formulate the partial derivatives of microdensities in two subsections, depending on which pressure equilibrium the microdensity satisfies.

(1) Two-phase equilibrium density derivatives. To obtain derivatives $\partial \rho_{m} / \partial x_{i}$, the two-phase pressure equilibrium statement [Eq. (3.9)] among all the existing components is used:

$$
\mathrm{P}_{\text {cell }}=\sum_{M=1}^{3} \mathrm{P}_{\mathrm{GM}}\left(\mathrm{P}_{\mathrm{GM}}, \mathrm{T}_{\mathrm{G}}\right)=\mathrm{p}_{\mathrm{m}}\left(\mathrm{P}_{\mathrm{m}}, \mathrm{T}_{\mathrm{m}}\right)
$$

where PGM is the partial pressure of the gas component $G M$, and $m$ indicates the existing liquid/particle component in the cell. From the above, we obtain

$$
\sum_{M=1}^{3}\left\{\left(\frac{\partial p_{G M}}{\partial \rho_{G M}}\right)_{T}\left(\frac{\partial \rho_{G M}}{\partial x_{i}}\right)+\left(\frac{\partial p_{G M}}{\partial T_{G}}\right)_{\rho}\left(\frac{\partial T_{G}}{\partial x_{1}}\right)\right\}=\left(\frac{\partial p_{m}}{\partial \rho_{m}}\right)_{T}\left(\frac{\partial p_{m}}{\partial x_{1}}\right)+\left(\frac{\partial p_{m}}{\partial T_{m}}\right)_{\rho}\left(\frac{\partial T_{m}}{\partial x_{1}}\right) \text {. }
$$


where

$$
\begin{aligned}
& i=1 \text { through } 10 \text {. Note that } \\
& \frac{\partial T_{G}}{\partial x_{i}}=\left\{\begin{array}{l}
1 \text { for } x_{i}=T_{G} \\
0 \text { for } x_{i} \neq T_{G}
\end{array}\right.
\end{aligned}
$$

and also that

$$
\frac{\partial T_{m}}{\partial x_{1}}=\left\{\begin{array}{l}
1 \text { for } x_{i}=T_{m} \\
0 \text { for } x_{i} \neq T_{m}
\end{array}\right.
$$

From Eq (3.42),

$$
\left(\frac{\partial p_{m}}{\partial x_{i}}\right)=\frac{\Pi-\left(\partial p_{m} / \partial T_{m}\right)\left(\partial T_{m} / \partial x_{1}\right)}{\left(\partial p_{m} / \partial p_{m}\right)}
$$

where

$$
\Pi=\sum_{\mathbf{m}}\left\{\left(\frac{\partial p_{\mathrm{GM}}}{\partial \rho_{\mathrm{GM}}}\right)_{\mathrm{T}}\left(\frac{\partial \rho_{\mathrm{GM}}}{\partial \mathrm{x}_{1}}\right)+\left(\frac{\partial \mathrm{p}_{\mathrm{GM}}}{\partial \mathrm{T}_{\mathrm{G}}}\right)_{\rho}\left(\frac{\partial \mathrm{T}_{\mathrm{G}}}{\partial \mathrm{x}_{1}}\right)\right\}
$$

From the definition of the microscopic density for the gas component,

$$
\rho_{\mathrm{GM}}=\bar{\rho}_{\mathrm{GM}} / \alpha_{\mathrm{ge}},
$$

where

$$
\begin{aligned}
\alpha_{\mathrm{ge}} & =1-\alpha_{\mathrm{s}}-\left(1-\alpha_{0}\right) \Sigma \alpha_{\mathrm{m}}, \text { and } \\
\mathrm{m} & =\mathrm{P}, \mathrm{L} 1, \mathrm{~L} 2
\end{aligned}
$$

we obtain in the case of two-phase pressure equilibrium

$$
\left(\frac{\partial \rho_{\mathrm{GM}}}{\partial \mathrm{x}_{\mathrm{i}}}\right)=\frac{1}{\alpha_{\mathrm{ge}}}\left(\frac{\partial \bar{\rho}_{\mathrm{GM}}}{\partial \mathrm{x}_{1}}\right)-\frac{\bar{\rho}_{\mathrm{GM}}}{\alpha_{\mathrm{ge}}^{2}}\left(\frac{\partial \alpha_{\mathrm{ge}}}{\partial \mathrm{x}_{\mathrm{i}}}\right) .
$$


Recalling that $\partial \alpha_{G}$ eff $/ \partial x_{i}$ [Eq. (3.41)] involves $\partial \rho_{m} / \partial x_{i}$ [Eq. (3.43)], we obtain $\partial \rho_{m} / \partial x_{i}$ by using Eqs. (3.41), (3.43), and (3.45). Note that $\alpha_{G \text { eff }}=\alpha_{\text {ge }}$ in the two-phase situation. For example, by substituting Eq. (3.41) into Eq. (3.45) and then substituting Eq. (3.45) into Eq. (3.44), we finally get a linear equation system for $\partial \rho_{m} / \partial x_{\mathbf{i}}$. The order of the linear equation system will be three, two, and one when there are three, two, and one liquid/particle components in the cell, respectively. After obtaining $\partial \rho_{m} / \partial x_{i}, \partial \alpha_{m} / \partial x_{i}, \partial \alpha_{m} / \partial x_{i}$, and $\partial \alpha_{G e f f} / \partial x_{i}$ can be calculated via Eqs. (3.40) and (3.41), respectively. Finally, $\partial \rho_{\mathrm{GM}} / \partial \mathrm{x}_{\mathbf{i}}$ is obtained from Eq. (3.45).

(2) Single-phase equilibrium density derivatives. In the same way as in the preceding section, the single-phase pressure equilibrium statement among all the existing components is used to de:ive $\partial \rho_{m} / \partial x_{i}$ :

$$
\begin{aligned}
\text { pcell } & =p\left(\rho_{p}, T_{p}\right), \\
& =p\left(\rho_{L 1}, T_{L 1}\right), \text { and } \\
& =p\left(\rho_{L 2}, T_{L 2}\right) .
\end{aligned}
$$

If one of the components $\mathrm{P}, \mathrm{L} 1$, or $\mathrm{L} 2$ is missing, then this component is excluded from Eq. (3.46). Here, we derive $\partial \rho_{m} / \partial x_{i}$ when all the liquid/particle components contribute to the mechanical equilibrium.

The pressure equilibrium [Eq. (3.46)] implies the following equations for the derivatives:

$$
\left(\frac{\partial p_{p}}{\partial \rho_{p}}\right)_{T}\left(\frac{\partial \rho_{p}}{\partial x_{1}}\right)+\left(\frac{\partial p_{P}}{\partial T_{P}}\right)_{p}\left(\frac{\partial T_{P}}{\partial x_{1}}\right)=\left(\frac{\partial p_{L 1}}{\partial \rho_{L 1}}\right)_{T}\left(\frac{\partial \rho_{L 1}}{\partial x_{1}}\right)+\left(\frac{\partial p_{L 1}}{\partial T_{L 1}}\right)_{p}\left(\frac{\partial T_{L 1}}{\partial x_{1}}\right)
$$

and

$$
\left(\frac{\partial \rho_{L 2}}{\partial \rho_{L 2}}\right)_{T}\left(\frac{\partial p_{L 2}}{\partial x_{1}}\right)+\left(\frac{\partial p_{L 2}}{\partial T_{L 2}}\right)_{\rho}\left(\frac{\partial T_{L 2}}{\partial x_{1}}\right)=\left(\frac{\partial p_{L 1}}{\partial \rho_{L 1}}\right)_{T}\left(\frac{\partial \rho_{L 1}}{\partial x_{1}}\right)+\left(\frac{\partial p_{L 1}}{\partial T_{L 1}}\right)_{\rho}\left(\frac{\partial T_{L 1}}{\partial x_{1}}\right) .
$$

For the single-phase flow, the following relationship is true:

$$
\frac{\partial \alpha_{1}}{\partial x_{1}}+\frac{\partial \alpha_{L_{1}}}{\partial x_{1}}+\frac{\partial \alpha_{L_{2}}}{\partial x_{1}}=0
$$


where $\alpha_{\mathrm{P}}, \alpha_{\mathrm{L} 1}$, and $\alpha_{\mathrm{L} 2}$ are starred volume fractions as defined in Sec. 3.a. However, the "*" is omitted for simplicity.

By substituting Eq. (3.40) into Eq. (3.49), we obtain

$$
\begin{aligned}
& \frac{\bar{\rho}_{P}}{\rho_{P}^{2}}\left(\frac{\partial \rho_{P}}{\partial x_{1}}\right)+\frac{\bar{\rho}_{L 1}}{\rho_{L 1}^{2}}\left(\frac{\partial \rho_{L 1}}{\partial x_{1}}\right)+\frac{\bar{\rho}_{L 2}}{\rho_{L 2}^{2}}\left(\frac{\partial \rho_{L 2}}{\partial x_{1}}\right)= \\
& \frac{1}{\rho_{P}}\left(\frac{\partial \bar{\rho}_{P}}{\partial x_{1}}\right)+\frac{1}{\rho_{L 1}}\left(\frac{\partial \bar{\rho}_{L 1}}{\partial x_{1}}\right)+\frac{1}{\rho_{L 2}}\left(\frac{\partial \bar{\rho}_{L 2}}{\partial x_{1}}\right) .
\end{aligned}
$$

Equations (3.47), (3.48), and (3.50) form a linear algebraic equation for $\partial \rho_{p} / \partial x_{i}$, $\partial \rho_{\mathrm{L} 1} / \partial \mathrm{x}_{\mathbf{i}}$, and $\partial \rho_{\mathrm{L} 2} / \partial \mathrm{x}_{\mathbf{i}}$ and can be solved by the standard procedure.

If one of the three components is missing, the final equations to be solved will be a $2 \times 2$ matrix equation, whereas Eqs. (3.47), (3.48), and (3.50) are represented by a $3 \times 3$ matrix equation. If only one component stays in the cell, this case is trivial because the volume fraction is constant. In this case,

$$
\frac{\partial \rho_{m}}{\partial x_{1}}=\frac{1}{\alpha_{m}}\left(\frac{\partial \bar{\rho}_{m}}{\partial x_{1}}\right)
$$

d. Partial Derivatives of Cell Pressure. For the same reason as in Sec. 6.c, we derive the partial derivatives of the cell pressure in the following two subsections.

(1) Two-phase equilibrium pressure derivatives. Because of the pressure dependency on the microdensity and the temperature, the pressure derivatives are given by

$$
\frac{\partial \mathrm{p}_{\mathrm{cell}}}{\partial x_{1}}=\sum_{M=1}^{3} \frac{\partial \mathrm{p}_{\mathrm{GM}}}{\partial \mathrm{x}_{1}}=\sum_{M}\left[\left(\frac{\partial \mathrm{p}_{\mathrm{GM}}}{\partial \rho_{\mathrm{GM}}}\right)_{\mathrm{T}}\left(\frac{\partial \mathrm{p}_{\mathrm{GM}}}{\partial \mathrm{x}_{\mathrm{i}}}\right)+\left(\frac{\partial \mathrm{p}_{\mathrm{GM}}}{\partial \mathrm{T}_{\mathrm{G}}}\right)_{\rho}\left(\frac{\partial \mathrm{T}_{\mathrm{G}}}{\partial \mathrm{x}_{1}}\right)\right] .
$$

The term $\partial \rho_{\mathrm{GM}} / \partial x_{i}$ is already obtained by Eq. (3.45). Note that if $x_{i}$ is associated with the missing component, the $\partial \mathrm{p}_{\text {cell }} / \partial \mathrm{x}_{\mathrm{i}}=0$.

(2) Single-phase equilibrium pressure derivatives. For example, when all the liquid/particle components contribute to the single-phase pressure equilibrium, 


$$
\frac{\partial p_{\text {cell }}}{\partial x_{1}}=\frac{\partial p_{L 2}}{\partial x_{1}}=\left(\frac{\partial p_{L 2}}{\partial p_{L 2}}\right)_{T}\left(\frac{\partial p_{L 2}}{\partial x_{1}}\right)+\left(\frac{\partial p_{L 2}}{\partial T_{L 2}}\right)_{\rho}\left(\frac{\partial T_{L 2}}{\partial x_{1}}\right) .
$$

Here, the thermodynamic pressure of the $\mathbf{L} 2$ component is taken as the representative. $\partial \rho_{\mathrm{L} 2} / \partial x_{\mathbf{i}}$ is already given in Sec. 6.c. If the other components are taken as a reference pressure, the same pressure derivatives are to be obtained. If $\mathbf{x}_{\mathbf{i}}$ is associated with the missing components, the $\partial \mathrm{p}_{\text {cell }} / \partial \mathrm{x}_{\mathrm{i}}=0$. Also, note that $\partial \mathrm{p}_{\text {cell }} / \partial \bar{\rho} \mathrm{GM}$ $\partial \mathrm{p}_{\mathrm{cell}} / \partial \mathrm{T}_{\mathrm{G}}=0$.

\section{e. Partial Derivatives of Internal Energies}

(1) Particle and liquid internal energy derivatives. For the internal energies obtained as the result of the two-phase pressure equilibrium, the derivatives may be written as

$$
\frac{\partial e_{m}}{\partial x_{1}}=\left(\frac{\partial e_{m}}{\partial \rho_{m}}\right)_{T}\left(\frac{\partial \rho_{m}}{\partial x_{1}}\right)+\left(\frac{\partial e_{m}}{\partial T_{m}}\right)\left(\frac{\partial T_{m}}{\partial x_{1}}\right), m=P, L 1, L 2
$$

The term $\partial \rho_{m} / \partial x_{i}$ is defined in Sec. 6.c.

For the internal energies obtained as the result of the single-phase pressure equilibrium, Eq. (3.51) can be used as the derivative expression but with $\partial \rho_{m} / \partial x_{i}$ defined in Sec. 6.c.

Special care is taken when $\mathrm{m}$ in Eq. (3.51) represents a missing component. As was explained in Sec. 3.b, the internal energy of the missing component is assumed to be a function of the given temperature. Therefore,

$$
\frac{\partial e_{m}}{\partial x_{1}}=\left\{\begin{array}{l}
0 \text { for } x_{i} \neq T_{m} \\
\left(\frac{\partial e_{m}}{\partial T_{m}}\right)_{S a t} \text { for } x_{i}=T_{m}
\end{array}\right.
$$

where the subscript Sat implies that the derivative is taken along the saturation dome of the material component $\mathrm{m}$.

(2) Mixture gas internal energy derivatives. Taking the derivative of Eq. (3.5) with respect to $x_{i}$ yields 


$$
\begin{aligned}
& \frac{\partial e_{G}}{\partial x_{1}}=\sum_{M=1}^{3}\left[\frac{\partial w_{M}}{\partial x_{1}} e_{G M}+w_{M} \frac{\partial e_{G M}}{\partial x_{1}}\right] \text {, where } \\
& \frac{\partial w_{M}}{\partial x_{1}}=\frac{\partial \bar{\rho}_{G M} / \partial x_{1}}{\sum_{M=1}^{3} \bar{\rho}_{g k}}-\frac{\bar{\rho}_{G M}}{\left(\sum_{M=1}^{3} \bar{\rho}_{g k}\right)^{2}} \sum_{k=1}^{3}\left(\frac{\partial \bar{\rho}_{g k}}{\partial x_{1}}\right) \text {, and } \\
& \frac{\partial e_{G M}}{\partial x_{1}}=\left(\frac{\partial e_{G M}}{\partial \rho_{G M}}\right)_{T}\left(\frac{\partial \rho_{G M}}{\partial x_{1}}\right)+\left(\frac{\partial e_{G M}}{\partial T_{G}}\right)_{\rho}\left(\frac{\partial T_{G}}{\partial x_{i}}\right) .
\end{aligned}
$$

The term $\partial \rho_{\mathrm{GM}} / \partial \mathrm{x}_{\mathrm{i}}$ is defined by Eq. (3.52) in Sec. 6.c, and $\partial \mathrm{T}_{\mathrm{G}} / \partial \mathrm{x}_{\mathrm{i}}$ is nonzero only for $\mathrm{i}=\mathbf{1 0}\left(\mathrm{x}_{10}=\mathrm{T}_{\mathrm{G}}\right)$.

(3) Individual gas internal energy derivatives. Equation (3.53) gives the partial derivatives of the individual gas internal energy with respect to $x_{i}$.

\section{f. Partial Derivatives of Saturation Properties}

(1) Saturation temperature derivatives. Because the saturation temperature of a liquid component is assumed to depend only on the partial pressure of the corresponding vapor component (see Sec. 4.a), the partial derivatives are given by

$$
\frac{\partial \mathrm{T}_{\mathrm{Sat}, \mathrm{M}}}{\partial \mathrm{x}_{\mathrm{i}}}=\frac{\partial \mathrm{T}_{\mathrm{Sat}, \mathrm{M}}}{\partial \mathrm{p}_{\mathrm{GM}}}\left[\left(\frac{\partial \mathrm{p}_{\mathrm{GM}}}{\partial \rho_{G M}}\right)_{\mathrm{T}}\left(\frac{\partial \rho_{\mathrm{GM}}}{\partial \mathrm{x}_{1}}\right)+\left(\frac{\partial \mathrm{p}_{\mathrm{GM}}}{\partial \mathrm{T}_{\mathrm{G}}}\right)_{\rho}\left(\frac{\partial \mathrm{T}_{\mathrm{G}}}{\partial \mathrm{x}_{\mathrm{i}}}\right)\right],
$$

where

$$
M=1 \text { and } 2 .
$$

$\mathrm{T}_{\mathrm{Sat}} \mathrm{M}$ is the saturation temperature of liquid component $\mathrm{M}$. The term $\partial \rho_{G M} / \partial \mathrm{x}_{i}$ is defined in Sec. 6.c. The term $\partial \mathrm{T}_{\mathrm{Sat}, \mathrm{M}} / \partial \mathrm{p}_{\mathrm{GM}}$ is obtained directly from the SESAME401 table.

(2) Other saturation property derivatives. Provided that the derivatives of the saturation temperature are known, it is straightforward to obtain the following: 
- liquid saturation energy derivatives

$$
\frac{\partial e_{\text {Con,M }}}{\partial x_{1}}=\frac{\partial e_{\text {Con,M }}}{\partial T_{\text {Sat,M }}} \frac{\partial \mathrm{T}_{\text {Sat,M }}}{\partial x_{1}}
$$

where

$$
\text { e Con, } M=\text { the saturation energy of liquid component } M \text {. }
$$

$\frac{\partial \mathrm{T}_{\mathrm{Sat}, \mathrm{M}}}{\partial \mathrm{x}_{1}}$ is given by Eq. (3.54).

- vapor saturation energy derivatives

$$
\frac{\partial \mathrm{e}_{\mathrm{V}_{\mathrm{ap}, M}}}{\partial \mathrm{x}_{1}}=\frac{\partial \mathrm{e}_{\mathrm{V}, \mathrm{M}, \mathrm{M}}}{\partial \mathrm{T}_{\mathrm{Sat}, M}} \frac{\partial \mathrm{T}_{\mathrm{Sat}, \mathrm{M}}}{\partial \mathrm{x}_{1}}
$$

where

eVap,M = the saturation energy of the vapor component GM.

- heat of vaporization derivatives are:

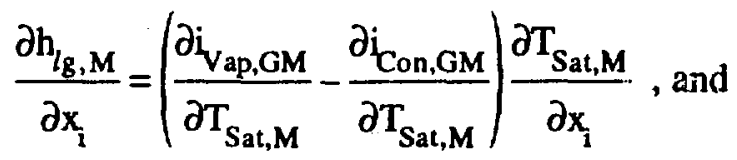

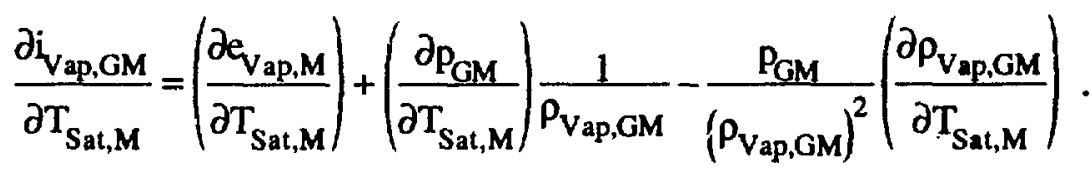

iVap,GM, PGM, and $\rho$ Vap, GM are the saturation enthalpy, the partial pressure, and the saturation density of the vapor component GM:

$$
\frac{\partial \mathrm{i}_{\text {Con }, M}}{\partial \mathrm{T}_{\text {Sat, }, M}}=\left(\frac{\partial \mathrm{e}_{\text {Con }, M}}{\partial \mathrm{T}_{\text {Sat, }, M}}\right)+\left(\frac{\partial \mathrm{p}_{\mathrm{GM}}}{\partial \mathrm{T}_{\text {Sat, }}}\right) \frac{1}{\rho_{\mathrm{Con}, M}}-\frac{\mathrm{p}_{\mathrm{GM}}}{\left(\rho_{\mathrm{Con}, M}\right)^{2}}\left(\frac{\partial \rho_{\text {Con }, M}}{\partial \mathrm{T}_{\text {Sat }, M}}\right) .
$$


The terms iCon, $M$ and $P C o n, M$ are the saturation enthalpy and the saturation density of liquid component $M$.

g. Change of Independent Variables. The AFDM algorithm utilizes a change of independent variables for the density derivatives used in solving the gas, energy, and momentum equations and the liquid-one momentum equation. This change of variables is physically a constraint in the composition of a mixture that requires the mass fractions $x_{m}$ associated with a mixture to be constant, i.e.,

$$
x_{m}=\bar{\rho}_{m} / \sum_{m} \bar{\rho}_{m}=\text { constant }
$$

There are two mixtures impacted by this constraint. One is the vapor mixture as explained in Sec. 2.b, and the other is the liquid/particle $(\mathrm{L} 1+\mathrm{P})$ mixture.

The gas internal energy density derivative is first addressed with the result

$$
\frac{\partial e_{G}}{\partial \bar{\rho}_{G}}=\sum_{M=1}^{3}\left(\frac{\partial e_{G}}{\partial \bar{\rho}_{G M}}\right) X_{M},
$$

which follows directly from the chain rule and Eq. (3.55). Here,

$$
\bar{\rho}_{\mathrm{G}}=\sum_{M=1}^{3} \bar{\rho}_{\mathrm{GM}} .
$$

The cell pressure density derivative also follows as

$$
\frac{\partial p_{c e l l}}{\partial \bar{\rho}_{G}}=\sum_{M=1}^{3}\left(\frac{\partial p_{c e l l}}{\partial \bar{\rho}_{G M}}\right) x_{M}
$$

The terms $\left(\partial \mathrm{e}_{\mathrm{G}} / \partial \bar{\rho}_{\mathrm{GM}}\right)$ and $\left(\partial \mathrm{p}_{\mathrm{cell}} / \partial \bar{\rho}_{\mathrm{GM}}\right)$ already have been developed in Secs. 6.c and 6.d.

The liquid-one-momentum equation and particle mixture pressure derivative have a form similar to Eq. (3.56).

$$
\frac{\partial p_{c e l l}}{\partial \bar{\rho}_{\mathrm{P}+L 1}}=\frac{\partial p_{c e l 1}}{\partial \bar{\rho}_{L 1}} x_{L 1}+\frac{\partial p_{c e l 1}}{\partial \bar{\rho}_{p}} x_{p}
$$


where

$$
\bar{\rho}_{\mathrm{P}+\mathrm{L} 1}=\bar{\rho}_{\mathrm{P}}+\bar{\rho}_{\mathrm{L} 1} \text {. }
$$

Because the gas energy component is also a function of the liquid-one ard particle densities (via $\alpha_{G}$ ), $e_{G}$ also is affected by this composition constraint $X_{L 1}$ and $X_{P}$ :

$$
\frac{\partial e_{G}}{\partial \bar{\rho}_{P+L 1}}=\frac{\partial e_{G}}{\partial \bar{\rho}_{L 1}} x_{L 1}+\frac{\partial e_{G}}{\partial \bar{\rho}_{P}} x_{P}
$$

\section{The TEOS Property Derivatives}

1. Introduction. In steps 1 and 4 of the AFDM algorithm, the NewtonRaphson method is used to solve the equations for the heat- and mass-transfer models, as well as for the pressure iteration. This is why numerous partial derivatives are needed, especially for the physical properties that come from EOS. To invert EOS, even more derivatives are needed, as is explained in the preceding Sec. B.

2. Derivatives Dependencies. All physical properties needed in AFDM calculations (mainly internal energies and pressures) are functions of the temperatures and microdensities for each component, namely particles, liquid 1, liquid 2, vapor 1, vapor 2, and an incondensable gas.

These quantities are derived by interpolating the 301 SESAME data tables, where the internal energy and the pressure of every component are given as a function of temperature $T$ and microdensity $\rho$. The results of this interpolation process are given in Table $\mathrm{I}$.

The independent variables used in AFDM are the temperatures $\mathrm{T}$ and macrodensities $\bar{\rho}$ where $\rho=\bar{\rho} / \alpha$ (macrodensity divided by the volume fraction). Every time a derivative is calculated with respect to AFDM-independent variables $(\rho, T)$, the derivatives of the volume fractions $\partial \alpha / \partial \bar{\rho}$ and $\partial \alpha / \partial \mathrm{T}$ for every component must be known.

For the saturation properties, such as the saturated energies or the saturation pressures, the calculation path is different because these properties depend only on the saturation temperature.

For instance, if the saturation pressure pSat or the saturated vapor internal energy eVap is needed, one has to interpolate the 401 SESAME data tables where 


\section{TABLE I \\ OUTPUT OF INTERPOLATION (301 SESAME TABLE)}

Input: $\quad \rho$ and $T$

Output: $\mathrm{p}$ pressure

$\partial \mathrm{p} / \partial \rho$ pressure derivative with respect to microdensity at a constant temperature

$\partial \mathrm{p} / \partial \mathrm{T}$ pressure derivative with respect to temperature at a constant microdensity

e specific internal energy

$\partial e / \partial \rho$ specific internal energy derivative with respect to microdensity at a constant temperature

$\partial \mathrm{e} / \partial \mathrm{T}$ specific internal energy derivative with respect to temperature at a constant microdensity

Remark: Interpolation routines yield derivatives with respect to the $\log$ of $\rho$ and $T$.

PSat and evap are given as functions of $\mathrm{T}_{\text {Sat. }}$ This interpolation is done by the VAPDOM routine. The results of this interpolation process are given in Table II.

3. Volume Fraction Derivatives. In every numerical cell, an effective gas volume fraction, $\alpha_{\mathrm{Geff}}$, is defined as

$$
\alpha_{\mathrm{Geff}}=1-\alpha_{s}-\left(1-\alpha_{0}\right)\left(\alpha_{P}+\alpha_{\mathrm{L} 1}+\alpha_{\mathrm{L} 2}\right)
$$

where

$$
\begin{aligned}
& \bar{\rho}_{s} / \rho_{s}=\alpha_{s} \quad \text { structure volume fraction, } \\
& \bar{\rho}_{\mathrm{P}} / \rho_{\mathrm{P}}=\alpha_{\mathrm{P}} \quad \text { particle volume fraction, } \\
& \bar{\rho}_{\mathrm{L} 1} / \rho_{\mathrm{L} 1}=\alpha_{\mathrm{L} 1} \text { liquid } 1 \text { volume fraction, } \\
& \bar{\rho}_{L 2} / \rho_{L 2}=\alpha_{L_{2}} \text { liquid } 2 \text { volume fraction, and } \\
& \alpha_{0} \\
& \text { user-defined residual gas volume fraction. }
\end{aligned}
$$

All these derivatives are calculated along the saturation curve. 
TABLE II

OUTPUT OF INTERPOLATION (401 SESAME DATA TABLE)

Pressure given

$\mathrm{T}_{\text {Sat }}$

$(\partial T / \partial \mathrm{p})_{\text {Sat }}$ or
Temperature given

PSat

$(\partial \mathrm{p} / \partial \mathrm{T})_{\mathrm{Sat}}$

$$
\begin{aligned}
& \rho_{\text {Vap }} \\
& \left(\partial \rho_{\text {Vap }} / \partial T\right)_{S a t}
\end{aligned}
$$

$\rho_{\text {Con }}$

$\left(\partial \rho_{\text {Con }} / \partial \mathrm{T}\right)_{\mathrm{Sat}}$

eVap

$(\partial e v a p / \partial T)_{S a t}$

econ

$\left(\partial \mathrm{Con}_{\mathrm{C}} / \partial \mathrm{T}\right)_{\mathrm{Sat}}$

ivap

$\left(\frac{\partial i_{\text {ap }}}{\partial T}\right)_{\text {Sat }}$

icon

$\left(\frac{\partial \mathrm{i}_{\mathrm{Con}}}{\partial \mathrm{T}}\right)_{\text {Sat }}$
Saturated vapor density

Saturated vapor density derivative with respect to temperature

Saturated iiquid density

Saturated liquid density derivative with respect to temperature

Saturated vapor internal energy

Saturated vapor internal energy derivative with respect to temperature

Saturated liquid internal energy

Saturated liquid internal energy derivative with respect to temperature

Saturated vapor enthalpy

Saturated vapor enthalpy derivative with respect to temperature

Saturated liquid enthalpy

Saturated liquid enthalpy derivative with respect to temperature 
The numerical cell is supposed to be in single-phase or two-phase configuration, depending upon a condition on $\alpha_{G \text { Geff: }}$

$$
\left(1-\alpha_{s}\right) \alpha_{0} \geq \alpha_{G e f f} \text { or }\left(1-\alpha_{s}\right) \alpha_{0}<\alpha_{G e f f}
$$

Because every component in each cell is presumed to be in pressure equilibrium with the other components, obviously the volume fractions depend on the pressure and the associated derivatives.

\section{a. Two-Phase Calculation.}

\section{Gas Components}

As an example, in this section, the calculation of $\partial \alpha_{G e f f} / \partial \bar{\rho}_{m}(m=P, L 1, L 2)$ is given. From the definition of $\alpha_{\text {Geff, }}$ we obtain

$$
\frac{\partial \alpha_{\text {Geff }}}{\partial \bar{\rho}_{m}}=-\left(1-\alpha_{0}\right)\left[\frac{1}{\rho_{m}}-\sum_{m^{\prime}} \frac{\bar{\rho}_{m^{\prime}}}{\rho_{m^{\prime}}^{2}} \frac{\partial \rho_{m^{\prime}}}{\partial \bar{\rho}_{m}}\right], m^{\prime}=P, L 1, L 2 .
$$

To get the derivatives $\partial \rho_{m^{\prime}} / \partial \bar{\rho}_{m}$, the pressure equilibrium statement between all the components is used in each cell:

$$
p_{g}=\sum_{n} p_{n}\left(\rho_{n}, T_{g}\right)=p_{m},\left(\rho_{m}, T_{m}\right)
$$

where

$$
\begin{aligned}
\mathrm{n} & =\mathrm{g} 1, \mathrm{~g} 2, \mathrm{~g} 3, \text { and } \\
\mathrm{m}^{\prime} & =\mathrm{P}, \mathrm{L} 1, \mathrm{~L} 2 .
\end{aligned}
$$

The derivatives of all variables in Eq. (3.58) are related by

$$
\sum_{n}\left[\frac{\partial p_{n} \partial \rho_{n}}{\partial \rho_{n} \partial \bar{\rho}_{m}}+\frac{\partial p_{n} \partial T_{g}}{\partial T_{g} \partial \bar{\rho}_{m}}\right]=\frac{\partial p_{m^{\prime}}}{\partial \rho_{m^{\prime}}} \frac{\partial \rho_{m^{\prime}}}{\partial \bar{\rho}_{m}}+\frac{\partial p_{m^{\prime}}}{\partial T_{m^{\prime}}} \frac{\partial T_{m^{\prime}}}{\partial \bar{\rho}_{m}}
$$


Because $\bar{\rho}_{\mathrm{i}^{\prime}} \bar{\rho}_{\mathrm{m}}, \mathrm{T}_{\mathrm{g}}$, and $\mathrm{T}_{\mathrm{m}}$ or $\mathrm{T}_{\mathrm{m}^{\prime}}$ are independent variables, the derivatives $\partial \mathrm{T}_{\mathrm{g}} / \partial \bar{\rho}_{\mathrm{m}}$ and $\partial \mathrm{T}_{\mathrm{m}^{\prime}} / \partial \bar{\rho}_{\mathrm{m}}$ are zero in Eq. (3.59). Consequently,

$$
\frac{\partial \rho_{m^{\prime}}}{\partial \bar{\rho}_{m}}=\left[\sum_{n} \frac{\partial p_{n}}{\partial \rho_{n}} \frac{\partial \rho_{n}}{\partial \bar{\rho}_{m}}\right] / \frac{\partial p_{m^{\prime}}}{\partial \rho_{m^{\prime}}}
$$

From the definition of the macroscopic density for the gas component,

$$
\begin{gathered}
\rho_{\mathrm{n}}=\bar{\rho}_{\mathrm{n}} / \alpha_{\mathrm{Geff}}, \text { and } \\
\frac{\partial \rho_{\mathrm{n}}}{\partial \bar{\rho}_{\mathrm{m}}}=-\frac{\bar{\rho}_{\mathrm{n}}}{\alpha_{\mathrm{Geff}}^{2}} \frac{\partial \alpha_{\text {Geff }}}{\partial \bar{\rho}_{\mathrm{m}}} .
\end{gathered}
$$

By using Eqs. (3.57), (3.60), and (3.61), the expression of the derivatives $\partial \alpha_{G e f f} / \partial \bar{\rho}_{m}$ is obtained:

$$
\frac{\partial \alpha_{\text {Geff }}}{\partial \bar{\rho}_{m}}=\frac{\alpha_{0}-1}{\rho_{m}} /\left\{1+\frac{\left(1-\alpha_{0}\right)}{\alpha_{\text {Geff }}^{2}}\left[\sum_{n} \frac{\partial P_{n}}{\partial \rho_{n}} \bar{\rho}_{n}\right]\left[\sum_{m^{\prime}} \frac{\bar{\rho}_{m^{\prime}}}{\rho_{m^{\prime}}} \frac{\partial}{\partial P_{m^{\prime}}} \frac{1}{\partial \rho_{m^{\prime}}}\right]\right\}
$$

where

$$
\begin{aligned}
\mathrm{n} & =\mathrm{g} 1, \mathrm{~g} 2, \mathrm{~g} 3 \text { and } \\
\mathrm{m}^{\prime} & =\mathrm{m}=\mathrm{P}, \mathrm{L} 1, \mathrm{~L} 2 .
\end{aligned}
$$

To face all the possible situations, the configuration where one or two "liquid" components are missing has to be taken into account. In Eq. (3.57), m' represents only the components that are present in the numerical cell. The threeequation system [Eq. (3.58)] is reduced to one or two equations, depending on the number of liquid components. As a consequence, in Eq. (3.62), the missing components are not involved. All volume fraction derivatives with respect to temperature or macrodensity of a missing component are set equal to zero. The subscripts $n, m, m^{\prime}$, and $k$ are defined in Table III.1; the list of $\alpha_{\text {Geff }}$ derivatives is given in Table III.2. 


\section{TABLE III.1}

TWO-PHASE SITUATIONS

\begin{tabular}{|l|}
\hline 4 components \\
\hline $\mathrm{g}, \mathrm{P}, \mathrm{L} 1, \mathrm{~L} 2$ \\
$\mathrm{n}^{\prime}=\mathrm{n}=\mathrm{g} 1, \mathrm{~g} 2, \mathrm{~g} 3$ \\
$\mathrm{~m}=\mathrm{P}, \mathrm{L} 1, \mathrm{~L} 2$ \\
$\mathrm{~m}^{\prime}=\mathrm{P}, \mathrm{L} 1, \mathrm{~L} 2$ \\
\hline
\end{tabular}

\begin{tabular}{|l|}
\hline 3 components \\
\hline $\mathrm{g}, \mathrm{P}, \mathrm{L} 1$ \\
$\mathrm{n}^{\prime}=\mathrm{n}=\mathrm{g} 1, \mathrm{~g} 2, \mathrm{~g} 3$ \\
$\mathrm{~m}=\mathrm{m}^{\prime}=\mathrm{P}, \mathrm{L} 1$ \\
missing components: \\
$\mathrm{k}=\mathrm{L} 2$
\end{tabular}

\begin{tabular}{|l|}
\hline 2 components \\
\hline$g, P$ \\
$n^{\prime}=n=g 1, g 2, g 3$ \\
$m^{\prime}=m=P$ \\
missing components: \\
$k=L 1, L 2$ \\
\hline
\end{tabular}

\begin{tabular}{|l|}
\hline 3 components \\
\hline $\mathrm{g}, \mathrm{P}, \mathrm{L} 2$ \\
$\mathrm{n}^{\prime}=\mathrm{n}=\mathrm{g} 1, \mathrm{~g} 2, \mathrm{~g} 3$ \\
$\mathrm{~m}=\mathrm{m}^{\prime}=\mathrm{P}, \mathrm{L} 2$ \\
missing components: \\
$\mathrm{k}=\mathrm{L} 1$
\end{tabular}

\section{2 components}

g, L1

$n^{\prime}=\mathrm{n}=\mathrm{g} 1, \mathrm{~g} 2, \mathrm{~g} 3$

$\mathrm{m}^{\prime}=\mathrm{m}=\mathrm{L} 1$

missing components:

$\mathrm{k}=\mathrm{P}, \mathrm{L} 2$

\begin{tabular}{|l|}
\hline 3 components \\
\hline $\mathrm{g}, \mathrm{L} 1, \mathrm{~L} 2$ \\
$\mathrm{n}^{\prime}=\mathrm{n}=\mathrm{g} 1, \mathrm{~g} 2, \mathrm{~g} 3$ \\
$\mathrm{~m}=\mathrm{m}^{\prime}=\mathrm{L} 1, \mathrm{~L} 2$ \\
missing components: \\
$\mathrm{k}=\mathrm{P}$
\end{tabular}

\begin{tabular}{|l|}
\hline 2 components \\
\hline $\mathrm{g}, \mathrm{L} 2$ \\
$\mathrm{n}^{\prime}=\mathrm{n}=\mathrm{g} 1, \mathrm{~g} 2, \mathrm{~g} 3$ \\
$\mathrm{~m}^{\prime}=\mathrm{m}=\mathrm{L} 2$ \\
missing components: \\
$\mathrm{k}=\mathrm{P}, \mathrm{L} 1$
\end{tabular}

\section{Particle and Liquid Component Volume Fractions}

By using the pressure equilibrium statements [Eq. (3.58)], the derivatives of particle and liquid component volume fractions $\partial \alpha_{m^{\prime}} / \partial \bar{\rho}_{m}, \partial \alpha_{m^{\prime}} / \partial \bar{\rho}_{n}, \partial \alpha_{m^{\prime}} / \partial T_{m}$ and $\partial \alpha_{m^{\prime}} / \partial T_{g}\left(m=m^{\prime}=P, L 1, L 2\right)$ can be expressed easily as functions of the $\alpha_{G \text { Geff }}$ derivatives. As an example, the calculation for $\partial \alpha_{\mathrm{m}} / \partial \bar{\rho}_{\mathrm{m}}$ is given. As quoted in Eq. (3.59),

$$
\sum_{n} \frac{\partial p_{n}}{\partial \rho_{n}} \frac{\partial \rho_{n}}{\partial \bar{\rho}_{m}}=\frac{\partial p_{m^{\prime}}}{\partial \rho_{m^{\prime}}} \frac{\partial \rho_{m^{\prime}}}{\partial \bar{\rho}_{m}}
$$


TABLE III.2

TWO-PHASE SITUATION: EFFECTIVE GAS VOLUME FRACTION DERIVATIVES

$$
\begin{aligned}
& \frac{\partial \alpha_{\text {Geff }}}{\partial \bar{\rho}_{m}}=\frac{\frac{\alpha_{0}-1}{\rho_{m}}}{D} \\
& \frac{\partial \alpha_{\text {Geff }}}{\partial \bar{\rho}_{n}}=\frac{\frac{\left(1-\alpha_{0}\right)}{\alpha_{G_{\text {Geff }}}} \frac{\partial p_{n^{n}}}{\partial \rho_{n}}\left[\sum_{m^{\prime}} \frac{\bar{\rho}_{\mathrm{m}^{\prime}}}{\rho_{m^{\prime}}^{2} \frac{1}{\partial p_{m^{\prime}}}} \frac{\partial \rho_{m^{\prime}}}{D}\right]}{D} \\
& \left(\alpha_{0}-1\right) \frac{\bar{\rho}_{m}}{\rho_{m}^{2}} \frac{1}{\partial p_{m}} \frac{\partial p_{m}}{\partial T_{m}} \\
& \frac{\partial \alpha_{\text {Geff }}}{\partial T_{m}}=\frac{\partial \rho_{m}}{D} \\
& \frac{\partial \alpha_{G \text { eff }}}{\partial T_{g}}=\frac{\left(1-\alpha_{0}\right)\left[\sum_{n^{\prime}} \frac{\partial p_{n^{\prime}}}{\partial T_{g}}\right]\left[\sum_{m^{\prime}} \frac{\bar{p}_{m^{\prime}}}{p_{m^{\prime}} \frac{1}{\partial p_{m^{\prime}}}} \frac{\partial p_{m^{\prime}}}{d}\right]}{D} \\
& \frac{\partial \alpha_{\text {Geff }}}{\partial \bar{\rho}_{k}}=0 \\
& \frac{\partial \alpha_{\text {Geff }}}{\partial \mathrm{T}_{\mathrm{k}}}=0 \\
& D=\left\{1+\frac{\left(1-\alpha_{0}\right)}{\alpha_{\text {Geff }}^{2}}\left[\sum_{n^{\prime}} \frac{\partial p_{n^{\prime}}}{\partial \rho_{n^{\prime}}} \bar{\rho}_{n^{\prime}}\right]\left[\sum_{m^{\prime}} \frac{\bar{\rho}_{m^{\prime}}}{\rho_{m^{\prime}}^{2}} \frac{1}{\partial p_{m^{\prime}}} \frac{\partial \rho_{m^{\prime}}}{\partial x^{\prime}}\right]\right\}
\end{aligned}
$$


The microscopic densities for the liquid and vapor components are given by

$$
\begin{aligned}
& \rho_{\mathrm{m}^{\prime}}=\frac{\bar{\rho}_{\mathrm{m}^{\prime}}}{\alpha_{\mathrm{m}^{\prime}}} \text {, and } \\
& \rho_{\mathrm{n}}=\frac{\bar{\rho}_{\mathrm{n}}}{\alpha_{\mathrm{Geff}}} .
\end{aligned}
$$

These densities can be differentiated, yielding

$$
\begin{aligned}
& \frac{\partial \rho_{m^{\prime}}}{\partial \bar{\rho}_{m}}=\frac{1}{\alpha_{m^{\prime}}} \delta\left(m, m^{\prime}\right)-\frac{\bar{\rho}_{m^{\prime}} \partial \alpha_{m^{\prime}}}{\alpha_{m^{2}}^{2} \partial \bar{\rho}_{m}} \text {, and } \\
& \frac{\partial \rho_{n}}{\partial \bar{\rho}_{m}}=-\frac{\bar{\rho}_{n}}{\alpha_{\text {Geff }}^{2}} \frac{\partial \alpha_{G_{\text {Geff }}}}{\partial \bar{\rho}_{m}}
\end{aligned}
$$

where

$$
\begin{aligned}
\delta\left(m, m^{\prime}\right) & =0, \text { if } m \neq m^{\prime}, \text { and } \\
& =1, \text { if } m=m^{\prime} .
\end{aligned}
$$

As a result of Eqs. (3.63-3.65), we find

$$
\begin{aligned}
& -\frac{1}{\alpha_{G \text { Geff }}^{2}} \frac{\partial \alpha_{G_{\text {Geff }}}}{\partial \bar{\rho}_{\mathrm{m}}} \sum_{\mathrm{n}} \frac{\partial \mathrm{p}_{\mathrm{n}}}{\partial \rho_{\mathrm{n}}} \bar{\rho}_{\mathrm{n}}=\frac{\partial \mathrm{p}_{\mathrm{m}^{\prime}}}{\partial \rho_{\mathrm{m}^{\prime}}}\left[\frac{1}{\alpha_{\mathrm{m}^{\prime}}} \delta\left(\mathrm{m}, \mathrm{m}^{\prime}\right)-\frac{\bar{\rho}_{\mathrm{m}^{\prime}}}{\alpha_{\mathrm{m}^{\prime}}^{2}} \frac{\partial \alpha_{\mathrm{m}^{\prime}}}{\partial \bar{\rho}_{\mathrm{m}}}\right] \text {, or rearranging } \\
& \frac{\partial \alpha_{\mathrm{m}^{\prime}}}{\partial \bar{\rho}_{\mathrm{m}}}=\frac{\alpha_{\mathrm{m}^{\prime}}^{2}}{\bar{\rho}_{\mathrm{m}^{\prime}}}\left(\frac{1}{\alpha_{\mathrm{m}^{\prime}}} \delta\left(\mathrm{m}, \mathrm{m}^{\prime}\right)+\frac{\frac{1}{\alpha_{\mathrm{Geff}}^{2}} \frac{\partial \alpha_{\mathrm{Geff}}}{\partial \bar{\rho}_{\mathrm{m}}} \sum_{\mathrm{n}} \frac{\partial \mathrm{p}_{\mathrm{n}}}{\partial \rho_{\mathrm{n}}} \bar{\rho}_{\mathrm{n}}}{\frac{\partial \mathrm{p}_{\mathrm{m}^{\prime}}}{\partial \rho_{\mathrm{m}^{\prime}}}}\right)
\end{aligned}
$$

The complete list $\mathrm{cf}$ the $\alpha_{\mathrm{m}}$ derivatives is given in Table III.3, with the subscripts $n, m, m$ ', and $k$ defined in Table III.1. 
TABLE III.3

TWO-PHASE SITUATIONS: PARTICLE AND LIQUID VOLUME FRACTION DERIVATIVES

$$
\begin{aligned}
& \frac{\partial \alpha_{m^{\prime}}}{\partial \bar{\rho}_{m}}=\frac{\alpha_{m^{\prime}}^{2}}{\bar{\rho}_{m^{\prime}}}\left\{\frac{1}{\alpha_{m^{\prime}}} \delta\left(m, m^{\prime}\right)+\frac{\frac{1}{\alpha_{G e f f}^{2}} \frac{\partial \alpha_{G_{e f f}}}{\partial \bar{\rho}_{m}} \sum \frac{\partial p_{n^{\prime}}}{\partial \rho_{n^{\prime}}} \bar{\rho}_{n^{\prime}}}{\frac{\partial p_{m^{\prime}}}{\partial \rho_{m^{\prime}}}}\right\} \\
& \frac{\partial \alpha_{m^{\prime}}}{\partial \bar{\rho}_{n}}=\frac{\alpha_{m^{\prime}}^{2}}{\bar{\rho}_{m^{\prime}}} \frac{\left\{\frac{-1}{\alpha_{\text {Geff }}} \frac{\partial p_{n}}{\partial \rho_{n}}+\frac{1}{\alpha_{\text {Geff }}^{2}} \frac{\partial \alpha_{\text {Geff }}}{\partial \bar{\rho}_{n}} \sum \frac{\partial p_{n^{\prime}}}{\partial \rho_{n^{\prime}}} \bar{\rho}_{n^{\prime}}\right\}}{\frac{\partial p_{m^{\prime}}}{\partial \rho_{m^{\prime}}}} \\
& \frac{\partial \alpha_{m^{\prime}}}{\partial T_{m}}=\frac{\alpha_{m^{\prime}}^{2}}{\bar{\rho}_{m^{\prime}}} \frac{\left\{\frac{\partial p_{m^{\prime}}}{\partial T_{m^{\prime}}} \delta\left(m, m^{\prime}\right)+\frac{1}{\alpha_{G e f f}^{2}} \frac{\partial \alpha_{\text {Geff }}}{\partial T_{m}} \sum \frac{\partial p_{n^{\prime}}}{\partial \rho_{n^{\prime}}} \bar{p}_{n^{\prime}}\right\}}{\frac{\partial p_{m^{\prime}}}{\partial \rho_{m^{\prime}}}} \\
& \frac{\partial \alpha_{m^{\prime}}}{\partial T_{g}}=\frac{\alpha_{m^{\prime}}^{2}}{\bar{\rho}_{m^{\prime}}} \frac{\left\{\frac{1}{\alpha_{G e f f}^{2}} \frac{\partial \alpha_{G e f f}}{\partial T_{g}} \frac{\sum}{n^{\prime}} \frac{\partial p_{n^{\prime}}}{\partial \rho_{n^{\prime}}} \bar{\rho}_{n^{\prime}}-\sum \frac{\partial p_{n^{\prime}}}{\partial T_{g}}\right\}}{\frac{\partial p_{m^{\prime}}}{\partial \rho_{m^{\prime}}}} \\
& \frac{\partial \alpha_{k}}{\partial \bar{\rho}_{m}}=\frac{\partial \alpha_{k}}{\partial \bar{\rho}_{n}}=\frac{\partial \alpha_{k}}{\partial T_{m}}=\frac{\partial \alpha_{k}}{\partial T_{g}}=0 \\
& \frac{\partial \alpha_{m^{\prime}}}{\partial \bar{\rho}_{k}}=\frac{\partial \alpha_{m^{\prime}}}{\partial T_{k}}=0 \\
& \frac{\partial \alpha_{k}}{\partial \bar{\rho}_{k}}=\frac{\partial \alpha_{k}}{\partial T_{k}}=0
\end{aligned}
$$

III-62 


\section{b. Single-Phase Calculation}

\section{Gas Components}

Two possible configurations are to be examined: either liquid single phase or gas single phase. In both cases, there are no derivatives of the gas volume fraction because the gas volume fraction is constant in any case and equal to $\left(1-\alpha_{s}\right) \alpha_{0}$ for the liquid single-phase configurations or $\left(1-\alpha_{s}\right)$ for the gas single-phase configurations.

\section{Particle and Liquid Components}

As with the two-phase calculation, the pressure equilibrium is used to obtain the volume fraction derivatives, but the solution is quite different, depending on the number of components that take part in this equilibrium.

\section{Three Components}

In this case, the particle and liquid 1 and 2 components take part together in the pressure equilibrium. In this section, the calculation of $\partial \alpha_{m} / \partial \bar{\rho}_{P}(m=P, L 1, L 2)$ is given as an example; the complete results are given in Table III.4. First, we have

$$
\begin{aligned}
& \operatorname{pl}_{L 1}\left(\rho_{L 1}, T_{L 1}\right)=p_{L 2}\left(\rho_{L 2}, T_{L 2}\right), \text { and } \\
& \operatorname{pl}_{L 1}\left(\rho_{L 1}, T_{L 1}\right)=\operatorname{pP}\left(\rho_{P}, T_{P}\right)
\end{aligned}
$$

The pressure equilibrium [Eq. (3.66)] implies the following equations for the derivatives:

$$
\begin{aligned}
& \frac{\partial p_{L 1}}{\partial \rho_{L 1}} \frac{\partial \rho_{L 1}}{\partial \bar{\rho}_{\mathrm{P}}}=\frac{\partial p_{L 2}}{\partial \rho_{\mathrm{L} 2}} \frac{\partial \rho_{\mathrm{L} 2}}{\partial \bar{\rho}_{\mathrm{P}}}, \text { and } \\
& \frac{\partial \mathrm{p}_{\mathrm{L} 1}}{\partial \rho_{\mathrm{L} 1}} \frac{\partial \rho_{\mathrm{L} 1}}{\partial \bar{\rho}_{\mathrm{P}}}=\frac{\partial \mathrm{p}_{\mathrm{P}}}{\partial \rho_{\mathrm{P}}} \frac{\partial \rho_{\mathrm{P}}}{\partial \bar{\rho}_{\mathrm{P}}} \text {. In addition, } \\
& 0=\frac{\partial \alpha_{\mathrm{L} 1}}{\partial \bar{\rho}_{\mathrm{P}}}+\frac{\partial \alpha_{\mathrm{L} 2}}{\partial \bar{\rho}_{\mathrm{P}}}+\frac{\partial \alpha_{\mathrm{p}}}{\partial \bar{\rho}_{\mathrm{P}}}
\end{aligned}
$$

The third equation in the system [Eq. (3.67)] shows that for a single-phase configuration with three components, 
TABLE III.4

VOLUME FRACTION DERIVATIVES

SINGLE-PHASE, THREE COMPONENTS

Derivatives with respect to $\overline{\rho_{P}}$

$$
\left|\begin{array}{ccc}
0 & -\frac{\partial p_{L 1}}{\partial \rho_{L 1}} \frac{\bar{\rho}_{\mathrm{L} 1}}{\alpha_{\mathrm{L} 1}^{2}} & \frac{\partial \mathrm{p}_{\mathrm{L} 2}}{\partial \rho_{\mathrm{L} 2}} \frac{\bar{\rho}_{\mathrm{L} 2}}{\alpha_{\mathrm{L} 2}^{2}} \\
\frac{\partial \mathrm{p}_{\mathrm{P}}}{\partial \rho_{\mathrm{P}}} \frac{\bar{\rho}_{\mathrm{P}}}{\alpha_{\mathrm{P}}^{2}} & -\frac{\partial \mathrm{p}_{\mathrm{L} 1}}{\partial \bar{\rho}_{\mathrm{L} 1}} \frac{\bar{\alpha}_{\mathrm{L} 1}^{2}}{\alpha^{2}} & 0 \\
1 & 1 & 1
\end{array}\right|\left|\begin{array}{c}
\frac{\partial \alpha_{\mathrm{p}}}{\partial \bar{\rho}_{\mathrm{P}}} \\
\frac{\partial \alpha_{\mathrm{L} 1}}{\partial \bar{\rho}_{\mathrm{P}}} \\
\frac{\partial \alpha_{\mathrm{L} 2}}{\partial \bar{\rho}_{\mathrm{P}}}
\end{array}\right|=\left|\begin{array}{c}
0 \\
\frac{\partial p_{\mathrm{p}}}{\partial \rho_{\mathrm{P}}} \frac{1}{\alpha_{\mathrm{P}}} \\
0
\end{array}\right|
$$

Direct solution

$$
\begin{aligned}
& \frac{\partial \alpha_{\mathrm{p}}}{\partial \bar{\rho}_{\mathrm{P}}}=\frac{\partial p_{\mathrm{P}}}{\partial \rho_{\mathrm{P}}} \frac{1}{\alpha_{\mathrm{P}}} \frac{\left[\frac{\partial p_{\mathrm{L} 1}}{\partial \rho_{\mathrm{L} 1}} \frac{\bar{\rho}_{\mathrm{L} 1}}{\alpha_{\mathrm{L} 1}^{2}}+\frac{\partial p_{\mathrm{L} 2}}{\partial \rho_{\mathrm{L} 2}} \frac{\bar{\rho}_{\mathrm{L} 2}}{\alpha_{\mathrm{L} 2}}\right]}{\mathrm{D}} \\
& \frac{\partial \alpha_{L_{1}}}{\partial \bar{\rho}_{\mathrm{P}}}=-\frac{\frac{\partial p_{\mathrm{P}}}{\partial \rho_{\mathrm{P}}} \frac{1}{\alpha_{\mathrm{P}}} \frac{\partial p_{\mathrm{L}_{2}}}{\partial \rho_{\mathrm{L}_{2}}} \frac{\bar{\rho}_{\mathrm{L} 2}}{\alpha_{\mathrm{L} 2}}}{\mathrm{D}} \\
& \frac{\partial \alpha_{L_{2}}}{\partial \bar{\rho}_{\mathrm{P}}}=-\frac{\frac{\partial p_{\mathrm{P}}}{\partial \rho_{\mathrm{P}}} \frac{1}{\alpha_{\mathrm{P}}} \frac{\partial p_{\mathrm{L}_{1}}}{\partial \rho_{\mathrm{L}_{1}}} \frac{\bar{\rho}_{\mathrm{L} 1}}{\alpha_{\mathrm{L}}}}{\mathrm{D}}
\end{aligned}
$$




\section{TABLE III.4 (CONT.)}

Derivatives with respect to $\bar{\rho}_{\mathrm{L} 1}$

Matrix equation

$$
\begin{aligned}
& \left|\begin{array}{ccc}
0 & -\frac{\partial p_{\mathrm{L} 1}}{\partial \rho_{\mathrm{L} 1}} \frac{\bar{\rho}_{\mathrm{L} 1}}{\alpha_{\mathrm{L} 1}^{2}} & \frac{\partial \mathrm{p}_{\mathrm{L} 2}}{\partial \rho_{\mathrm{L} 2}} \frac{\bar{\rho}_{\mathrm{L} 2}}{\alpha_{\mathrm{L} 2}^{2}} \\
\frac{\partial \mathrm{p}_{\mathrm{P}}}{\partial \rho_{\mathrm{P}}} \frac{\bar{\rho}_{\mathrm{P}}}{\alpha_{\mathrm{p}}^{2}} & -\frac{\partial \mathrm{p}_{\mathrm{L} 1}}{\partial \rho_{\mathrm{L} 1}} \frac{\bar{\rho}_{\mathrm{L} 1}}{\alpha_{\mathrm{L} 1}^{2}} & 0 \\
1 & 1 & 1
\end{array}\right|\left|\begin{array}{c}
\frac{\partial \alpha_{\mathrm{p}}}{\partial \bar{\rho}_{\mathrm{L} 1}} \\
\frac{\partial \alpha_{\mathrm{L} 1}}{\partial \bar{\rho}_{\mathrm{L} 1}} \\
\frac{\partial \alpha_{\mathrm{L} 2}}{\partial \bar{\rho}_{\mathrm{L} 1}}
\end{array}\right|=\left|\begin{array}{c}
-\frac{\partial p_{\mathrm{L} 1}}{\partial \rho_{\mathrm{L} 1}} \frac{1}{\alpha_{\mathrm{L} 1}} \\
-\frac{\partial p_{\mathrm{L} 1}}{\partial \rho_{\mathrm{P}}} \frac{1}{\alpha_{\mathrm{L} 1}} \\
0
\end{array}\right| \\
& \frac{\partial \alpha_{\mathrm{p}}}{\partial \bar{\rho}_{\mathrm{L} 1}}=-\frac{\frac{\partial p_{\mathrm{L} 1}}{\partial \rho_{\mathrm{L} 1}} \frac{1}{\alpha_{\mathrm{L} 1}} \frac{\partial p_{\mathrm{L} 2}}{\partial \dot{\rho}_{\mathrm{L} 2}} \frac{\bar{\rho}_{\mathrm{L} 2}}{\alpha_{\mathrm{L} 2}^{2}}}{\mathrm{D}} \\
& \frac{\partial \alpha_{L 1}}{\partial \bar{\rho}_{L 1}}=\frac{\partial p_{L 1}}{\partial \rho_{L 1}} \frac{1}{\alpha_{L 1}} \frac{\left[\frac{\partial p_{L 2}}{\partial \rho_{L 2}} \frac{\bar{\rho}_{L 2}}{\alpha_{L 2}^{2}} \frac{\partial p_{p}}{\partial \rho_{p}} \frac{\bar{\rho}_{\mathrm{P}}}{\alpha_{\mathrm{p}}^{2}}\right]}{D} \\
& \frac{\partial \alpha_{\mathrm{L} 2}}{\partial \bar{\rho}_{\mathrm{L} 1}}=-\frac{\frac{\partial p_{\mathrm{L} 1}}{\partial \rho_{\mathrm{L} 1}} \frac{1}{\alpha_{\mathrm{L} 1}} \frac{\partial p_{\mathrm{P}}}{\partial \rho_{\mathrm{p}}} \frac{\bar{\rho}_{\mathrm{P}}}{\alpha_{\mathrm{p}}^{2}}}{\mathrm{D}}
\end{aligned}
$$




\section{TABLE III.4 (CONT.)}

Derivatives with respect to $\bar{\rho}_{\mathrm{L} 2}$

Matrix equation

$$
\left|\begin{array}{ccc}
0 & -\frac{\partial p_{\mathrm{L} 1}}{\partial \rho_{\mathrm{L} 1}} \frac{\bar{\rho}_{\mathrm{L} 1}}{\alpha_{\mathrm{L} 1}^{2}} & \frac{\partial p_{\mathrm{L} 2}}{\partial \rho_{\mathrm{L} 2}} \frac{\bar{\rho}_{\mathrm{L} 2}}{\alpha_{\mathrm{L} 2}} \\
\frac{\partial p_{\mathrm{P}}}{\partial \rho_{\mathrm{P}}} \frac{\bar{\rho}_{\mathrm{P}}}{\alpha_{\mathrm{p}}^{2}} & -\frac{\partial p_{\mathrm{L} 1}}{\partial \rho_{\mathrm{L} 1}} \frac{\bar{\rho}_{\mathrm{L} 1}}{\alpha_{\mathrm{L} 1}^{2}} & 0 \\
1 & 1
\end{array}\right|\left|\begin{array}{l}
\frac{\partial \alpha_{\mathrm{p}}}{\partial \bar{\rho}_{\mathrm{L} 2}} \\
\frac{\partial \alpha_{\mathrm{L} 1}}{\partial \bar{\rho}_{\mathrm{L} 2}} \\
\frac{\partial \alpha_{\mathrm{L} 2}}{\partial \bar{\rho}_{\mathrm{L} 2}}
\end{array}\right|=\left|\begin{array}{c}
\frac{\partial p_{\mathrm{L} 2}}{\partial \rho_{\mathrm{L} 2}} \frac{1}{\alpha_{\mathrm{L} 2}} \\
0 \\
0
\end{array}\right|
$$

Direct solution

$$
\begin{aligned}
& \frac{\partial \alpha_{\mathrm{p}}}{\partial \rho_{\mathrm{L} 2}}=\frac{\left[\frac{\partial \mathrm{p}_{\mathrm{L} 2}}{\partial \rho_{\mathrm{L} 2}} \frac{1}{\alpha_{\mathrm{L} 2}} \frac{\partial \mathrm{p}_{\mathrm{L} 1}}{\partial \rho_{\mathrm{L} 1}} \frac{\bar{\rho}_{\mathrm{L} 1}}{\alpha_{\mathrm{L} 1}^{2}}\right]}{\mathrm{D}} \\
& \frac{\partial \alpha_{L 1}}{\partial \bar{\rho}_{\mathrm{L} 2}}=-\frac{\frac{\partial p_{\mathrm{L} 2}}{\partial \rho_{\mathrm{L} 2}} \frac{1}{\alpha_{\mathrm{L} 2}} \frac{\partial p_{\mathrm{P}}}{\partial \rho_{\mathrm{P}}} \frac{\bar{\rho}_{\mathrm{P}}}{\alpha_{\mathrm{P}}^{2}}}{\mathrm{D}} \\
& \frac{\partial \alpha_{L 2}}{\partial \bar{\rho}_{L 2}}=\frac{\partial p_{L 2}}{\partial \rho_{L 2}} \frac{1}{\alpha_{L 2}} \frac{\left[\frac{\partial p_{L 1}}{\partial \rho_{L 1}} \frac{\bar{\rho}_{L 1}}{\alpha_{L 1}^{2}}+\frac{\partial p_{p}}{\partial \rho_{p}} \frac{\bar{\rho}_{\mathrm{p}}}{\alpha_{p}^{2}}\right]}{D}
\end{aligned}
$$


TABLE III.4 (CONT.)

Derivatives with respect to $T_{P}$

Matrix equation

$$
\left|\begin{array}{ccc}
0 & -\frac{\partial p_{L 1}}{\partial \rho_{\mathrm{L} 1}} \frac{\bar{\rho}_{\mathrm{L} 1}}{\alpha_{\mathrm{L} 1}} \frac{\partial \mathrm{p}_{\mathrm{L} 2}}{\partial \rho_{\mathrm{L} 2}} \frac{\bar{\rho}_{\mathrm{L} 2}}{\alpha_{\mathrm{L} 2}^{2}} \\
\frac{\partial \mathrm{p}_{\mathrm{P}}}{\partial \rho_{\mathrm{P}}} \frac{\bar{\rho}_{\mathrm{P}}}{\alpha_{\mathrm{P}}^{2}} & -\frac{\partial \mathrm{p}_{\mathrm{L} 1}}{\partial \rho_{\mathrm{L} 1}} \frac{\bar{\rho}_{\mathrm{L} 1}}{\alpha_{\mathrm{L} 1}^{2}} & 0 \\
1 & 1
\end{array}\right|\left|\begin{array}{c}
\frac{\partial \alpha_{\mathrm{p}}}{\partial \mathrm{T}_{\mathrm{P}}} \\
\frac{\partial \alpha_{\mathrm{L} 1}}{\partial \mathrm{T}_{\mathrm{P}}} \\
\frac{\partial \alpha_{\mathrm{L} 2}}{\partial \mathrm{T}_{\mathrm{P}}}
\end{array}\right|=\left|\begin{array}{c}
0 \\
0
\end{array}\right|
$$

Direct solution

$$
\begin{aligned}
& \frac{\partial \alpha_{p}}{\partial T_{p}}=\frac{\partial p_{p}}{\partial T_{p}} \frac{\left[\frac{\partial p_{L 1}}{\partial T_{L 1}} \frac{\bar{\rho}_{L 1}}{\alpha_{L 1}}+\frac{\partial P_{L 2}}{\partial \rho_{L 2}} \frac{\bar{\rho}_{L 2}}{\alpha_{L 2}^{2}}\right]}{D} \\
& \frac{\partial \alpha_{L_{1}}}{\partial \mathrm{T}_{\mathrm{P}}}=-\frac{\left[\frac{\partial \mathrm{p}_{\mathrm{p}}}{\partial \mathrm{T}_{\mathrm{P}}} \frac{\partial \mathrm{p}_{\mathrm{L} 2}}{\partial \rho_{\mathrm{L} 2}} \frac{\bar{\rho}_{\mathrm{L} 2}}{\alpha_{\mathrm{L} 2}^{2}}\right]}{\mathrm{D}} \\
& \frac{\partial \alpha_{L 2}}{\partial T_{P}}=-\frac{\frac{\partial p_{P}}{\partial T_{L 1}} \frac{\partial p_{L 1}}{\partial \rho_{L 1}} \frac{\bar{\rho}_{L 1}}{\alpha_{L 1}}}{D}
\end{aligned}
$$




\section{TABLE III.4 (CONT.)}

Derivatives with respect to $T_{L 1}$

Matrix equation

$$
\left|\begin{array}{ccc}
0 & -\frac{\partial p_{L 1}}{\partial \rho_{L 1}} \frac{\bar{\rho}_{L 1}}{\alpha_{L 1}^{2}} & \frac{\partial p_{L 2}}{\partial \rho_{L 2}} \frac{\bar{\rho}_{L 2}}{\alpha_{L 2}^{2}} \\
\frac{\partial p_{\mathrm{P}}}{\partial \rho_{\mathrm{P}}} \frac{\bar{\rho}_{\mathrm{p}}^{2}}{\alpha_{\mathrm{p}}} & -\frac{\partial \mathrm{p}_{\mathrm{L} 1}}{\partial \rho_{\mathrm{L} 1}} \frac{\bar{\rho}_{\mathrm{L} 1}}{\alpha_{\mathrm{L} 1}^{2}} & 0 \\
1 & 1 & 1
\end{array}\right|\left|\begin{array}{l}
\frac{\partial \alpha_{\mathrm{p}}}{\partial \mathrm{T}_{\mathrm{L} 1}} \\
\frac{\partial \alpha_{\mathrm{L} 1}}{\partial \mathrm{T}_{\mathrm{L} 1}} \\
\frac{\partial \alpha_{\mathrm{L} 2}}{\partial \mathrm{T}_{\mathrm{L} 1}}
\end{array}\right|=\left|\begin{array}{c}
-\frac{\partial \mathrm{p}_{\mathrm{L} 1}}{\partial \mathrm{T}_{\mathrm{L} 1}} \\
-\frac{\partial \mathrm{p}_{\mathrm{L} 1}}{\partial \mathrm{T}_{\mathrm{L} 1}} \\
0
\end{array}\right|
$$

Direct solution

$$
\begin{aligned}
& \frac{\partial \alpha_{\mathrm{p}}}{\partial \mathrm{T}_{\mathrm{L} 1}}=\frac{\left[\frac{\partial \mathrm{p}_{\mathrm{L} 1}}{\partial \mathrm{T}_{\mathrm{L} 1}} \frac{\partial \mathrm{p}_{\mathrm{L} 2}}{\partial \rho_{\mathrm{L} 2}} \frac{\bar{\rho}_{\mathrm{L} 2}}{\alpha_{\mathrm{L} 2}}\right]}{\mathrm{D}} \\
& \frac{\partial \alpha_{L 1}}{\partial T_{L 1}}=\frac{\partial p_{L 1}}{\partial T_{L 1}} \frac{\left[\frac{\partial p_{L 2}}{\partial \rho_{L 2}} \frac{\bar{\rho}_{L 2}}{\alpha_{L 2}^{2}}+\frac{\partial P_{\mathrm{P}}}{\partial \rho_{\mathrm{P}}} \frac{\bar{\rho}_{\mathrm{p}}}{\alpha_{\mathrm{p}}^{2}}\right]}{\mathrm{D}} \\
& \frac{\partial \alpha_{L 2}}{\partial T_{L 1}}=-\frac{\frac{\partial \mathrm{p}_{\mathrm{L} 1}}{\partial \mathrm{T}_{\mathrm{L} 1}} \frac{\partial \mathrm{p}_{\mathrm{p}}}{\partial \rho_{\mathrm{P}}} \frac{\bar{\rho}_{\mathrm{p}}}{\alpha_{\mathrm{P}}^{2}}}{\mathrm{D}}
\end{aligned}
$$




\section{TABLE III.4 (CONT.)}

Derivatives with respect to $T_{L 2}$ Matrix equation

$$
\left|\begin{array}{ccc}
0 & -\frac{\partial p_{\mathrm{L}}}{\partial \rho_{\mathrm{L} 1}} \frac{\bar{\rho}_{\mathrm{L} 1}}{\alpha_{\mathrm{L} 1}^{2}} & \frac{\partial \mathrm{p}_{\mathrm{L} 2}}{\partial \rho_{\mathrm{L} 2}} \frac{\bar{\rho}_{\mathrm{L} 2}}{\alpha_{\mathrm{L} 2}^{2}} \\
\frac{\partial \mathrm{p}_{\mathrm{P}}}{\partial \rho_{\mathrm{P}}} \frac{\bar{\rho}_{\mathrm{P}}}{\alpha_{\mathrm{P}}^{2}} & -\frac{\partial \mathrm{p}_{\mathrm{L} 1}}{\partial \rho_{\mathrm{L} 1}} \frac{\bar{\rho}_{\mathrm{L} 1}}{\alpha_{\mathrm{L} 1}^{2}} & 0 \\
1 & 1 & 1
\end{array}\right|\left|\begin{array}{c}
\frac{\partial \alpha_{\mathrm{p}}}{\partial \mathrm{T}_{\mathrm{L} 2}} \\
\frac{\partial \alpha_{\mathrm{L} 1}}{\partial \mathrm{T}_{\mathrm{L} 2}} \\
\frac{\partial \alpha_{\mathrm{L} 2}}{\partial \mathrm{T}_{\mathrm{L} 2}}
\end{array}\right|=\left|\begin{array}{c}
\frac{\partial \mathrm{p}_{\mathrm{L} 2}}{\partial \mathrm{T}_{\mathrm{L} 2}} \\
0 \\
0
\end{array}\right|
$$

Direct solution

$$
\begin{aligned}
& \frac{\partial \alpha_{\mathrm{p}}}{\partial \mathrm{T}_{\mathrm{L} 2}}=-\frac{\frac{\partial \mathrm{p}_{\mathrm{L} 2}}{\partial \mathrm{T}_{\mathrm{L}}} \frac{\partial \mathrm{p}_{\mathrm{L} 1}}{\partial \rho_{\mathrm{L} 1}} \frac{\bar{\rho}_{\mathrm{LI}}}{\alpha_{\mathrm{L} 1}}}{\mathrm{D}} \\
& \frac{\partial \alpha_{L 1}}{\partial T_{L 2}}=-\frac{\frac{\partial p_{L 2}}{\partial T_{L 2}} \frac{\partial p_{p}}{\partial \rho_{p}} \frac{\bar{\rho}_{p}}{\alpha_{p}^{2}}}{D} \\
& \frac{\partial \alpha_{L 2}}{\partial \mathrm{T}_{\mathrm{L} 2}}=\frac{\partial \mathrm{p}_{\mathrm{L} 2}}{\partial \mathrm{T}_{\mathrm{L} 2}} \frac{\left[\frac{\partial \mathrm{p}_{\mathrm{L} 1}}{\partial \rho_{\mathrm{L} 1}} \frac{\bar{\rho}_{\mathrm{L} 1}}{\alpha_{\mathrm{L} 1}^{2}}+\frac{\partial \mathrm{p}_{\mathrm{p}}}{\partial \rho_{\mathrm{p}}} \frac{\bar{\rho}_{\mathrm{p}}}{\alpha_{\mathrm{p}}^{2}}\right]}{\mathrm{D}}
\end{aligned}
$$




\section{TABLE III.4 (CONT.)}

With the denominator $D$ equal to

$$
\begin{aligned}
& \mathrm{D}=\frac{\partial \mathrm{p}_{\mathrm{p}}}{\partial \rho_{\mathrm{p}}} \frac{\bar{\rho}_{\mathrm{p}}}{\alpha_{\mathrm{p}}^{2}} \frac{\partial \mathrm{p}_{\mathrm{L} 1}}{\partial \rho_{\mathrm{L} 1}} \frac{\bar{\rho}_{\mathrm{L} 1}}{\alpha_{\mathrm{L} 1}^{2}}+\frac{\partial \mathrm{p}_{\mathrm{L} 1}}{\partial \rho_{\mathrm{L} 1}} \frac{\bar{\rho}_{\mathrm{L} 1}}{\alpha_{\mathrm{L} 1}^{2}} \frac{\partial \mathrm{p}_{\mathrm{L} 2}}{\partial \rho_{\mathrm{L} 2}} \frac{\bar{\rho}_{\mathrm{L} 2}}{\alpha_{\mathrm{L} 2}^{2}}+\frac{\partial \mathrm{p}_{\mathrm{L} 2}}{\partial \rho_{\mathrm{L} 2}} \frac{\bar{\rho}_{\mathrm{L} 2}}{\alpha_{\mathrm{L} 2}} \frac{\partial \mathrm{p}_{\mathrm{p}}}{\partial \rho_{\mathrm{p}}} \frac{\bar{\rho}_{\mathrm{p}}}{\alpha_{\mathrm{p}}^{2}} \text {, and } \\
& \frac{\partial \alpha_{m}}{\partial \bar{\rho}_{n}}=0 \\
& \mathrm{~m}=\mathrm{P}, \mathrm{L} 1, \mathrm{~L} 2 \\
& \mathrm{n}=\mathrm{g} 1, \mathrm{~g} 2, \mathrm{~g} 3 \\
& \frac{\partial \alpha_{\mathrm{m}}}{\partial \mathrm{T}_{\mathrm{g}}}=0 \\
& \sum_{\mathrm{m}} \alpha_{\mathrm{m}} \equiv \text { Const }=1,(\mathrm{~m}=\mathrm{P}, \mathrm{L} 1, \mathrm{~L} 2) .
\end{aligned}
$$

By setting $m^{\prime}$ to $m$ and $m$ to $P$ in Eq. (3.64), we have

$$
\frac{\partial \rho_{m}}{\partial \bar{\rho}_{p}}=\frac{1}{\alpha_{p}}-\delta(m, P)-\frac{\bar{\rho}_{m} \partial \alpha_{m}}{\alpha_{m}^{2} \partial \bar{\rho}_{p}}
$$

Using the previous equation and Eq. (3.67), the solution vector $\partial \alpha_{m} / \partial \bar{\rho} p$ can be obtained by inverting the following matrix equation system: 


$$
\left|\begin{array}{ccc}
0 & -\frac{\partial \mathrm{p}_{\mathrm{L} 1}}{\partial \rho_{\mathrm{L} 1}} \frac{\bar{\rho}_{\mathrm{L} 1}}{\alpha_{\mathrm{L} 1}^{2}} & \frac{\partial \mathrm{p}_{\mathrm{L} 2}}{\partial \rho_{\mathrm{L} 2}} \frac{\bar{\rho}_{\mathrm{L} 2}}{\alpha_{\mathrm{L} 2}^{2}} \\
\frac{\partial \mathrm{p}_{\mathrm{P}}}{\partial \rho_{\mathrm{P}}} \bar{\rho}_{\mathrm{P}} & -\frac{\partial \mathrm{p}_{\mathrm{L} 1}}{\partial \rho_{\mathrm{p}}} \frac{\bar{\rho}_{\mathrm{L} 1}}{\alpha_{\mathrm{L}}^{2}} & 0 \\
1 & 1 & 1
\end{array}\right|\left|\begin{array}{c}
\frac{\partial \alpha_{\mathrm{p}}}{\partial \bar{\rho}_{\mathrm{p}}} \\
\frac{\partial \alpha_{\mathrm{L} 1}}{\partial \bar{\rho}_{\mathrm{p}}} \\
\frac{\partial \alpha_{\mathrm{L} 2}}{\partial \bar{\rho}_{\mathrm{P}}}
\end{array}\right|=\left|\begin{array}{c}
0 \\
\frac{\partial \mathrm{p}_{\mathrm{p}}}{\partial \rho_{\mathrm{p}}} \frac{1}{\alpha_{\mathrm{p}}} \\
0
\end{array}\right|
$$

This matrix equation system can be solved directly to yield

$$
\begin{aligned}
& \frac{\partial \alpha_{\mathrm{p}}}{\partial \bar{\rho}_{\mathrm{p}}}=\frac{\partial \mathrm{p}_{\mathrm{p}}}{\partial \rho_{\mathrm{P}}} \frac{\left[\frac{\partial \mathrm{p}_{\mathrm{L} 1}}{\alpha_{\mathrm{p}}} \frac{\bar{\rho}_{\mathrm{L} 1}}{\partial \rho_{\mathrm{L} 1}}+\frac{\partial \mathrm{p}_{\mathrm{L} 2}}{\partial \rho_{\mathrm{L} 1}} \frac{\bar{\rho}_{\mathrm{L} 2}}{\partial \rho_{\mathrm{L} 2}}\right]}{\mathrm{D}}, \\
& \frac{\partial \alpha_{\mathrm{L} 1}}{\partial \bar{\rho}_{\mathrm{P}}}=-\frac{\frac{\partial \mathrm{p}_{\mathrm{p}}}{\partial \rho_{\mathrm{p}}} \frac{1}{\alpha_{\mathrm{p}}} \frac{\partial \mathrm{p}_{\mathrm{L} 2}}{\partial \rho_{\mathrm{L} 2}} \frac{\bar{\rho}_{\mathrm{L} 2}}{\alpha_{\mathrm{L} 2}^{2}}}{\mathrm{D}} \text {, and } \\
& \frac{\partial \alpha_{\mathrm{L}}}{\partial \bar{\rho}_{\mathrm{P}}}=-\frac{\frac{\partial \mathrm{p}_{\mathrm{p}}}{\partial \rho_{\mathrm{p}}} \frac{\partial}{\alpha_{\mathrm{p}}} \frac{\partial \mathrm{p}_{\mathrm{L} 1}}{\partial \rho_{\mathrm{L} 1}} \frac{\bar{\rho}_{\mathrm{Ll}}}{\alpha_{\mathrm{L} 1}^{2}}}{\mathrm{D}},
\end{aligned}
$$

where

$$
\mathrm{D}=\frac{\partial \mathrm{p}_{\mathrm{p}}}{\partial \rho_{\mathrm{P}}} \frac{\bar{\rho}_{\mathrm{P}}}{\alpha_{\mathrm{p}}^{2}} \frac{\partial p_{\mathrm{L} 1}}{\partial \rho_{\mathrm{L} 1}} \frac{\bar{\rho}_{\mathrm{L} 1}}{\alpha_{\mathrm{L} 1}^{2}}+\frac{\partial p_{\mathrm{L} 1}}{\partial \rho_{\mathrm{L} 1}} \frac{\bar{\rho}_{\mathrm{L} 1}}{\alpha_{\mathrm{L} 1}^{2}} \frac{\partial \mathrm{p}_{\mathrm{L} 2}}{\partial \rho_{\mathrm{L} 2}} \frac{\bar{\rho}_{\mathrm{L} 2}}{\alpha_{\mathrm{L} 2}^{2}}+\frac{\partial p_{\mathrm{L} 2}}{\partial \rho_{\mathrm{L} 2}} \frac{\bar{\rho}_{\mathrm{L} 2}}{\alpha_{\mathrm{L} 2}^{2}} \frac{\partial \mathrm{p}_{\mathrm{p}}}{\partial \rho_{\mathrm{p}}} \frac{\bar{\rho}_{\mathrm{p}}}{\alpha_{\mathrm{p}}^{2}} .
$$

In this configuration, the gas volume fraction is kept arbitrarily constant at a small part $\left(1-\alpha_{s}\right) \alpha_{0}$ of the numerical cell, and all derivatives in which gas components are involved are equal to zero. 
Two Components

In the case where only liquids 1 and 2 are present in a cell, the equation system [Eq. (3.66)] is reduced to one equation because the particles do not take part in the pressure equilibrium:

$$
\begin{aligned}
& p_{L 1}\left(\rho_{L 1}, T_{L 1}\right)=p_{L 2}\left(\rho_{L 2}, T_{L 2}\right), \\
& \frac{\partial p_{L 1}}{\partial \rho_{L 1}} \frac{\partial \rho_{L 1}}{\partial \bar{\rho}_{L 1}}=\frac{\partial p_{L 2}}{\partial \rho_{L 2}} \frac{\partial \rho_{L 2}}{\partial \bar{\rho}_{L 1}} \text {, and } \\
& 0=\frac{\partial \alpha_{L 1}}{\partial \bar{\rho}_{L 1}}+\frac{\partial \alpha_{L 2}}{\partial \bar{\rho}_{L 1}},
\end{aligned}
$$

with

$$
\begin{aligned}
& \frac{\partial \rho_{\mathrm{L1}}}{\partial \bar{\rho}_{\mathrm{L} 1}}=\frac{1}{\alpha_{\mathrm{L} 1}}-\frac{\bar{p}_{\mathrm{L} 1}}{\alpha_{L 1}^{2}} \frac{\partial \alpha_{\mathrm{L} 1}}{\partial \bar{\rho}_{\mathrm{L} 1}} \text {, and } \\
& \frac{\partial \rho_{\mathrm{L} 2}}{\partial \bar{\rho}_{\mathrm{L} 1}}=-\frac{\bar{p}_{\mathrm{L} 2}}{\alpha_{\mathrm{L} 2}^{2}} \frac{\partial \alpha_{\mathrm{L} 2}}{\partial \bar{\rho}_{\mathrm{L} 1}} .
\end{aligned}
$$

The derivatives are obtained by combining Eqs. (3.68) and (3.69).

$$
\begin{aligned}
& \frac{\partial \alpha_{L_{1}}}{\partial \bar{\rho}_{L_{1}}}=\frac{\frac{\partial p_{L 1}}{\partial \rho_{L 1}} \frac{1}{\alpha_{L 1}}}{\left[\frac{\partial p_{L 1}}{\partial \rho_{L 1}} \frac{\bar{\rho}_{L 1}}{\alpha_{L 1}^{2}}+\frac{\partial p_{L 2}}{\partial \rho_{L 2}} \frac{\bar{\rho}_{L 2}}{\alpha_{L 2}^{2}}\right]} \text {, and } \\
& \frac{\partial \alpha_{L 2}}{\partial \bar{\rho}_{L 1}}=\frac{-\frac{\partial p_{L 1}}{\partial \rho_{L 1}} \frac{1}{\alpha_{L 1}}}{\left[\frac{\partial p_{L 1}}{\partial \rho_{L 1}} \frac{\bar{\rho}_{L 1}}{\alpha_{L 1}^{2}}+\frac{\partial p_{L 2}}{\partial \rho_{L 2}} \frac{\bar{\rho}_{L 2}}{\alpha_{L 2}}\right]} .
\end{aligned}
$$

III-72 
Obviously, this is similar to previous sections, where all the volume fraction derivatives in which either the gas components or the liquid missing components are involved are set equal to zero.

For complete results, see Table III.5.

\section{One Component}

Either for the gas or liquid phase, this case is trivial because the volume is constant, and all the derivatives are zero.

\section{Other Derivatives}

\section{a. Pressure Derivatives}

\section{Two Phases}

The variables $\rho$ and $\mathrm{T}$ are independent in the AFDM algorithm. Therefore, the pressure derivatives with respect to microdensity and temperature for every component are given by

$$
\begin{aligned}
& \frac{\partial p}{\partial \bar{\rho}_{m}}=\frac{\partial p_{m}}{\partial \rho_{m}}\left[\frac{1}{\alpha_{m}}-\frac{\bar{\rho}_{m}}{\alpha_{m}^{2}} \frac{\partial \alpha_{m}}{\partial \bar{\rho}_{m}}\right] \\
& \frac{\partial p}{\partial \bar{\rho}_{n}}=\sum_{n^{\prime}} \frac{\partial p_{n^{\prime}}}{\partial \bar{\rho}_{n}}=\frac{\partial p_{n}}{\partial \rho_{n}} \frac{1}{\alpha_{G e f f}}-\frac{1}{\alpha_{G e f f}^{2}} \frac{\partial \alpha_{\text {Geff }}}{\partial \bar{\rho}_{n}} \sum_{n^{\prime}} \frac{\partial p_{n^{\prime}}}{\partial \rho_{n^{\prime}}} \bar{\rho}_{n^{\prime}}, \\
& \frac{\partial p}{\partial T_{m}}=\frac{\partial p_{m}}{\partial T_{m}}-\frac{\bar{\rho}_{m}}{\alpha_{m}^{2}} \frac{\partial p_{m}}{\partial p_{m}} \frac{\partial \alpha_{m}}{\partial T_{m}} \text {, and } \\
& \frac{\partial p}{\partial T_{g}}=\sum_{n^{\prime}} \frac{\partial p_{n^{\prime}}}{\partial T_{g}}+\sum_{n^{\prime}} \frac{\partial p_{n^{\prime}}}{\partial \rho_{n^{\prime}}} \frac{\partial \rho_{n^{\prime}}}{\partial T_{g}}=\sum_{n^{\prime}} \frac{\partial \rho_{n^{\prime}}}{\partial T_{g}}-\frac{1}{\alpha_{G^{\prime}}^{2}} \frac{\partial \alpha_{G_{\text {Geff }}}}{\partial T_{g}} \sum_{n^{\prime}} \frac{\partial p_{n^{\prime}}}{\partial \rho_{n^{\prime}}} \bar{\rho}_{n^{\prime}}
\end{aligned}
$$

For the missing components $\mathrm{k}$ :

$$
\frac{\partial p}{\partial \bar{p}_{k}}=0 \quad \frac{\partial p}{\partial T_{k}}=0
$$

where the subscripts $m, n^{\prime}$, and $k$ are defined in Table III.1, and the derivatives of the volume fractions are defined in Tables III. 2 and III.3. 
TABLE III.5

SINGLE-PHASE: TWO COMPONENTS VOLUME FRACTION DERIVATIVES

\begin{tabular}{l}
\hline $\mathrm{m}=\mathrm{P}, \mathrm{LI}$ \\
$\mathrm{m}^{\prime}=\mathrm{P}, \mathrm{L} 1$ \\
\hline missing component: \\
$\mathrm{k}=\mathrm{L} 2$
\end{tabular}

\begin{tabular}{|c|}
\hline $\begin{aligned} \mathrm{m} & =\mathrm{P}, \mathrm{L} 2 \\
\mathrm{~m}^{\prime} & =\mathrm{P}, \mathrm{L} 2\end{aligned}$ \\
\hline $\begin{array}{l}\text { missing component: } \\
\mathrm{k}=\mathrm{L} 1\end{array}$ \\
\hline
\end{tabular}

$\begin{aligned} m & =\mathrm{L} 1, \mathrm{~L} 2 \\ \mathrm{~m}^{\prime} & =\mathrm{L} 1, \mathrm{~L} 2\end{aligned}$

missing component:

$\mathbf{k}=\mathbf{P}$

$$
\begin{aligned}
& \frac{\partial \alpha_{m}}{\partial \bar{\rho}_{m^{\prime}}}=\frac{\left[1-2\left(1-\delta\left(m, m^{\prime}\right)\right\}\right] \frac{\partial p_{m^{\prime}}}{\partial \rho_{m^{\prime}}} \frac{1}{\alpha_{m^{\prime}}}}{\sum_{m} \frac{\partial p_{m}}{\partial \rho_{m}} \frac{\bar{\rho}_{m}}{\alpha_{m}^{2}}} \\
& \frac{\partial \alpha_{m}}{\partial \mathrm{T}_{\mathrm{m}^{\prime}}}=\frac{\left[1-2\left\{1-\delta\left(\mathrm{m}, \mathrm{m}^{\prime}\right)\right\}\right]}{\sum_{\mathrm{m}} \frac{\partial \mathrm{p}_{\mathrm{m}}}{\partial \rho_{\mathrm{m}}} \frac{\bar{\rho}_{\mathrm{m}}}{\alpha_{\mathrm{m}}^{2}}} \\
& \begin{array}{l}
\frac{\partial \alpha_{m}}{\partial \bar{\rho}_{n}}=0 \\
\frac{\partial \alpha_{m}}{\partial T_{g}}=0
\end{array} \mid n=g 1, g 2, g 3 \\
& \frac{\partial \alpha_{k}}{\partial \bar{\rho}_{m}}=0 \quad \frac{\partial \alpha_{k}}{\partial T_{m}}=0 \\
& \frac{\partial \alpha_{m}}{\partial \bar{\rho}_{k}}=0 \quad \frac{\partial \alpha_{m}}{\partial T_{k}}=0 \\
& \frac{\partial \alpha_{k}}{\partial \bar{\rho}_{k}}=0 \quad \frac{\partial \alpha_{k}}{\partial T_{k}}=0
\end{aligned}
$$




\section{Single Phase \\ Two or Three Components}

The terms $\partial \mathrm{p} / \partial \bar{\rho}_{\mathrm{m}}$ and $\partial \mathrm{p} / \partial \mathrm{T}_{\mathrm{m}}$ must be obtained by using Eqs. (3.70) and (3.72), with the same conditions for the missing components as in the preceding section. The subscripts $\mathrm{m}$ and $\mathrm{k}$ and the volume fraction derivatives are defined in Tables III.4 and III.5.

$$
\begin{array}{ll}
\frac{\partial p}{\partial \bar{\rho}_{n}}=0 & \frac{\partial p}{\partial \tilde{r}_{\mathrm{g}}}=0 \text { and } \\
\frac{\partial \mathrm{p}}{\partial \bar{\rho}_{\mathrm{k}}}=0 & \frac{\partial \mathrm{p}}{\partial \mathrm{T}_{\mathrm{k}}}=0 .
\end{array}
$$

\section{One Component}

In the case of one liquid component, $\partial \mathrm{p} / \partial \bar{\rho}_{\mathrm{m}}$ and $\partial \mathrm{p} / \partial \mathrm{T}_{\mathrm{m}}$ are calculated by using Eqs. (3.70) and (3.72) with volume fraction derivatives, as quoted in Sec. 3.b.

$$
\begin{array}{ll}
\frac{\partial p}{\partial \bar{\rho}_{n}}=0 & \frac{\partial p}{\partial T_{g}}=0 \quad \text { and } \\
\frac{\partial p}{\partial \bar{\rho}_{k}}=0 & \frac{\partial p}{\partial T_{k}}=0 .
\end{array}
$$

In the case of one gas component,

$$
\frac{\partial p}{\partial \bar{\rho}_{m}}=0 \quad \frac{\partial p}{\partial T_{m}}=0
$$

and $\partial \mathrm{p} / \partial \bar{\rho}_{\mathrm{n}}$ and $\partial \mathrm{p} / \partial \mathrm{T}_{\mathrm{g}}$ are calculated by using Eqs. (3.71) and (3.73):

$$
\frac{\partial p}{\partial \bar{\rho}_{k}}=0 \quad \frac{\partial p}{\partial T_{k}}=0
$$


b. Particle and Liquid Internal Energy Derivatives

\section{Two Phases}

The derivatives may be written as

$$
\begin{aligned}
& \frac{\partial e_{m}}{\partial \bar{\rho}_{m^{\prime}}}=\frac{\partial e_{m}}{\partial \rho_{m}} \frac{\partial \rho_{m}}{\partial \bar{\rho}_{m^{\prime}}}=\frac{\partial e_{m}}{\partial \rho_{m}}\left[\delta\left(m, m^{\prime}\right) \frac{1}{\alpha_{m}}-\frac{\bar{\rho}_{m}}{\alpha_{m}^{2}} \frac{\partial \alpha_{m^{\prime}}}{\partial \bar{\rho}_{m^{\prime}}}\right], \\
& \frac{\partial e_{m}}{\partial \bar{\rho}_{n}}=-\frac{\partial e_{m}}{\partial \rho_{m}} \frac{\bar{\rho}_{m}}{\alpha_{m^{\prime}}^{2}} \frac{\partial \alpha_{m}}{\partial \bar{\rho}_{n}}, \\
& \frac{\partial e_{m}}{\partial T_{m^{\prime}}}=\delta\left(m, m^{\prime}\right) \frac{\partial e_{m}}{\partial T_{m^{\prime}}}-\frac{\partial e_{m}}{\partial \rho_{m}} \frac{\bar{\rho}_{m}}{\alpha_{m^{\prime}}} \frac{\partial \alpha_{m}}{\partial T_{m^{\prime}}}, \text { and } \\
& \frac{\partial e_{m}}{\partial T_{g}}=-\frac{\partial e_{m}}{\partial \rho_{m}} \frac{\bar{\rho}_{m}}{\alpha_{m}^{2}} \frac{\partial \alpha_{m}}{\partial T_{g}} .
\end{aligned}
$$

\section{Missing Components}

$$
\begin{aligned}
& \frac{\partial e_{m}}{\partial \bar{\rho}_{k}}=0 \quad \frac{\partial e_{k}}{\partial \bar{\rho}_{m}}=0, \\
& \frac{\partial e_{m}}{\partial T_{k}}=0 \quad \frac{\partial e_{k}}{\partial T_{m}}=0, \text { and } \\
& \frac{\partial e_{k}}{\partial T_{k}}=\left(\frac{\partial e_{k}}{\partial T_{k}}\right)_{S a t} .
\end{aligned}
$$

The subscripts $m, \mathrm{~m}^{\prime}$, and $\mathrm{k}$ are defined in Table III.1, and the volume fraction derivatives are defined in Tables III.2 and III.3. 


\section{Single Phase}

Equations (3.74)-(3.77) may be used with volume fraction derivatives defined in Table MI.4 for three component configurations or Table III.5 in the case of one or several missing components. The subscripts $\mathrm{m}, \mathrm{m}$, and $\mathrm{k}$ are defined in Table III.5 for the configurations in which a component is missing.

c. Gas Internal Energy Derivatives

\section{Two Phases \\ Total Energy}

The total internal energy in the gas is equal to

$$
e_{g}=\sum_{n} e_{n} \frac{\rho_{n}}{\rho_{g}}
$$

Therefore,

$$
\begin{aligned}
& \frac{\partial e_{g}}{\partial \bar{\rho}_{m}}=\sum_{n}\left\{\frac{\partial\left(\frac{\bar{\rho}_{n}}{\bar{\rho}_{g}}\right)}{\partial \bar{\rho}_{m}} e_{n}+\frac{\bar{\rho}_{n}}{\bar{\rho}_{g}} \frac{\partial e_{n}}{\partial \bar{\rho}_{m}}\right\} \text {, and } \\
& \frac{\partial\left(\frac{\bar{\rho}_{n}}{\bar{\rho}_{g}}\right)}{\partial \bar{\rho}_{m}}=\frac{1}{\rho_{g}} \frac{\partial \rho_{n}}{\partial \bar{\rho}_{m}}-\frac{\rho_{n}}{\rho_{g}^{2}} \sum_{n} \frac{\partial \rho_{n}}{\partial \bar{\rho}_{m}} .
\end{aligned}
$$

By using the three previous equations, after some algebra, the total gas internal energy may be written as

$$
\frac{\partial \mathrm{e}_{\mathrm{g}}}{\partial \bar{\rho}_{\mathrm{m}}}=-\frac{1}{\alpha_{\text {Geff }}^{2}} \frac{\partial \alpha_{\text {Geff }}}{\partial \bar{\rho}_{\mathrm{m}}} \sum_{\mathrm{n}} \frac{\bar{\rho}_{\mathrm{n}}^{2}}{\bar{\rho}_{\mathrm{g}}} \frac{\partial \mathrm{e}_{\mathrm{n}}}{\partial \rho_{\mathrm{n}}} .
$$

The other derivatives are calculated accordingly:

$$
\frac{\partial e_{g}}{\partial \bar{\rho}_{n^{\prime}}}=-\frac{e_{n^{\prime}}-e_{g}}{\bar{\rho}_{g}}+\frac{\rho_{n^{\prime}}}{\bar{\rho}_{g}} \frac{\partial e_{n^{\prime}}}{\partial \rho_{n^{\prime}}}-\frac{1}{\alpha_{G e f f}^{2}} \frac{\partial \alpha_{G_{\text {Geff }}}}{\partial \bar{\rho}_{n^{\prime}}} \sum_{n} \frac{\bar{\rho}_{n}^{2}}{\bar{\rho}_{g}} \frac{\partial e_{n}}{\partial \rho_{n}}
$$




$$
\begin{aligned}
& \frac{\partial e_{g}}{\partial T_{m}}=-\frac{1}{\alpha_{G e f f}^{2}} \frac{\partial \alpha_{G e f f}}{\partial T_{m}} \sum_{n} \frac{\bar{\rho}_{n}^{2}}{\bar{\rho}_{g}} \frac{\partial e_{n}}{\partial \rho_{n}} \text {, and } \\
& \frac{\partial e_{g}}{\partial T_{g}}=-\frac{1}{\alpha_{G e f f}^{2}} \frac{\partial \alpha_{G e f f}}{\partial T_{g}} \sum_{n} \frac{\bar{\rho}_{n}^{2}}{\bar{\rho}_{g}} \frac{\partial e_{n}}{\partial \rho_{n}}+\sum_{n} \frac{\rho_{n}}{\rho_{g}} \frac{\partial e_{n}}{\partial T_{g}} .
\end{aligned}
$$

The effective gas volume fraction derivatives are defined in Table III.2.

\section{Partial Energies}

The partial gas energy derivatives are calculated in a simpler way:

$$
\begin{aligned}
& \frac{\partial e_{n}}{\partial \bar{\rho}_{n^{\prime}}}=\frac{\partial e_{n}}{\partial \rho_{n}}\left[\delta\left(n, n^{\prime}\right) \frac{1}{\alpha_{G e f f}}-\frac{\bar{\rho}_{n}}{\alpha_{G e f f}^{2}} \frac{\partial \alpha_{\text {Geff }}}{\partial \bar{\rho}_{n^{\prime}}}\right] \quad \delta\left(n, n^{\prime}\right)=\left\{\begin{array}{l}
1, \text { if } n=n^{\prime} \\
0, \text { if } n \neq n^{\prime}
\end{array}\right. \\
& \frac{\partial e_{n}}{\partial T_{g}}=\frac{\partial e_{H}}{\partial T_{g}}-\frac{\partial e_{n}}{\partial \rho_{n}}\left[\frac{\bar{\rho}_{n}}{\alpha_{G e f f}^{2}} \frac{\partial \alpha_{G e f f}}{\partial T_{g}}\right] \quad \begin{array}{c}
n=g 1, g 2, g 3 \\
n^{\prime}=g 1, g 2, g 3
\end{array} \\
& \frac{\partial e_{n}}{\partial \bar{\rho}_{m}}=-\frac{\partial e_{n}}{\partial \rho_{n}}\left[\frac{\bar{\rho}_{n}}{\alpha_{\text {Geff }}^{2}} \frac{\partial \alpha_{\text {Geff }}}{\partial \bar{\rho}_{m}}\right] \quad n=g 1, g 2, g 3 \\
& \frac{\partial e_{n}}{\partial T_{m}}=-\frac{\partial e_{n}}{\partial \rho_{n}}\left[\frac{\bar{\rho}_{n}}{\alpha_{\text {Geff }}^{2}} \frac{\partial \alpha_{\text {Geff }}}{\partial T_{m}}\right] \quad \text { m for liquid components }
\end{aligned}
$$

The volume fraction derivatives are defined in Table III.2.

\section{Single Phase}

Equations (3.78) through (3.81) for total energy derivatives and Eqs. (3.82) and (3.83) for partial gas energy derivatives are still valid because the derivatives of the effective gas volume fractions are equal to zero.

d. Saturation Temperature Derivatives. Because the saturation temperature depends only on the partial pressure of the corresponding gas component, the derivatives are given by 


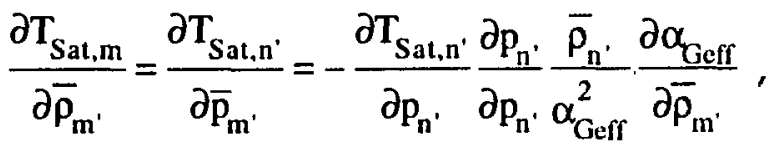

$$
\begin{aligned}
& \frac{\partial \mathrm{T}_{\text {Sat,m }}}{\partial \bar{\rho}_{\mathrm{n}}}=\frac{\partial \mathrm{T}_{\mathrm{Sat}^{\prime} \mathrm{n}^{\prime}}}{\partial \bar{\rho}_{\mathrm{n}}}=\frac{\partial \mathrm{T}_{{\text {Sat }, n^{\prime}}}}{\partial \mathrm{p}_{\mathrm{n}^{\prime}}} \frac{\partial \mathrm{p}_{\mathrm{n}^{\prime}}}{\partial \rho_{\mathrm{n}^{\prime}}}\left[\delta\left(\mathrm{n}, \mathrm{n}^{\prime}\right) \frac{1}{\alpha_{\text {Geff }}} \frac{\bar{\rho}_{\mathrm{n}^{\prime}}}{\alpha_{\text {Geff }}^{2}} \frac{\partial \alpha_{\text {Geff }}}{\partial \bar{\rho}_{\mathrm{n}}}\right] \\
& \frac{\partial T_{\text {Sat, } m}}{\partial T_{m^{\prime}}}=\frac{\partial T_{S_{a t, n^{\prime}}}}{\partial T_{m^{\prime}}}=-\frac{\partial T_{S_{a t, n^{\prime}}}}{\partial p_{n^{\prime}}} \frac{\partial p_{n^{\prime}}}{\partial \rho_{n^{\prime}} \cdot \bar{\rho}_{\mathrm{n}^{\prime}}} \frac{\partial \alpha_{G_{\text {Geff }}}}{\partial T_{m^{\prime}}} \text {, and } \\
& \frac{\partial T_{S a t, m}}{\partial T_{g}}=\frac{\partial T_{S_{a t}, n^{\prime}}}{\partial T_{g}}=\frac{\partial T_{S_{a t, n^{\prime}}}}{\partial p_{n^{\prime}}}\left[\frac{\partial p_{n^{\prime}}}{\partial T_{g}}-\frac{\partial p_{n^{\prime}}}{\partial \rho_{n^{\prime}}, \bar{\rho}_{\mathrm{n}^{\prime}}} \frac{\partial \alpha_{G_{\text {Geff }}}^{2}}{\partial T_{g}}\right],
\end{aligned}
$$

with

$$
\begin{aligned}
\mathrm{m} & =\mathrm{m}^{\prime}=\mathrm{L} 1, \mathrm{~L} 2, \text { and } \\
\mathrm{n} & =\mathrm{g} 1, \mathrm{~g} 2 .
\end{aligned}
$$

If $\mathrm{m}=\mathrm{L} 1$, then $\mathrm{n}^{\prime}=\mathrm{g} 1$;

if $m=L 2$, then $n^{\prime}=g 2$.

The effective gas volume fraction derivatives are given in Table II.2. Because these volume fraction derivatives are equal to zero in the case of single liquid or gas phase, the Eqs. (3.84)-(3.87) are still valid in these configurations.

e. Other Saturation Property Derivatives. If the derivatives of the saturation temperature are known as explained in the above section, it is easy to obtain the following derivatives.

\section{Condensated Liquid Internal Energies}

$$
\frac{\partial \mathrm{e}_{\mathrm{Con}, \mathrm{m}}}{\partial \bar{\rho}_{\mathrm{m}^{\prime}}}=\frac{\partial \mathrm{e}_{\mathrm{Con}, \mathrm{n}^{\prime}}}{\partial \mathrm{T}_{\mathrm{Sat}_{\mathrm{n}} \mathrm{n}^{\prime}}} \frac{\partial \mathrm{T}_{\mathrm{Sat}_{\mathrm{n}} \mathrm{n}^{\prime}}}{\partial \bar{\rho}_{\mathrm{m}^{\prime}}}
$$




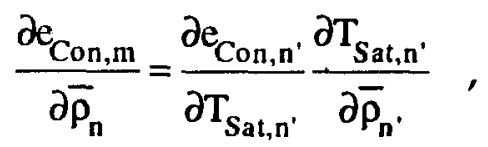

$$
\begin{aligned}
& \frac{\partial \mathrm{e}_{\mathrm{Con}, \mathrm{m}}}{\partial \mathrm{T}_{\mathrm{m}^{\prime}}}=\frac{\partial \mathrm{e}_{\text {Con, } \mathrm{n}^{\prime}}}{\partial \mathrm{T}_{{\text {Sat }, \mathrm{n}^{\prime}}}} \frac{\partial \mathrm{T}_{{\mathrm{Sat}, \mathrm{n}^{\prime}}^{\prime}}}{\partial \mathrm{T}_{\mathrm{m}^{\prime}}} \text {, and } \\
& \frac{\partial e_{\text {Con,m }}}{\partial T_{g}}=\frac{\partial e_{C o n, n^{\prime}}}{\partial T_{\text {Sat, nn }}} \frac{\partial T_{{\text {Sat }, n^{\prime}}^{\prime}}}{\partial T_{g}},
\end{aligned}
$$

with

$$
\begin{aligned}
& \mathrm{m}=\mathrm{m}^{\prime}=\mathrm{L} 1, \mathrm{~L} 2, \text { and } \\
& \mathrm{n}=\mathrm{g} 1, \mathrm{~g} 2 .
\end{aligned}
$$

If $\mathrm{m}=\mathrm{L} 1$, then $\mathrm{n}^{\prime}=\mathrm{g} 1$;

if $\mathrm{m}=\mathrm{L} 2$, then $\mathrm{n}^{\prime}=\mathrm{g} 2$, and

eCon, $m=$ the condensated liquid internal energy for the vapor component $n$ '.

\section{Heat of Vaporization Derivatives}

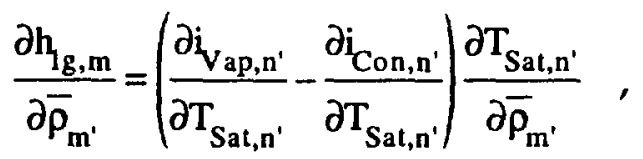

$$
\begin{aligned}
& \frac{\partial \mathrm{h}_{\mathrm{g}, \mathrm{m}}}{\partial \bar{\rho}_{\mathrm{n}}}=\left(\frac{\partial \dot{\mathrm{V}}_{\mathrm{ap}, \mathrm{n}^{\prime}}}{\partial \mathrm{T}_{{\text {Sat }, \mathrm{n}^{\prime}}}}-\frac{\partial \mathrm{i}_{\text {Con, }} \mathrm{n}^{\prime}}{\partial \mathrm{T}_{\text {Sat }, \mathrm{n}^{\prime}}}\right) \frac{\partial \mathrm{T}_{\text {Sat, } \mathbf{n}^{\prime}}}{\partial \bar{\rho}_{\mathrm{n}}}, \\
& \frac{\partial \mathrm{h}_{\mathrm{lg}, \mathrm{m}}}{\partial \mathrm{T}_{\mathrm{m}^{\prime}}}=\left(\frac{\partial \mathrm{i}_{\text {Vap }, \mathrm{n}^{\prime}}}{\partial \mathrm{T}_{{\text {Sat }, \mathrm{n}^{\prime}}}}-\frac{\partial \mathrm{i}_{\text {Con, } \mathrm{n}^{\prime}}}{\partial \mathrm{T}_{{\text {Sat }, \mathrm{n}^{\prime}}}}\right) \frac{\partial \mathrm{T}_{{\text {Sat }, \mathrm{n}^{\prime}}^{\prime}}}{\partial \mathrm{T}_{\mathrm{m}^{\prime}}} \text {, and } \\
& \frac{\partial \mathrm{h}_{\mathrm{g}, \mathrm{m}}}{\partial \mathrm{T}_{\mathrm{g}}}=\left(\frac{\partial \mathrm{i}_{\mathrm{Vap}, \mathrm{n}^{\prime}}}{\partial \mathrm{T}_{{\text {Sat }, \mathrm{n}^{\prime}}}}-\frac{\partial \mathrm{i}_{\mathrm{Con}, \mathrm{n}^{\prime}}}{\left.\partial \mathrm{T}_{{\text {Sat }, \mathrm{n}^{\prime}}}\right)}\right) \frac{\partial \mathrm{T}_{{\mathrm{Sat}, \mathrm{n}^{\prime}}^{\prime}}}{\partial \mathrm{T}_{\mathrm{g}}},
\end{aligned}
$$


with

$$
\begin{aligned}
& \mathrm{m}=\mathrm{m}^{\prime}=\mathrm{L} 1, \mathrm{~L} 2, \text { and } \\
& \mathrm{n}=\mathrm{g} 1, \mathrm{~g} 2 .
\end{aligned}
$$

If $\mathrm{m}=\mathrm{L} 1$, then $\mathrm{n}^{\prime}=\mathrm{g} 1$;

if $m=L 2$, then $n^{\prime}=g 2$.

The term $h_{\mathrm{lg}, \mathrm{m}}$ is the heat of vaporization of the liquid component $m, i_{\text {Con, }}{ }^{\prime}$ is the saturated liquid enthalpy of the vapor component $n^{\prime}$, and ivap, $n^{\prime}$ is the saturated vapor enthalpy of the vapor component n'.

\section{Saturated Vapor Internal Energies}

$$
\begin{aligned}
& \frac{\partial \epsilon_{V_{a p, m}}}{\partial \bar{\rho}_{n^{\prime}}}=\frac{\partial e_{V_{a p, n^{\prime}}}}{\partial T_{S a t, n^{\prime}}} \frac{\partial T_{S_{a t, n^{\prime}}}}{\partial \bar{\rho}_{n^{\prime}}} \text {, and } \\
& \frac{\partial e_{\text {Vap,m }}}{\partial T_{\mathfrak{g}}}=\frac{\partial e_{V_{a p, n^{\prime}}}}{\partial T_{S_{a t, n^{\prime}}}} \frac{\partial T_{S_{a t, n^{\prime}}}}{\partial T_{\mathcal{B}}},
\end{aligned}
$$

with

$$
\begin{aligned}
& \mathrm{m}=\mathrm{L} 1, \mathrm{~L} 2 ; \\
& \mathrm{n}^{\prime}=\mathrm{g} 1, \text { if } \mathrm{m}=\mathrm{L} 1 ; \text { and } \\
& \mathrm{n}^{\prime}=\mathrm{g} 2, \text { if } \mathrm{m}=\mathrm{L} 2 .
\end{aligned}
$$

The term eVap, $n^{\prime}$ is the saturated vapor internal energy for the vapor component $n^{\prime}$.

f. Real Gas Volume Fraction Derivatives. In each numerical cell, the real gas volume fraction is defined as

$$
\alpha_{g}=\left(1-\alpha_{s}\right)-\left(\alpha_{P}+\alpha_{L 1}+\alpha_{L 2}\right)
$$

so the real gas volume fraction derivatives can be calculated with the same expressions used for the effective gas volume fraction in Table III.2, with $\alpha_{0}=0$ and $\alpha_{\text {Geff }}=\alpha \mathrm{g}$.

g. Enthalpy Derivatives. With

$$
i_{m}=e_{m}+p_{m} / \rho_{m}
$$


where

$$
\begin{aligned}
& \mathrm{m}=5,6, \text { we find } \\
& \frac{\partial \mathrm{i}_{\mathrm{m}}}{\partial \mathrm{x}}=\frac{\partial \mathrm{e}_{\mathrm{m}}}{\partial \mathrm{x}}+\frac{1}{\rho_{\mathrm{m}}} \frac{\partial \mathrm{p}_{\mathrm{m}}}{\partial \mathrm{x}}-\frac{\mathrm{p}_{\mathrm{m}}}{\rho_{\mathrm{m}}^{2}} \frac{\partial \rho_{\mathrm{m}}}{\partial \mathrm{x}}
\end{aligned}
$$

where

$$
x=\bar{\rho}_{\mathrm{r}} \text { or } \bar{\rho}_{\mathrm{m}}, \mathrm{T}_{\mathrm{r}}, \mathrm{T}_{\mathrm{g}} .
$$

With

$$
\begin{aligned}
& e_{m}(X)=e_{m}\left[\rho_{m}(x), T_{m}(x)\right] ; \\
& p_{m}(x)=p_{m}\left[\rho_{m}(x), T_{m}(x)\right] ; \text { and } \\
& \rho_{m}(x)=\rho_{m}(x), \text { we find the general equation } \\
& \frac{\partial i_{m}}{\partial x}=\frac{\partial e_{m}}{\partial \rho_{m}} \frac{\partial \rho_{m}}{\partial x}+\frac{\partial e_{m}}{\partial T_{m}} \frac{\partial T_{m}}{\partial x}+\frac{1}{\rho_{m}}\left(\frac{\partial p_{m}}{\partial \rho_{m}} \frac{\partial \rho_{m}}{\partial x}+\frac{\partial \rho_{m}}{\partial T_{m}} \frac{\partial T_{m}}{\partial x}\right)-\frac{p_{m}}{\rho_{m}^{2}} \frac{\partial \rho_{m}}{\partial x}
\end{aligned}
$$

and thus, for

$$
\begin{aligned}
& x=\bar{\rho}_{\mathrm{r}}(\mathrm{r}=3,4): \frac{\partial \mathrm{T}_{\mathrm{m}}}{\partial \bar{\rho}_{\mathrm{r}}}=0, \\
& \frac{\partial \mathrm{i}_{\mathrm{m}}}{\partial \bar{\rho}_{\mathrm{r}}}=\frac{\partial \mathrm{e}_{\mathrm{m}}}{\partial \rho_{\mathrm{m}}} \frac{\partial \rho_{\mathrm{m}}}{\partial \bar{\rho}_{\mathrm{r}}}\left(1+\frac{1}{\rho_{\mathrm{m}}} \frac{\partial \mathrm{p}_{\mathrm{m}}}{\partial \rho_{\mathrm{m}}} / \frac{\partial \mathrm{e}_{\mathrm{m}}}{\partial \rho_{\mathrm{m}}}-\frac{\mathrm{p}_{\mathrm{m}}}{\rho_{\mathrm{m}}^{2}} / \frac{\partial \mathrm{e}_{\mathrm{m}}}{\partial \rho_{\mathrm{m}}}\right) \text {, or with } \\
& \frac{\partial \mathrm{e}_{\mathrm{m}}}{\partial \bar{\rho}_{\mathrm{m}}}=\frac{\partial \mathrm{e}_{\mathrm{m}}}{\partial \rho_{\mathrm{m}}} \frac{\partial \rho_{\mathrm{m}}}{\partial \bar{\rho}_{\mathrm{r}}}+\frac{\partial \mathrm{e}_{\mathrm{m}} \frac{\partial \mathrm{T}_{\mathrm{m}}}{\partial \bar{\rho}_{\mathrm{m}}}}{=0}, \\
& \frac{\partial \mathrm{i}_{\mathrm{m}}}{\partial \bar{\rho}_{\mathrm{r}}}=\frac{\partial \mathrm{e}_{\mathrm{m}}}{\partial \bar{\rho}_{\mathrm{m}}}\left(1+\frac{1}{\rho_{\mathrm{m}}} \frac{\partial \mathrm{p}_{\mathrm{m}}}{\partial \rho_{\mathrm{m}}} / \frac{\partial \mathrm{e}_{\mathrm{m}}}{\partial \rho_{\mathrm{m}}}-\frac{\mathrm{p}_{\mathrm{m}}}{\rho_{\mathrm{m}}^{2}} / \frac{\partial \mathrm{e}_{\mathrm{m}}}{\partial \rho_{\mathrm{m}}}\right) \text { with } \mathrm{m}=5,6 .
\end{aligned}
$$


For $x=\rho_{m}(m=5,6)$, we find accordingly with $\frac{\partial T_{m}}{\partial \bar{\rho}_{m}}=0$,

$$
\frac{\partial i_{m}}{\partial \bar{\rho}_{m}}=\frac{\partial e_{m}}{\partial \bar{\rho}_{m}}\left(1+\frac{1}{\rho_{m}} \frac{\partial p_{m}}{\partial \rho_{m}} / \frac{\partial e_{m}}{\partial \rho_{m}}-\frac{p_{m}}{\rho_{m}^{2}} / \frac{\partial e_{m}}{\partial \rho_{m}}\right)
$$

If $x=T_{r}(r=3,4): \frac{\partial T_{m}}{\partial T_{r}}=0$, then

$$
\frac{\partial \mathrm{i}_{\mathrm{m}}}{\partial \mathrm{T}_{\mathrm{r}}}=\frac{\partial \mathrm{e}_{\mathrm{m}}}{\partial \mathrm{T}_{\mathrm{r}}}\left(1+\frac{1}{\rho_{\mathrm{m}}} \frac{\partial \mathrm{p}_{\mathrm{m}}}{\partial \rho_{\mathrm{m}}} / \frac{\partial \mathrm{e}_{\mathrm{m}}}{\partial \rho_{\mathrm{m}}}-\frac{\mathrm{p}_{\mathrm{m}}}{\rho_{\mathrm{m}}^{2}} / \frac{\partial \mathrm{e}_{\mathrm{m}}}{\partial \rho_{\mathrm{m}}}\right)
$$

With $\mathrm{x}=\mathrm{T}_{\mathrm{G}}$ :

$$
\begin{aligned}
\mathrm{T}_{\mathrm{m}} & =\mathrm{T}_{\mathrm{G}} \text {, and } \\
\partial \mathrm{T}_{\mathrm{m}} / \partial \mathrm{T}_{\mathrm{G}} & =1
\end{aligned}
$$

where

$$
\begin{aligned}
& \mathrm{m}=5,6, \text { general Eq. (3.88) reads } \\
& \frac{\partial \mathrm{i}_{\mathrm{m}}}{\partial \mathrm{T}_{\mathrm{G}}}=\frac{\partial \mathrm{e}_{\mathrm{m}}}{\partial \rho_{\mathrm{m}}} \frac{\partial \rho_{\mathrm{m}}}{\partial \mathrm{T}_{\mathrm{G}}}+\frac{\partial \mathrm{e}_{\mathrm{m}}}{\partial \mathrm{T}_{\mathrm{G}}} \frac{1}{\rho_{\mathrm{m}}}\left(\frac{\partial \mathrm{p}_{\mathrm{m}}}{\partial \rho_{\mathrm{m}}} \frac{\partial \rho_{\mathrm{m}}}{\partial \mathrm{T}_{\mathrm{G}}}+\frac{\partial \mathrm{p}_{\mathrm{m}}}{\partial \mathrm{T}_{\mathrm{G}}}\right)-\frac{\mathrm{p}_{\mathrm{m}}}{\rho_{\mathrm{m}}^{2}} \frac{\partial \rho_{\mathrm{m}}}{\partial \mathrm{T}_{\mathrm{G}}} \text {, or } \\
& \frac{\partial \mathrm{i}_{\mathrm{m}}}{\partial \mathrm{T}_{\mathrm{G}}}=\frac{\partial \mathrm{e}_{\mathrm{m}}}{\partial \mathrm{T}_{\mathrm{G}}} \frac{1}{\rho_{\mathrm{m}}} \frac{\partial \rho_{\mathrm{m}}}{\partial \mathrm{T}_{\mathrm{G}}}+\frac{\partial \mathrm{p}_{\mathrm{m}}}{\partial \mathrm{T}_{\mathrm{G}}}\left(\frac{\partial \mathrm{e}_{\mathrm{m}}}{\partial \rho_{\mathrm{m}}}+\frac{1}{\rho_{\mathrm{m}}} \frac{\partial \mathrm{p}_{\mathrm{m}}}{\partial \rho_{\mathrm{m}}}-\frac{\mathrm{p}_{\mathrm{m}}}{\rho_{\mathrm{m}}^{2}}\right)
\end{aligned}
$$

We have $\rho_{m}\left(T_{G}\right)=\bar{\rho}_{m} / \alpha_{G e f f}\left(T_{G}\right)$; thus,

$$
\begin{aligned}
& \frac{\partial \rho_{m}}{\partial T_{G}}=-\frac{\bar{p}_{m}}{\alpha_{G e f f}^{2}} \frac{\partial \alpha_{G e f f}}{\partial T_{G}} \text {, which leads to } \\
& \frac{\partial \mathrm{i}_{\mathrm{m}}}{\partial \mathrm{T}_{\mathrm{G}}}=\frac{\partial \mathrm{e}_{\mathrm{m}}}{\partial \mathrm{T}_{\mathrm{G}}}+\frac{1}{\rho_{\mathrm{m}}} \frac{\partial \rho_{\mathrm{m}}}{\partial \mathrm{T}_{\mathrm{G}}}-\frac{\overline{\mathrm{p}}_{\mathrm{m}}}{\alpha_{\text {Geff }}^{2}} \frac{\partial \alpha_{\mathrm{Geff}}}{\partial \mathrm{T}_{\mathrm{G}}}\left(\frac{\partial \mathrm{e}_{\mathrm{m}}}{\partial \rho_{\mathrm{m}}}+\frac{1}{\rho_{\mathrm{m}}} \frac{\partial \mathrm{p}_{\mathrm{m}}}{\partial \rho_{\mathrm{m}}}-\frac{\mathrm{p}_{\mathrm{m}}}{\rho_{\mathrm{m}}^{2}}\right)
\end{aligned}
$$




\section{The TEOS Interface Routines}

1. Introduction. In this chapter, the interface routines for the TEOS model are described, i.e., the routines that are called in the initializing and transient phases of the code to supply thermodynamic properties and/or their derivatives, mostly under the assumption of mechanical equilibrium and based on the TEOS.

The flowchart in Fig. 3.11 shows where in the overall procedure the interface routines are called. The flowchart contains the initialization phase and the transient phase consisting of a loop over the four steps of the AFDM algorithm (AFDMS1 through AFDMS4 are the driver routines of the steps). Each timestep in the fluid dynamics solution algorithm requires a cycle around this loop. The branches in steps 1 and 2 correspond to different code options; MASTTP and MASTSP are the mass-transfer driver routines for two-phase and single-phase cells, respectively.

The routines described in this chapter are inserted in the compilable code only if the SESAME option has been set "on" at the time of preprocessing the source code. However, a corresponding set of routines exists for the SESAME option "off," i.e., for the simplified analytical EOS (see Chap. II). For the specification of the routines, see App. B. All routines are vectorized to sweep over the cells specified by the calling parameters of the routines.

2. Initialization Routines. The EOSUP routine is called during the initialization phase of the code by the INIPAR routine. EOSUP calls the routines to load the SESAME tables into the storage arrays and to assign from these those EOS quantities that remain unmodified during the transient phase.

The EOSAT routine is called during the initialization phase of the code by the INICEL routine. EOSAT calculates the properties in the cells from the input quantities under the assumption of thermodynamic equilibrium (see Table IV).

3. Mechanical Equilibrium Routine EOSPEQ and Related Routines. The EOSPEQ routine is called indirectly by all other TEOS interface routines (except EOST) that are used in the transient phase of the code (see Fig. 3.11). EOSPEQ calculates the $\mathrm{p}$ ' sure, volume fractions, and other properties in the cells from given macrodensities and temperatures under the assumption of mechanical equilibrium (see Table V). 


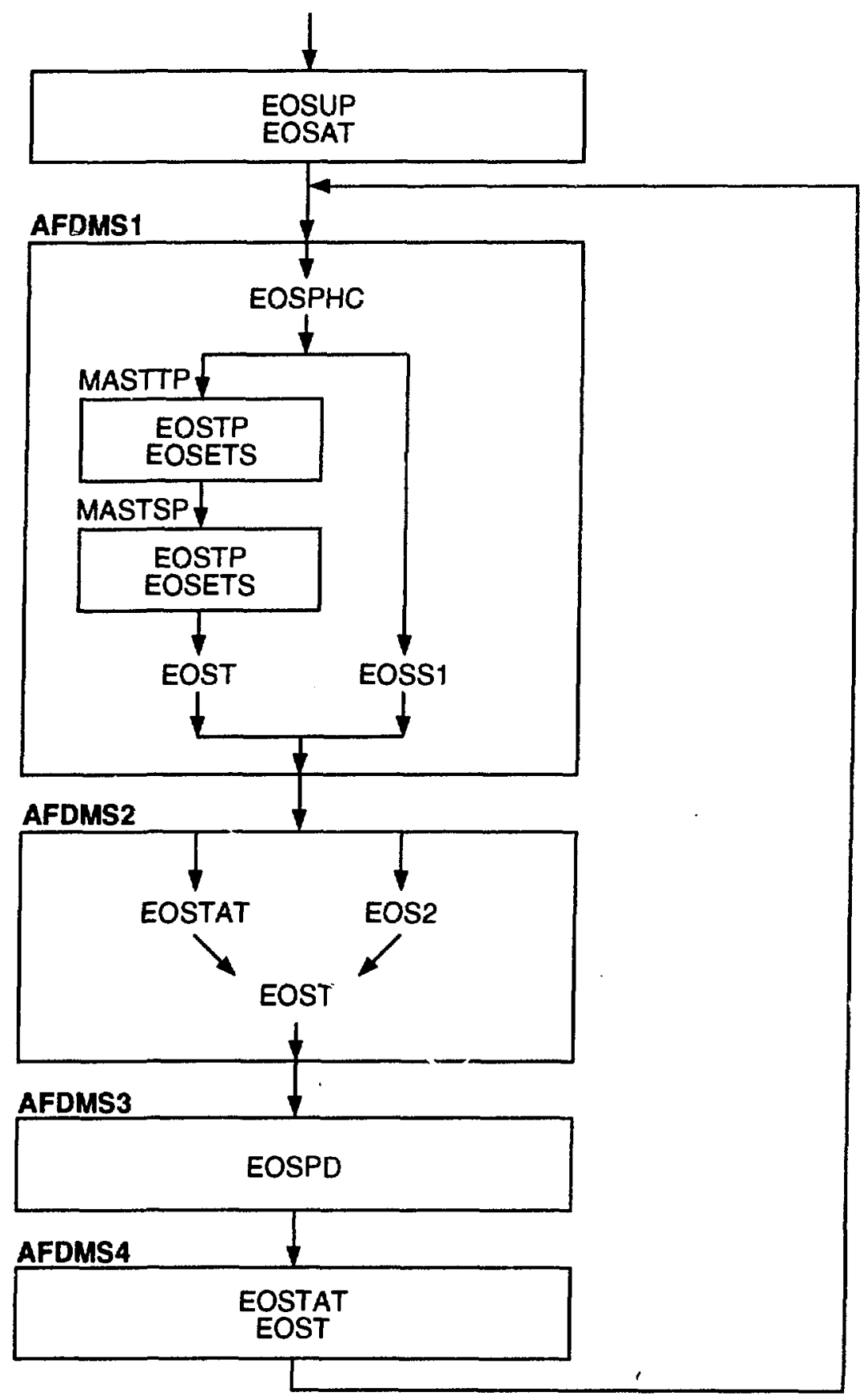

Fig. 3.11.

Calls of the EOS interface routines. 


\section{TABLE IV}

\section{ALGORITIM FOR THE INITIAL THERMODYNAMIC EQUILIBRIUM}

(Nominal case only; no missing components. Note that thermodynamic equilibrium is obtained only if all input temperatures are equal.) For all cells, the vapor mixture volume fraction $\alpha_{5}^{i}=1-\sum_{r=1}^{4} \alpha_{r}^{i}$ is determined from the input volume fractions $\alpha_{\mathrm{r}}^{\mathrm{i}}, \mathrm{r}=1, \ldots, 4$.

For two-phase equilibrium $\left(\alpha_{5}^{i} \geq 0\right)$, the saturation pressures of the two liquid components are calculated at the input temperature $T_{r}^{i}, r=3,4$, and therefore the cell pressure $p$ as the total vapor pressure:

$$
\begin{aligned}
& p_{5}=p_{\text {Sat }, 3}\left(T_{3}^{i}\right), \\
& p_{6}=p_{\text {Sat }, 2}\left(T_{4}^{i}\right), \\
& p_{7}=p_{7}^{i}, \text { and } \\
& p_{7}=p_{5}+p_{6}+p_{7}
\end{aligned}
$$

For single-phase equilibrium $\left(\alpha_{5}^{\mathrm{i}}<0\right)$, the saturation pressures of the two liquid components are calculated at the input temperatures $T_{r}^{j}, r=3,4$. The cell pressure $p$, however, is the input cell pressure:

$$
\begin{aligned}
& \mathrm{p}_{5}=\mathrm{p}_{\text {Sat }, 3}\left(\mathrm{~T}_{3}^{\mathrm{i}}-\Delta \mathrm{T}_{\text {Sup }, 1}\right), \\
& \mathrm{p}_{6}=\mathrm{p}_{\text {Sat }, 2}\left(\mathrm{~T}_{4}^{\mathrm{i}}-\Delta \mathrm{T}_{\text {Sup }, 2}\right), \\
& \mathrm{p}_{7}=\mathrm{p}_{7}^{\mathrm{i}}, \text { and } \\
& \mathrm{p}=\mathrm{p}_{\text {cell }}^{\mathrm{i}} \text {. }
\end{aligned}
$$

Next, for both cases, the component densities $\rho_{m}, m=2, \ldots, 7$, are calculated from the above pressures and from the input temperatures $T_{r}^{i}, r=2, \ldots, 5$, by the requirements

\footnotetext{
* The default values of the material index $M^{\prime}$ (see App. A) are used for the TEOS functions.
} 


\section{TABLE IV (CONT.)}

$$
\begin{gathered}
p_{\text {Sld, }, 5}\left(\rho_{2}, T_{2}^{i}\right)=p, \\
p_{V_{a n, M^{\prime}(m)}}\left(\rho_{m}, T_{r(m)}^{i}\right)=p \quad \text { for } m=3,4 \text {, and } \\
p_{V_{a n, M^{\prime}(m)}}\left(\rho_{m}, T_{r(m)}^{i}\right) 5=p_{m} \quad \text { for } m=5,6,7,
\end{gathered}
$$

These equations of the general form $p_{m}\left(\rho_{m}, T_{r}^{i}\right)=p_{0}$ are solved by a Newton algorithm iterating

$$
\rho_{\mathrm{m}} \leftarrow \rho_{\mathrm{m}}-\frac{\mathrm{p}_{\mathrm{m}}-\mathrm{p}_{0}}{\partial_{\mathrm{\rho}} \mathrm{p}_{\mathrm{m}}}
$$

starting with $\rho_{m}=\rho$ Sus, $5, \rho_{C o n}, M^{\prime}(m)$, or $\rho_{V a p, M^{\prime}(m)}$, respectively.

With the component densities known, the component specific internal energies $e_{\mathbf{r}}$ $\mathrm{r}=2,3,4, \mathrm{G}$, are calculated straightforwardly:

$$
\begin{gathered}
e_{2}=e_{S I d, 5}\left(\rho_{2}, T_{2}^{i}\right), \\
e_{r}=e_{V a n, M^{\prime}(r)}\left(\rho_{m(r)}, T_{r}^{i}\right) \quad \text { for } r=3,4 \text {, and } \\
e_{G}=\sum_{m=}^{5 \ldots 7} \rho_{m} e_{\operatorname{Van}, M^{\prime}(r)}\left(\rho_{m}, T_{5}^{i}\right) / \sum_{m=}^{5 \ldots 7} \rho_{m}
\end{gathered}
$$

The structure component density $\rho_{1}$ and specific internal energy $e_{1}$ are taken as

$$
\rho_{1}=\rho_{\text {Sus }, 4}\left(T_{1}^{i}\right) \quad e_{1}=e_{\text {Sus }, 4}\left(T_{1}^{i}\right)
$$

If the input volume fractions result in overfilled single-phase cells, they are normalized with the factor $\mathrm{R}$ : 


\section{TABLE IV (CONT.)}

$$
\text { If } \alpha_{5}^{i}<0: R=\frac{1-\alpha_{1}^{i}}{\alpha_{2}^{i}+\alpha_{3}^{i}+\alpha_{4}^{i}} ; \text { else } R=1 \text {, }
$$

resulting in the component macrodensities and output volume fractions:

$$
\begin{gathered}
\bar{\rho}_{1}=\alpha_{1}^{i} \rho_{1}, \\
\bar{\rho}_{m}=R \alpha_{r(m)}^{i} \rho_{m} \quad \text { for } m=2,3,4, \\
\bar{\rho}_{m}=\alpha_{g e} \rho_{m} \quad \text { for } m=5,6,7, \text { and } \\
\alpha_{m}=\bar{\rho}_{m} / \rho_{m} \quad \text { for } m=1, \ldots, 4
\end{gathered}
$$

If $\alpha_{5}^{i}>0: \alpha_{5}=1-\alpha_{1}-\alpha_{2}-\alpha_{3}-\alpha_{4} ;$ else: $\alpha_{5}=-\varepsilon$,

with

$$
\alpha_{g e}=\max \left[\alpha_{5}+\alpha_{0}\left(\alpha_{2}+\alpha_{3}+\alpha_{4}\right), \alpha_{0}\left(1-\alpha_{1}\right)\right]
$$

\section{TABLE V}

\section{ALGORITHM FOR THE MECHANICAL EQUILIBRIUM}

(Nominal case only, no missing components, no spinodal limits, etc.) Starting from given macrodensities $\bar{\rho}_{m}, m=1, \ldots, 7$, and temperatures ${ }^{*} T_{m}, m=1, \ldots, 5$, for all cells, the mechanical equilibrium is calculated assuming a two-phase state. Then, for those cells that from the first calculation turn out to be in single-phase state, the mechanical equilibrium is recalculated assuming single-phase state.

\footnotetext{
*For temperatures, the subscript $m$ is used here instead of $r$ because both are identical for $m=2,3,4$.
} 


\section{TABLE V (CONT.)}

Two-phase mechanical equilibrium: The requirement is

$$
\delta p_{m} \equiv p_{m}\left(\rho_{m}, T_{m}\right)-\sum_{n=}^{5} p_{n}^{7}\left(\rho_{n}, T_{5}\right)=0 \quad \text { for } m=2,3,4
$$

where

$$
\begin{gathered}
\rho_{\mathrm{m}}=\frac{\bar{\rho}_{\mathrm{m}}}{\alpha_{\mathrm{m}}} \quad \text { for } \mathrm{m}=2,3,4, \\
\rho_{\mathrm{n}}=\frac{\bar{\rho}_{\mathrm{n}}}{\alpha_{\mathrm{ge}}} \quad \text { for } \mathrm{n}=5,6,7 \text {, and } \\
\alpha_{\mathrm{ge}}=\max \left[\alpha_{\mathrm{Geff}}, \alpha_{0}\left(1-\alpha_{1}\right)\right] \quad \alpha_{\mathrm{Geff}}=1-\alpha_{1}-\left(1-\alpha_{0}\right)\left(\alpha_{2}+\alpha_{3}+\alpha_{4}\right) .
\end{gathered}
$$

Thus,

$$
\delta p_{m}\left(\alpha_{2}, \alpha_{3}, \alpha_{4}\right) \equiv p_{m}\left(\frac{\bar{\rho}_{m}}{\alpha_{m}}, T_{m}\right)-\sum_{n=}^{5} p_{n}^{7}\left(\frac{\bar{\rho}_{n}}{\alpha_{g e}\left(\alpha_{2}, \alpha_{3}, \alpha_{4}\right)}, T_{5}\right)=0
$$

$$
\text { for } m=2,3,4
$$

or in matrix notation:

$$
\delta p(\alpha)=0
$$

where

$$
\delta p \equiv\left(\begin{array}{c}
\delta p_{2} \\
\delta p_{3} \\
\delta p_{4}
\end{array}\right) \quad \underline{\alpha} \equiv\left(\begin{array}{c}
\alpha_{2} \\
\alpha_{3} \\
\alpha_{4}
\end{array}\right)
$$

This nonlinear matrix equation is solved by a generalized Newton algorithm iterating

$$
\underline{\alpha} \leftarrow \underline{\alpha}-\left(\frac{\mathrm{d} \delta \mathbf{p}}{\mathrm{d} \alpha^{\mathrm{T}}}\right)^{-1} \delta \mathbf{p}
$$




\section{TABLE V (CONT.)}

where

$$
\frac{\mathrm{d} \delta \mathbf{p}}{\mathrm{d} \alpha^{\mathrm{T}}} \equiv\left(\begin{array}{ccc}
\frac{\partial \delta \mathrm{p}_{2}}{\partial \alpha_{2}} & \cdots & \frac{\partial \delta \mathrm{p}_{2}}{\partial \alpha_{4}} \\
\vdots & & \vdots \\
\frac{\partial \delta \mathrm{p}_{4}}{\partial \alpha_{2}} & \cdots & \frac{\partial \delta p_{4}}{\partial \alpha_{4}}
\end{array}\right) .
$$

The derivatives in the above matrix elements are calculated using the basic derivatives of $p_{m}$ with respect to $\rho_{m}$ :

$$
\begin{aligned}
\frac{\partial \delta p_{m}}{\partial \alpha_{m^{\prime}}} & =\frac{\partial p_{m}}{\partial \alpha_{m^{\prime}}}-\sum_{n=}^{5 \ldots 7} \frac{\partial p_{n}}{\partial \alpha_{g e}} \frac{\partial \alpha_{g e}}{\partial \alpha_{m^{\prime}}} \\
& =\underbrace{\frac{\partial p_{m}}{\partial p_{m^{\prime}}} \frac{\rho_{m^{\prime}}}{\alpha_{m^{\prime}}}}_{\text {if } m=m^{\prime} \text { only }}-\underbrace{\left(1-\alpha_{0}\right) \sum_{n=1}^{5 \ldots 7} \frac{\partial p_{n}}{\partial \rho_{n}} \frac{\rho_{n}}{\alpha_{g e}}}_{\text {if } \alpha_{g e}>\alpha_{0}\left(1-\alpha_{1}\right) \text { only }} \text { for } m=2,3,4, m^{\prime}=2,3,4 .
\end{aligned}
$$

Single-phase mechanical equilibrium: if $\alpha_{\text {Geff }}<\alpha_{0}\left(1-\alpha_{1}\right)$, the requirement is

$$
\delta p_{m} \equiv p_{m}\left(\rho_{m}, T_{m}\right)-p_{4}\left(\rho_{4}, T_{4}\right)=0 \text { for } m=2,3
$$

where

$$
\begin{gathered}
\rho_{\mathrm{m}}=\frac{\bar{\rho}_{\mathrm{m}}}{\alpha_{\mathrm{m}}} \text { for } \mathrm{m}=2,3,4, \text { and } \\
\alpha_{4}=1-\alpha_{1}-\left(\alpha_{2}+\alpha_{3}\right) .
\end{gathered}
$$


Thus,

$$
\delta p_{m}\left(\alpha_{2}, \alpha_{3}\right) \equiv p_{m}\left(\frac{\bar{\rho}_{m}}{\alpha_{m}}, T_{m}\right)-p_{4}\left(\frac{\bar{\rho}_{4}}{\alpha_{4}\left(\alpha_{2}, \alpha_{3}\right)}, T_{4}\right)=0 \quad \text { for } m=2,3
$$

or in matrix notation,

$$
\delta p(\underline{\alpha})=0
$$

where

$$
\delta p \equiv\left(\begin{array}{c}
\delta \mathrm{p}_{2} \\
\delta \mathrm{p}_{3}
\end{array}\right) \quad \underline{\alpha} \equiv\left(\begin{array}{l}
\alpha_{2} \\
\alpha_{3}
\end{array}\right)
$$

This nonlinear matrix equation is solved by a generalized Newton algorithm iterating

$$
\underline{\alpha} \leftarrow \underline{\alpha}-\left(\frac{\mathrm{d} \delta p}{d \alpha^{T}}\right)^{-1} \delta p
$$

where

$$
\frac{\mathrm{d} \delta \mathrm{p}}{\mathrm{d} \alpha^{\mathrm{T}}} \equiv\left(\begin{array}{ll}
\frac{\partial \delta \mathrm{p}_{2}}{\partial \alpha_{2}} & \frac{\partial \delta \mathrm{p}_{2}}{\partial \alpha_{3}} \\
\frac{\partial \delta \mathrm{p}_{3}}{\partial \alpha_{2}} & \frac{\partial \delta \mathrm{p}_{3}}{\partial \alpha_{3}}
\end{array}\right)
$$

and

$$
\frac{\partial \delta p_{m}}{\partial \alpha_{m^{\prime}}}=\frac{\partial p_{m}}{\partial \alpha_{m^{\prime}}}-\frac{\partial p_{4}}{\partial \alpha_{4}} \frac{\partial \alpha_{4}}{\partial \alpha_{m^{\prime}}}=-\underbrace{\frac{\partial p_{m}}{\partial \rho_{m^{\prime}}} \frac{\rho_{m^{\prime}}}{\alpha_{m^{\prime}}}}_{\text {if } m=m^{\prime} \text { only }}-\frac{\partial p_{4}}{\partial \rho_{4}} \frac{\rho_{4}}{\alpha_{m^{\prime}}} \quad \text { for } m=2,3, \quad m^{\prime}=2,3
$$


The EOS2, EOSTAT, and EOSETS routines are more or less redundant routines that branch to EOSPEQ and virtually return a subset of the quantities calculated by the routine. The EOS2 routine returns the pressure, component volume fractions, densities, and specific internal energies of the cells. The EOSTAT routine does the same; however, it returns the vapor mixture specific internal energy instead of the individual vapor component energies and, in addition, calculates the saturation temperatures. The EOSETS routines are needed to return the component and vapor mixture specific internal energies only.

EOSPEQ has no correspondence in the SESAME option "off."

4. Temperature Inversion Routine EOST and Related Routines. The EOST routine is called directly in steps 1,2 , and 4 of the code. EOST calculates the temperatures and other properties in the cells from given macrodensities and specific internal energies under the assumption of mechanical equilibrium (see Table VI).

5. Routines Calculating Derivatives. The six remaining interface routines not dealt with in the previous sections supply various thermodynamic properties and their derivatives under the assumption of mechanical equilibrium. (Note that derivation under the assumption of mechanical equilibrium means a total derivation with respect to $\bar{\rho}_{\mathrm{m}}$ or $T_{\mathrm{r}}$ as compared to the partial derivatives with respect to $\alpha_{\mathrm{r}}$ and $T_{r}$ used in Tables V and VI; see Chap. III.C.) Figure 3.12 maps the cross reference between the set of occurring derivatives and interface routines. Many of the derivatives are calculated in more than one routine, i.e., the same algorithms occur repeatedly in several routines, and the coding for identical algorithms is not always identical. There are historical reasons for this redundancy (see Acknowledgments). Specialization of the TEOS interface routines to the intended applications is incomplete, and further effort in this area appears desirable.

The EOSPHC routine is called in step 1 of the code to process all cells. The EOSTP routine is called in step 1 to process two-phase cells only. The EOSD2 and EOSVD1 routines are called in step 1 to process single-phase cells only. The EOSS1 routine is called in step 1 instead of the previous three routines if the NOPHASE option is "on." Finally, the EOSPD routine is called in step 3. All these routines call the EOSPEQ routine.

For the formulae of the derivatives coded in these routines, see Chap. III.C. 


\section{TABLE VI}

\section{ALGORITHM FOR THE TERMPERATURE INVERSION}

(Nominal case only, no missing components, no spinodal limits, etc.) Starting from given macrodensities $\bar{\rho}_{\mathrm{m}}, \mathrm{m}=1, \ldots, 7$, and specific internal energies* $\overline{\mathrm{e}}_{\mathrm{m}}, \mathrm{m}=1, \ldots, 4, \mathrm{G}$, for all cells the mechanical equilibrium and the temperatures are calculated simultaneously, assuming a two-phase state. Then for those cells that from the first calculation turn out to be single-phase state, the mechanical equilibrium and the temperatures are recalculated, assuming single-phase state.

Two-phase mechanical equilibrium: The requirement is

$$
\begin{gathered}
\delta p_{m} \equiv p_{m}\left(\rho_{m}, T_{m}\right)-\sum_{n=}^{5} p_{n}^{7}\left(\rho_{n}, T_{5}\right)=0 \quad \text { for } m=2,3,4 \\
\delta e_{m} \equiv e_{m}\left(\rho_{m}, T_{m}\right)-\bar{e}_{m}=0 \quad \text { for } m=2,3,4, \text { and } \\
\delta e_{G} \equiv \sum_{n=}^{5 \ldots 7} x_{n} e_{n}\left(\rho_{n}, T_{5}\right)-\bar{e}_{G}=0
\end{gathered}
$$

where

$$
\begin{gathered}
\rho_{m}=\frac{\bar{\rho}_{m}}{\alpha_{m}} \text { for } m=2,3,4, \\
\rho_{n}=\frac{\bar{\rho}_{n}}{\alpha_{g e}} \text { for } n=5,6,7, \\
\alpha_{g e}=\max \left[\alpha_{G e f f}, \alpha_{0}\left(1-\alpha_{1}\right)\right] \quad \alpha_{\text {Geff }}=1-\alpha_{1}-\left(1-\alpha_{0}\right)\left(\alpha_{2}+\alpha_{3}+\alpha_{4}\right), \text { and } \\
x_{n}=\bar{\rho}_{n} /\left(\bar{\rho}_{5}+\bar{\rho}_{6}+\bar{\rho}_{7}\right) \text { for } n=5,6,7 .
\end{gathered}
$$

Thus,

$$
\delta \mathrm{p}_{\mathrm{m}}\left(\alpha_{2}, \alpha_{3}, \alpha_{4}, \mathrm{~T}_{\mathrm{m}}, \mathrm{T}_{5}\right) \equiv \mathrm{p}_{\mathrm{m}}\left(\frac{\bar{\rho}_{\mathrm{m}}}{\alpha_{\mathrm{m}}}, \mathrm{T}_{\mathrm{m}}\right)-\sum_{\mathrm{n}=\mathrm{m}}^{5} \mathrm{p}_{\mathrm{n}}\left(\frac{\bar{\rho}_{\mathrm{n}}}{\alpha_{\mathrm{ge}}\left(\alpha_{2}, \alpha_{3}, \alpha_{4}\right)}, \mathrm{T}_{5}\right)=0 \quad \text { for } \mathrm{m}=2,3,4,
$$

\footnotetext{
*For energies and temperatures, the subscript $m$ is used here instead of $r$ because both are identical for $\mathrm{m}=\mathbf{2 , 3 , 4}$.
} 


\section{TABLE VI (CONT.)}

$$
\begin{aligned}
& \delta e_{m}\left(\alpha_{m}, T_{m}\right) \equiv e_{m}\left(\frac{\bar{\rho}_{m}}{\alpha_{m}}, T_{m}\right)-\bar{e}_{m}=0 \quad \text { for } m=2,3,4, \text { and } \\
& \delta e_{G}\left(\alpha_{m}, T_{5}\right) \equiv \sum_{n=}^{5} x_{n} e_{n}\left(\frac{\bar{\rho}_{n}}{\alpha_{g e}\left(\alpha_{2}, \alpha_{3}, \alpha_{4}\right)}, T_{5}\right)-\bar{e}_{G}=0,
\end{aligned}
$$

or in matrix notation

$$
\delta f(x)=0
$$

where

$$
\delta \mathrm{f} \equiv\left(\begin{array}{c}
\delta \mathrm{p} \\
\delta \mathrm{e}
\end{array}\right) \equiv\left(\begin{array}{c}
\delta \mathrm{p}_{2} \\
\delta \mathrm{p}_{3} \\
\delta \mathrm{p}_{4} \\
\delta \mathrm{e}_{2} \\
\delta \mathrm{e}_{3} \\
\delta \mathrm{e}_{4} \\
\delta \mathrm{e}_{\mathrm{G}}
\end{array}\right) \quad \mathrm{x} \equiv\left(\frac{\alpha}{\mathrm{T}}\right) \equiv\left(\begin{array}{c}
\alpha_{2} \\
\alpha_{3} \\
\alpha_{4} \\
\mathrm{~T}_{2} \\
\mathrm{~T}_{3} \\
\mathrm{~T}_{4} \\
\mathrm{~T}_{5}
\end{array}\right)
$$

This nonlinear matrix equation is solved by a generalized Newton algorithm iterating

$$
\mathbf{x} \leftarrow \mathbf{x}+\delta \mathbf{x}
$$

where

$$
\delta \mathrm{x} \equiv\left(\begin{array}{l}
\delta \underline{\alpha} \\
\delta \mathrm{T}
\end{array}\right) \equiv\left(\begin{array}{c}
\delta \alpha_{2} \\
\delta \alpha_{3} \\
\delta \alpha_{4} \\
\delta \mathrm{T}_{2} \\
\delta \mathrm{T}_{3} \\
\delta \mathrm{T}_{4} \\
\delta \mathrm{T}_{5}
\end{array}\right)
$$




\section{TABLE VI (CONT.)}

is calculated from the system of linear equations

$$
\left(\frac{d \delta f}{d x}\right) \delta x=-\delta f
$$

The matrix of this system is

$$
\begin{aligned}
& \frac{d \delta \mathbf{f}}{d \mathbf{x}^{T}} \equiv\left(\begin{array}{ll}
\frac{\partial \delta p}{T} & \frac{\partial \delta p}{\partial \underline{T}} \\
\frac{\partial \delta \mathbf{e}}{\partial{ }^{T}} & \frac{\partial \delta \mathbf{e}}{\partial \mathbf{T}^{T}}
\end{array}\right)
\end{aligned}
$$

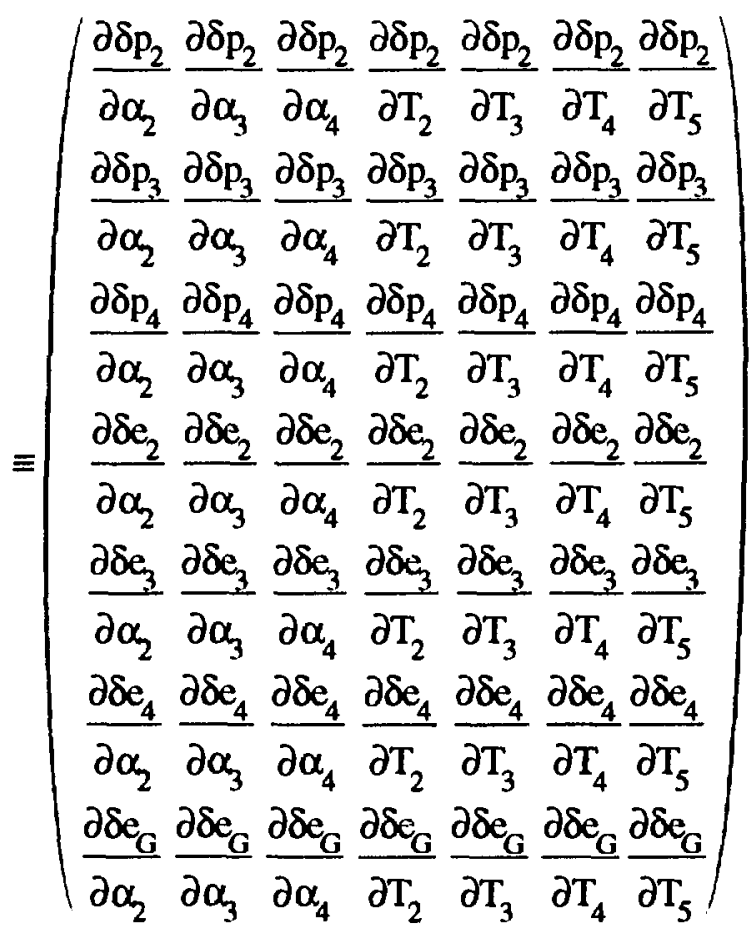

It is seen immediately from Eq. (7) that many elements of the off-diagonal submatrices are zero and that the $\frac{\partial \alpha e}{\partial T}$ submatrix is diagonal and thus can be inverted straightforwardly. This leads to a reduction of the order of the system of linear 


\section{TABLE VI (CONT.)}

equations to be solved simultaneously. In analogy to the Gauß elimination method for a $2 \times 2$ system of linear equations, the block matrix equation

$$
\left(\begin{array}{ll}
\frac{\partial \delta \mathbf{p}}{\mathrm{T}} & \frac{\partial \delta \mathbf{p}}{\mathrm{T}} \\
\frac{\partial \mathbf{T}}{\partial \delta \mathbf{e}} & \frac{\partial \delta \mathbf{e}}{\partial \underline{\underline{T}}}
\end{array}\right)\left(\begin{array}{c}
\delta \underline{\alpha} \\
\delta \mathbf{T}
\end{array}\right)=-\left(\begin{array}{l}
\delta \mathbf{p} \\
\delta \mathbf{e}
\end{array}\right)
$$

is multiplied from the left by the block matrix

$$
\left(\begin{array}{cc}
\mathbf{I} & -\frac{\partial \delta \mathbf{p}}{\partial \mathbf{T}}\left(\frac{\partial \delta \mathbf{e}}{\partial \mathbf{T}}\right)^{-1} \\
\mathbf{0} & \mathbf{I}
\end{array}\right) .
$$

The resulting system can be separated into the system of linear equations

$$
\left(\frac{\partial \delta \mathbf{p}}{\partial \underline{\alpha}}-\frac{\partial \delta p}{\partial \mathbf{T}^{\mathrm{T}}}\left(\frac{\partial \delta \mathbf{e}}{\partial \mathbf{T}}\right)^{-1} \frac{\partial \delta \mathbf{e}}{\partial \underline{\alpha}}\right) \delta \underline{\alpha}=-\delta \mathbf{p}+\frac{\partial \delta \mathbf{p}}{\partial \mathbf{T}}\left(\frac{\partial \delta \mathbf{e}}{\partial \mathbf{T}}\right)^{-1} \delta \mathbf{e},
$$

which must be solved for $\delta \underline{\alpha}$ and into the following system

$$
\frac{\partial \delta \mathbf{e}}{\partial \underline{\underline{T}}} \delta \underline{\alpha}+\left(\frac{\partial \delta \mathbf{e}}{\partial \mathbf{T}}\right) \delta \mathbf{T}=-\delta \dot{e}
$$

which can be straightforwardly solved for $\delta \mathrm{T}$ once $\delta \underline{\alpha}$ is known.

If we denote the first system (5) by

$$
\mathbf{A} \delta \underline{\alpha}=\mathbf{b} \text {, }
$$




\section{TABLE VI (CONT.)}

the elements of the $3 \times 3$ matrix $A$ and of the $3 \times 1$ matrix $b$ are

$$
\begin{aligned}
& \mathrm{a}_{11}=\frac{\partial \delta \mathrm{p}_{2}}{\partial \alpha_{2}}-\frac{\partial \delta \mathrm{p}_{2}}{\partial \mathrm{T}_{2}} \frac{\partial \delta \mathrm{e}_{2}}{\partial \alpha_{2}} / \frac{\partial \delta \mathrm{e}_{2}}{\partial \mathrm{T}_{2}}-\frac{\partial \delta \mathrm{p}_{2}}{\partial \mathrm{T}_{5}} \frac{\partial \mathrm{e}_{\mathrm{G}}}{\partial \alpha_{2}} / \frac{\partial \delta \mathrm{e}_{\mathrm{G}}}{\partial \mathrm{T}_{5}}, \\
& \mathrm{a}_{12}=\frac{\partial \delta \mathrm{p}_{2}}{\partial \alpha_{3}}-\frac{\partial \delta \mathrm{p}_{2}}{\partial \mathrm{T}_{5}} \frac{\partial \delta \mathrm{e}_{\mathrm{G}}}{\partial \alpha_{3}} / \frac{\partial \delta \mathrm{e}_{\mathrm{G}}}{\partial \mathrm{T}_{5}} \text {, } \\
& \mathrm{a}_{13}=\frac{\partial \delta \mathrm{p}_{2}}{\partial \alpha_{4}}-\frac{\partial \delta \mathrm{p}_{2}}{\partial \mathrm{T}_{5}} \frac{\partial \delta \mathrm{e}_{\mathrm{G}}}{\partial \alpha_{4}} / \frac{\partial \delta \mathrm{e}_{\mathrm{G}}}{\partial \mathrm{T}_{5}} \\
& a_{21}=\frac{\partial \delta p_{3}}{\partial \alpha_{2}}-\frac{\partial \delta \mathrm{p}_{3}}{\partial \mathrm{T}_{5}} \frac{\partial \delta \mathrm{e}_{\mathrm{G}}}{\partial \alpha_{2}} / \frac{\partial \delta \mathrm{e}_{\mathrm{G}}}{\partial \mathrm{T}_{5}} \text {, } \\
& a_{22}=\frac{\partial \delta p_{3}}{\partial \alpha_{3}}-\frac{\partial \delta p_{3}}{\partial T_{3}} \frac{\partial \delta e_{3}}{\partial \alpha_{3}} / \frac{\partial \delta e_{3}}{\partial T_{3}}-\frac{\partial \delta p_{3}}{\partial T_{5}} \frac{\partial \delta e_{G}}{\partial \alpha_{3}} / \frac{\partial \delta e_{G}}{\partial T_{5}} \text {, } \\
& \mathrm{a}_{23}=\frac{\partial \delta \mathrm{p}_{3}}{\partial \alpha_{4}}-\frac{\partial \delta \mathrm{p}_{3}}{\partial \mathrm{T}_{5}} \frac{\partial \delta \mathrm{e}_{\mathrm{G}}}{\partial \alpha_{4}} / \frac{\partial \delta \mathrm{e}_{\mathrm{G}}}{\partial \mathrm{T}_{5}} \text {, } \\
& a_{31}=\frac{\partial \delta \mathrm{p}_{4}}{\partial \alpha_{2}}-\frac{\partial \delta \mathrm{p}_{4}}{\partial \mathrm{T}_{5}} \frac{\partial \delta \mathrm{e}_{\mathrm{G}}}{\partial \alpha_{2}} / \frac{\partial \delta \mathrm{e}_{\mathrm{G}}}{\partial \mathrm{T}_{5}} \text {, } \\
& a_{32}=\frac{\partial \delta \mathrm{p}_{4}}{\partial \alpha_{3}}-\frac{\partial \delta \mathrm{p}_{4}}{\partial \mathrm{T}_{5}} \frac{\partial \delta \mathrm{e}_{\mathrm{G}}}{\partial \alpha_{3}} / \frac{\partial \delta \mathrm{e}_{\mathrm{G}}}{\partial \mathrm{T}_{5}} \text {. } \\
& \mathrm{a}_{33}=\frac{\partial \delta \mathrm{p}_{4}}{\partial \alpha_{4}}-\frac{\partial \delta \mathrm{p}_{4}}{\partial \mathrm{T}_{4}} \frac{\partial \delta \mathrm{e}_{4}}{\partial \alpha_{4}} / \frac{\partial \delta \mathrm{e}_{4}}{\partial \mathrm{T}_{4}}-\frac{\partial \delta \mathrm{p}_{4}}{\partial \mathrm{T}_{5}} \frac{\partial \delta \mathrm{e}_{\mathrm{G}}}{\partial \alpha_{4}} / \frac{\partial \delta \mathrm{e}_{\mathrm{G}}}{\partial \mathrm{T}_{5}} \text {, } \\
& \mathrm{b}_{1}=-\delta \mathrm{p}_{2}+\frac{\partial \delta \mathrm{p}_{2}}{\partial \mathrm{T}_{2}} \delta \mathrm{e}_{2} / \frac{\partial \delta \mathrm{e}_{2}}{\partial \mathrm{T}_{2}}+\frac{\partial \delta \mathrm{p}_{2}}{\partial \mathrm{T}_{5}} \delta \mathrm{e}_{\mathrm{G}} / \frac{\partial \delta \mathrm{e}_{\mathrm{G}}}{\partial \mathrm{T}_{5}} \text { ， } \\
& \mathrm{b}_{2}=-\delta \mathrm{p}_{3}+\frac{\partial \delta \mathrm{p}_{3}}{\partial \mathrm{T}_{3}} \delta \mathrm{e}_{3} / \frac{\partial \delta \mathrm{e}_{3}}{\partial \mathrm{T}_{3}}+\frac{\partial \delta \mathrm{p}_{3}}{\partial \mathrm{T}_{5}} \delta \mathrm{e}_{\mathrm{G}} / \frac{\partial \delta \mathrm{e}_{\mathrm{G}}}{\partial \mathrm{T}_{5}} \text {, and } \\
& \mathrm{b}_{3}=-\delta \mathrm{p}_{4}+\frac{\partial \delta \mathrm{p}_{4}}{\partial \mathrm{T}_{4}} \delta \mathrm{e}_{4} / \frac{\partial \delta \mathrm{e}_{4}}{\partial \mathrm{T}_{4}}+\frac{\partial \delta \mathrm{p}_{4}}{\partial \mathrm{T}_{5}} \delta \mathrm{e}_{\mathrm{G}} / \frac{\partial \delta \mathrm{e}_{\mathrm{G}}}{\partial \mathrm{T}_{5}}
\end{aligned}
$$

If we denote the second system (6) by

$$
\delta \mathrm{T}=\mathrm{C} \delta \underline{\alpha}+\mathrm{d},
$$




\section{TABLE VI (CONT.)}

the elements of the $4 \times 3$ matrix $C$ and of the $4 \times 1$ matrix $\mathrm{d}$ are (nonzero elements only)

$$
\begin{aligned}
& c_{11}=-\frac{\partial \delta e_{2}}{\partial \alpha_{2}} / \frac{\partial \delta e_{2}}{\partial T_{2}}, \\
& c_{22}=-\frac{\partial \delta \mathrm{e}_{3}}{\partial \alpha_{3}} / \frac{\partial \delta \mathrm{e}_{3}}{\partial \mathrm{T}_{3}} \text {, } \\
& c_{33}=-\frac{\partial \delta \mathrm{e}_{4}}{\partial \alpha_{4}} / \frac{\partial \delta \mathrm{e}_{4}}{\partial \mathrm{T}_{4}} \text {, } \\
& c_{41}=-\frac{\partial \delta \mathrm{e}_{\mathrm{G}}}{\partial \alpha_{2}} / \frac{\partial \delta \mathrm{e}_{\mathrm{G}}}{\partial \mathrm{T}_{5}}, \\
& c_{42}=-\frac{\partial \delta \mathrm{e}_{\mathrm{G}}}{\partial \alpha_{3}} / \frac{\partial \delta \mathrm{e}_{\mathrm{G}}}{\partial \mathrm{T}_{5}} \text {, } \\
& c_{43}=-\frac{\partial \delta \mathrm{e}_{\mathrm{G}}}{\partial \alpha_{4}} / \frac{\partial \delta \mathrm{e}_{\mathrm{G}}}{\partial \mathrm{T}_{5}} \text {, } \\
& \mathrm{d}_{1}=-\partial \delta \mathrm{e}_{2} / \frac{\partial \delta \mathrm{e}_{2}}{\partial \mathrm{T}_{2}} \text {, } \\
& \mathrm{d}_{2}=-\partial \delta \mathrm{e}_{3} / \frac{\partial \delta \mathrm{e}_{3}}{\partial \mathrm{T}_{3}} \text {, } \\
& d_{3}=-\partial \delta \mathrm{e}_{4} / \frac{\partial \delta \mathrm{e}_{4}}{\partial \mathrm{T}_{4}} \text {, and } \\
& \mathrm{d}_{4}=-\partial \delta \mathrm{e}_{\mathrm{G}} / \frac{\partial \delta \mathrm{e}_{\mathrm{G}}}{\partial \mathrm{T}_{5}} \text {. }
\end{aligned}
$$

The derivatives in the above matrix elements are calculated using the basic derivatives of $p_{m}$ and $e_{m}$ with respect to $\rho_{m}$ and $T_{m}$ :

$$
\frac{\partial \delta \mathrm{p}_{\mathrm{m}}}{\partial \alpha_{\mathrm{m}}}=\underbrace{\frac{\partial \mathrm{p}_{\mathrm{m}}}{\partial \rho_{\mathrm{m}}} \frac{\rho_{\mathrm{m} \mathrm{m}^{\prime}}}{\alpha_{\mathrm{m} \mathrm{m}^{\prime}}}}_{\text {if } \mathrm{m}=\mathrm{m}^{\prime} \text { only }}-\underbrace{\left(1-\alpha_{0}\right) \sum_{\mathrm{n}=\mathrm{I}^{7}} \frac{\partial \mathrm{p}_{\mathrm{n}}}{\partial \rho_{\mathrm{n}}} \frac{\rho_{\mathrm{n}}}{\alpha_{\mathrm{gc}}}}_{\text {if } \alpha_{\mathrm{gc}}>\alpha_{0}\left(1-\alpha_{1}\right) \text { only }} \text { for } \mathrm{m}=2,3,4, \mathrm{~m}^{\prime}=2,3,4,
$$


TABLE VI (CONT.)

$$
\begin{gathered}
\frac{\partial \delta p_{m}}{\partial T_{m}}=\frac{\partial p_{m}}{\partial T_{m}} \frac{\partial \delta p_{m}}{\partial T_{5}}=-\sum_{n=}^{5 \ldots 7} \frac{\partial p_{n}}{\partial T_{5}} \text { for } m=2,3,4, \\
\frac{\partial \delta e_{m}}{\partial \alpha_{m}}=\frac{\partial e_{m}}{\partial \rho_{m}} \frac{\rho_{m}}{\alpha_{m}} \frac{\partial \delta e_{G}}{\partial \alpha_{m}}=\underbrace{\left(1-\alpha_{0}\right) \sum_{n=1}^{5} x_{n} \frac{\partial e_{n}}{\partial \rho_{n}} \frac{\rho_{n}}{\alpha_{g e}}}_{\text {if } \alpha_{g e}>\alpha_{0}\left(1-\alpha_{1}\right) \text { only; else } 0} \text { for } m=2,3,4 \text {, and } \\
\frac{\partial \delta e_{m}}{\partial T_{m}}=\frac{\partial e_{m}}{\partial T_{m}} \quad \frac{\partial \delta e_{G}}{\partial T_{5}}=\sum_{n=}^{5 \ldots 7} x_{n} \frac{\partial e_{n}}{\partial T_{5}} \quad \text { for } m=2,3,4 .
\end{gathered}
$$

Single-phase mechanical equilibrium: If $\alpha_{\text {Geff }}<\alpha_{0}\left(1-\alpha_{1}\right)$, the requirement is

$$
\begin{aligned}
& \delta p_{m} \equiv p_{m}\left(\rho_{m}, T_{m}\right)-p_{4}\left(\rho_{4}, T_{4}\right)=0 \text { for } m=2,3 \text {, and } \\
& \delta e_{m} \equiv e_{m}\left(\rho_{m}, T_{m}\right)-\bar{e}_{m}=0 \text { for } m=2,3,4
\end{aligned}
$$

where

$$
\begin{gathered}
\rho_{\mathrm{m}}=\frac{\bar{\rho}_{\mathrm{m}}}{\alpha_{\mathrm{m}}} \text { for } \mathrm{m}=2,3,4, \text { and } \\
\alpha_{4}=1-\alpha_{1}-\left(\alpha_{2}+\alpha_{3}\right) .
\end{gathered}
$$

Thus,

$$
\begin{aligned}
& \delta p_{m}\left(\alpha_{2}, \alpha_{3}, T_{m}, T_{4}\right) \equiv p_{m}\left(\frac{\bar{\rho}_{m}}{\alpha_{m}}, T_{m}\right)-p_{4}\left(\frac{\bar{\rho}_{4}}{\alpha_{4}\left(\alpha_{2}, \alpha_{3}\right)}, T_{4}\right)=0 \quad \text { for } m=2,3 \\
& \delta e_{m}\left(\alpha_{m}, T_{m}\right) \equiv e_{m}\left(\frac{\bar{\rho}_{m}}{\alpha_{m}}, T_{m}\right)-\bar{e}_{m}=0 \quad \text { for } m=2,3 \text {, and } \\
& \delta \mathrm{e}_{4}\left(\alpha_{2}, \alpha_{3}, \mathrm{~T}_{4}\right) \equiv \mathrm{e}_{4}\left(\frac{\bar{\rho}_{4}}{\alpha_{4}\left(\alpha_{2}, \alpha_{3}\right)}, \mathrm{T}_{4}\right)-\overline{\mathrm{e}}_{4}=0
\end{aligned}
$$




\section{TABLE VI (CONT.)}

or in matrix notation,

$$
\delta f(x)=0
$$

where

$$
\delta \mathbf{f} \equiv\left(\begin{array}{l}
\delta p \\
\delta \mathrm{e}
\end{array}\right) \equiv\left(\begin{array}{l}
\delta \mathrm{p}_{2} \\
\delta \mathrm{p}_{3} \\
\delta \mathrm{e}_{2} \\
\delta \mathrm{e}_{3} \\
\delta \mathrm{e}_{4}
\end{array}\right) \quad \mathbf{x} \equiv\left(\frac{\alpha}{\mathrm{T}}\right) \equiv\left(\begin{array}{l}
\alpha_{2} \\
\alpha_{3} \\
\mathrm{~T}_{2} \\
\mathrm{~T}_{3} \\
\mathrm{~T}_{4}
\end{array}\right)
$$

This nonlinear matrix equation is solved by a generalized Newton algorithm iterating

$$
\mathbf{x} \leftarrow \mathbf{x}+\delta \mathbf{x}
$$

where

$$
\delta \mathrm{x} \equiv\left(\begin{array}{l}
\delta \underline{\alpha} \\
\delta \mathrm{T}
\end{array}\right) \equiv\left(\begin{array}{c}
\delta \alpha_{2} \\
\delta \alpha_{3} \\
\delta \mathrm{T}_{2} \\
\delta \mathrm{T}_{3} \\
\delta \mathrm{T}_{4}
\end{array}\right)
$$

is calculated from the system of linear equations

$$
\left(\frac{d \delta f}{d x}\right) \delta x=-\delta f
$$

The matrix of this system is

$$
\frac{d \delta f}{d x^{T}} \equiv\left(\begin{array}{ll}
\frac{\partial \delta p}{\partial \alpha^{T}} & \frac{\partial \delta p}{\partial T^{T}} \\
\frac{\partial \delta e}{T \alpha^{T}} & \frac{\partial \delta e}{\partial T^{T}}
\end{array}\right)
$$




\section{TABLE VI (CONT.)}

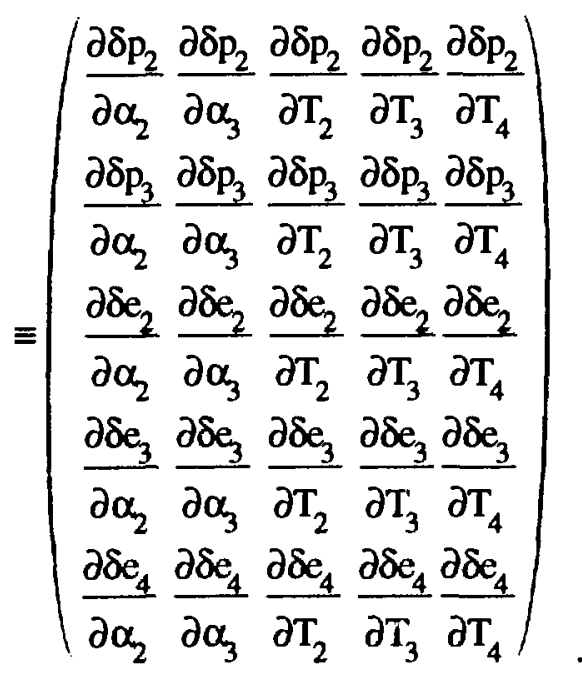

For the two-phase mechanical equilibrium, the order of the system of linear equations to be solved simultaneously can be reduced. Here the elements of the $2 \times 2$ matrix $A$ and of the $2 \times 1$ matrix $b$ are

$$
\begin{aligned}
& \mathrm{a}_{11}=\frac{\partial \delta \mathrm{p}_{2}}{\partial \alpha_{2}}-\frac{\partial \delta \mathrm{p}_{2}}{\partial \mathrm{T}_{2}} \frac{\partial \delta \mathrm{e}_{2}}{\partial \alpha_{2}} / \frac{\partial \delta \mathrm{e}_{2}}{\partial \mathrm{T}_{2}}-\frac{\partial \delta \mathrm{p}_{2}}{\partial \mathrm{T}_{4}} \frac{\partial \delta \mathrm{e}_{4}}{\partial \alpha_{2}} / \frac{\partial \delta \mathrm{e}_{4}}{\partial \mathrm{T}_{4}}, \\
& \mathrm{a}_{12}=\frac{\partial \delta \mathrm{p}_{2}}{\partial \alpha_{3}}-\frac{\partial \delta \mathrm{p}_{2}}{\partial \mathrm{T}_{4}} \frac{\partial \delta \mathrm{e}_{4}}{\partial \alpha_{3}} / \frac{\partial \delta \mathrm{e}_{4}}{\partial \mathrm{T}_{4}}, \\
& \mathrm{a}_{21}=\frac{\partial \delta \mathrm{p}_{3}}{\partial \alpha_{2}}-\frac{\partial \delta \mathrm{p}_{3}}{\partial \mathrm{T}_{4}} \frac{\partial \delta \mathrm{e}_{4}}{\partial \alpha_{2}} / \frac{\partial \delta \mathrm{e}_{4}}{\partial \mathrm{T}_{4}}, \\
& \mathrm{a}_{22}=\frac{\partial \delta \mathrm{p}_{3}}{\partial \alpha_{3}}-\frac{\partial \delta \mathrm{p}_{3}}{\partial \mathrm{T}_{3}} \frac{\partial \delta \mathrm{e}_{3}}{\partial \alpha_{3}} / \frac{\partial \delta \mathrm{e}_{3}}{\partial \mathrm{T}_{3}}-\frac{\partial \delta \mathrm{p}_{3}}{\partial \mathrm{T}_{4}} \frac{\partial \delta \mathrm{e}_{4}}{\partial \alpha_{3}} / \frac{\partial \delta \mathrm{e}_{4}}{\partial \mathrm{T}_{4}}, \\
& \mathrm{~b}_{1}=-\delta \mathrm{p}_{2}+\frac{\partial \delta \mathrm{p}_{2}}{\partial \mathrm{T}_{2}} \delta \mathrm{e}_{2} / \frac{\partial \delta \mathrm{e}_{2}}{\partial \mathrm{T}_{2}}+\frac{\partial \delta \mathrm{p}_{2}}{\partial \mathrm{T}_{4}} \delta \mathrm{e}_{4} / \frac{\partial \delta \mathrm{e}_{4}}{\partial \mathrm{T}_{4}}, \text { and } \\
& \mathrm{b}_{2}=-\delta \mathrm{p}_{3}+\frac{\partial \delta \mathrm{p}_{3}}{\partial \mathrm{T}_{3}} \delta \mathrm{e}_{3} / \frac{\partial \delta \mathrm{e}_{3}}{\partial \mathrm{T}_{3}}+\frac{\partial \delta \mathrm{p}_{3}}{\partial \mathrm{T}_{4}} \delta \mathrm{e}_{4} / \frac{\partial \delta \mathrm{e}_{4}}{\partial \mathrm{T}_{4}},
\end{aligned}
$$

and the elements of the $3 \times 2$ matrix $C$ and of the $3 \times 1$ matrix $d$ are (nonzero elements only) 


\section{TABLE VI (CONT.)}

$$
\begin{aligned}
& c_{11}=-\frac{\partial \delta \mathrm{e}_{2}}{\partial \alpha_{2}} / \frac{\partial \delta \mathrm{e}_{2}}{\partial \mathrm{T}_{2}}, \\
& c_{22}=-\frac{\partial \delta \mathrm{e}_{3}}{\partial \alpha_{3}} / \frac{\partial \delta \mathrm{e}_{3}}{\partial \mathrm{T}_{3}}, \\
& c_{31}=-\frac{\partial \delta \mathrm{e}_{4}}{\partial \alpha_{2}} / \frac{\partial \delta \mathrm{e}_{4}}{\partial \mathrm{T}_{4}}, \\
& c_{32}=-\frac{\partial \delta \mathrm{e}_{4}}{\partial \alpha_{3}} / \frac{\partial \delta \mathrm{e}_{4}}{\partial \mathrm{T}_{4}}, \\
& \mathrm{~d}_{1}=-\partial \delta \mathrm{e}_{2} / \frac{\partial \delta \mathrm{e}_{2}}{\partial \mathrm{T}_{2}}, \\
& \mathrm{~d}_{2}=-\partial \delta \mathrm{e}_{3} / \frac{\partial \delta \mathrm{e}_{3}}{\partial \mathrm{T}_{3}}, \text { and } \\
& \mathrm{d}_{3}=-\partial \delta \mathrm{e}_{4} / \frac{\partial \delta \mathrm{e}_{4}}{\partial \mathrm{T}_{4}} .
\end{aligned}
$$

The derivatives in the above matrix elements are calculated using the basic derivatives of $p_{m}$ and $e_{m}$ with respect to $\rho_{m}$ and $T_{m}$ :

$$
\begin{gathered}
\frac{\partial \delta p_{m}}{\partial \alpha_{m^{\prime}}}=-\underbrace{\frac{\partial p_{m}}{\partial \rho_{m^{\prime}}} \frac{\rho_{m^{\prime}}}{\alpha_{m^{\prime}}}}_{\text {if } m=m^{\prime} \text { only }}-\frac{\partial p_{4}}{\partial \rho_{4}} \frac{\rho_{4}}{\alpha_{4}} \quad \text { for } m=2,3, m^{\prime}=2,3, \\
\frac{\partial \delta p_{m}}{\partial T_{m}}=\frac{\partial p_{m}}{\partial T_{m}} \quad \frac{\partial \delta p_{m}}{\partial T_{4}}=-\frac{\partial p_{4}}{\partial T_{4}} \quad \text { for } m=2,3, \\
\frac{\partial \delta e_{m}}{\partial \alpha_{m}}=-\frac{\partial e_{m}}{\partial \rho_{m}} \frac{\rho_{m}}{\alpha_{m}} \quad \frac{\partial \delta e_{4}}{\partial \alpha_{m}}=\frac{\partial e_{4}}{\partial \rho_{4}} \quad \text { for } m=2,3 \text {, and } \\
\frac{\partial \delta e_{m}}{\partial T_{m}}=\frac{\partial e_{m}}{\partial T_{m}} \quad \text { for } m=2,3,4 .
\end{gathered}
$$




\section{TABLE VI (CONT.)}

The incompressible component $\mathrm{m}=1$ does not take part in the mechanical equilibriam. Thus, the temperature $T_{1}$ is calculated from

$$
\delta e_{1} \equiv e_{1}\left(T_{1}\right)-\bar{e}_{1}=0
$$

by a Newton algorithm iterating

$$
\mathrm{T}_{1} \leftarrow \mathrm{T}_{1}-\frac{\delta \mathrm{e}_{1}}{\frac{\mathrm{d} \delta \mathrm{e}_{1}}{\mathrm{dT} \mathrm{T}_{1}}}
$$

where

$$
\frac{\mathrm{d} \delta \mathrm{e}_{1}}{\mathrm{dT}}=\frac{\mathrm{de}_{1}}{\mathrm{dT}_{1}}
$$

\section{E. The TEOS Access, Search, and Interpolation Routines}

1. Introduction. In this chapter, the lower-level routines of the TEOS interface are described, i.e., the routines that are called by the interface routines proper to obtain the thermodynamic equilibrium properties and/or their derivatives from the tables for given arguments. These routines are included in the compilable code only if the SESAME option has been specified at the time of preprocessing the source code. All routines are vectorized to sweep over the pertaining cells of the mesh. For the specifications of the routines, see App. B.

To help in understanding these routines, a description of the storage arrays where the TEOS data of the materials needed by the code are kept and of other variables related to these tables is included. To start with, an overview of the SESAME EOS data library is given.

2. The SESAME EOS Data Library. The SESAME EOS Library is a standardized, computer-based library of tables of thermodynamic properties and FORTRAN subroutines developed by the former "Equation-of-State and Opacity Group of the Theoretical Division of LANL." 2 
Routine:

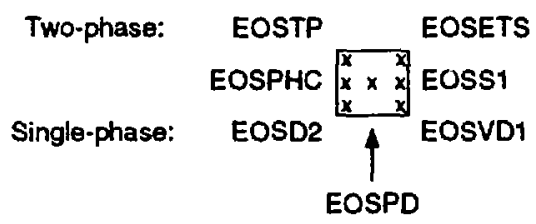

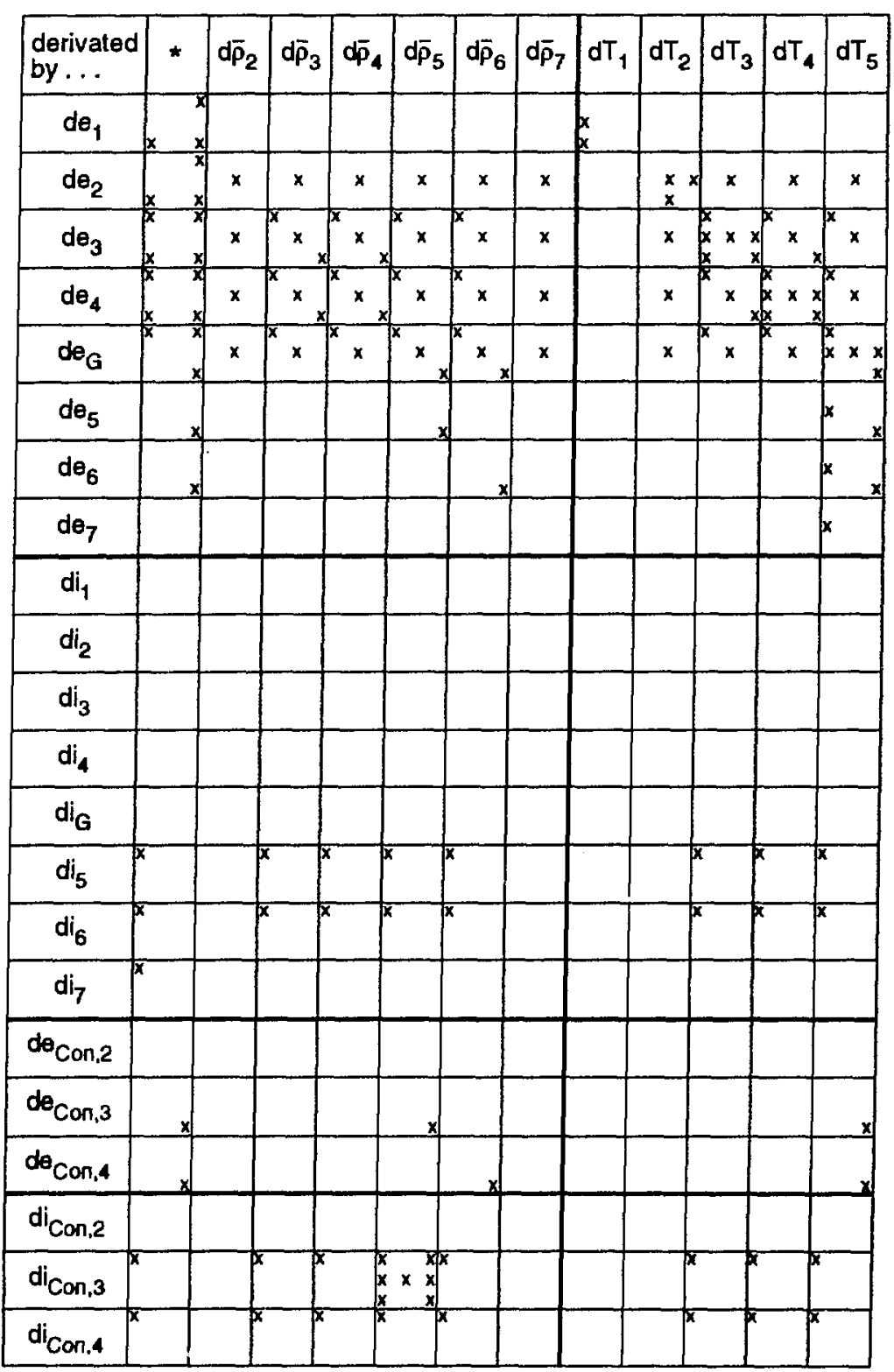

*This column indicates the calculation of the underivated quantities $e_{1}, \bullet_{2}, \ldots$

Fig. 3.12.

Calculation of the EOS property derivatives. 
Routine:

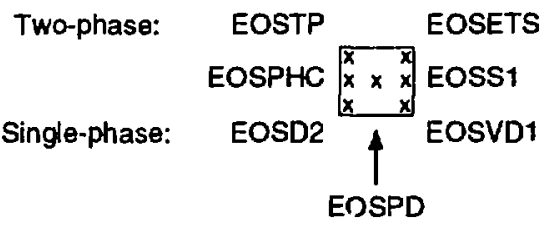

\begin{tabular}{|c|c|c|c|c|c|c|c|c|c|c|c|c|}
\hline $\begin{array}{l}\text { derivated } \\
\text { by... }\end{array}$ & $\star$ & $\mathrm{d} \bar{\rho}_{2}$ & $d \bar{\rho}_{3}$ & $d \bar{\rho}_{4}$ & $d \bar{\rho}_{5}$ & $d \bar{p}_{6}$ & $d \bar{p}_{7}$ & $d T_{1}$ & $d T_{2}$ & $d T_{3}$ & $d T_{4}$ & $d T_{5}$ \\
\hline \multicolumn{13}{|l|}{$\mathrm{de}_{\mathrm{Vap}, 2}$} \\
\hline \multicolumn{13}{|l|}{$d e_{\text {Vap. } 3}$} \\
\hline \multicolumn{13}{|l|}{$d e_{V a p, 4}$} \\
\hline \multicolumn{13}{|l|}{$\mathrm{di}_{\text {Vap,2 }}$} \\
\hline \multicolumn{13}{|l|}{$\mathrm{di}_{\mathrm{Vap}, 3}$} \\
\hline \multicolumn{13}{|l|}{$\mathrm{di}_{\text {Vap,4 }}$} \\
\hline \multicolumn{13}{|l|}{$d e_{\mid g, 2}$} \\
\hline \multicolumn{13}{|l|}{$d \theta_{1 g, 3}$} \\
\hline \multicolumn{13}{|l|}{$d \theta_{\mid g, 4}$} \\
\hline \multicolumn{13}{|l|}{$\mathrm{dh}_{\mathrm{lg}, 2}$} \\
\hline \multicolumn{13}{|c|}{\begin{tabular}{l|l|l}
$d h_{\lg , 3}$ & $x$ \\
\end{tabular}} \\
\hline \multicolumn{13}{|l|}{\begin{tabular}{l|l}
$d h_{1 g, 4}$ & $x$ \\
\end{tabular}} \\
\hline \multicolumn{13}{|l|}{$\mathrm{dT}_{\text {Sat,2 }}$} \\
\hline \multicolumn{13}{|l|}{\begin{tabular}{|l|l}
$\mathrm{dT}_{\text {Sat,3 }}$ & $\begin{array}{l}\mathrm{x} \\
\mathrm{x}\end{array}$ \\
\end{tabular}} \\
\hline \multicolumn{13}{|c|}{ dT $\left.\mathrm{Sat}_{\text {Sa }}\right]_{x}^{\mathrm{x}}$} \\
\hline \multicolumn{13}{|l|}{$d p_{\text {Sat,2 }}$} \\
\hline \multicolumn{13}{|c|}{$\mathrm{dp}_{\mathrm{Sat}, 3} \times$} \\
\hline \multicolumn{13}{|l|}{$\mathrm{dp}_{\mathrm{Sal}, 4} \mathrm{x}^{\mathrm{x}}$} \\
\hline$d p$ & & $x$ & $x$ & $x$ & $x$ & $x$ & $x$ & & $x$ & $x$ & $x$ & $x$ \\
\hline$d \alpha$ & & $x$ & $x$ & $x$ & $x$ & $x$ & $x$ & & $x$ & $x$ & $x$ & $x$ \\
\hline
\end{tabular}

${ }^{\star}$ This column indicates the calculation of the underivated quantities $e_{1}, e_{2}, \ldots$

Fig. 3.12 (Cont.).

Calculation of the EOS property derivatives. 
Of the multitude of materials and properties contained in the LANL SESAME EOS Data Library, only a subset is needed by the AFDM code. It is contained in the SESAMESO library, which is exported along with the AFDM code. The SESAMESO library contains data for the fuel, steel, and sodium substances and the properties listed below. Some table entries have been added in generating the SESAMESO library, as compared to the original SESAME library (see initial footnote in App. F).

In its portable form, a library in the SESAME format consists of the tabular data and of routines to construct a file at the user's computing facility from these data. For the details of this process and of the file structure, see Vol. VII, App. H, of the AFDM documentation.

The subroutines for accessing the SESAME file were taken from the EOSPAC subroutine package ${ }^{6}$ and were adapted to and included in the AFDM code. They access the library data in two phases. During the initialization phase, the requested data tables are read into a storage area and are converted to a form suitable for the AFDM code. During the transient phase, the stored data tables are used to compute the EOS values required by the AFDM code. The format of the storage area, and the specifications of the access routines are described in the next sections.

The materials in the SESAME file are identified by a five-digit number. For each material, there are several EOS data types identified by catalog numbers. In the AFDM code, only two data types are fetched from the SESAME file: total pressure and specific energy (catalog number 301); and saturation properties (catalog number 401). In the SESAME file, these quantities are not in SI units and thus must be converted to SI units during the initialization phase.

In the 301 tables of the SESAME library, the pressure and the specific internal energy are tabulated over the density and the temperature. The full Van-der-Waalstype loops are tabulated irrespective of saturation and spinodal limits. In the 401 tables, the saturation pressure, saturated vapor and condensated liquid densities and internal energies are tabulated over the temperature. In App. F, data of the quantities are shown for the fuel, steel, and sodium materials of the current SESAMESO library. The order of the materials in the SESAME file (to which the order in the MATER array must correspond) determines the order in the storage tables (see Sec. III.E.4).

Unconnected to the SESAME library are the solid EOS and sublimation data of the substances. Fuel and steel tables in the SESAME format, which contain data compatible with the related Van-der-Waals-type data in the SESAME library, have been provided by E. A. Fischer. ${ }^{11}$ These tables have been included in the 
SESAMESO library as separate materials with a special identification. The solid EOS is treated as type-301 data, and the sublimation data is treated as type-401 data. Note that no solid EOS and sublimation tables for sodium are provided and that the solid steel tables contain dummy data (see App. F). Finally, note that although solid steel data have been provided, the substance Solid 2 need not be identical with the substance Liquid/Vapor 2.

3. Other EOS Data Tables. In addition to the above data, the AFDM code needs the spinodal data derived from the 301 data of the materials. These data are not provided by the SESAME library. The code includes an option that can be invoked by an input parameter to produce tables with these data from the 301 data during the initialization phase. The spinodal data of fuel, steel, and sodium derived from the current version of the 301 data are also contained as default input tables in the AFDM code (see App. F; note that the order in the tables is "sodium, steel, fuel," corresponding to the default material assignment).

4. Storage of the Data Tables. During the initialization phase, the AFDM code extracts the data tables for the materials needed from the SESAMESO file and stores them in COMMON arrays. The arrays have been dimensioned such that equal space is allotted to all materials. To minimize the storage region occupied by the AFDM code, the sizes of the largest entries of the material data tables should determine the array dimensions. ${ }^{*}$ The current dimensions are:

maximum number of materials: $\mathrm{MREGS}=5$, maximum number of density entries in 301 tables: $\operatorname{MAXR3}=150$, maximum number of temperature entries in 301 tables: MAXT3 $=150$, maximum number of temperature etc. entries in 401 tables: MAXT4 $=100$, and

maximum number of temperature etc. entries in spinodal tables: NSPIN $=16$.

After the actual number of materials SESNM and material identifiers [MATER $(1, \ldots$, SESNM) of the MATER array, dimension MREGS] have been specified by explicit or default input, the actual sizes of the tables are loaded from the SESAMESO file into the following arrays (dimension MREGS):

\footnotetext{
*This currently is not the case, as can be seen from App. F. To minimize the region, the values of MAXT3 and MAXT4 should be overridden by 50,102 , and 38 in the XEOSDIM comdeck of the code source.
} 
actual number of density entries in 301 tables: NR3(1,..,SESNM), actual number of temperature entries in 301 tables: NT3(1,.,,SESNM), actual number of temperature etc. entries in 401 tables: NT4 $(1, \ldots$, SESNM), and

actual number of temperature etc. entries in spinodal tables:

NSPNDL $(1, \ldots$, SESNM)

The values for the default input substances fuel, steel, and sodium of the SESAMESO library are listed in App. F.

The 301 tables are loaded from the SESAMESO library (by the SESINI routine) into the EOSRO (densities $\rho$ ), EOSTE (temperatures T), EOSPR (pressures pvan $\{\rho, T\}$ or PSId $\{\rho, T\}$ ), and EOSEN (specific internal energies $e_{v a n}\{\rho, T\}$ or esld $\{\rho, T\}$ ) arrays. These arrays are equivalenced to the EOSTB array, as shown in Fig. 3.13. Note that in the EOSPR and EOSEN arrays, the data are stored densely and the order of the entries is determined by the $\rho$ argument varying fastest.

The 401 tables are loaded from the SESAMESO library (again by the SESINI routine) into the SATT (temperatures T), SATP (saturation pressures PSat $\{$ T $\}$ or sublimation pressures PSus $\{T\}$ ), SATRG (saturated vapor densities $\rho$ Vap $\{T\}$ or solid densities at sublimation $\left.\rho_{S u s}(T\}\right)$, SATRL (condensated liquid densities $\rho_{C}$ Con $\{T\}$ ), SATEG (saturated vapor specific internal energies evap $\{\mathrm{T}\}$ ) or solid specific internal energies at sublimation esus $\{\mathrm{T}\}$ ), and SATEL (condensated liquid specific internal energies econ $(T\})$ arrays. These arrays are equivalenced to the SATTB array, as shown in Fig. 3.14.

The spinodal data tables are initialized with default values (see Sec. II.E.3)which can be overridden by data derived from the SFSAMESO library (by the SPNINI routine)-in the SPNT (temperatures T), SPNI'G (vapor spinodal limit pressures PSpg $\{\mathrm{T}\}$ ), SPNPL (liquid spinodal limit pressures PSpI(T\}), SPNRG (vapor spinodal limit density $\rho_{S p g}\{T\}$ ), and SPNRL (liquid spinodal limit density $\rho_{S p l}(T\}$ ) arrays. These arrays are equivalenced to the SPNDL array, as shown in Fig. 3.15.

The last entry in the arrays of both the saturation and spinodal tables corresponds to the critical point (critical temperature $T_{\mathrm{Crt}}$, critical pressure $\mathrm{PCrt}$, critical density $\rho C r$, and critical specific internal energy $e_{C r}$ ).

5. Tables Search and Interpolation. During the transient phase of the AFDM code, the data tables of the previous section must be interpolated frequently 


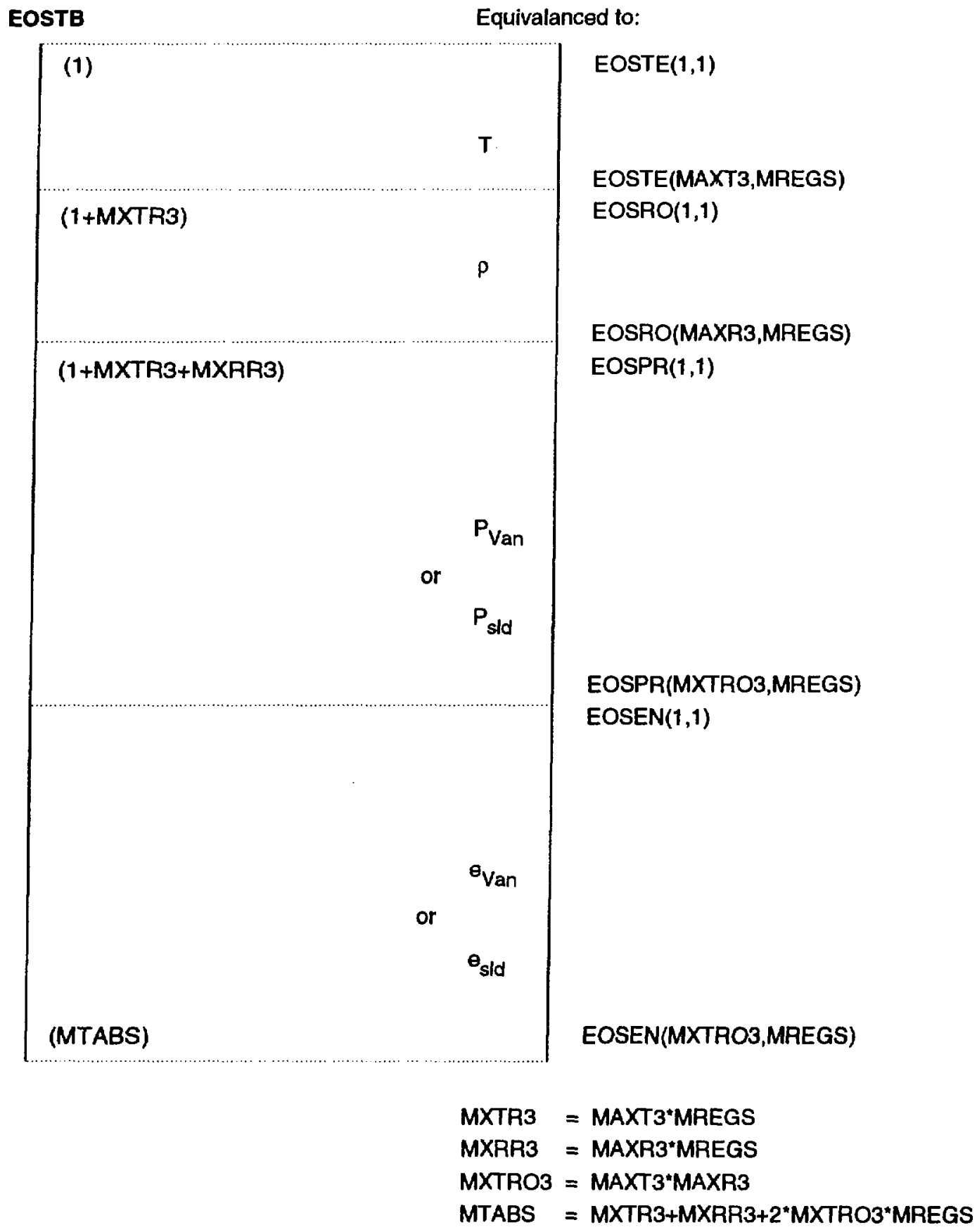

Fig. 3.13.

EOSTB array (Van-der-Waals-type data). 
SATTB

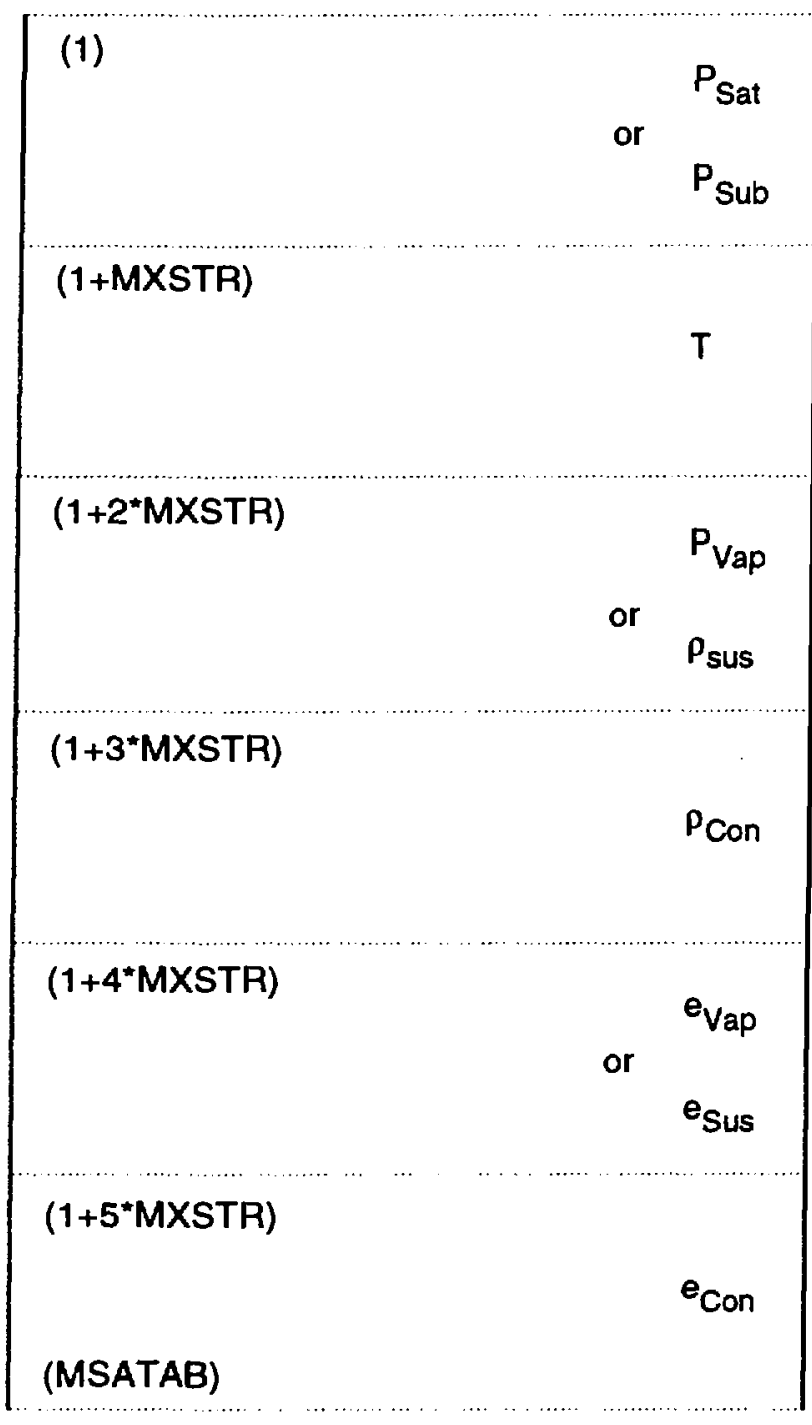

Equivalanced to:

$$
\begin{aligned}
& \text { SATP }(1,1) \\
& \text { SATP(MAXT4,MREGS) } \\
& \text { SATT }(1,1)
\end{aligned}
$$

SATT(MAXT4,MREGS)

$\operatorname{SATRG}(1,1)$

SATRG(MAXT4,MREGS)

$\operatorname{SATRL}(1,1)$

SATRL(MAXT4,MREGS)

$\operatorname{SATEG}(1,1)$

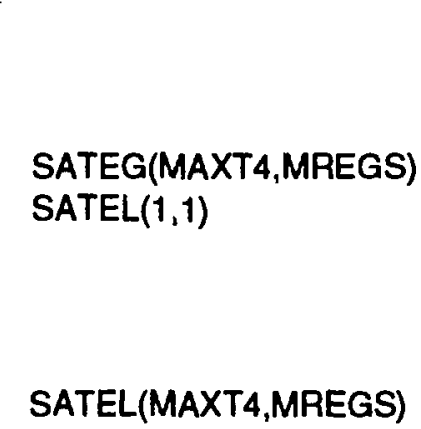

$$
\begin{aligned}
\text { MXSTR } & =\text { MAXT4 } 4^{*} \text { MREGS } \\
\text { MSATAB } & =6^{*} \text { MXTR }
\end{aligned}
$$

Fig. 3.14.

SATTB array (saturation data). 


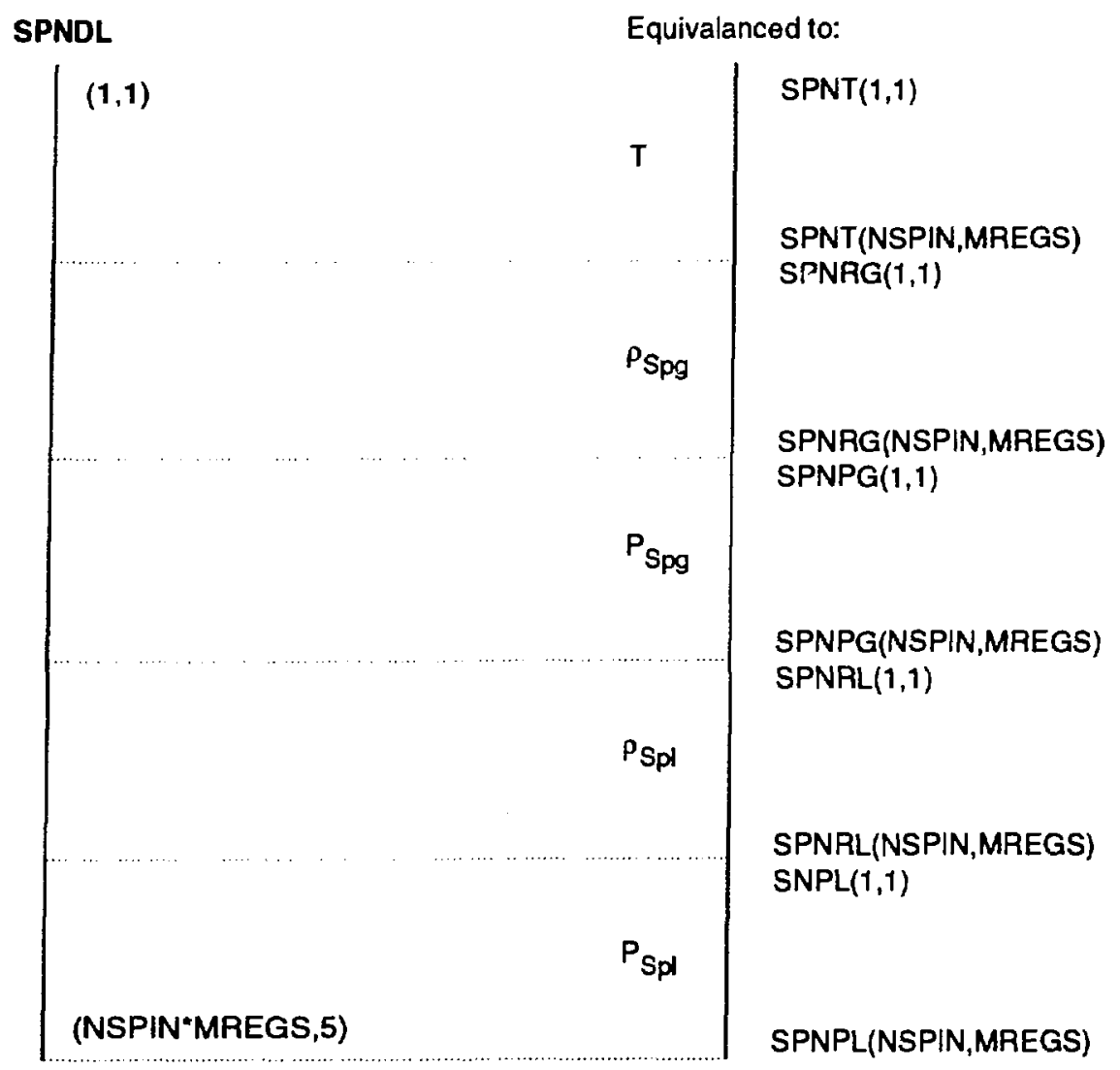

Fig. 3.15.

SPBDL array (spinodal data).

for given $T$ - and $\rho$ arguments to return values and derivatives. Several interpolation methods are applied, as described below. Before the interpolation can be performed, the interpolating points of the pertaining interval must be found. For this purpose, the code routines use a linear search.

The interpolation method for the 301 tables can be selected by requiring input to be either bilinear, biquadratic, or birational (linear, quadratic, or rational, respectively, at the table boundaries). The routines from the EOSPAC subroutine package 6 are used for these interpolations; they are called by the SESEOS routine.*

\footnotetext{
* Note that the EOSPAC routines return the logarithms of the derivatives instead of the derivatives proper. To get the derivatives, the logarithms must be divided by the independent variable. Because there is no meaning behind this detour (as can be seen from the EOSPAC routine coding), the superfluous multiplications and divisions must be eliminated.
} 
The interpolation method for the 401 and spinodal data tables is fixed to a logarithmic/reciprocal-type interpolation or a linear interpolation, depending on the quantities to be interpolated. The URANUS and LAGRAN routines, respectively, are called for this purpose by the VAPDOM routine in the case of saturation data, by the VSTOP, VSPOT, VSELOT, VSTOEL routines in the case of 401-type data, and by the SPINDM routine in the case of spinodal data.

6. Initialization Routines SESINI, SPNINI, and SESPRT. Ii the SESAME option has been set "on" at the time of preprocessing the source code, the SESINI and the SPNINI routines are both called (through the EOSUP routine) by the INIPAR routine during the initialization phase of the code. SESINI loads the required SESAMESO tables into the storage arrays, and SPNINI generates spinodal data (if requested by input) and loads these into storage to override the default data there. The SESPRT routine is called under this option by the INIPRT routine (if requested by input) to print the stored tables.

\section{Access Routines SESEOS, VSTOP, VSPOT, VSELOT, VSTOEL, and} SPINDM. The SESEOS, VSTOP, VSPOT, VSELOT, VSTOEL, and SPINDM routines are called frequently by the TEOS interface routines. They access the Van-der-Waalstype and solid EOS, saturation and sublimation, and spinodal tables, respectively, in the code storage arrays to return table values and their derivatives for given arguments.

8. EOSPAC Search and Interpolation Routines. Search and interpolation in the Van-der-Waals-type and solid EOS tables is done by routines from the EOSPAC subroutine package, ${ }^{6}$ which is called by the SESEOS routine. For each of the three interpolation methods (see Sec. III.E.5), a table search routine, an interpolation routine for points inside the two-dimensional table, and an interpolation routine for points on the table boundaries are included in the code:

SRCHDF, BILIN, LINEAR,

SRCH2D, BIQUAD, QUAD, and

SRCH3D, BIRATF, RATF.

For details on these routines, the reader is referred to the EOSPAC documentation $^{6}$ and to the comments in the coding of these routines.

9. Search and Interpolation Routines LAGRAN and URANUS. Search and interpolation in the saturation, spinodal, and sublimation tables is done by the LAGRAN and URANUS routines. The former interpolates lineariy, the latter 
interpolates logarithmically/reciprocally (see Sec. III.E.5). The LAGंRAN and URANUS routines are called by the VSTOP, VSPOT, VSELOT, VSTOEL, and SPINDM routines for the saturation, spinodal, and sublimation tables. 


\section{THERMOPHYSICAL PROPERTIES}

In the model for the intracell exchanges of AFDM, step 1, thermophysical properties such as surface tension, thermal conductivities, and viscosities are needed. An analytical form is chosen for these properties, the complexity of which depends on the data available and/or the precision assumed to be needed for the models. The figures and tables referred to in this chapter are part of App. G.

\section{A. Surface Tension}

There are three surface tensions: between liquid fuel and vapor, between liquid coolant and vapor, and between liquid fuel and liquid coolant. Because of considerable complexity and lack of consistent data, the influence of the composition of the vapor phase on the surface tensions has been neglected, and a twoequation form has been chosen, depending on the interface temperature. Data and plots are given for $\mathrm{UO}_{2}$ as fuel, stainless steel as coolant, and both $\mathrm{UO}_{2}$ and stainless steel vapor phases as corresponding vapors. The parameters used in this representation are input parameters to the code. All surface tensions have a finite value beyond the critical point to accommodate any future models with artificial supercritical states. All surface tensions are calculated in subroutine INIFA. References 12, $13,14,15,16$, and 17 are considered while choosing the following correlations.

1. General Form of the Surface Tension Correlation. The surface tension $\sigma$, given in $\mathrm{kg} / \mathrm{s}^{2}$, is defined for the liquid-vapor and liquid-liquid interfaces. The input parameter is the interface temperature $\mathrm{T}^{\mathrm{I}}$ and the property parameters.

a. Liquid-vapor surface tension. The liquid-vapor surface tension is given by two equations,

$$
\begin{array}{ll}
\sigma_{L G}=\sigma_{L N}+\sigma_{L F L} T^{I} & \text { for } T^{I} \leq T_{\sigma L C}, \text { and } \\
\sigma_{L G}=\sigma_{L F E} \max \left(0.3,1-T^{I} / T_{C r t}\right)^{\sigma L E X} & \text { for } T^{I}>T_{\sigma L C},
\end{array}
$$

and where $T_{\sigma L C}$ is the temperature to specify the two domains that are an input parameter, as well as $\sigma_{\mathrm{LN}}, \sigma_{\mathrm{LFL}}, \sigma_{\mathrm{LFE}}$, and $\sigma_{\mathrm{LEX}}$. Table G.I (App. G) lists the default values used in AFDM.

b. Liquid-liquid surface tension. The liquid-liquid surface tension is given by

$$
\sigma_{L L}=\sigma_{L L I N}+\sigma_{L L I F} \max \left(0, T_{S L L I R}-T^{\mathrm{I}}\right)^{2}
$$


where TSLLIR is the temperature at the transition between the quadratic function and the constant value that is an input parameter, as well as $\sigma_{\text {LLIN }}$ and $\sigma_{\text {LLIF. }}$ Table G.II (App. G) lists the default values used in AFDM.

2. Surface Tension Function. The three surface tensions using the default values are plotted in Fig. G.1. Besides referring to the values directly in the step 1 models, a surface tension function is needed to assess the three-component contact. The function has the form

$$
\sigma_{\mathrm{GLm}}-\sigma_{\mathrm{GLn}}-\sigma_{\mathrm{LmLn}}
$$

and Fig. G.2 shows the values for the given default parameters, using the subscripts $\mathrm{GL} 1=\mathrm{UO}_{2}, \mathrm{GL} 2=\mathrm{SS}$, and L1L2 $=\mathrm{SS}-\mathrm{UO}_{2}$. Applying the AFDM model for the three-component constant in topologies 11 and 12, a vapor bubble is likely to have intimate contact with both liquid phases if both surface tension functions are negative, whereas a vapor bubble tends to have exclusive contact in all other cases, where the liquid partner is chosen by the values of the surface tension functions. If the first value of Fig. G.2 is positive, the bubbles have intimate contact with liquid $1=\mathrm{UO}_{2}$; if the second value is positive, the bubbles have intimate contact with liquid 2 = stainless steel.

\section{B. Vapor Thermophysical Properties}

If the KQQM1 option is on, the thermal conductivities are input constants. If the KQQM2 option is on, the vapor thermal conductivity and viscosity are represented using the Lennard-Jones parameters. Because the theory predicts a close relationship between both properties, these properties are always calculated together. The main parameter for some of the following correlations is the bulk temperature $\mathrm{T}$ of the given component. The vapor thermophysical properties are calculated in subroutine THEPHY.

1. Heat Capacity at Constant Pressure. The heat capacity at constant volume $c_{v}$ is given by the EOS. For ideal gases, the heat capacity at constant pressure $c_{p}$ is

$$
c_{p}=c_{v}+R
$$

where $\mathrm{R}$ is the gas constant of the given vapor component. As the state of the gas approaches the vapor dome, the representation by a constant $R$ no longer holds. However, the heat capacity at constant pressure $c_{p}$ is used only in defining the 
Nusselt and Prandtl numbers. Therefore, the approximation here should be sufficient.

2. Vapor Dynamic Viscosity. The general approach for dilute gases is to use the corresponding-states method; see Refs. 18, 19, and 20. The dynamic viscosity $\mu$ calculated in $\mathrm{kg} / \mathrm{m}-\mathrm{s}$ is now

$$
\mu=2.67 \cdot 10^{-6} \sqrt{\mathrm{MT}} /\left(\sigma^{2} \Omega_{\mu}\right) \text {, }
$$

where the molecular diameter, $\sigma$, is given in $10^{-10} \mathrm{~m}=1 \dot{\mathrm{A}}$, and the collision integral $\Omega_{\mu}$ is a weak function of the gas bulk temperature T. A simple approximation of Ref. 15 is used with

$$
\begin{aligned}
\Omega_{\mu} & =0.92495+2.07368 \times 10^{-3} \frac{\mathrm{T}}{\varepsilon / \mathrm{k}}+0.719288\left(\frac{\mathrm{T}}{\varepsilon / \mathrm{k}}\right)^{-1.15049} \\
& -5.46452 \times 10^{-2}\left(\frac{\mathrm{T}}{\varepsilon / \mathrm{k}}\right)^{0.5}
\end{aligned}
$$

The Lennard-Jones parameter $\varepsilon / k$, which is the characteristic energy of interaction between molecules over the Boltzmann constant, must be given as an input parameter for each component.

3. Vapor Thermal Conductivity. The thermal conductivity for monoatomic phases, $k_{\text {mono, }}$ is a function of the dynamic viscosity, as given by Ref. 21 . For $n_{A}=1$, where $n_{A}$ is the number of atoms per gas molecule, which is an input constant:

$$
\mathrm{k}_{\text {mono }}=\frac{5}{2} \mathrm{c}_{\mathrm{v}} \mu
$$

Reference 16 proposed a modified Eucken correlation to compute the conductivity of polyatomic gases with

$$
\mathrm{k}=\mathrm{k}_{\text {mono }}+1.32 \mu\left(\mathrm{c}_{\mathrm{v}}-\frac{3 \mathrm{R}^{\circ}}{2 \mathrm{M}}\right)
$$

where $R^{\circ}$ is the universal gas constant.

4. Vapor Mixture Rules. As given by Ref. 17, the mixture rules for the viscosity and the thermal conductivity of gases are 


$$
\begin{aligned}
& \mu_{\mathrm{a}}=\sum_{\mathrm{m}=1}^{3} \frac{\mathrm{Y}_{\mathrm{m}} \mu_{\mathrm{m}}}{\sum_{k=1}^{3} \mathrm{Y}_{\mathrm{i}} \Psi_{\mathrm{mi}}} \text {, and } \\
& \mathrm{k}_{\mathrm{a}}=\sum_{\mathrm{m}=1}^{3} \frac{\mathrm{Y}_{\mathrm{m}} \mathrm{k}_{\mathrm{m}}}{\sum_{\mathrm{k}=1}^{3} \mathrm{Y}_{\mathrm{i}} \Psi_{\mathrm{mi}}}
\end{aligned}
$$

when the mole fraction of vapor component $m$ is given by

$$
Y_{m}=\frac{\rho_{\mathrm{Gm}} / M_{m}}{\sum_{k=1}^{3} \bar{\rho}_{\mathrm{Gi}} / M_{i}}
$$

and the coefficient $\psi_{\mathrm{mi}}$ is a function of the individual viscosities and molecular weights with

$$
\psi_{\mathrm{mi}}=\frac{\left\{1+\left[\mu_{\mathrm{m}} / \mu_{\mathrm{i}}\left(\mathrm{M}_{\mathrm{l}} / \mathrm{M}_{\mathrm{m}}\right)^{0.5}\right]^{0.5}\right\}^{2}}{\left(8+8 \mathrm{M}_{\mathrm{m}} / \mathrm{M}_{1}\right)^{0.5}}
$$

If $\mathrm{m}=\mathrm{i}$, then $\Psi_{\mathrm{mi}}=1$.

5. Second-Order Correlation for the Thermal Conductivity. If the thermal conductivity of a vapor component is known by experimental data or other reliable sources, a second-order polynomial can be used to override the theoretical value, as given in Sec. IV.B.3:

$$
k=C_{K A G}+C_{K B G} T+C_{K C G} T^{2}
$$

The default values for input parameters $C_{K A G}, C_{K B G}$, and $C_{K C G}$ are zero, for which the theoretical value is used instead of the polynomial.

6. Mixture Heat Capacity. The heat capacity at constant pressure for a gas mixture is given for all compiler options by

$$
c_{\mathrm{pmix}}=\sum_{m=1}^{3} c_{\mathrm{pm}} \bar{\rho}_{\mathrm{m}} / \sum_{\mathrm{m}=1}^{3} \bar{\rho}_{\mathrm{m}},
$$


where $\mathrm{c}_{\mathrm{pm}}$ is the heat capacity at constant pressure of vapor component $\mathrm{m}$, as calculated using the correlation of Sec. IV.B.1.

\section{Solid and Liquid Thermophysical Properties}

If the KQQM1 option is on, the liquid viscosities and the solid and liquid thermal conductivities are input constants. If the KQQM2 option is on, the liquid viscosities and solid and liquid thermal conductivities are given in the form of simple correlations. The user must define input parameters, but default values for $\mathrm{UO}_{2}$ and stainless steel are provided by the code. Plots of the properties using the default values are shown in Figs. G.3 through G.7. The main parameter is the bulk temperature $T$ of the given component. The solid and liquid thermophysical properties are calculated in subroutine THEPHY.

1. Liquid Dynamic Viscosity. The general form for the liquid viscosity is

$$
\mu=C_{M U A} \exp \left[C_{M U B} / \max \left(T, T_{L i q}\right)\right],
$$

where $T_{L i q}$ is the liquidus temperature and $C_{M U A}$ and $C_{M U B}$ are input parameters, the default values of which are listed in Table G.III. The default vaiues were chosen using Refs. 19 and 22. $\mathrm{T}_{\text {Liq }}$ is given by the EOS. The plots in Figs. G.3 and G.4 range from $\mathrm{T}_{\mathrm{Liq}}$ to $\mathrm{T}_{\mathrm{Crt}}$.

2. Solid and Liquid Thermal Conductivity. AFDM material 1 can exist in all three phases, whereas material 2 can exist only as liquid and vapor. Therefore, the thermal conductivity must be defined for solid particulate of material 1, the structure component, liquid 1, and liquid 2. The general form for the thermal conductivity is

$$
k=C_{K A}+C_{K B} T \text {, }
$$

where $C_{K A}$ and $C_{K B}$ are input parameters. The default values are listed in Table G.4 and were chosen using Refs. 16 and 19. The structure value is supposed to be constant because of the lack of a detailed structure model. Although the structure cannot melt, data in Fig. G.5 are shown up to $T_{\text {Liq. }}$. However, the code may calculate values beyond this limit. Figure G.6 shows liquid stainless-steel values between $\mathrm{T}_{\mathrm{Liq}}$ and $\mathrm{T}_{\mathrm{Crt}}$. Figure $\mathrm{G} .7$ shows the $\mathrm{UO}_{2}$ values for both phases. 


\section{ACKNOWLEDGEMENTS}

During the development of the code, the EOS formalism was repeatedly modified because initial treatments of the SESAME interface proved to be inadequate and models were developed with new EOS requirements. Several authors contributed to designing, specifying, revising, coding, debugging, testing, improving, and documenting the EOS part of the AFDM code, and no routine was in the hands of one and the same author through all these stages of the code development. At present, the EOS implementation is not optimized. In view of potential future code developments on the basis of AFDM, inconsistencies showing up in the context of the documentation of the EOS are pointed out in comments and footnotes in this volume.

Except for F. Parker, who was responsible for the final coding approach, all contributors to the EOS part of the code are represented in the following list of authors of this volume: Chap. I: S. Kleinheins; Chap. II: W. Bohl; Chap. III.B, originally written by $\mathrm{P}$. Maudlin, rewritten by $\mathrm{H}$. Ninokata and L. Goutagny, revised and extended by E. A. Fischer; Chap. III.C (except for minor corrections and additions by the compilers): L. Goutagny; Chaps. III.A, III.D, and III.E and the related Apps. A through F and App. I: S. Kleinheins; Chap. IV and the related App. G: D. Wilhelm; App. H: G. Henneges; compilation of the material: G. Henneges and S. Kleinheins; and editor of this volume: G. Henneges. 


\section{REFERENCES}

1. E. A. Fischer, KfK, personal communication, January 14, 1986.

2. N. G. Cooper, "An Invitation to Participate in the LASL Equation of State Library," Los Alamos Scientific Laboratory brochure LA-79-6 (February 1980).

3. F. Dowell, "SESAME '83: Report on the Los Alamos Equation of State Library," Los Alamos National Laboratory report LALP-83-4 (February 1983).

4. B. I. Bennett, J. D. Johnson, G. I. Kerley, and G. T. Rood, "Recent Development in the SESAME Equation of State Library," Los Alamos Scientific Laboratory report LA-7130 (February 1978).

5. K. S. Holian (Editor), "T-4 Handbook of Material Properties Data Bases, Vol. Ic: Equations of State," Los Alamos Scientific Laboratory report LA-10160-MS (November 1984).

6. J. Abdallah Jr., G. I. Kerley, B. I. Bennett, J. D. Johnson, R. C. Alberts, and W. F. Huebner, "HYDSES: A Subroutine Package for Using SESAME in Hydrodynamics Codes," Los Alamos Scientific Laboratory report LA-8209 (June 1980).

7. Charles W. Cranfill, "EOSPAC: A Subroutine Package for Accessing the Los Alamos Sesame EOS Data Library," Los Alamos National Laboratory report LA-9728-M (August 1983).

8. J. Abdallah, "User's Manual for GRIZZLY," Los Alamos National Laboratory report LA-10244-M (September 1984).

9. J. F. Barnes and G. P. Devault, "Equations of State for LMFBR Materials," Proc. Int. Meeting on Fast Reactor Safety and Related Physics (Chicago, Illinois, October 5-8, 1976).

10. J. F. Barnes, "An Equation of State for Sodium Over an Extended Temperature and Density Range," IAEA Symposium on Thermodynamics of Nuclear Materials (Vienna, October 21-25, 1974).

11. U. Seydel, and W. Fucke, "Experimental Determination of Critical Data of Liquid Molybdenum," J. Phys. F 8, L157 (1978).

12. E. A. Fischer, "Equation of State for Solid Fuel for Use in the AFDM Code," internal KfK report (1988).

12. J. K. Fink, M. G. Chasanov, and L. Leibowitz, "Properties for Reactor Safety Analysis," Argonne National Laboratory report ANL-CEN-RSD-82-2 (1982). 
13. P. Nikopoulos and B. Schulz, "Density, Thermal Expansion of Stainless Steel, and Interfacial Properties of $\mathrm{UO}_{2}$-Stainless Steel Above $1690 \mathrm{~K}$," J. Nucl. Mater. 82 (1979).

14. P. Nikopoulos, E. Nold, H. Schneider, and B. Schultz, "The Influence of Sodium Vapor on the Wetting of $\mathrm{UO}_{2}$-Stainless Steel 1.4970 and Its Interpretation Using Scanning Auger Spectroscopy," J. Nucl. Mat. 101, 277 (1981).

15. P. Nikopoulos, S. Nazare, and F. Thümmler, "Surface, Grain Boundary, and Interfacial Energies in $\mathrm{UO}_{2}$ and $\mathrm{UO}_{2}-\mathrm{Ni}$," J. Nucl. Mat. 71, 89 (1977).

16. H. Matzke and T. Inone, "The Surface Energy of $\mathrm{UO}_{2}$ as Determined by Hertzian Indention," J. Nucl. Mat. 91, 205 (1980).

17. J. J. Bikermann, Surface Chemistry, Academic Press, New York (1958).

18. W. R. Bohl and L. B. Luck, "SIMMER-II: A Computer Program for LMFBR Disrupted Core Analysis," Los Alamos National Laboratory report LA-11415-MS (June 1990).

19. T. C. Chawla, et. al., "Thermophysical Properties of Mixed Oxide Fuel and Stainless Steel Type 316 for Use in Transition Phase Analysis," Nucl. Eng. and Design, Vol. 67, pp. 57-74 (1981).

20. R. B. Bird, W. E. Steward, and E. N. Lightfoot, "Transport Phenomena," Dept. of Chem. Eng., University of Wisconsin, John Wiley, New York (1976).

21. R. C. Reid, J. M. Prausnitz, and T. K. Sherwood, "The Properties of Gases and Liquids," McGraw-Hill, Third Ed., New York (1977).

22. L. Leibowitz, "Properties for LMFBR Safety Analysis," Argonne National Laboratory report ANL-CEN-RSD-76-1, Supplement 1 (April 1976).

23. L. L. Smith, "SIMMER-II: A Computer Program for LMFBR Disrupted Core Analysis," Los Alamos Scientific Laboratory report NUREG/CR-0453, LA-7515-M (June 1980).

24. S. Kleinheins, "SIMMER-II.9: Fluid Dynamics and Point Kinetics Program Structuring, Flowcharts, Tables, and Equations," internal KfK report (August 1984). 


\section{APPENDIX A NOTATION}

The notation used for the symbols and generic subscripts of the quantities in this volume of the AFDM documentation follows the common AFDM nomenclature in most respects. Differences and extensions occur only in the material and components subscript and cell subscript notation where the SIMMER-II documentation 23,24 usage has been followed.

Table A.I shows the material and component assignment. The material subscript $M$ runs from 1 to 3 ; it is separated from any preceding subscript by a comma. (Because the material properties in this volume subscripted with $\mathbf{M}$ are not used subscripted with $\mathrm{m}$ or $\mathrm{r}$-see below-the comma could have been omitted without risking confusion.) The density-component subscript $m$ runs from 1 to 7 ; the energy-component subscript $r$ runs from 1 to 5 . Both subscripts are appended without comma to any preceding subscript. The density-component subscripts are used with densities $\rho$ and macrodensities $\bar{\rho}$, and the energy-component subscripts are used with volume fractions $\alpha$ and temperatures T. Pressures $p$, specific internal energies $e$, and specific enthalpies $i$ can have both kinds of subscripts. Thus, this notation is not unique for the vapor components (e.g., p5 could be the partial pressure of vapor 1 or the total vapor pressure). To avoid the ambiguity, the energycomponent subscript $G$ is used instead of 5 wherever there is the danger of confusion with the density component subscript 5 . The velocity component $\mathrm{q}$ can have the values $\mathrm{L} 1, \mathrm{~L} 2$, or $\mathrm{G}$.

The material subscript $M$ is intended for the SAEOS; only in special cases (see Sec. III.A.4) are quantities subscripted with $M$ used for the TEOS, as well. Generally, for the TEOS, the material index $M^{\prime}$ is used instead, which has no rigid assignment to the component.

When needed by the descriptions in this volume, we use the default assignment of Table A.I.

Material and component subscripts can be functions of other subscripts; e.g., $M(m)$ indicates the material number $M$ of the density-component number $m$, etc.

Cell subscripts are used both to indicate that a quantity is an element of a cell vector array and to point to the position (relative to the center of the current cell) where the quantity is defined. They are attached to symbols and array names after any other subscripts. In this volume, the cell subscript notation of Fig. A.1 ${ }^{13}$ is 
TABLE A-I

AFDM MATERIAL AND COMPONENT ASSIGNMENT

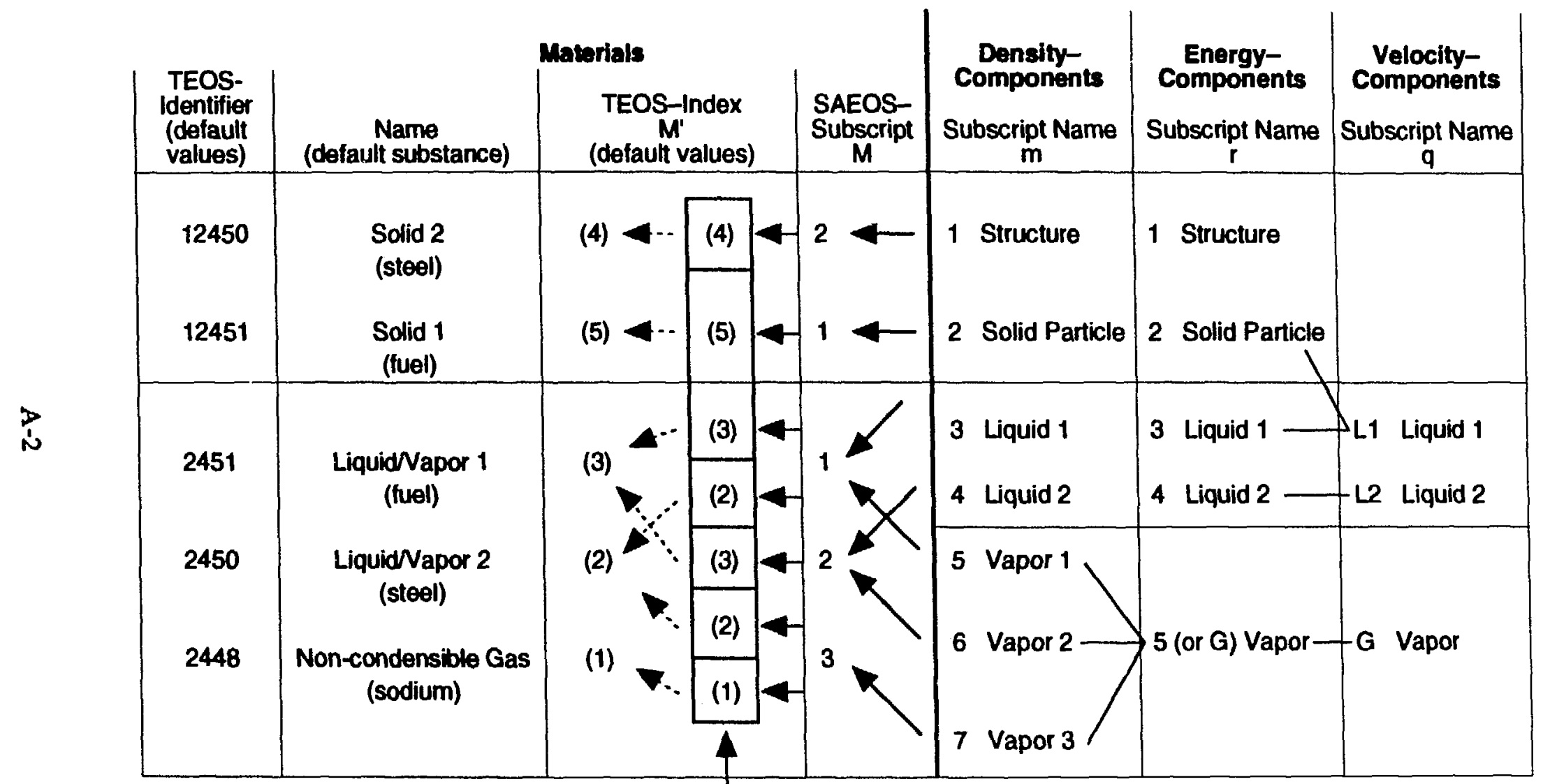

NRTAB Array (input) 


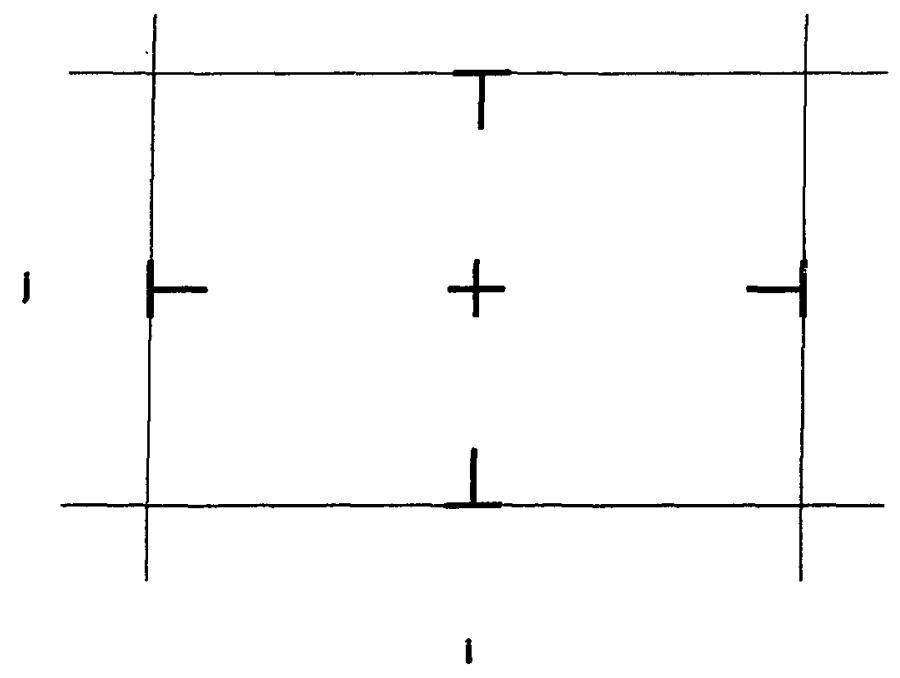

Fig. A.1.

Cell subscript notation.

applied, with the subscript + denoting the center of the current cell; it corresponds to the array subscript $i j$, which covers the range of the mesh cells involved.

The special notation used for the definition of the derivatives and for the routine specifications and flowcharts is defined in Chap. III.C, Apps. B and C, respectively. 


\section{APPENDIX B \\ ROUTINE SPECIFICATIONS}

In this chapter, the specifications of the following routines are compiled:

EOSUP

EOSAT

EOSPEQ

EOS2

EOSTAT

EOSETS

EOST

EOSPHC

EOSTP

EOSD2

EOSVD1

EOSS1

EOSPD

SESINI

SPNINI

SESPRT

SESEOS

VSTOP

VSPOT

VSELOT

VSTOEL

SPINDM

LAGRAN

URANUS

THEPHY 
The order of the routine specifications is that of the routine treatment in Chaps. III.C, III.D, and IV.

The specifications follow a common format. In the upper-right-hand corner, the option is indicated under which the routine is inserted into the compilable code; "SESAME off/on" or "KQQM1 on/KQQM2 on" means that two versions of the routine exist, one for each option. In that case, the argument list may differ (not always for good reasons) between the two options as stated by the "if" clauses and footnotes.

The first column of the argument list contains the dummy variable and array names of the routine, the latter with the dimensions as used in the code; the dimensions declared in the DIMENSION statements are listed under "Remarks."

Common variables and arrays (e.g., MIJBP2) are not commented on in this chapter; refer to Vol. VII, App. G, of the AFDM documentation for a description. 
Purpose: For the SESAME option "on", EOSUP calls the routines to load the EOS tables into the storage arrays and to generate spinodal data (if requested by input). EOSUP also extracts quantities from the saturation and sublimation tables, e.g.,

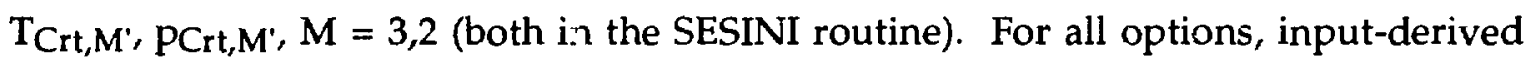
quantities such as esol, $1, \mathrm{e}_{\mathrm{Liq}, 1}, \mathrm{~h}_{\mathrm{f}, \mathrm{l}}, T_{r}^{0}, e_{r}^{0}, \mathrm{r}=1, \ldots, 4$, and $\Delta \mathrm{T}_{\mathrm{h}}=\mid \mathrm{T}_{\mathrm{Sat}, 1}\left\{10^{5} \mathrm{~Pa}\right\}$ $\mathrm{T}_{\mathrm{Sat}, 2}\left\{10^{5} \mathrm{~Pa}\right\} \mid$ are calculated (for the SESAME option "on," replace the above $\mathrm{M}$ subscripts by the corresponding $\mathrm{M}^{\prime}$ subscripts).

Arguments: None

Called Routines: If the SESAME option is "on": SESINI, SPNINI, VSTOP, VSELOT. 
Purpose: Given the vectors of the component input temperatures $T_{r+}^{i} r=1, \ldots, 5$, of the component input volume fractions $\alpha_{\mathrm{r} t^{\prime}}^{\mathrm{i}} \mathrm{r}=1, \ldots, 5$, and (for single-phase cells) of the input cell pressure $p_{+}^{i}$, EOSAT obtains (among other quantities) the vectors of the component densities $\rho_{\mathrm{m}+}, \mathrm{m}=1, \ldots, 7$, of the component specific-internal energies $\mathrm{e}_{\mathrm{r}+}$, $r=1, \ldots, 5$, (for two-phase cells) of the cell pressure $p_{+}$, and of the following quantities, under the assumption of thermodynamic equilibrium:

vapor component saturation temperature $\mathrm{T}_{\mathrm{Sat}, \mathrm{M}(\mathrm{m})+,} \mathrm{m}=5,6$ :

1. TSat, 1+

2. TSat, $2+$

(For the SESAME option "on" replace the above M subscripts by the corresponding M' subscripts).

\section{Arguments:}

NC - input : total number of cells in the vectors.

TI(NC,5) - input: component temperatures $T_{r+}^{i}$.

AI(NC,5) - input: component volume fractions $\alpha_{\mathrm{r}+}^{\mathrm{i}}$.

PC(NC) - input: single-phase cell pressure $p_{\text {cell }}^{i}$.

PG3(NC) - input: noncondensible gas pressure $p_{7_{+}}^{i}$

(If SESAME option"off," next argument:)

ER(NC,6) - auxiliary array.

(If SESAME option "on," next argument:)

ER(NC,43) - auxiliary array.

$\mathrm{AO}(\mathrm{NC}, 5)$ - output: component volume fraction $\alpha_{\mathbf{r}+}$.

RBO(NC,7) - output: component macrodensities $\bar{\rho}_{\mathrm{m}+}$.

$\mathrm{RO}(\mathrm{NC}, 7)$ - output: component densities $\rho_{\mathrm{m}+}$.

$\mathrm{EO}(\mathrm{NC}, 5)$ - output: component specific-internal energies $e_{r+}$.

$\mathrm{PO}(\mathrm{NC}) \quad$ - output: cell pressure $\mathrm{p}_{+}$.

TSO(NC,2) - output: vapor component saturation temperature; see above.

ERR - output: error code.

Remarks: The dimension of the array is (MIJBP2) or (MIJBP2,*), respectively.

Called Routines: If the SESAME option is "on": SESEOS, VSTOP, VSPOT, VSELOT. 
Purpose: Given the vectors of the component macrodensities $\bar{\rho}_{\mathrm{m}+} \mathrm{m}=1, \ldots, 7$, and of the component temperatures $T_{r+}, r=1, \ldots, 5$, EOSPEQ obtains (among other quantities) the vectors of the component pressures $\mathrm{Pr}_{r}, \mathrm{r}=1, \ldots, 4, \mathrm{G}$, and of the component volume fractions $\alpha_{r+,} r=1, \ldots, 5$, under the assumption of mechanical equilibrium.

\section{Arguments:}

NC - input: total number of cells in the vectors.

$\mathrm{RB}(\mathrm{NC}, 7) \quad$ - input: component macrodensities $\bar{\rho}_{\mathrm{m}+}$.

$\mathrm{T}(\mathrm{NC}, 5) \quad$ - input: component temperatures $\mathrm{T}_{\mathrm{r}+\text {. }}$

$\operatorname{ALP}(\mathrm{NC}, 5) \quad$ - output: component volume fractions $\alpha_{\mathrm{r}+}$.

$\mathrm{RHO}(\mathrm{NC}, 7) \quad$ - input: initial guess for component densities $\rho_{\mathrm{m}+i}$ output: component densities $\rho_{\mathbf{m}+}$.

SIE(NC,7) - output: component specific-internal energies $e_{m+}$.

PM(NC,5) - output: (energy-) component pressures $p_{r+}, r=1, \ldots, 4, g$.

$\mathrm{PG}(\mathrm{NC}, 3) \quad$ - output: (density-) component pressures $\mathrm{p}_{\mathrm{m}+}, \mathrm{m}=5,6,7$.

$\operatorname{DPDR}(\mathrm{NC}, 7) \quad$ - output: $\partial_{\rho} \mathrm{p}_{\mathbf{m}+}$.

$\operatorname{DPDT}(\mathrm{NC}, 7) \quad$ - output: $\partial_{\mathrm{T}} \mathrm{p}_{\mathrm{m}+}$.

$\operatorname{DEDR}(\mathrm{NC}, 7) \quad$ - output: $\partial_{\rho} \mathrm{e}_{\mathrm{m}+}$.

DEDT(NC,7) - output: $\partial_{\mathrm{T}} \mathrm{e}_{\mathrm{m}+}$.

NTP - output: total number of two-phase cells.

TTP(NC) - output: subscripts ij of the two-phase cells.

NSP - output: total number of single-phase cells.

TSP(NC) - output: subscripts ij of the single-phase cells.

$\operatorname{ER}(\mathrm{NC}, 71)$ - auxiliary array.

$\operatorname{LER}(\mathrm{NC}, 6)$ - auxiliary array.

IER(NC,2) - auxiliary array.

Remarks: The dimension of all arrays in (MIJBP2,*).

Called Routines: SESEOS, VSELOT, SPINDM, and some auxiliary routines. 
Purpose: Given the vectors of the component macrodensities $\bar{\rho}_{\mathrm{m} t^{\prime}} \mathrm{m}=1, \ldots, 7$, and of the component temperature $T_{r+}, r=1, \ldots, 5$, EOS2 obtains (among other quantities) the vectors of the pressure $\mathrm{pG}_{+}$, of the component volume fractions $\alpha_{r+}, r=1, \ldots, 5$, and of the component specific-internal energies $e_{m+}, m=1, \ldots, 7$ (if the SESAME option is "off": from input constants or straightforwardly calculated; if the SESAME option is "on": under the assumption of mechanical equilibrium):

\section{Arguments:}

NC - input: total number of cells in the vectors."

$\mathrm{T}(\mathrm{NC}, 5) \quad$ - input: component temperatures $\mathrm{T}_{\mathrm{r}+}$.

$\operatorname{RB}(\mathrm{NC}, 7)$ - input: component macrodensities $\bar{\rho}_{\mathrm{m}+}$.

$\operatorname{ALP}(N C, 5)$ - output: component volume fractions $\alpha_{r+}$.

SIE(NC,7) - output: component specific-internal energies $\mathbf{e}_{\mathbf{m}+}$.

RHO(NC,7) - input: initial guess for component densities $\rho_{m+}$. output: component densities $\rho_{\mathrm{m}+}$.

$\mathrm{P}(\mathrm{NC}) \quad$ output: vapor mixture pressure $\mathrm{PG}+$.

(If SESAME option "off," next argument:)

ER(NC,4) - auxiliary array.

(If SESAME option "on," next three arguments:)

ER(NC,114) - auxiliary array.

$\operatorname{IER}(N C, 4)$ - auxiliary array.

LER(NC,6) - auxiliary array.

Remarks: The dimension of all arrays is (MIJBP2, ${ }^{*}$ ).

Called Routines: If the SESAME option is "on": EOSPEQ.

"Instead of NC, IB and JB are used for SESAME "off." 
Purpose: Given the vectors of the component macrodensities $\overline{\boldsymbol{\rho}}_{\mathrm{m}+^{\prime}} \mathrm{m}=1, \ldots, 7$, and of the component temperature $T_{r+}, r=1, \ldots, 5$, EOSTAT obtains (among other quantities) the vectors of the pressure $\mathrm{PG}_{+}$, of the component volume fractions $\alpha_{r+}, r=1, \ldots, 5$, of the component specific-internal energies $e_{r+}, r=1, \ldots, 4, G$, and of the following quantities (if the SESAME option is "off": from input constants or straightforwardly calculated; if the SESAME option is "on": under the assumption of mechanical equilibrium):

vapor component saturation temperatures $\mathrm{T}_{\mathrm{Sat}, \mathrm{M}(\mathrm{m})+,} \mathrm{m}=5,6$ :

1. $\mathrm{T}_{\text {Sat, } 1+}$

2. $\mathrm{T}_{\text {Sat }, 2+}$

(For the SESAME option "on," replace the above M subscripts by the corresponding M' subscripts.)

\section{Arguments:}

NC - input: total number of cells in the vectors."

$\mathrm{T}(\mathrm{NC}, 5) \quad$ - input: component temperatures $\mathrm{T}_{\mathrm{r}+}$.

TS(NC,2) - output: vapor component saturation temperatures; see above.

$\mathrm{RB}(\mathrm{NC}, 7)$ - input: component macrodensities $\overline{\boldsymbol{\rho}}_{\mathrm{m}+}$.

$\operatorname{ALP}(\mathrm{NC}, 5)$ - output: component volume fractions $\alpha_{\mathrm{r}+}$.

SIE(NC,5) - output: component specific-internal energies; $e_{r+}$.

RHO(NC,7) - input: initial guess for component densities $\rho_{\mathrm{m}+}$. output: component densities $\rho_{\mathrm{m}+}$.

$P(N C) \quad$ - output: vapor mixture pressure $P_{G+}$.

(If SESAME option "off," next argument:)

ER(NC,5) - auxiliary array.

(If SESAME option "on," next three arguments:)

ER(NC,114) - auxiliary array.

LER(NC,6) - auxiliary array.

IER(NC,4) - auxiliary array.**

\footnotetext{
"Instead of NC, JB, JB, and JJB are used for SESAME "off."

"t Though not used, IER appears also for SESAME "off."
} 
Remarks: The dimension of all arrays is (MIJBP2,*).

Called Routines: If the SESAME option is "on": EOSPEQ, VSTOP. 
Purpose: Given the vectors of the component macrodensities $\bar{\rho}_{\mathrm{m}+^{\prime}} \mathrm{m}=1, \ldots, 7$, and of the component temperature $T_{r+}, r=1, \ldots, 5$, EOSETS obtains (among other quantities) the vectors of the component volume fractions $\alpha_{r+}, r=1, \ldots, 5$, and of the component specific-internal energies $e_{r+}, r=1, \ldots, 4, G$ (if the SESAME option is "off": from input constants or straightforwardly calculated; if the SESAME option is "on": under the assumption of mechanical equilibrium):

\section{Arguments:}

NC - input: total number of cells in the vectors.

$\mathrm{T}(\mathrm{NC}, 5) \quad$ - input: component temperatures $\mathrm{T}_{\mathrm{r}+}$.

$R B(N C, 7)$ - input: component macrodensities $\bar{\rho}_{\mathrm{m}+}$.

(If SESAME option "on," next argument:)

RHO(NC,7) - input: initial guess for component densities $\rho_{m+}$. output: component densities $\rho_{\mathrm{m}+}$.

SIE(NC,5) - output: component specific-internal energies $e_{r+}$. (If SESAME option "on," next three arguments:)

ER(NC,119) - auxiliary array.

IER(NC,4) - auxiliary array.

LER(NC,6) - auxiliary array.

Remarks: The dimension of all arrays is (MIJBP2, $\left.{ }^{*}\right)$.

Called Routines: IF the SESAME option is "on": EOSPEQ. 
Purpose: Given the vectors of the component macrodensities $\bar{\rho}_{\mathrm{m}+^{\prime}} \mathrm{m}=1, \ldots, 7$, and of the component specific-internal energies $e_{r+}, r=1, \ldots, 4, G$, EOST obtains (among other quantities) the vectors of the densities $\rho_{\mathrm{m}+}, \mathrm{m}=1, \ldots, 7$, of the component temperature $T_{r+}, r=1, \ldots, 5$, and of the following quantities (if the SESAME option is "off": from input constants or straightforwardly calculated; if the SESAME option is "on": under the assumption of mechanical equilibrium):

vapor component saturation temperatures $\mathrm{T}_{\mathrm{Sat}, \mathrm{M}(\mathrm{m})+}, \mathrm{m}=5,6$ :

1. $\mathrm{T}_{\text {Sat, } 1+}$

2. $\mathrm{T}_{\text {Sat }, 2+}$

(For the SESAME option "on," replace the above M subscripts by the corresponding M' subscripts).

\section{Arguments:}

NC - input: total number of cells in the vectors.

$\mathrm{T}(\mathrm{NC}, 5) \quad$ - input: initial guess for component temperatures $\mathrm{T}_{\mathrm{r}+}$; output: component temperatures $\mathrm{T}_{\mathrm{r}+}$.

TS(NC,2) - output: vapor component saturation temperatures; see above.

RB(NC,7) - input: component macrodensities $\bar{\rho}_{\mathrm{m}+}$.

ALP(NC,5) - output: component volume fractions $\alpha_{r+}$.

SIE(NC,5) - output: component specific-internal energies $e_{r+}$.

RHO(NC,7) - input: component densities $\rho_{m+}$.

(If SESAME option "off," next two arguments:)

ER(NC,19) - auxiliary array.

$\operatorname{IER}(\mathrm{NC}, 21)$ - auxiliary array.

(If SESAME option "on," next three arguments:)

$\mathrm{ER}(\mathrm{NC}, 114)$ - auxiliary array.

$\operatorname{IER}(\mathrm{NC}, 2)$ - auxiliary array.

LER(NC,6) - auxiliary array.

Remarks: The dimension of all arrays is (MIJBP2, ${ }^{*}$ ).

"Instead of NC, IB and JB are used for SESAME "off." 
Called Routines: If the SESAME option is "on": SESEOS, VSTOP, VSELOT, VSTOEL, SPINDM, and some auxiliary routines. 
Purpose: Given the vectors of the component macrodensities $\bar{\rho}_{\mathrm{m}+^{\prime}} \mathrm{m}=1, \ldots, 7$, and of the component temperatures $T_{r+} r=1, \ldots, 5$, EOSPHC obtains the vectors of the following quantities (if the SESAME option is "off": from input constants or straightforwardly calculated; if the SESAME option is "on": under the assumption of mechanical equilibrium):

liquid component saturation pressures $\mathrm{PSat}, \mathrm{M}(\mathrm{m})+, \mathrm{m}=3,4$ :

1. PSat, $1+$

2. PSat, $2+$

vapor component saturation temperatures $T_{S a t, M(m)+}, M=5,6$ :

1. TSat, $1+$

2. TSat, $2+$

vapor component specific vaporization enthalpies $h_{\mathrm{lg}, M(m)}+, m=5,6$ :

1. $\mathrm{h}_{\mathrm{g}, 1+}=\mathrm{i}_{\mathrm{Vap}, 1+}-\mathrm{i}_{\mathrm{C}}, 1+$

2. $\mathrm{h}_{\mathrm{lg}, 2+}=\mathrm{i}_{\mathrm{Vap}, 2+}-\mathrm{i}_{\mathrm{Con}, 2+}$

(For the SESAME option "on," replace the above M subscripts by the corresponding M' subscripts).

Solid component, liquid component, and vapor mixture specific heat capacities:
1. $\left[\frac{\mathrm{de}_{1}}{\mathrm{dT}}\right]_{1}$
2. $\left[\frac{\mathrm{de}_{2}}{\mathrm{dT}_{2}}\right]_{+}$
3. $\left[\frac{\mathrm{de}_{3}}{\mathrm{dT}_{3}}\right]_{+}$
4. $\left[\frac{\mathrm{de}_{4}}{\mathrm{dT}_{4}}\right]$
5. $\left[\frac{\mathrm{de}_{\mathrm{G}}}{\mathrm{dT}_{5}}\right]_{+}$ 
Vapor component specific heat capacities:
1. $\left[\frac{\mathrm{de}_{5}}{\mathrm{dT}_{5}}\right]_{+}$
2. $\left[\frac{\mathrm{de}_{6}}{\mathrm{dT}_{5}}\right]_{+}$
3. $\left[\frac{\mathrm{de}_{7}}{\mathrm{dT}_{5}}\right]_{+}$

\section{Arguments:}

NC - input: total number of cells in the vectors.

$\mathrm{RB}(\mathrm{NC}, 7) \quad$ - input: component macrodensities $\bar{\rho}_{\mathrm{m}+}$.

$\mathrm{T}(\mathrm{NC}, 5) \quad$ - input: component temperatures $\mathrm{T}_{\mathrm{r}+}$.

(If SESAME option "on," next argument:)

$\mathrm{RHO}(\mathrm{NC}, 7)$ - input: initial guess for component densities $\rho_{\mathrm{m}+\text {; }}$ output: component densities $\rho_{\mathbf{m}+\text {. }}$.

PS(NC,2) - output: liquid component saturation pressures; see above.

TS(NC,2) - output: vapor component saturation temperatures; see above.

$\mathrm{HV}(\mathrm{NC}, 2) \quad$ - output: vapor component-specific vaporization enthalpies; see above.

$\mathrm{HCV}(\mathrm{NC}, 5)$ - output: solid component, liquid component, and vapor mixture specific heat capacities, see above.

HCCV(NC,3) - output: vapor component specific heat capacities, see above.

(If SESAME option "on," next three arguments:)

$\mathrm{ER}(\mathrm{NC}, 119)$ - auxiliary array.

IER(NC,4) - auxiliary array.

LER(NC,10) - auxiliary array.

Remarks: The dimension of all arrays is (MIJBP2,*).

Called Routines: If the SESAME option is "on": EOSPEQ, SESEOS, VSTOP, VSPOT, and some auxiliary routines. 
Purpose: Called for two-phase cells only. Given the vectors of the component macrodensities $\bar{\rho}_{\mathrm{m}+}, \mathrm{m}=1, \ldots, 7$, and of the component temperature $\mathrm{T}_{\mathrm{r}+}, \mathrm{r}=1, \ldots, 5$, EOSTP obtains the vectors of the component volume fractions $\alpha_{r+}, r=1, \ldots, 5$, and of the following quantities (if the SESAME option is "off": from input constants or straightforwardly calculated; if the SESAME option is "on": under the assumption of mechanical equilibrium):

vapor component saturation temperatures $\mathrm{T}_{\mathrm{Sat}, \mathrm{M}(\mathrm{m})+}, \mathrm{M}=5,6$ :

1. TSat, $1+$

2. $\mathrm{T}_{\mathrm{Sat}, 2+}$

Various quantities, such as vapor component specific-condensate enthalpies $\mathrm{i}_{\text {Con, } M(m)+} \mathrm{m}=5,6$, component specific-internal energies $\mathrm{e}_{\mathrm{m}+}, \mathrm{m}=3,4, \mathrm{G}$, vapor component-specific enthalpies $i_{m+}, m=5,6,7$, and vapor component-specific vaporization enthalpies $h_{l g, M(m)+} m=5,6$ :

1. $\mathrm{i}_{\mathrm{Con}, 1+}$

2. $\mathrm{i}_{\mathrm{Con}, 2+}$

3. $\mathrm{e}_{3+}$

4. $\mathrm{e}_{4+}$

5. $\mathrm{i}_{5+}$

6. $i_{6+}$

7. $i_{7+}$

8. $\mathrm{e}+$

9. $\mathrm{h}_{\mathrm{gg}, 1+}=\mathrm{i}_{\text {Vap }, 1+}-\mathrm{i} \operatorname{Con}, 1+$

10. $h_{\mathrm{lg}, 2+}=\mathrm{i}_{\text {Vap }, 2+}-\mathrm{i}_{\mathrm{Con}, 2+}$

Vapor component-specific condensate enthalpy derivatives:
1. $\left[\frac{\mathrm{di}_{\text {Con }, 1}}{\mathrm{~d} \bar{\rho}_{3}}\right]_{+}\left[\frac{\mathrm{di}_{\text {Con.2. }}}{\mathrm{d} \bar{\rho}_{3}}\right]_{+}$
2. $\left[\frac{\mathrm{di}_{\text {Con, }, 1}}{\mathrm{~d} \bar{\rho}_{4}}\right]_{+}\left[\frac{\mathrm{di}_{\text {Con, } 2}}{\mathrm{d \overline { \rho } _ { 4 }}}\right]_{+}$ 

3. $\left[\frac{\mathrm{di}_{\text {Con,1 }}}{\mathrm{dT}_{3}}\right]_{+}\left[\frac{\mathrm{di}_{\text {Con, } 2}}{\mathrm{dT}_{3}}\right]_{+}$
4. $\left[\frac{\mathrm{di}_{\text {Con.1 }}}{\mathrm{dT}_{4}}\right]_{+}\left[\frac{\mathrm{di}_{\text {Con.2. }}}{\mathrm{dT}_{4}}\right]_{+}$
5. $\left[\frac{\mathrm{di}_{\text {Con,1 }}}{\mathrm{dT}_{5}}\right]_{+}\left[\frac{\mathrm{di}_{\text {Con, } 2}}{\mathrm{dT}_{5}}\right]_{t}$
6. $\left[\frac{\mathrm{di}_{\text {Con,1 }}}{\mathrm{d} \bar{\rho}_{5}}\right]_{+}\left[\frac{\mathrm{di}_{\text {Con,2 }}}{\mathrm{d} \bar{\rho}_{5}}\right]_{+}$
7. $\left[\frac{\mathrm{di}_{\text {Con,1 }}}{\mathrm{d} \vec{\rho}_{6}}\right]_{+}\left[\frac{\mathrm{di}_{\text {Con,2 }}}{\mathrm{d} \bar{\rho}_{6}}\right]_{t}$

Vapor component specific vaporization enthalpy derivatives:
1. $\left[\frac{\mathrm{dh}_{\mathrm{lg}_{1}, 1}}{\mathrm{~d} \bar{\rho}_{3}}\right]_{+}\left[\frac{\mathrm{dh} \mathrm{lg}, 2_{2}}{\mathrm{~d} \bar{\rho}_{3}}\right]_{+}$
$2\left[\frac{\mathrm{dh}_{\mathrm{g}, 1}}{\mathrm{~d} \bar{\rho}_{4}}\right]_{+}\left[\frac{\mathrm{dh} \mathrm{1g}, 2_{2}}{\mathrm{~d} \bar{\rho}_{4}}\right]_{+}$
$3\left[\frac{\mathrm{dh}_{\mathrm{g}_{\mathrm{g}},}}{\mathrm{dT}_{3}}\right]_{+}\left[\frac{\mathrm{dh}_{\mathrm{g} .2}}{\mathrm{dT}_{3}}\right]_{+}$
4. $\left[\frac{\mathrm{dh}_{\mathrm{g}, 1}}{\mathrm{dT}_{4}}\right]_{+}\left[\frac{\mathrm{dh}_{\mathrm{g}, 2}}{\mathrm{dT}_{4}}\right]_{+}$
5. $\left[\frac{\mathrm{dh}_{\mathrm{g}, 1}}{\mathrm{dT}_{5}}\right]_{+}\left[\frac{\mathrm{dh}_{\mathrm{g}_{2}, 2}}{\mathrm{dT}_{5}}\right]_{+}$
6. $\left[\frac{d h_{g, 1}}{d \bar{\rho}_{5}}\right]_{+}\left[\frac{d h_{g, 2}}{d \bar{\rho}_{5}}\right]_{+}$
7. $\left[\frac{\mathrm{dh}_{\mathrm{lg}_{1}, 1}}{\mathrm{~d} \bar{\rho}_{6}}\right]_{+}\left[\frac{\mathrm{dh}_{\mathrm{g}, 2}}{\mathrm{~d} \bar{\rho}_{6}}\right]_{+}$

Vapor component specific enthalpy derivatives:
1. $\left[\frac{\mathrm{di}_{5}}{\mathrm{~d} \bar{\rho}_{3}}\right]_{+}\left[\frac{\mathrm{di}_{6}}{\mathrm{~d} \bar{\rho}_{3}}\right]_{+}$
2. $\left[\frac{\mathrm{di}_{5}}{\mathrm{~d} \bar{\rho}_{4}}\right]_{+}\left[\frac{\mathrm{di}_{6}}{\mathrm{~d} \bar{\rho}_{4}}\right]_{+}$ 

3. $\left[\frac{\mathrm{di}_{5}}{\mathrm{dT}_{3}}\right]_{+}\left[\frac{\mathrm{di}_{6}}{\mathrm{dT}_{3}}\right]_{+}$
4. $\left[\frac{\mathrm{di}_{5}}{\mathrm{dT}_{4}}\right]_{4}\left[\frac{\mathrm{di}_{6}}{\mathrm{dT}_{4}}\right]_{4}$
5. $\left[\frac{\mathrm{di}_{5}}{\mathrm{dT}_{5}}\right]_{+}\left[\frac{\mathrm{di}_{6}}{\mathrm{dT}_{5}}\right]_{+}$
6. $\left[\frac{\mathrm{di}_{5}}{\mathrm{~d} \bar{\rho}_{5}}\right]_{4}\left[\frac{\mathrm{di}_{6}}{\mathrm{~d} \bar{\rho}_{5}}\right]_{t}$
7. $\left[\frac{d i_{5}}{d \bar{\rho}_{6}}\right]_{+}\left[\frac{d i_{6}}{d \bar{\rho}_{6}}\right]_{+}$

Vapor mixture-specific-internal energy derivatives:
1. $\left[\frac{d e_{G}}{d \bar{\rho}_{3}}\right]$
2. $\left[\frac{d e_{G}}{d \bar{\rho}_{4}}\right]_{+}$
3. $\left[\frac{d e_{G}}{d T_{3}}\right]_{4}$
4. $\left[\frac{\mathrm{de}_{\mathrm{G}}}{\mathrm{dT}}\right]_{4}$
5. $\left[\frac{d_{G}}{d T_{5}}\right]_{+}$
6. $\left[\frac{d e_{G}}{d \bar{\rho}_{5}}\right]_{+}$
7. $\left[\frac{d e_{G}}{d \bar{\rho}_{6}}\right]_{4}$

Vapor component saturation temperature derivatives:
1. $\left[\frac{d T_{S_{a t, 1}}}{d \bar{\rho}_{3}}\right]_{+}\left[\frac{d T_{\text {Sat, } 2}}{d \bar{\rho}_{3}}\right]_{+}$
2. $\left[\frac{d T_{S_{a t, 1}}}{d \bar{\rho}_{4}}\right]_{+}\left[\frac{d T_{S_{\text {Sat }, 2}}}{d \bar{\rho}_{4}}\right]_{+}$ 

3. $\left[\frac{\mathrm{dT}_{\mathrm{Sat}_{1},}}{\mathrm{dT}_{3}}\right]_{+}\left[\frac{\mathrm{dT}_{\mathrm{Sal}_{2}}}{\mathrm{dT}_{3}}\right]_{+}$
4. $\left[\frac{\mathrm{dT}_{S_{a t}, 1}}{\mathrm{dT}_{4}}\right]_{+}\left[\frac{\mathrm{dT}_{\mathrm{Sat}, 2}}{\mathrm{dT}_{4}}\right]_{+}$
5. $\left[\frac{\mathrm{dT}_{\mathrm{Sat}_{1} 1}}{\mathrm{dT}_{5}}\right]_{+}\left[\frac{\mathrm{dT}_{\mathrm{Sat}_{2}}}{\mathrm{dT}_{5}}\right]_{+}$
6. $\left[\frac{\mathrm{dT}_{\mathrm{Sat}, 1_{1}}}{\mathrm{~d} \bar{\rho}_{5}}\right]_{+} \quad\left[\frac{\mathrm{dT}_{\text {Sat }, 2}}{\mathrm{~d} \bar{\rho}_{5}}\right]_{+}$

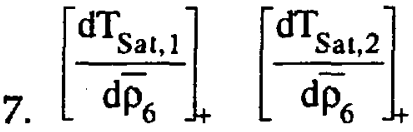

Liquid component specific-internal energy derivatives:
1. $\left[\frac{\mathrm{de}_{3}}{\mathrm{~d} \bar{\rho}_{3}}\right]_{+}\left[\frac{\mathrm{de}_{4}}{\mathrm{~d} \bar{\rho}_{3}}\right]_{+}$
2. $\left[\frac{\mathrm{de}_{3}}{\mathrm{d \overline { \rho } _ { 4 }}}\right]_{+}\left[\frac{\mathrm{de}}{\mathrm{d} \bar{\rho}_{4}}\right]_{+}$
3. $\left[\frac{\mathrm{de}_{3}}{\mathrm{dT}_{3}}\right]_{+}\left[\frac{\mathrm{de}_{4}}{\mathrm{dT}_{3}}\right]_{+}$
4. $\left[\frac{\mathrm{de}_{3}}{\mathrm{dT}_{4}}\right]_{+}\left[\frac{\mathrm{de}_{4}}{\mathrm{dT}_{4}}\right]_{+}$
5. $\left[\frac{\mathrm{de}_{3}}{\mathrm{dT}_{5}}\right]_{+} \quad\left[\frac{\mathrm{de}_{4}}{\mathrm{dT}_{5}}\right]_{+}$
6. $\left[\frac{\mathrm{de}}{\mathrm{d} \bar{\rho}_{5}}\right]_{+}\left[\frac{\mathrm{de}_{4}}{\mathrm{de}_{5}}\right]_{+}$
7. $\left[\frac{\mathrm{de}_{3}}{\mathrm{~d} \bar{\rho}_{6}}\right]_{+}\left[\frac{\mathrm{de}_{4}}{\mathrm{~d} \bar{\rho}_{6}}\right]_{+}$

(For the SESAME option "on," replace the above M subscripts by the corresponding M' subscripts.)

\section{Arguments:}

NC

RE $\left.{ }^{\prime} \mathrm{NC}, 7\right)$

$\mathrm{T}(\mathrm{NC}, 5)$
- input: total number of cells in the vectors.

- input: component macrodensities $\bar{\rho}_{\mathrm{m}+}$.

- input: component temperatures $T_{r+}$. 
(If SESAME option "on," next two arguments:)
ALP(NC,5) - output: component volume fractions $\alpha_{\mathrm{r}+}$.
$\mathrm{RHO}(\mathrm{NC}, 7)$ - input: initial guess for component densities $\rho_{\mathrm{m}+\text {; }}$ output: component densities $\rho_{m+}$.
$\operatorname{TSAT}(\mathrm{NC}, 2) \quad$ - output: vapor component saturation temperatures; see above.
$\operatorname{EOSV}(\mathrm{NC}, 10)$ - output: various quantities; see above.
$\operatorname{DEC}(\mathrm{NC}, 7,2) \quad$ - output: vapor component specific-condensate enthalpy derivatives; see above.

DHLG $(N C, 7,2)$ - output: vapor component specific vaporization enthalpy derivatives; see above.

$\operatorname{DEGM}(\mathrm{NC}, 7,2)$ - output: vapor component specific enthalpy derivatives; see above.

$\mathrm{DEG}(\mathrm{NC}, 7) \quad$ - output: vapor mixture specific internal energy derivatives; see above.

$\mathrm{DTS}(\mathrm{NC}, 7,2) \quad$ - output: vapor component saturation temperature derivatives; see above.

$\operatorname{DELM}(\mathrm{NC}, 7,2)$ - output: liquid component specific-internal energy derivatives; see above.

(If SESAME option "off," next argument:)
$\mathrm{E}(\mathrm{NC}, 3)$
- auxiliary array.

(If SESAME option "on," next three arguments:)
$\mathrm{ER}(\mathrm{NC}, 114)$ - auxiliary array.
LER(NC,9) - auxiliary array.
$\operatorname{IER}(\mathrm{NC}, 4)$ - auxiliary array.

Remarks: The dimension of the arrays is $(\mathrm{MIJPP} 2, *)$ or $(\mathrm{MIJBP} 2,7, *)$, respectively.

Called Routines: If the SESAME option is "on": EOSPEQ, VSTOP, VSELOT, and some auxiliary routines. 
Purpose: Called for single-phase cells only. Given the vectors of the component macrodensities $\bar{\rho}_{\mathrm{m}+}, \mathrm{m}=1, \ldots, 7$, and of the component temperature $\mathrm{T}_{\mathrm{r}+,}, \mathrm{r}=1, \ldots, 5$, EOSD2 obtains the vectors of the component specific-internal energies $e_{r+}, r=1, \ldots, 4$, and of the following quantities (if the SESAME option is "off": from input constants or straightforwardly calculated; if the SESAME option is "on": under the assumption of mechanical equilibrium):

component specific heat capacities:
1. $\left[\frac{d e_{1}}{d T_{1}}\right]_{+}$
2. $\left[\frac{\mathrm{de}_{2}}{\mathrm{dT}}\right]_{+}$
3. $\left[\frac{\mathrm{de}_{3}}{\mathrm{dT}_{3}}\right]_{+}$
4. $\left[\frac{\mathrm{de}_{4}}{\mathrm{dT}}\right]_{+}$

\section{Arguments:}

NC - input: total number of cells in the vectors."

$\mathrm{RB}(\mathrm{NC}, 7)$ - input: component macrodensities $\bar{\rho}_{\mathrm{m}+}$.

$\mathrm{T}(\mathrm{NC}, 5) \quad$ - input: component temperatures $T_{r+}$.

(If SESAME option "on," next argument:)

$\mathrm{RHO}(\mathrm{NC}, 7)$ - input: initial guess for component densities $\rho_{\mathrm{m}+}$. output: component densities $\rho_{\mathrm{m}+}$.

DER(NC,4) - output: component specific heat capacities; see above.

SIE $(N C, 4) \quad$ - output: component specific-internal energies $e_{r+\cdot}{ }^{* *}$

(If SESAME option "on," next three arguments.

ER(NC,126) - auxiliary array.

IER(NC,4) - auxiliary array.

LER(NC,8) - auxiliary array. 
Remarks: The dimension of all arrays is (MIJBP2,*).

Called Routines: If the SESAME option is "on": EOSPEQ and some auxiliary routines. 
Purpose: Called for single-phase cells only. Given the vectors of the component macrodensities $\bar{\rho}_{\mathrm{m}+}, \mathrm{m}=1, \ldots, 7$, and of the component temperature $T_{r+}, r=1, \ldots, 5$, EOSVD1 obtains the vectors of the component volume fractions $\alpha_{\mathrm{r}+}, \mathrm{r}=1, \ldots, 5$, and of the following quantities (if the SESAME option is "off": from input constants or straightforwardly calculated; if the SESAME option is "on": under the assumption of mechanical equilibrium):

various quantities, such as component specific-internal energies $e_{m+}, m=1, \ldots, 6$, vapor mixture specific-internal energy $\mathrm{e}_{\mathrm{G}+}$, vapor component condensate and saturated vapor specific-internal energies e $\mathrm{Con}, \mathrm{M}(\mathrm{m})+$ and $\mathrm{e}_{\mathrm{Vap}} \mathrm{M}(\mathrm{m})+, \mathrm{m}=5,6$, respectively, vapor component saturation temperatures $T_{\text {Sat }, M(m)+}, m=5,6$, and

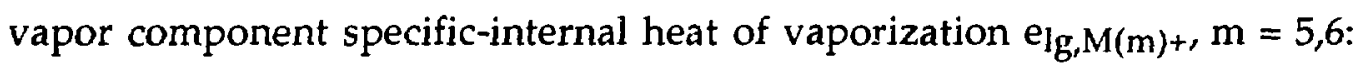

1. $e_{G+}$

2. TSat, $1+$

3. TSat,2+

4. eCon, $1+$

5. eCon,2+

6. eVap,1+

7. evap,2+

8. $\mathrm{e}_{3+}$

9. $\mathrm{e}_{4+}$

10. $\mathrm{e}_{\mathrm{g}, 1+}=\mathrm{eVap}, 1+=\mathrm{e}_{\mathrm{Con}, 1+}$

11. $e_{\mathrm{lg}, 2+}=\mathrm{e}_{\mathrm{Vap}, 2+}=\mathrm{e}_{\mathrm{Con}, 2+}$

12. $\mathrm{e}_{5+}$

13. $\mathrm{e}_{6+}$

14. $\mathrm{e}_{1+}$

15. $\mathrm{e}_{2+}$

Derivatives of various quantities:
1. $\left[\frac{\mathrm{de}_{\mathrm{G}}}{\mathrm{d} \bar{\rho}_{\mathrm{s}}}\right]_{+}$
2. $\left[\frac{d e_{G}}{d \bar{\rho}_{6}}\right]_{+}$ 
3. $\begin{aligned} & {\left[\frac{\mathrm{de}_{\mathrm{G}}}{\mathrm{d \textrm {T } _ { 5 }}}\right]_{+}} \\ & \text {4. }\left[\frac{\mathrm{de}}{\mathrm{d} \bar{p}_{5}}\right]_{+}\end{aligned}$

5. $\left[\frac{\mathrm{de}_{6}}{\mathrm{d \overline {p } _ { 6 }}}\right]_{+}$

6. $\left[\frac{\mathrm{de}_{5}}{\mathrm{dT}_{5}}\right]_{+}$

7. $\left[\frac{\mathrm{de}_{6}}{\mathrm{dT}_{5}}\right]_{+}$

8. $\left[\frac{\mathrm{de}_{3}}{\mathrm{~d} \overline{\mathrm{p}}_{3}}\right]_{+}$

9. $\left[\frac{d_{4}}{d \bar{p}_{3}}\right]_{+}$

10. $\left[\frac{\mathrm{de}_{3}}{\mathrm{~d} \overline{\mathrm{p}}_{4}}\right]_{+}$

11. $\left[\frac{\mathrm{de}_{4}}{\mathrm{~d} \bar{\rho}_{4}}\right]$.

12. $\left[\frac{\mathrm{de}_{3}}{\mathrm{dT}_{3}}\right]_{+}$

13. $\left[\frac{\mathrm{de}_{4}}{\mathrm{dT}_{3}}\right]_{+}$

14. $\left[\frac{\mathrm{de}_{3}}{\mathrm{dT}_{4}}\right]_{+}$

15. $\left[\frac{\mathrm{de}_{4}}{\mathrm{dT}_{4}}\right]$

16. $\left[\frac{\mathrm{de}_{\text {Con.1 }}}{\mathrm{d} \bar{\rho}_{5}}\right]$

17. $\left[\frac{\mathrm{de} \mathrm{Con}_{\text {Co. }}}{\mathrm{d} \bar{\rho}_{6}}\right]_{+}$

18. $\left[\frac{\mathrm{de}_{\text {Con.1 }}}{\mathrm{dT}_{5}}\right]_{+}$

19. $\left[\frac{\mathrm{de}_{\text {Con. }_{2}}}{\mathrm{dT}_{5}}\right]+$ 
20. $\left[\frac{d \rho_{g .1}}{d \bar{\rho}_{5}}\right]_{+}$

21. $\left[\frac{d j_{g .2}}{d \bar{\rho}_{6}}\right]_{+}$

22. $\left[\frac{d_{g_{g .1}}}{d T_{5}}\right]_{+}$

23. $\left[\frac{d_{1 g .2}}{d_{5}}\right]_{+}$

24. $\left[\frac{d \mathrm{v}_{\mathrm{va}, 1}}{\mathrm{~d} \bar{\rho}_{5}}\right]_{+}$

25. $\left[\frac{d e_{a p .2}}{d \bar{\rho}_{6}}\right]_{+}$

26. $\left[\frac{d e_{\text {ap }, 1}}{d T_{5}}\right]_{+}$

27. $\left[\frac{\mathrm{de}_{\mathrm{vap} .2}}{\mathrm{dT}_{5}}\right]$

28. $\left[\frac{\mathrm{dT}}{\mathrm{Sat}, 1} \frac{\mathrm{d} \bar{\rho}_{5}}{\mathrm{~d}}\right]$

29. $\left[\frac{\mathrm{dT}_{\mathrm{Sa}, 2}}{\mathrm{~d} \bar{\rho}_{6}}\right]_{+}$

30. $\left[\frac{\mathrm{dT}_{\mathrm{Sat}, 1}}{\mathrm{dT}_{5}}\right]_{+}$

31. $\left[\frac{\mathrm{dT}_{\mathrm{Sat}, 2_{2}}}{\mathrm{dT}_{5}}\right]_{+}$

(For the SESAME option "on," replace the above M subscripts by the corresponding M' subscripts.)

\section{Arguments:}

NC - input: total number of cells in the vectors.

$\mathrm{RB}(\mathrm{NC}, 7) \quad$ - input: component macrodensities $\bar{\rho}_{\mathrm{m}+}$.

$\mathrm{T}(\mathrm{NC}, 5) \quad$ - input: component temperatures $T_{r+}$.

(If SESAME option "on," next two arguments:)

$\operatorname{ALP}(N C, 5) \quad$ - output: component volume fractions $\alpha_{r+}$. 
RHO(NC,7) - input: initial guess for component densities $\rho_{m+}$; output: component densities $\rho_{\mathrm{m}+}$.

$\operatorname{EOSV}(\mathrm{NC}, 15)$ - output: various quantities; see above.

DER(NC,31) - output: derivatives of various quantities; see above.

(If SESAME option "on," next three arguments:)

ER(NC,114) - auxiliary array.

LER(NC,9) - auxiliary array.

IER(NC,4) - auxiliary array.

Remarks: The dimension of all arrays is (MIJBP2,*).

Called Routines: If the SESAME option is "on": EOSPEQ, VSTOP, VSELOT, and some auxiliary routines. 
Purpose: Given the vectors of the component macrodensities $\bar{\rho}_{\mathrm{m}+^{\prime}} \mathrm{m}=1, \ldots, 7$, and of the component temperature $T_{r+}, r=1, \ldots, 5$, EOSS1 obtains the vectors of the component volume fractions $\alpha_{r+}, r=1, \ldots, 5$, of the specific internal energies $e_{r+}, r=1, \ldots, 4$, and of the following quantities (if the SESAME option is "off": from input constants or straightforwardly calculated; if the SESAME option is "on": under the assumption of mechanical equilibrium):

component-specific heat capacities:
1. $\left[\frac{\mathrm{de}_{3}}{\mathrm{dT}_{3}}\right]_{+}$
2. $\left[\frac{\mathrm{de}_{4}}{\mathrm{dT}_{4}}\right]_{+}$
3. $\left[\frac{\mathrm{de}_{\mathrm{G}}}{\mathrm{dT}_{5}}\right]_{+}$

\section{Arguments:}

NC - input: total number of cells in the vectors.*

$\mathrm{RB}(\mathrm{NC}, 7) \quad$ - input: component macrodensities $\bar{\rho}_{\mathrm{m}+}$.

$\mathrm{T}(\mathrm{NC}, 5) \quad$ - input: component temperatures $\mathrm{T}_{\mathrm{r}+{ }^{*}}{ }^{*}$

SIE(NC,5) - output: component specific-internal energies $e_{r+}$.

$\mathrm{CV}(\mathrm{NC}, 3) \quad$ - output: component specific heat capacities, see above.

ALP(NC,5) - oliciut: component volume fractions $\alpha_{r+}$.

(If SESAME option "on," next four arguments:)

RHO(NC,7) - input: initial guess for component densities $\rho_{m+i}$ output: component densities $\rho_{\mathrm{m}+}$.

ER(NC,114) - auxiliary array.

LER(NC,8) - auxiliary array.

$\operatorname{IER}(\mathrm{NC}, 4)$ - auxiliary array.

Remarks: The dimension of all arrays is (MIJBP2,*).

Called Routines: If the SESAME option is "on": EOSPEQ and some auxiliary routines.

\footnotetext{
"Instead of NC, IB, JB and IJB are used for SESAME "off."

** Instead of T, TEM is used for SESAME "off."
} 
Purpose: Given the vectors of the component macrodensities $\bar{\rho}_{\mathrm{m}+^{\prime}} \mathrm{m}=1, \ldots, 7$, and of the component temperature $T_{r+}, r=1, \ldots, 5$, EOSPD obtains the vectors of the component volume fractions $\alpha_{r+}, r=1, \ldots, 5$, of the component specific-internal energies $e_{r+}$, $e=1, \ldots, 4, G$, and of the following quantities (if the SESAME option is "off": from input constants or straightforwardly calculated; if the SESAME option is "on": under the assumption of mechanical ec dilibrium):

pressure derivatives with regard to the velocity-component macrodensities:
1. $\left[\frac{d p}{d \bar{\rho}_{L I}}\right]_{+}$
2. $\left[\frac{\mathrm{dp}}{\mathrm{d} \overline{\mathrm{p}}_{\mathrm{L} 2}}\right]_{+}$
3. $\left[\frac{\mathrm{dp}}{\mathrm{d} \bar{\rho}_{\mathrm{G}}}\right]_{+}$
SESAME off
I. $\left[\frac{d p}{d \bar{\rho}_{L 1}}\right]_{+}$
2. $\left.\left[\frac{\mathrm{dp}}{\mathrm{d} \bar{\rho}_{\mathrm{G}}}\right]_{+}\right\}$
SESAME on
4. $\left[\frac{\mathrm{dp}}{\mathrm{dT}_{5}}\right]_{+}$

Vapor mixture specific-internal energy derivatives with regard to the velocitycomponent macrodensities:
1. $\left[\frac{\mathrm{de}_{\mathrm{G}}}{\mathrm{d}_{\mathrm{L} 1}}\right]_{+}$
2. $\left[\frac{\mathrm{de}_{\mathrm{G}}}{\mathrm{d} \overline{\mathrm{P}}_{\mathrm{L} 2}}\right]_{+}$
3. $\left[\frac{d_{\mathrm{G}_{\mathrm{G}}}}{\mathrm{d} \bar{\rho}_{\mathrm{G}}}\right]_{+}^{+}$
SESAME off
$\left.\begin{array}{l}\text { 1. }\left[\frac{d e_{G}}{d \bar{\rho}_{\mathrm{L} 1}}\right]_{+} \\ \text {2. }\left[\frac{d e_{G}}{d \bar{\rho}_{\mathrm{G}}}\right]_{+}\end{array}\right\}$
SESAME on
4. $\left[\frac{\mathrm{de}_{\mathrm{G}}}{\mathrm{dT}_{5}}\right]_{+}$ 
SESAME "on" only:

Derivatives of various quantities, such as pressure $\mathrm{p}_{+}$, particle, liquid components and vapor mixture specific-internal energies $e_{r+}, r=2,3,4, G$, and vapor volume fraction $\alpha_{G+}$ :

1. $\left[\frac{\mathrm{dp}}{\mathrm{d} \bar{\rho}_{2}}\right]_{+}\left[\frac{\mathrm{de}}{\mathrm{d} \overline{\mathrm{\rho}}_{2}}\right]_{+}\left[\frac{\mathrm{de}}{\overline{d \bar{\rho}}}\right]_{+}\left[\frac{\mathrm{de}}{\mathrm{d} \overline{\bar{\rho}_{2}}}\right]_{+}\left[\frac{\mathrm{de}}{\mathrm{d} \bar{\rho}_{2}}\right]_{+}\left[\frac{\mathrm{d} \alpha_{\mathrm{G}}}{\mathrm{d} \bar{\rho}_{2}}\right]_{+}$

2. $\left[\frac{\mathrm{dp}}{\mathrm{d} \bar{\rho}_{3}}\right]_{+}\left[\frac{\mathrm{de}}{\mathrm{d} \bar{\rho}_{3}}\right]_{+}\left[\frac{\mathrm{de}}{\mathrm{d} \bar{\rho}_{3}}\right]_{+}\left[\frac{\mathrm{de}_{3}}{\mathrm{~d} \bar{\rho}_{3}}\right]_{+}\left[\frac{\mathrm{de}}{\mathrm{d} \bar{\rho}_{3}}\right]_{+}\left[\frac{\mathrm{d} \alpha_{G}}{\mathrm{~d} \bar{\rho}_{3}}\right]_{+}$

3. $\left[\frac{\mathrm{dp}}{\mathrm{d} \bar{\rho}_{4}}\right]_{+}\left[\frac{\mathrm{de} \mathrm{G}_{\bar{G}}}{\mathrm{~d} \bar{\rho}_{4}}\right]_{+}\left[\frac{\mathrm{de}}{\mathrm{d} \bar{\rho}_{4}}\right]_{+}\left[\frac{\mathrm{de}}{\mathrm{d} \bar{\rho}_{4}}\right]_{+}\left[\frac{\mathrm{de}}{\mathrm{d} \bar{\rho}_{4}}\right]_{+}\left[\frac{\mathrm{d} \alpha_{G}}{\mathrm{~d} \bar{\rho}_{4}}\right]_{+}$

4. $\left[\frac{\mathrm{dp}}{\mathrm{d} \bar{\rho}_{5}}\right]_{+}\left[\frac{\mathrm{de}_{\mathrm{G}_{5}}}{\mathrm{~d} \bar{\rho}_{5}}\right]_{+}\left[\frac{\mathrm{de}_{2}}{\mathrm{~d} \bar{\rho}_{5}}\right]_{+}\left[\frac{\mathrm{de}_{3}}{\mathrm{~d} \bar{\rho}_{5}}\right]_{+}\left[\frac{\mathrm{de}_{4}}{\mathrm{~d} \bar{\rho}_{5}}\right]_{+}\left[\frac{\mathrm{d} \alpha_{G}}{\mathrm{~d} \bar{\rho}_{5}}\right]_{+}$

5. $\left[\frac{\mathrm{dp}}{\mathrm{d} \bar{\rho}_{6}}\right]_{+}\left[\frac{\mathrm{de}}{\mathrm{d} \bar{\rho}_{6}}\right]_{+}\left[\frac{\mathrm{de}}{\mathrm{d} \bar{\rho}_{6}}\right]_{+}\left[\frac{\mathrm{de}}{\mathrm{d} \bar{\rho}_{6}}\right]_{+}\left[\frac{\mathrm{de}}{\mathrm{d} \bar{\rho}_{6}}\right]_{+}\left[\frac{\mathrm{d} \alpha_{G}}{\mathrm{~d} \bar{\rho}_{6}}\right]_{+}$

6. $\left[\frac{\mathrm{dp}}{\mathrm{d} \bar{\rho}_{7}}\right]_{+}\left[\frac{\mathrm{de}}{\mathrm{d} \bar{\rho}_{7}}\right]_{+}\left[\frac{\mathrm{de}}{\mathrm{d} \bar{\rho}_{7}}\right]_{+}\left[\frac{\mathrm{de}}{\mathrm{d} \bar{\rho}_{7}}\right]_{+}\left[\frac{\mathrm{de}}{\mathrm{d} \bar{\rho}_{7}}\right]_{+}\left[\frac{\mathrm{d} \alpha_{G}}{\mathrm{~d} \bar{\rho}_{7}}\right]_{+}$

7. $\left[\frac{\mathrm{dp}}{\mathrm{dT}_{2}}\right]_{+}\left[\frac{\mathrm{de}_{\mathrm{G}}}{\mathrm{dT}_{2}}\right]_{+}\left[\frac{\mathrm{de}_{2}}{\mathrm{dT}_{2}}\right]_{+}\left[\frac{\mathrm{de}_{3}}{\mathrm{dT}_{2}}\right]_{+}\left[\frac{\mathrm{de}_{4}}{\mathrm{dT}_{2}}\right]_{+}\left[\frac{\mathrm{d}_{\mathrm{G}}}{\mathrm{dT}_{2}}\right]_{+}$

8. $\left[\frac{\mathrm{dp}}{\mathrm{dT}_{3}}\right]_{+}\left[\frac{\mathrm{de}_{\mathrm{G}}}{\mathrm{dT}_{3}}\right]_{+}\left[\frac{\mathrm{de}_{2}}{\mathrm{dT}_{3}}\right]_{+}\left[\frac{\mathrm{de}_{3}}{\mathrm{dT}_{3}}\right]_{+}\left[\frac{\mathrm{de}_{4}}{\mathrm{dT}_{3}}\right]_{+}\left[\frac{\mathrm{d}_{\mathrm{G}}}{\mathrm{dT}_{3}}\right]_{+}$

9. $\left[\frac{\mathrm{dp}}{\mathrm{dT}_{4}}\right]_{+}\left[\frac{\mathrm{d}_{\cdot} \cdot \mathrm{j}}{\mathrm{dT}_{4}}\right]_{+}\left[\frac{\mathrm{de}_{2}}{\mathrm{dT}_{4}}\right]_{+}\left[\frac{\mathrm{de}_{3}}{\mathrm{dT}_{4}}\right]_{+}\left[\frac{\mathrm{de}_{4}}{\mathrm{dT}_{4}}\right]_{+}\left[\frac{\mathrm{d} \alpha_{\mathrm{G}}}{\mathrm{dT}_{4}}\right]_{+}$

10. $\left[\frac{\mathrm{dp}}{\mathrm{dT}_{5}}\right]_{+}\left[\frac{\mathrm{de}_{\mathrm{G}}}{\mathrm{dT}_{5}}\right]_{+}\left[\frac{\mathrm{de}_{2}}{\mathrm{dT}_{5}}\right]_{+}\left[\frac{\mathrm{de}_{3}}{\mathrm{dT}_{5}}\right]_{+}\left[\frac{\mathrm{de}_{4}}{\mathrm{dT}_{5}}\right]_{+}\left[\frac{\mathrm{d}_{\mathrm{G}}}{\mathrm{dT}_{5}}\right]_{+}$

\section{Arguments:}

NC

$\operatorname{ALP}(N C, 5) \quad$ - output: component volume fractions $\alpha_{\mathrm{r}+\cdot}$

$\mathrm{RB}(\mathrm{NC}, 7) \quad$ - input: component macrodensities $\bar{\rho}_{\mathrm{m}+}$.

RHO(NC,7) - input: initial guess for component densities $\rho_{m}+$. output: component densities $\rho_{\mathbf{m}+}$.

$\mathrm{T}(\mathrm{NC}, 5) \quad$ - input: component temperatures $T_{r+}$.

$\mathrm{P}(\mathrm{NC}) \quad$ - output pressure $\mathrm{p}_{+}$.

"Instead of NC, IB and JB are used for SESAME "off." 
(If SESAME option "off," next argument:)

SIEG(NC) - output: vapor mixture specific-internal energy $\mathrm{e}_{\mathrm{G}+}$.

(If SESAME option "on," next argument:)

SIE(NC,5) - output: vapor component and vapor mixture specific-internal energies $e_{r+}, r=1, \ldots, 4, G$.

PTEST(NC) - input: used if SESAME option "off" only, to force a singlephase treatment (if $<0$ ).

$\mathrm{DP}(\mathrm{NC}, 4) \quad$ - output: pressure derivatives; see above.

$\mathrm{DEG}(\mathrm{NC}, 4) \quad$ - output: vapor mixture specific-interrial energy derivatives; see above.

(If SESAME option "off," next argument:)
$\mathrm{ER}(\mathrm{NC}, 12)$
- auxiliary array.

(If SESAME option "on," next four arguments:)

DYDX(NC,6,10) - output: derivatives of various quantities; see above.

ER(NC,114) -auxiliary array.

$\operatorname{IER}(\mathrm{NC}, 4)$ - auxiliary array.

LER(NC,9) - auxiliary array.

Remarks: The dimension of the arrays is (MIJBP2,*) or $\left(\mathrm{MIJBP} 2,6,{ }^{*}\right)$, respectively.

Called Routines: If the SESAME option is "on": EOSPEQ, VSELOT, and some auxiliary routines. 
Purpose: SESINI fetches the 301 and 401 tables of the SESNM materials (SESNM $\equiv M N M A T$ ), identifiers that are specified in the MATER array, from the file specified by SESNAM and LSU, converts them into SI units, and stores them in the arrays provided for the tables.

Also, SESINI obtains from the saturation data tables of the materials the critical temperature $T_{C r t, M^{\prime}}$ and the critical pressures $\mathrm{PCrt}_{\mathrm{r}} \mathrm{M}^{\prime}, \mathrm{M}^{\prime}=3,2$.

Arguments:

FN

LUN

IFNT

NMAT

MIDS

IPRNT

WPRNT
LPRNT

- input: identical with SESNAM common variable.

- input: identical with LSU common variable.

- input: identical with SESTYP common variable.

- input: identical with SESNM common variable.

- input: identical with MATER common array.

- input: identical with SESPR common variable.

- input: identical with OUT common variable.

- input: identical with PRT common variable.

IERS(SESNM) - output: error code.

Remarks: For the definition of the above common variables and arrays see the remark in the introduction to this appendix.

Called Routines: FEXIST, CHKLUN, ASSIGN, RDFIL. 
Purpose: SPNINI derives the spinodal data of the SESNM material from the Vander-Waals-type data tables and uses them to override the default data in the spinodal data tables.

\section{Arguments:}

MER - input: dimension of ER array.

ER(MER,31) - auxiliary array.

Remarks: The spinodal data tables are generated for NSPNDL(M) temperatures equidistantly spaced between the second-lowest temperature value in the Van-derWaals-type table and the critical temperature. To generate the spinodal data, the Van-der-Waals-type isotherms for the above temperatures are sampled for their maxima and minima using a grid of NISOT density values between zero and the critical density.

Called Routines: SESEOS. 
Purpose: SESPRT prints the following tables as stored in the code arrays in the initialization phase of the code: tine Van-der-Waals-type or solid EOS data tables, the saturation or sublimation tables, the spinodal tables, the solid EOS tables, and the sublimation tables. The tables are printed in this order for each of the materials for which they are defined; the order of the materials is that of the substances "Solid 2, Solid 1, Liquid/Vapor 1, Liquid/Vapor 2, and Noncondensible Gas."

Arguments: None

Called Routines: TXTFLM. 
Purpose: Obtains from the Van-der-Waals-type data or solid EOS data tables of a given material $\mathbf{M}^{\prime}(\mathrm{m})$ the vectors of the pressure $\mathrm{PVan}, \mathrm{M}^{\prime}+$ or $\mathrm{PSId}, \mathrm{M}^{\prime}+$, respectively, and of its derivatives and/or of the specific internal energy eVan, $\mathrm{M}^{\prime}+$ or $\mathrm{eSId}_{2} \mathrm{M}^{\prime}+$, respectively, and of its derivatives (in SI units) as a function of the vectors of the densities $\rho_{+}$and of the temperature $T_{+}$(note that the derivatives are returned with regard to the logarithm of the independent variables):

1. PVan, $M^{\prime}+\left\{\rho_{+}, T_{+}\right\}$or PSld, $M^{\prime}+\left\{\rho_{+}, T_{+}\right\}$, respectively.

2. $\partial_{\ln \rho \mathrm{PVan}, \mathrm{M}^{\prime}+}\left(\rho_{+}, \mathrm{T}_{+}\right)$or $\partial_{\ln \rho \mathrm{PSId}, \mathrm{M}^{\prime}+}\left(\rho_{+}, \mathrm{T}_{+}\right\}$, respectively.

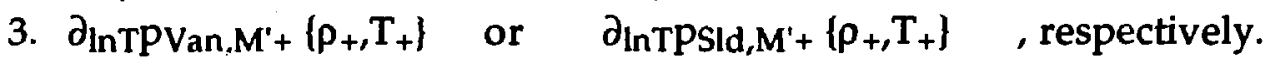

1. eVan, $M^{\prime}+\left\{\rho_{+}, T_{+}\right\}$or esld, $M^{\prime}+\left\{\rho_{+}, T_{+}\right\} \quad$, respectively.

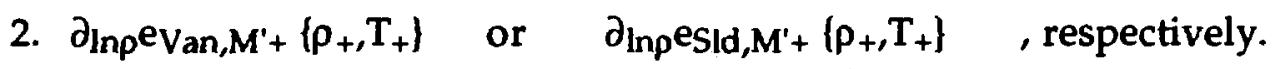

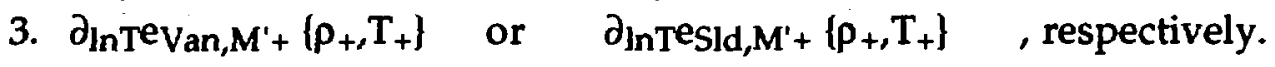

\section{Arguments:}

IREG

- input: component number $m$; for $m=2$ the solid EOS table is accessed; for $m=3, \ldots, 7$ the Van-der-Waals-type table is accessed.

XVALS(NC) - input: vector of densities $\rho_{+}$.

YVALS(NC) - input: vector of temperatures $T_{+}$.

NC - input: total number of cells in the vector.

IOPT - input: if IOPT =

1 or 3: calculate the pressure and its derivatives.

2 or 3: calculate the specific internal energy its derivatives.

$\operatorname{EOSP}(\mathrm{NC}, 3)$ - output: vectors of the pressure and its derivatives.

$\operatorname{EOSE}(\mathrm{NC}, 3)$ - output: vectors of the specific internal energy and of its derivatives.

$\mathrm{E}(\mathrm{NC}, 20) \quad$ - auxiliary array.

$\mathrm{IE}(\mathrm{NC}, 2) \quad$ - auxiliary array.

Remarks: Note that in SESEOS and in the called routines, the two-dimensional arrays are used as one-dimensional arrays, i.e., their elements are accessed consecutively. 
Called Routines: SRCHDF, BILIN, LINEAR, SRCH2D, BIQUAD, QUAD, SRCH3D, BIRATF, RATF. 
Purpose: Obtains from the saturation table of a given material $\mathrm{M}^{\prime}(\mathrm{m})$ the vectors of the following quantities (in SI units) as a function of the vector of the pressure $p_{+}$:
1. $\mathrm{T}_{\mathrm{Sat}, \mathrm{M}^{\prime}+}\left(\mathrm{p}_{+}\right\}$
2. $\partial_{\mathrm{p}} \mathrm{T}_{\mathrm{Sat}, \mathrm{M}^{\prime}+}\left\{\mathrm{p}_{+}\right\}$
3. $\mathrm{VVap}_{,} \mathrm{M}^{\prime}+\left\{\mathrm{p}_{+}\right\}$
4. $\partial \mathrm{TeVap}, \mathrm{M}^{\prime}+\left\{\rho_{+}\right\}$
5. eCon, $M^{\prime}+\left\{\rho_{+}\right\}$
6. $\partial_{\mathrm{T}} \mathrm{Con}, \mathrm{M}^{\prime}+\left\{\rho_{+}\right\}$
7. $\rho$ Vap, $M^{\prime}+\left\{\rho_{+}\right\}$
8. $\partial_{\mathrm{T} \rho \mathrm{Vap}_{,} \mathrm{M}^{\prime}+}\left\{\rho_{+}\right\}$
9. $\rho$ Con, $M^{\prime}+\left\{\rho_{+}\right\}$
10. $\partial \mathrm{T} \rho \operatorname{Con}_{,} \mathrm{M}^{\prime}+\left[\rho_{+}\right]$

\section{Arguments:}

NREG

PIN(NX)

$\operatorname{SATV}(N X, 10)$

NX

ICTRL

$\mathrm{E}(\mathrm{NX}, 16)$

$\mathrm{IE}(\mathrm{NX}, 2)$
- input: component number $\mathrm{m}$.

- input: vector of pressure $\rho_{+}$.

- output: vectors of the resulting quantities - see above [array dimension SATV (MIJBP2,*)].

- input: total number of cells in the vectors.

- input: selects output quantity numbers;

if 1: calculate quantities 1,2;

if 2: calculate quantities $1, \ldots, 6$;

if 3: calculate quantities $1, \ldots, 10$.

- auxiliary array [array dimension E(MIJBP2,*i)].

- auxiliary array [array dimension IE(MIJBP2,*)].

Remarks: The saturation temperature resulting from the interpolation of quantity 1 is used in the interpolation of the other quantities.

The temperature-pressure relation is interpolated as $1 / \mathrm{T}_{\mathrm{Sat}_{,} \mathrm{M}^{+}+}$over $\ln \mathrm{p}_{+}$. The temperature-saturated-vapor relation is interpolated as $\ln \rho$ Vap, $M^{\prime}+$ over $1 / T_{+}$. All other quantities are interpolated linearly as they stand. 
If the arguments p+ exceed the table boundaries, the values of the last entries, i.e., the critical values, are returned for the underivated quantities, and the derivatives are set to zero.

Called Routines: LAGRAN, URANUS. 
Purpose: Obtains from the saturation table of a given material $M^{\prime}(m)$ the vectors of the following quantities (in SI units) as a function of the vector of the temperature $\mathrm{T}_{+}$:

1. pSat, $^{\prime}+\left\{\mathrm{T}_{+}\right\}$

2. PVap, $M^{\prime}+\left\{T_{+}\right\}$

3. $\rho$ Con, $\mathbf{M}^{\prime}+\left\{\mathrm{T}_{+}\right\}$

\section{Arguments:}

NREG

$\operatorname{TEM}(\mathrm{NX})$

$\operatorname{SATV}(N X, 3)$

NX

ICTRL

$\mathrm{E}(\mathrm{NX}, 12)$

IE(NX,2)
- input: component number $\mathrm{m}$.

- input: vector of temperature $T_{+}$.

- output: vectors of the resulting quantities, see above (array dimension SATV(MIJBP2,*)).

- input: total number of cells in the vectors.

- input: selects output quantity numbers;

if 1 : calculate quantity 1 ;

if 2: calculate quantities $1, \ldots, 3$.

- auxiliary array (array dimension E(MIJBP $\left.2,{ }^{*}\right)$ ).

- auxiliary array (array dimension IE(MIJBP2,*)).

Remarks: The temperature-saturated-vapor relation is interpolated as $\ln \rho$ Vap, $\mathbf{M}^{+}+$ over $1 / T_{+}$. The temperature-saturated-vapor relation is interpolated as $\ln \rho V_{a p, M^{+}}$ over $1 / T_{+}$. All other quantities are interpolated linearly as they stand.

If the arguments $T_{+}$exceed the table boundaries, the values of the last entries, i.e., the critical values, are returned for the underivated quantities, and the derivatives are set to zero.

Called Routines: LAGRAN, URANUS. 
Purpose: Obtains from the saturation or sublimation table of a given material $M(m)$ the vectors of the following quantities (in SI units) as a function of the vector of the temperature $T_{+}$:

1. e Con, $\mathbf{M}^{+}\left\{\mathrm{T}_{+}\right\}$or 1. esus, $\mathrm{M}^{+}\left\{\mathrm{T}_{+}\right\}$, respectively.

2. $\partial_{\mathrm{T}} \mathrm{C}_{\mathrm{Con}, \mathrm{M}^{\prime}+}\left\{\mathrm{T}_{+}\right\}$or 2. $\partial_{\mathrm{T}} \mathrm{Sus}_{\mathrm{S}} \mathrm{M}^{\prime}+\left\{\mathrm{T}_{+}\right\}$, respectively.

3. PCon, $\mathrm{M}^{\prime}+\left\{\mathrm{T}_{+}\right\}$or 3. PSus, $\mathrm{M}^{\prime}+\left\{\mathrm{T}_{+}\right\}$, respectively.

\section{Arguments:}

NREG - input: component number $\mathrm{m}$.

TEM(NX) - input: vector of temperature $\mathrm{T}+$.

$\operatorname{SATV}(N X, 3) \quad$ - output: vectors of the resulting quantities, see above [array dimension SATV(MIJBP2,*)].

NX - input: total number of cells in the vectors.

ICTRL - input: selects output quantity numbers;

if 1 : calculate quantities 1,2 ;

if 2: calculate quantities $1, \ldots 3$.

$\mathrm{E}(\mathrm{NX}, 10) \quad$ - auxiliary array [array dimension $\mathrm{E}\left(\mathrm{MIJBP} 2{ }^{*}\right)$ ].

IE(NX,2) - auxiliary array [array dimension IE(MIJBP2,*)].

Remarks: If the arguments $T_{+}$exceed the table boundaries, the values of the last entries, i.e., the critical values, are returned for the underivated quantities, and the derivatives are set to zero.

Ca11ed Routines: LAGRAN. 
Purpose: Obtains from the saturation or sublimation table of a given material $\mathrm{M}^{\prime}(\mathrm{m})$ the vectors of the following quantities (in SI units) as a function of the vector of the (condensated liquid) specific internal energy $e_{+}$:
1. $\mathrm{T}_{\mathrm{Sat}, \mathrm{M}^{\prime}+}\left\{\mathrm{e}_{+}\right\}$or
1. $\mathrm{T}_{\mathrm{Sub}, \mathrm{M}^{\prime}+}\left\{\mathrm{e}_{+}\right\}$, respectively
2. deT $\mathrm{TSt}_{\mathrm{S}, \mathrm{M}^{\prime}+}\left\{\mathrm{e}_{+}\right\}$or
2. $\partial_{\mathrm{e}} \mathrm{T}_{\mathrm{Sub}, \mathrm{M}^{\prime}+}\left\{\mathrm{e}_{+}\right\}$, respectively
3. $\rho_{C o n,} M^{\prime}+\left\{e_{+}\right\}$or
3. PSus, $M^{\prime}+\left\{e_{+}\right\}$
respectively

\section{Arguments:}

NREG - input: component number $\mathrm{m}$.

$\operatorname{ENER}(N X) \quad$ - input: vector of specific internal energy $e_{+}$.

$\operatorname{SATV}(\mathrm{NX}, 3)$ - output: vectors of the resulting quantities, see above [array dimension SATV(MIJBP2,*)].

NX - input: total number of cells in the vectors.

$\mathrm{E}(\mathrm{NX}, 10) \quad$ - auxiliary array [array dimension $\mathrm{E}\left(\mathrm{MIJBP} 2{ }^{*}\right)$ ].

$\operatorname{IE}(\mathrm{NX}, 2) \quad$ - auxiliary array [array dimension IE(MIJBP2,*)].

Remarks: The saturation or sublimation temperature resulting from the interpolation of quantity 1 is used in the interpolation of the other quantities.

All quantities are interpolated linearly as they stand.

If the arguments $e_{+}$exceed the table boundaries, the values of the last entries, i.e., the critical values, are returned for the underivated quantities, and the derivatives are set to zero.

Called Routines: LAGRAN. 
Purpose: Obtains from the spinodal table of a given material $\mathbf{M}^{\prime}(\mathrm{m})$ the vectors of the following quantities (in SI units) as a function of the vector of the temperature $T_{+}$:
1. $\mathrm{pSpg}, \mathrm{M}^{\prime}+\left\{\mathrm{T}_{+}\right\}$

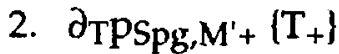
3. $\rho \mathrm{Spg}, \mathrm{M}^{\prime}+\left\{\mathrm{T}_{+}\right\}$
4. $\mathrm{PSpl}, \mathrm{M}^{+}+\left\{\mathrm{T}_{+}\right\}$
5. $\partial \mathrm{TPSpl}, \mathrm{M}^{\prime}+\left\{\mathrm{T}_{+}\right\}$
6. $\rho \mathrm{Spl}, \mathrm{M}^{\prime}+\left\{\mathrm{T}_{+}\right\}$

\section{Arguments:}

NREG

- input: component number m.

PORT(NX) - input: vector of temperature $\mathrm{T}+$.

SPNDV(NX,6) - output: vectors of the resulting quantities, see above [array dimension SPNDV(MNX,*)].

NX

- input: total number of cells in the vectors.

ICTRL

- input: not used

MNX

- input: dimension of array SPNDV, E, IE.

$\mathrm{E}(\mathrm{NX}, 13)$

- auxiliary array [array dimension $\mathrm{E}(\mathrm{MNX}, *)]$.

$\mathrm{IE}(\mathrm{NX}, 2)$

- auxiliary array [array dimension IE(MNX,*)].

Remarks: The quantities 1 and 2 are interpolated as $\ln \mathrm{PSpg}, \mathrm{M}_{+}$over $1 / \mathrm{T}_{+}$. Similarly, the quantity 3 is interpolated as $\ln \rho_{5 p g, M+}$ over $1 / T+$. The quantities 4,5 , and 6 are interpolated linearly as they stand.

If the arguments $T_{+}$exceed the table boundaries, the values of the last entries, i.e., the critical values, are returned for the underivated quantities, and the derivatives are set to zero.

Called Routines: LAGRAN, URANUS. 
Purpose: Linearly interpolates in a given table to obtain a vector $y_{+}\left(x_{+}\right)$of function values and a vector $\partial_{x+} y_{+}$of its derivatives.

\section{Arguments:}

$X(N P) \quad$ - input: table of $x$-values.

$Y(N P) \quad$ - input: table of $y$-values.

XVAL(NC) - input: vector of arguments $x_{+}$.

YARG(NC,2) - output: vector of results $y_{+}$and $\partial_{x+} y_{+}$ [dimension YARG(MIJBP2, $\left.{ }^{*}\right)$ ].

$\mathrm{E}(\mathrm{NC}, 5)$ - auxiliary array [dimension $\left.\mathrm{E}\left(\mathrm{MIJBP} 2,{ }^{*}\right)\right]$.

$\operatorname{IE}(\mathrm{NC}, 2)$ - auxiliary array [dimension IE(MIJBP2,*)];

$\mathrm{IE}(\mathrm{NC}, 1)$ and IE $(\mathrm{NC}, 2)$ contain the vectors of the relevant lower and upper table positions, respectively, either as output (if ICTRL $=0$ ) or as input (if ICTRL $=1$ ).

NP -input: total number of table entries.

NC - input: total number of cells in the vectors.

IDEG - not used.

ICTRL - input: if 0 : find positions in the table and store in $\operatorname{IE}(\mathrm{NC}, 1)$ and $\mathrm{IE}(\mathrm{NC}, 2)$;

if 1: take positions in the table from $\operatorname{IE}(\mathrm{NC}, 1)$ and $\operatorname{IE}(\mathrm{NC}, 2)$.

Called Routines: None. 
Purpose: Nonlinearly interpolates in a given table to obtain a vector $y_{+}\left(x_{+}\right)$of function values and a vector $\partial_{x+} y_{+}$of its derivatives. If IXVAL $=2$, ln y is linearly interpolated over $1 / x$, if $\mathrm{IXVAL}=1$, and $1 / y$ is linearly interpolated over $\ln x$.

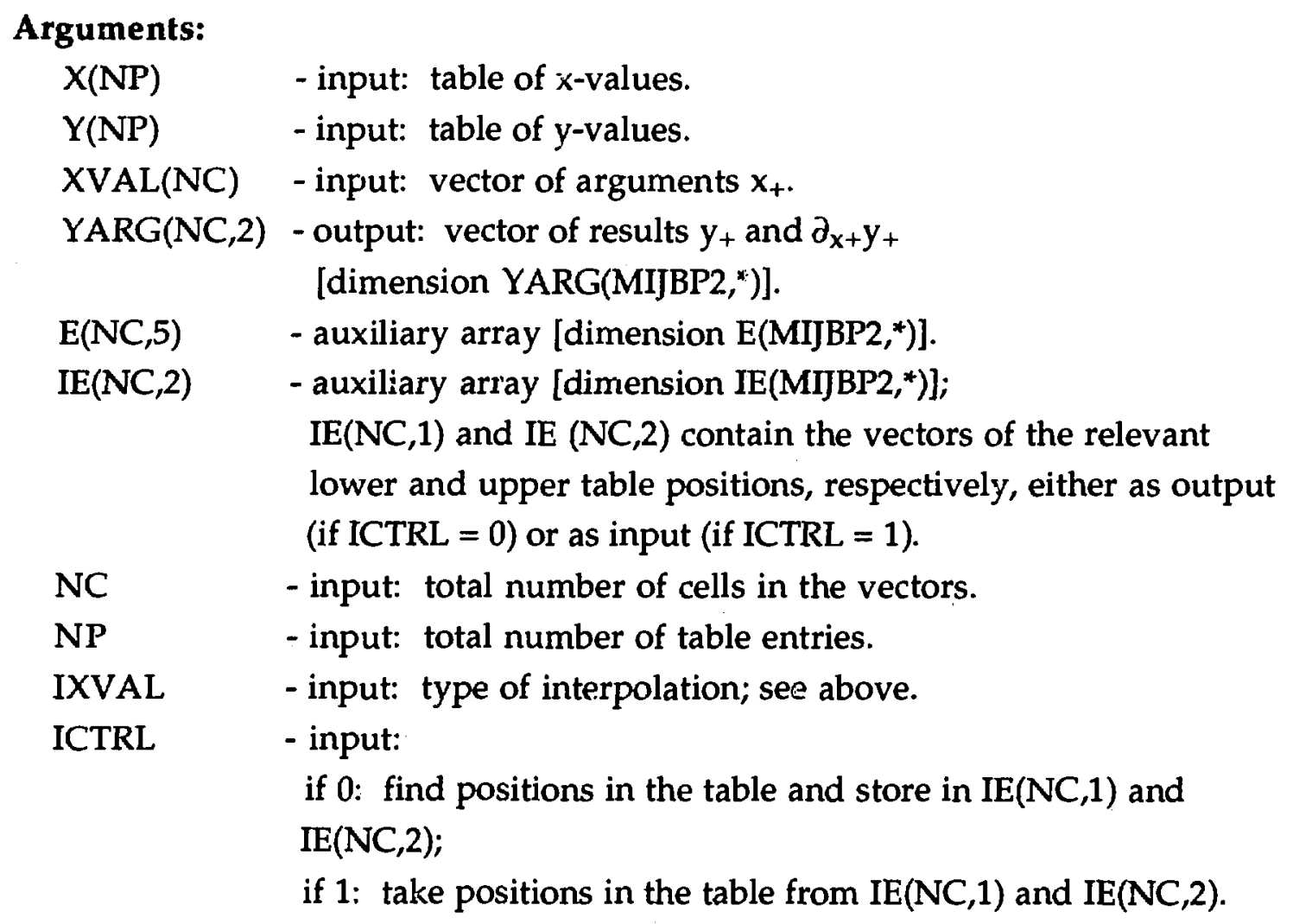

Called Routines: None. 
Purpose: For given vectors of the component temperatures $T_{r+}$, macrodensities $\bar{\rho}_{\mathrm{m}+}$ and vapor component-specific heat capacities $c_{v, M}+$ THEPHY obtain the vectors of the component thermal conductivities $k_{r+}, r=1, \ldots, 5$, dynamic viscosities $\mu_{\mathrm{r}+}, \mathrm{r}=3,4,5$, and vapor mixture-specific heat capacity at constant pressure $c_{\mathrm{p}+}$.

\section{Arguments:}

NMC - input: total number of cells in the vectors.

$\mathrm{T}(\mathrm{NMC}, 5) \quad$ - input: vector of component temperature $T_{\mathrm{r}+}$.

$R B(N M C, 7)$ - input: vector of component macrodensities $\bar{\rho}_{\mathrm{m}+}$.

RHO - not used.

$\mathrm{P} \quad-$ not used.

$\mathrm{CV}(\mathrm{NMC}, 3)$ - input: vector of vapor component specific heat capacities $\mathrm{C}_{\mathrm{V}}, \mathrm{M}^{+}$.

$\mathrm{KP}(\mathrm{NMC}, 5)$ - output: vector of component thermal conductivities $\mathrm{k}_{\mathrm{r}+\text { - }}$.

VIS(NMC,3) - output: vector of component dynamic viscosities $\mu_{r+}$.

$\mathrm{CP}(\mathrm{NMC}) \quad$ - output: vectrr of vapor mixture specific heat capacity at constant pressure $c_{p+}$.

Remarks: The dimension of all arrays is (MIJBP2,*).

Called Routines: None. 


\section{APPENDIX C \\ ROUTINE FLOWCHARTS}

This appendix is not contained within the volume but forms a separate document, which is available upon request. 


\section{APPENDIX D \\ VARIABLE NAMES AND SYMBOLS}

Table D.I lists the common variables and arrays and the parameters related to the EOS and to the thermophysical properties.

In column 1 of the table, the preprocessor option is indicated under which the variable, array, or parameter is included in the code (if in parenthesis, the variable or array always is included in the code but used under the indicated option only).

In column 2, the name of the variable, array, or parameter is listed, along with the array dimension, where applicable.

In column 3, the symbol of the corresponding quantity used in this volume of the AFDM documentation is given, along with the occurring values of the subscripts, where applicable. (For the SESAME option "on," the M subscripts must be replaced by the corresponding $M$ ' subscripts, where applicable.) Numerical values are given for parameters.

In column 4, the COMMON name (upper-case letters) of the variable or array or the Comdeck name (lower-case letters) of the PARAMETER statement, respectively, is listed. In one instance, the equivalenced common array name is listed instead.

In column 5, a character indicates if the variable or array is assigned explicit or default input (I: input required under the option specified in column 1; A: input required for SESAME off only). Input from the tables is not indicated.

Table D.II lists the symbols of the quantities appearing in this volume.

In column 1 of the table, the symbols are listed in alphabetical order, Latin symbols preceding the Greek symbols. (For the SESAME option "on," the M subscripts must be replaced by the corresponding $\mathrm{M}^{\prime}$ subscripts, where applicable.)

In column 2, the variable or array names carrying the symbolic material or component subscripts rather than their dimension are given.

In column 3, a character indicates the type of the quantity:

- I for input constants needed under all options;

- A for input constants needed under the SESAME option"off" only;

- Q for input constants needed under the KQQM1 option "on" only;

- K for input constants needed under the KQQM2 option "on" only;

- D for constants derived from input constants; 
TABLE D.I

COMMON VARIABLES AND ARRAYS

\begin{tabular}{|c|c|c|c|c|c|}
\hline \multirow[t]{22}{*}{ Option } & Name & \multicolumn{2}{|l|}{ Symbol } & Common & Input \\
\hline & MNMAT & \multicolumn{2}{|l|}{3} & Dimen & \\
\hline & MNDEN & \multicolumn{2}{|l|}{7} & Dimen & \\
\hline & MNENER & \multicolumn{2}{|l|}{5} & Dimen & \\
\hline & MNVEL & \multicolumn{2}{|l|}{3} & Dimen & \\
\hline & ALPHAO & \multicolumn{2}{|l|}{$\alpha_{0}$} & EOSPAR & I \\
\hline & EPSP & \multicolumn{2}{|l|}{$\varepsilon_{\mathfrak{p}}$} & INPUT & I \\
\hline & EPSTG & \multicolumn{2}{|l|}{$\varepsilon_{e}$} & EOSCOM & I \\
\hline & TCRIT(MNMAT) & $\mathrm{T}_{\mathrm{Crt}, \mathrm{M}}$ & $M=1,2,3$ & EOSPARa & A \\
\hline & RTCRIT(MNMAT) & $1 / \mathrm{T}_{\mathrm{Crt}, \mathrm{M}}$ & $M=1,2$ & EOSPAR & \\
\hline & PCRIT(MNMAT) & PCrt, M & $M=1,2$ & EOSPAR & \\
\hline & HFM(MNMAT) & $h_{f, M}$ & $\mathrm{M}=1,2$ & EOSPAR $^{b}$ & A \\
\hline & RHFM(MNMAT) & $1 / h_{f, M}$ & $M=1,2$ & EOSPAR $\mathbf{b}$ & \\
\hline & TSOLUS(MNMAT) & $\mathrm{T}_{\text {Sol,M }}$ & $M=1,2$ & EOSPAR & I \\
\hline & TLIQUS(MNMAT) & $\mathrm{T}_{\text {Liq, }, \mathrm{M}}$ & $M=1,2$ & EOSPAR & I \\
\hline & ESOLM(MNMAT) & eSol,M & $M=1,2$ & EOSPARb & \\
\hline & ELIQM(MNMAT) & $e_{\text {Liq, }} M$ & $M=1,2$ & EOSPAR $\mathbf{b}$ & \\
\hline & EZERO(MNENER) & $e_{r}^{0}$ & $r=1,2,3,4$ & EOSPAR & \\
\hline & TZERO(MNENER) & $T_{r}^{0}$ & $r=1,2,3,4$ & EOSPAR & \\
\hline & TL175 & $0.75 \times \mathrm{T}_{\text {Liq }} \mathrm{a}$ & & EOSPAR & \\
\hline & TL275 & $0.75 \times \mathrm{T}_{\mathrm{Liq},}{ }^{b}$ & & EOSPAR & \\
\hline & TSUPM(MNMAT) & $\Delta T_{\text {Sup }, M}$ & $M=1,2$ & EOSPAR & I \\
\hline (NOPHASE off:) & TDHOM & $\Delta \mathrm{T}_{h}$ & & MTFC & \\
\hline \multirow[t]{3}{*}{ (SESAME off:) } & HROR(MNDEN) & $\rho_{\mathrm{m}}$ & $\mathrm{m}=1, \ldots, 7$ & EOSCOM & \\
\hline & & \multicolumn{3}{|c|}{$=\rho S, 2, \rho S, 1, \rho L, 1, \rho L, 2, \ldots$} & I \\
\hline & RUGM(MNMAT) & $\mathbf{R}_{\mathbf{M}}$ & $M=1,2,3$ & EOSPARc & $\mathbf{I}$ \\
\hline (SESAME off:) & ECRIT(MNMAT) & $e_{C r t, M}$ & $M=1,2,3$ & EOSCOM & I \\
\hline (SESAME off:) & CSM(MNMAT) & $\mathrm{c}_{\mathrm{vS}, \mathrm{M}}$ & $M=1,2$ & EOSCOM & I \\
\hline
\end{tabular}

aThese quantities are not used for $M=3$ if SESAME "on." bThese quantities are not used for $M=2$ if SESAME "on." CRUGM is also required for SESAME "on," along with KQQM1 on. 
TABLE D.I (CONT.)

\begin{tabular}{|c|c|c|c|c|c|}
\hline Option & Name & Symbol & & Common & Input \\
\hline (SESAME off:) & CLM(MNMAT) & $c_{v L, M}$ & $M=1,2$ & EOSCOM & I \\
\hline (SESAME off:) & CGM(MNMAT) & $c_{v G, M}$ & $M=1,2,3$ & EOSCOM & I \\
\hline (SESAME off:) & HLGM(MNMAT) & $\mathrm{h}_{\mathrm{lg}, \mathrm{M}}$ & $M=1,2$ & EOSCOM & \\
\hline (SESAME off:) & CVLM(MNMAT) & $\mathrm{CS}_{1}, \mathrm{C}_{\mathrm{L}, 1}, \mathrm{C}_{\mathrm{L}, 2}$ & & EOSCOM & I \\
\hline (SESAME off:) & RCVLSQ(MNMAT) & $1 / \mathrm{c}_{\mathrm{S}, 1}^{2}, 1 / \mathrm{c}_{\mathrm{L}, 1}^{2}, 1 / \mathrm{c}_{\mathrm{T}}$ & $\begin{array}{ll}2 \\
L, 2\end{array}$ & EOSCOM & \\
\hline (SESAME off:) & TSTAR(MNMAT) & $\mathrm{T}_{\mathrm{M}}^{*}$ & $M=1,2$ & EOSPAR & I \\
\hline (SESAME off:) & RTSTAR(MNMAT) & $1 / T_{M}^{*}$ & $M=1,2$ & EOSPAR & \\
\hline (SESAME off:) & PSTAR(MNMAT) & $P_{M}^{*}$ & $M=1,2$ & EOSCOM & $\mathbf{I}$ \\
\hline (SESAME off:) & ESUPM(MNMAT-1) & & & EOSPAR & \\
\hline SESAME off: & MREGS & 5 & & Xeosdim & \\
\hline SESAME off: & SESNM & $\overline{\mathbf{M}^{\prime}}$ & & XEOSPRM & \\
\hline SESAME on: & MAXR3 & 150 & & Xeosdim & \\
\hline SESAME on: & MAXT3 & 150 & & Xeosdim & \\
\hline SESAME on: & MAXT4 & 100 & & Xeosdim & \\
\hline SESAME on: & NSPIN & 16 & & Xeosdim & \\
\hline SESAME on: & NRTAB(MNDEN) & $M^{\prime}(m)$ & $\mathrm{m}=1, \ldots, 7$ & XEOSPRM & I \\
\hline SESAME on: & MATER(MREGS) & & & XEOSPRM & I \\
\hline SESAME on: & NR3(MREGS) & & & XEOSPRM & \\
\hline ESAME on: & NT3(MREGS) & & & XEOSPRM & \\
\hline ESAME on: & NT4(MREGS) & & & XEOSPRM & \\
\hline KQQM2 on:) & RCGAS & $\mathbf{R}^{\mathbf{0}}$ & & EOSPAR & $\mathbf{I}$ \\
\hline NOPHASE off:) & WMOLE(MNMAT) & $\mathbf{M}_{\mathbf{M}}$ & $M=1,2,3$ & EOSPAR & I \\
\hline NOPHASE off:) & RWMOLE(MNMAT) & $1 / \mathrm{M}_{\mathrm{M}}$ & $M=1,2,3$ & EOSPAR & \\
\hline KQQM2 on:) & NATOM(MNMAT) & $\mathrm{n}_{\mathrm{A}, \mathrm{M}}$ & $M=1,2,3$ & EOSPAR & I \\
\hline KQQM2 on:) & SIGLJ(MNMAT) & $\sigma_{M}$ & $\mathrm{M}=1,2,3$ & EOSPAR & $\mathbf{I}$ \\
\hline KQQM2 on:) & EPSOK(MNMAT) & $\varepsilon / k_{M}$ & $M=1,2,3$ & EOSPAR & $\mathbf{I}$ \\
\hline KQQM2 on:) & REPSOK(MNMAT) & $k / \varepsilon_{M}$ & $\mathrm{M}=1,2,3$ & EOSPAR & I \\
\hline KQQM2 on: & SLNL1 & $\sigma_{\mathrm{LN}, 1}$ & & TPPROP & $\mathbf{I}$ \\
\hline GQM2 on: & SLN2 & $\sigma_{\mathrm{LN}, 2}$ & & TPPROP & I \\
\hline KQQM2 on: & SLFLL1 & $\sigma_{\mathrm{LFL}, 1}$ & & TPPROP & I \\
\hline KQQM2 on: & SLFLL2 & $\sigma_{\mathrm{LFL}, 2}$ & & TPPROP & I \\
\hline
\end{tabular}


TABLE D.I (CONT.)

\begin{tabular}{|c|c|c|c|c|c|}
\hline Option & Name & Symbol & & Common & Input \\
\hline KQQM2 on: & SLFEL1 & $\sigma_{\mathrm{LFE}, 1}$ & & TPPROP & I \\
\hline KQQM2 on: & SLFEL2 & $\sigma_{\mathrm{LFE}, 2}$ & & TPPROP & $\mathbf{I}$ \\
\hline KQQM2 on: & SLEXL1 & $\sigma_{\mathrm{LEX}, 1}$ & & TPPROP & I \\
\hline KQQM2 on: & SLEXL2 & $\sigma_{\mathrm{LEX}, 2}$ & & TPPROP & I \\
\hline KQQM2 on: & TSLCL1 & $\mathrm{T}_{G L C, 1}$ & & TPPROP & I \\
\hline KQQM2 on: & TSLCL2 & $\mathrm{T}_{\mathrm{GLC}, 2}$ & & TPPROP & I \\
\hline KQQM2 on: & SLLIN & $\sigma_{\text {LLIN }}$ & & TPPROP & I \\
\hline KQQM2 on: & SLLIF & $\sigma_{\text {LLIF }}$ & & TPPROP & I \\
\hline KQQM2 on: & TSLLIR & TSLLIR $_{\text {S }}$ & & TPPROP & I \\
\hline KQQM2 on: & CKAG(3) & $\mathrm{C}_{\mathrm{KAG}, \mathrm{M}}$ & $M=1,2,3$ & TPPROP & I \\
\hline KQQM2 on: & CKBG(3) & $\mathrm{C}_{\mathrm{KBG}, \mathrm{M}}$ & $M=1,2,3$ & TPPROP & I \\
\hline KQQM2 on: & CKCG(3) & $\mathrm{C}_{\mathrm{KCG}, \mathrm{M}}$ & $M=1,2,3$ & TPPROP & I \\
\hline KQQM2 on: & CMUA(2) & $\mathrm{C}_{\mathrm{MUA}, \mathrm{M}}$ & $M=1,2$ & TPPROP & I \\
\hline KQQM2 on: & CMUB(2) & $\mathrm{C}_{\mathrm{MUB}, \mathrm{M}}$ & $M=1,2$ & TPPROP & I \\
\hline KQQM2 on: & CKA(4) & $\mathrm{C}_{\mathrm{KAm}}$ & $\mathrm{m}=1,2,3,4$ & TPPROP & I \\
\hline KQQM2 on: & $\mathrm{CKB}(4)$ & $\mathrm{C}_{\mathrm{KBm}}$ & $\mathrm{m}=1,2,3,4$ & TPPROP & I \\
\hline KQQM1 on: & $\mathrm{KC}(4)$ & $\mathbf{k}_{\mathbf{r}}$ & $r=2,3,4,5$ & TPPROP & \\
\hline KQQM1 on: & KPS & $\mathrm{k}_{2}$ & & $\equiv \mathrm{KC}(1)$ & I \\
\hline KQQM1 on: & KPL(1) & $k_{3}$ & & $\equiv \mathrm{KC}(2)$ & I \\
\hline KQQM1 on: & KPL(2) & $k_{4}$ & & $\equiv \mathrm{KC}(3)$ & I \\
\hline KQQM1 on: & KPG & $\mathrm{k}_{5}$ & & $\equiv \mathrm{KC}(4)$ & I \\
\hline KQQM1 on: & XMU(MNMAT) & $\mu_{\mathbf{r}}$ & $r=3,4,5$ & MXFC & $\mathbf{I}$ \\
\hline
\end{tabular}

- T for constants derived from the tables;

- $\mathrm{S}$ for variable quantities taken from the tables;

Two alternatives separated by a slash correspond to the SESAME option "off/ on," respectively.

In column 4, the physical units of the quantities are stated, a hyphen indicating dimensionless quantities. 


\section{TABLE D.II \\ SYMBOLS OF THE QUANTITIES}

Symbol

Name

Type

Unit

Material and vapor mixture-specific heat capacities at constant volume:

\begin{tabular}{|c|c|c|c|}
\hline$c_{v S, M}$ & $\operatorname{CSM}(\mathrm{M})$ & A & $\mathrm{J} \mathrm{kg}^{-1} \mathrm{~K}^{-1}$ \\
\hline$c_{\mathrm{vL}, \mathrm{M}}$ & CLM(M) & A & $\mathrm{J} \mathrm{kg}^{-1} \mathrm{~K}^{-1}$ \\
\hline$c_{V G, M}$ & $\mathrm{CGM}(\mathrm{M})$ & A & $\mathrm{J} \mathrm{kg}^{-1} \mathrm{~K}$ \\
\hline$c_{v}$ & & & $\mathrm{~J} \mathrm{~kg}^{-1} \mathrm{~K}^{-1}$ \\
\hline
\end{tabular}

Material and vapor mixture-specific heat capacities at constant pressure:

$\begin{array}{ll}c_{\mathrm{pL}, \mathrm{M}} & \mathrm{J} \mathrm{kg}^{-1} \mathrm{~K}^{-1} \\ \mathrm{c}_{\mathrm{pG}, \mathrm{M}} & \mathrm{J} \mathrm{kg}^{-1} \mathrm{~K}^{-1} \\ c_{\mathrm{p}} & \mathrm{J} \mathrm{kg}^{-1} \mathrm{~K}^{-1}\end{array}$

Solid and liquid material sonic velocities:

$\begin{array}{lllll}\mathrm{CS}_{1} M & \mathrm{CVLM}(\mathrm{M}) & \mathrm{M}=1 & \mathrm{~A} & \mathrm{~m} \mathrm{~s}^{-1} \\ \mathrm{CL}_{2} \mathrm{M} & \mathrm{CVLM}(\mathrm{M}+1) & \mathrm{M}=1,2 & \mathrm{~A} & \mathrm{~m} \mathrm{~s}^{-1}\end{array}$

Parameters in thermal conductivity relations:

$\begin{array}{llll}C_{K A G, M} & \text { CKAG(M) } & K & W m^{-1} K^{-1} \\ C_{K B G, M} & \text { CKBG(M) } & K & W m^{-1} K^{-2} \\ C_{K C G, M} & \text { CKCG(M) } & K & W m^{-1} K^{-3} \\ C_{K A m} & C K A(m) & K & W m^{-1} K^{-1} \\ C_{K B m} & C K B(m) & K & W m^{-1} K^{-2}\end{array}$

Parameters in dynamic viscosity relations:

$\begin{array}{llll}\text { C MUA,M }_{\text {M }} & \text { CMUA(M) } & \mathrm{K} & \mathrm{kg} \mathrm{m}^{-1} \mathbf{~ s}^{-1} \\ \text { C }_{\text {MUB,M }} & \text { CMUB(M) } & \mathrm{K} & \mathrm{K}\end{array}$

Density-component- and energy-component-specific-internal energies:

\begin{tabular}{|c|c|c|}
\hline eM & & \\
\hline$e_{r}$ & & \\
\hline$e^{0}$ & EZERO(r) & $\mathrm{D} / \mathrm{T}$ \\
\hline
\end{tabular}




\section{TABLE D.II (CONT.)}
Symbol
Name
Type
Unit

Material solidus, liquidus, condensated liquid, saturated vapor, and critical specific internal energies:

\begin{tabular}{|c|c|c|c|}
\hline esol,M & ESOLM(M) & $\mathrm{D} / \mathrm{T}$ & $\mathrm{J} \mathrm{kg}^{-1}$ \\
\hline$e_{\text {Liq, }}$, & ELIQM(M) & $\mathrm{D} / \mathrm{T}$ & $\mathrm{J} \mathrm{kg}^{-1}$ \\
\hline eCon, $M$ & & $/ S$ & $\mathrm{~J} \mathrm{~kg}^{-1}$ \\
\hline eVap,M & & $/ \mathrm{S}$ & $\mathrm{J} \mathrm{kg}^{-1}$ \\
\hline$e_{C r t, M}$ & ECRIT(M) & $\mathrm{A} / \mathrm{T}$ & $\mathrm{J} \mathrm{kg}^{-1}$ \\
\hline
\end{tabular}

Material-specific internal heats of vaporization:
$e_{\text {lg, } M}$
/S
$\mathrm{J} \mathrm{kg}^{-1}$

Material Van-der-Waals-type and solid EOS-specific internal energies:
eVan, $M^{\prime}$
$S$
$\mathrm{J} \mathrm{kg}^{-1}$
esld, $M^{\prime}$
$\mathrm{S}$
$\mathrm{J} \mathrm{kg}^{-1}$

Material sublimation specific-internal energies (solid side):

$$
\begin{array}{lll}
\text { esus, } M^{\prime} & S & J_{k g}^{-1}
\end{array}
$$

Material-specific heats of fusion and vaporization enthalpies:
$h_{f, M}$
HFM(M)
A/T
$\mathrm{J} \mathrm{kg}^{-1}$
$h_{\lg , M}$
HLGM(M)
$\mathrm{D} / \mathrm{S}$
$\mathrm{J} \mathrm{kg}^{-1}$

Mesh column number and mesh cell number:

i

ij

Density-component-specific enthalpies:

$\mathrm{i}_{\mathrm{m}}$

$\mathrm{J} \mathrm{kg}^{-1}$

Material condensated liquid and saturated vapor-specific enthalpies:

$\begin{array}{lcc}\text { iCon,M } & / S & \mathrm{~J} \mathrm{~kg}^{-1} \\ \text { iVap,M } & / S & \mathrm{~J} \mathrm{~kg}^{-1}\end{array}$




\section{TABLE D.II (CONT.)}

Symbol

Name

Type

Unit

Mesh row number:

j

Energy-component thermal conductivities:
$k_{r}$
$\mathrm{KC}(\mathrm{r}-1)$
Q
$W m^{-1} K^{-1}$

Density-component subscript, material subscript, and material index number:

$\mathrm{m}$
$\mathrm{M}$
$\mathrm{M}^{\prime}$

Material molecular weight:
$\mathrm{M}_{\mathrm{M}}$
WMOLE(M)
K
$\mathrm{kg} \mathrm{kmol}^{-1}$

Material number of gas atoms per molecule:
$\mathrm{n}_{\mathrm{A}, \mathrm{M}}$
$\operatorname{NATOM}(\mathrm{M})$
K

Total pressure, density-component and energy-component partial pressures:

$\mathrm{p}$

$\mathrm{N} \mathrm{m}^{-2}$

$P_{m}$

$\mathrm{N} \mathrm{m}{ }^{-2}$

$\mathrm{p}_{\mathrm{r}}$

$\mathrm{N} \mathrm{m}^{-2}$

Input cell pressure and noncondensible gas partial pressure:
$\mathrm{p}_{\text {cell }}^{\mathrm{i}}$
I
$\mathrm{N} \mathrm{m}^{-2}$
$p_{7}^{i}$
I
$\mathrm{N} \mathrm{m}^{-2}$

Material saturation, vapor, and liquid spinodal limit and critical pressure:

$\begin{array}{llll}\text { PSat, } M & & \text { /S } & \mathrm{N} \mathrm{m}^{-2} \\ \text { PSpg, } M^{\prime} & & \mathrm{S} & \mathrm{N} \mathrm{m}^{-2} \\ \text { PSpl,M' } & & \mathrm{S} & \mathrm{N} \mathrm{m}^{-2} \\ \text { PCrt,M } & \text { PCRIT(M) } & \mathrm{D} / \mathrm{T} & \mathrm{N} \mathrm{m}^{-2}\end{array}$




\section{TABLE D.II (CONT.)}

Symboi Name Type Unit

Material pressure coefficients in saturation relations:
$\mathrm{P}_{\mathrm{M}}^{*}$
PSTAR(M)
A
$\mathrm{N} \mathrm{m}^{-2}$

Material Van-der-Waals-type and solid EOS pressures:

$\begin{array}{lll}\text { PVan, } M^{\prime} & S & \mathrm{~N} \mathrm{~m}^{-2} \\ \text { PSId, } M^{\prime} & \mathrm{S} & \mathrm{N} \mathrm{m}^{-2}\end{array}$

Material sublimation pressure:

PSub,M'

$\mathrm{S} \quad \mathrm{N} \mathrm{m}^{-2}$

Velocity-component subscript:

q

Energy-component:

$\mathbf{r}$

Universal gas constant:
$\mathrm{R}^{\mathbf{0}}$
RCGAS
$\mathrm{K}$
$\mathrm{J} \mathrm{kmol}^{-1} \mathrm{~K}^{-1}$

Material gas constants:
$\mathbf{R}_{\mathbf{M}}$
RUGM(M)
$\mathbf{A}^{1}$
$\mathrm{J} \mathrm{kg}^{-1} \mathrm{~K}^{-1}$

Energy-component temperature and input temperatures:

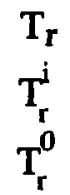

$T_{r}$
$T_{r}^{j}$
$T_{r}^{0}$

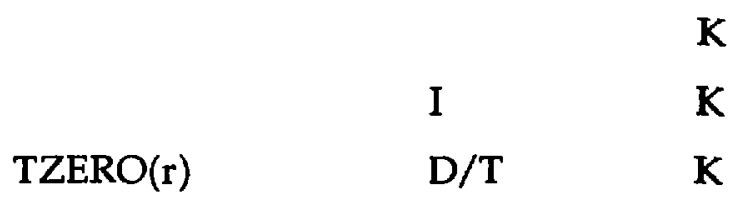

K

K

K

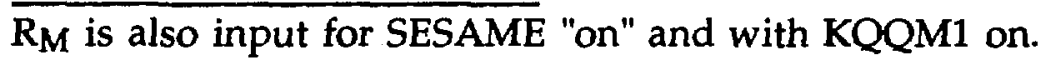




\section{TABLE D.II (CONT.)}

Symbol Name Type Unit

Material solidus, liquidus, saturation, critical, and sublimation temperatures and superheat:

\begin{tabular}{|c|c|c|}
\hline $\mathrm{T}_{\text {Sol,M }}$ & TSOLUS(M) & I \\
\hline $\mathbf{T}_{\text {Liq, }, M}$ & TLIQUS(M) & I \\
\hline $\mathrm{T}_{\text {Sat, } M}$ & & /S \\
\hline $\mathrm{T}_{\mathrm{Crt}, \mathrm{M}}$ & TCRIT(M) & $\mathrm{A} / \mathrm{T}$ \\
\hline $\mathrm{T}_{\text {Sub,M' }}$ & & $S$ \\
\hline$\Delta \mathrm{T}_{\text {Sup }, \mathrm{M}}$ & TSUPM(M) & 1 \\
\hline
\end{tabular}

Derived quantities:
$0.75 \times \mathrm{T}_{\text {Liq1 }}$
TL175
D K
$0.75 \times \mathrm{T}_{\text {Liq2 }}$
TL275
D
K
$\Delta \mathrm{T}_{h}$
TDHOM
$\mathrm{D} / \mathrm{T}$
K

Material temperature coefficients in saturation relation:
$\mathrm{T}_{\mathrm{M}}^{*}$
TSTAR(M)
A
K

Temperature parameters in surface tension relations:

$\begin{array}{llll}\text {T GLC,M }_{\text {TSLCL1, TSLCL2 }} & \text { K } & \text { K } \\ \text { TSLLIR }_{\text {SSLLIR }} & \text { TS } & \text { K }\end{array}$

Energy-component volume fractions and input volume fractions:<smiles>[Te][Te]</smiles>

Minimum vapor and effective gas volume fractions:

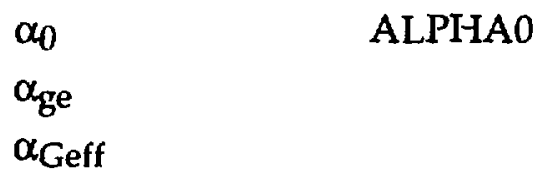 K $\mathrm{K}$ K $\mathrm{K}$ $\mathrm{K}$ $\mathrm{K}$ 


\section{TABLE D.II (CONT.)}
Symbol
Name
Type
Unit

Limits for the macrodensities of "missing components" and of "non-present" components:
$\varepsilon_{x}$
XSMALL
$\mathrm{kg} \mathrm{m}^{-3}$
$\varepsilon$
SMALL
$\mathrm{kg} \mathrm{m}^{-3}$

Convergence criteria for mechanical equilibrium and temperature inversion iterations:

$\begin{array}{llll}\varepsilon_{\mathrm{p}} & \text { EPSP } & \mathrm{I} & \mathrm{N} \mathrm{m}^{-2} \\ \varepsilon_{e} & \text { EPSTG } & \mathrm{I} & \mathrm{J} \mathrm{kg}^{-1}\end{array}$

Material Lennard-Jones parameters:
$\varepsilon / \mathrm{k}_{\mathrm{M}}$
EPSOK(M)
$\mathrm{K}$
$\mathrm{K}$

Energy-component dynamic viscosities:
$\mu_{\mathrm{r}}$
$\mathrm{XMU}(\mathrm{r}-2)$
Q
$\mathrm{kg} \mathrm{m}^{-1} \mathrm{~s}^{-1}$

Density-component densities and input densities:

$\begin{array}{llll}\rho_{m} & & & \mathrm{~kg} \mathrm{~m}^{-3} \\ \rho_{S, M} & \text { HROR }[\mathrm{m}(\mathrm{M})] & \mathrm{A} / \mathrm{S} & \mathrm{kg} \mathrm{m}^{-3} \\ \rho_{L, M} & \text { HROR }[\mathrm{m}(\mathrm{M})] & \mathrm{A} / \mathrm{S} & \mathrm{kg} \mathrm{m}^{-3}\end{array}$

Material condensated liquid, saturated vapor, liquid, and vapor spinodal limit and critical densities:

$\begin{array}{lll}\text { PCon,M } & \text { /S } & \mathrm{kg} \mathrm{m}^{-3} \\ \text { PVap,M } & / S & \mathrm{~kg} \mathrm{~m}^{-3} \\ \text { PSpl, }^{\prime} & \mathrm{S} & \mathrm{kg} \mathrm{m}^{-3} \\ \text { PSpg, }^{\prime} & \mathrm{S} & \mathrm{kg} \mathrm{m}^{-3} \\ \text { PCrt,M }^{\prime} & \mathrm{D} / \mathrm{T} & \mathrm{kg} \mathrm{m}^{-3}\end{array}$

Material sublimation densities (solid side):

$$
\text { PSus, } M^{\prime}
$$

$S$

$\mathrm{kg} \mathrm{m}^{-3}$ 
TABLE D.II (CONT.)

Symbol

Name

Type

Unit

Density-component macrodensities:

$$
\bar{\rho}_{\mathrm{m}}
$$

$\mathrm{kg} \mathrm{m}^{-3}$

Velocity-component macrodensities

$$
\bar{\rho}_{\mathrm{q}}
$$

$\mathrm{kg} \mathrm{m}^{-3}$

Material molecular diameters:

$\sigma_{\mathrm{M}} \quad \operatorname{SIGLJ}(\mathrm{M})$

K

$\AA$

Surface tension parameters:

$\sigma_{L N, M}$
$\sigma_{L F L, M}$
$\sigma_{L F E, M}$
$\sigma_{L E X, M}$
$\sigma_{L L I N}$
$\sigma_{L L I F}$

SLNL1, SLNL2

K

$\mathrm{kg} \mathrm{s}^{-2}$

SLFLL1, SLFLL2

$\mathrm{K}$

$\mathrm{kg} \mathrm{s}^{-2} \mathrm{~K}^{-1}$

SLFEL1, SLFEL2

$\mathrm{K}$

$\mathrm{kg} \mathrm{s}^{-2}$

SLEXL1, SLEXL2

$\mathrm{K}$

SLLIN

$\mathrm{K}$

$\mathrm{kg} \mathrm{s}^{-2}$

SLLIF

$\mathrm{K}$

$\mathrm{kg} \mathrm{s}^{-2} \mathrm{~K}^{-2}$ 


\section{APPENDIXE \\ SAEOS DATA}

For the SAEOS, the values for the material subscripts $M$ are rigidly assigned to the components (see Table A.I).

Table E.I shows the default input and input derived (the last three lines of the table) values (in SI units) of the AFDM code for the quantities entering the simplified analytic EOS (see Chap. II). $\mathrm{T}_{\mathrm{Crt}, \mathrm{M}}, \mathrm{e}_{\mathrm{Crt}, \mathrm{M}}$, and $\mathrm{PCrt}, \mathrm{M}$ for $\mathrm{M}=1,2$ do not enter the EOS directly but are used elsewhere in the code; the same is true for $\Delta \mathrm{T}_{\text {Sup, }} \mathrm{M}$. The quantities for $M=3$ for which no values are printed are not needed by the code, though default and explicit input is provided for.

TABLE E.I

SAEOS DEFAULT DATA

\begin{tabular}{|c|c|c|c|}
\hline $\mathbf{M}$ & 1 (fuel) & 2 (steel) & 3 (sodium vapor) \\
\hline $\mathbf{R}_{\mathbf{M}}$ & 25.55 & 127.92 & 361 \\
\hline$\rho S, M$ & 9100 & 7400 & \\
\hline$P_{L}, M$ & 8000 & 6000 & \\
\hline $\mathrm{T}_{\text {Sol,M }}$ & 3023 & 1670 & \\
\hline $\mathrm{T}_{\text {Lig, }} \mathrm{M}$ & 3063 & 1700 & \\
\hline $\mathrm{P}_{\mathrm{M}}^{*}$ & $2.07 \times 10^{12}$ & $1.338 \times 10^{11}$ & \\
\hline $\mathrm{T}_{M}^{*}$ & $6.1255 \times 10^{4}$ & $4.337 \times 10^{4}$ & \\
\hline $\mathrm{T}_{\mathrm{Crt}_{\mathrm{r}, \mathrm{M}}}$ & 10600 & 8000 & 2508 \\
\hline$e_{C r t, M}$ & $6.003 \times 10^{6}$ & $6.057 \times 10^{6}$ & $3.2604 \times 10^{6}$ \\
\hline $\mathrm{CS}, \mathrm{M}$ & 2500 & & \\
\hline $\mathfrak{C L}_{\mathrm{L}, \mathrm{M}}$ & 2000 & 2000 & \\
\hline $\mathcal{C}_{\mathrm{vS}, \mathrm{M}}$ & 638 & 639 & \\
\hline $\mathrm{C}_{\mathrm{vL}, \mathrm{M}}$ & 504 & 750 & \\
\hline $\mathrm{C}_{\mathrm{v}} \mathrm{C}, \mathrm{M}$ & 511 & 492 & 543.4 \\
\hline$h_{f, M}$ & $2.76 \times 10^{5}$ & $2.6 \times 10^{5}$ & \\
\hline$\Delta \mathrm{T}_{\text {Sup }, \mathrm{M}}$ & 10 & 2 & \\
\hline $\mathrm{PCrt}, \mathrm{M}$ & $6.4015 \times 10^{9}$ & $5.9161 \times 10^{8}$ & \\
\hline$e_{\text {Liq, }} \mathbf{M}$ & $2.2047 \times 10^{6}$ & $1.3271 \times 10^{6}$ & \\
\hline$h_{l g, M}$ & $1.5651 \times 10^{6}$ & $5.5479 \times 10^{6}$ & \\
\hline
\end{tabular}

The universal gas constant $R_{0}$ is defaulted to 8315 . 


\section{APPENDIX F \\ TEOS DATA}

In this chapter, numerical values of the SESAMESO library tables (see Sec. III.E.4) are displayed for the default materials fuel, steel, and sodium. All quantities are given in SI units. Contrary to the SAEOS, there is no fixed assignment between the material indices $\mathbf{M}^{\prime}$ and the components, but the default assignments are indicated (see Table A.I).

The liquid and vapor substance data originate from the SESAME EOS data library; modification thereof is indicated by footnotes. The solid substance data were supplied by KfK (see Sec. III.E.3).

This information is intended to illustrate the construction and interdependence of the data rather than to commit to their quality. It is recognized that the accuracy of the SESAME data is questionable. An update utilizing the best available data must be performed if the SESAME option of the AFDM couie is to be used for other than formal test runs.

\section{Fuel (Liquid and Vapor)}

Material identifier: 2451. Default material index: $\mathbf{M}^{\prime}=3$.

a. Van-der-Waals-type pressure $p_{V a n, k, l}\left\{\rho_{k}, T_{1}\right\}$ and specific internal energy eVan,, $\mathbf{l}\left[\rho_{k}, T_{l}\right]$ :

$\rho_{\mathbf{k}}: \mathrm{NR3}()=91$ entries, logarithmically spaced apart from the first one:

$$
\begin{aligned}
& \rho_{1}=0 ., \quad \rho_{2}=0.10968, \ldots, \quad \rho_{91}=10968 .{ }^{*} \\
& \rho_{k+1}=\rho_{k} \Delta \rho, \quad k=2, \ldots, 90, \quad \Delta \rho=1.1381
\end{aligned}
$$

$\mathrm{T}_{1}: \mathrm{NT} 3()=50$ entries, linearly spaced:

$$
\begin{aligned}
& \mathrm{T}_{1}=297.084, \quad \ldots, \quad \mathrm{T}_{50}=17407.3 \\
& \mathrm{~T}_{\mathrm{l}+1}=\mathrm{T}_{1}+\Delta \mathrm{T}, \quad \mathrm{l}=1, \ldots, 49, \quad \Delta \mathrm{T}=349.188
\end{aligned}
$$

b. Saturation properties PSat $k\left\{T_{k}\right\}$, $\rho \operatorname{Vap~} k\left\{T_{k}\right\}$, $\rho_{\text {con } k}\left\{T_{k}\right\}$, eVap $k\left\{T_{k}\right\}$, e Con $k\left\{T_{k}\right\}$ :

\footnotetext{
${ }^{*}$ The $\rho_{2}$ entry (see next two pages) should be added to the table for the SESAMESO library to avoid birational interpolation difficulties at the boundary.
} 
$T_{k}: N T 4()=38$ entries, linearly spaced apart from the last one:

$$
\begin{aligned}
& \mathrm{T}_{1}=297.084, \quad \ldots, \quad \mathrm{T}_{37}=12867.8, \quad \mathrm{~T}_{38}=\mathrm{T}_{\mathrm{Crt}}=13530.2 \\
& \mathrm{~T}_{\mathrm{k}+1}=\mathrm{T}_{\mathrm{k}}+\Delta \mathrm{T}, \quad \mathrm{k}=1, \ldots, 36, \quad \Delta \mathrm{T}=349.188
\end{aligned}
$$

c. Spinodal properties PSpg k $\left\{T_{k}\right\}, \operatorname{PSpl~k}\left\{T_{k}\right\}, \operatorname{PSpg~k}\{T k\}, \operatorname{PSpl~k~}\left\{T_{k}\right\}$ :

$T_{k}: \operatorname{NSPNDL}()=16$ entries, linearly spaced:

$$
\begin{aligned}
& \mathrm{T}_{1}=646.272, \quad \ldots, \quad \mathrm{T}_{16}=\mathrm{T}_{\mathrm{Crt}}=13530.2 \\
& \mathrm{~T}_{\mathrm{k}+1}=\mathrm{T}_{\mathrm{k}}+\Delta \mathrm{T}, \quad \mathrm{k}=1, \ldots, 15, \quad \Delta \mathrm{T}=858.929
\end{aligned}
$$

\section{1.b. Fuel (Solid)}

Material identifier: 12451. Default material index: $M^{\prime}=5$.

a. Solid EOS pressure PSId $k, 1\left\{\rho_{k}, T_{l}\right\}$ and specific internal energy esId $k, 1\left\{\rho_{k}, T_{l}\right\}$ :

$\rho_{\mathrm{k}}: \mathrm{NR3}()=18$ entries, irregularly spaced:

$$
\rho_{1}=9593.7 ., \quad \cdots, \quad \rho_{18}=10967 \text {. }
$$

$T_{1}:$ NT3( ) $=20$ entries, spaced in steps of 100 . or 200. .

$$
\mathrm{T}_{1}=300 ., \ldots, \mathrm{T}_{20}=3600 \text {. }
$$

b. Sublimation properties pSub $k\left\{T_{k}\right\}$, PSus k $\left\{T_{k}\right\}$, eSus $k\left\{T_{k}\right\}$ :

$T_{k}: N T 4()=21$ entries, spaced in steps of 100. or 200. apart from the first one:

$$
\mathrm{T}_{1}=0 ., \quad \mathrm{T}_{2}=300 ., \quad \ldots, \mathrm{T}_{21}=3600 .
$$

\section{2. a. Steel (Liquid and Vapor)}

Material identifier: 2450. Default material index: $\mathbf{M}^{\prime}=2$.

a. Van-der-Waals-type pressure $p_{V a n} k_{,}\left\{\rho_{k}, T_{1}\right\}$ and specific internal energy eVan $k, 1\left\{\rho_{k}, T_{1}\right\}$ :

$\rho_{k}=N R 3()=92$ entries, logarithmically spaced apart from the first two: 


$$
\begin{aligned}
& \rho_{1}=0 ., \quad \rho_{2}=7.87 \times 10^{-8}, \quad \rho_{3}=0.0787, \quad \ldots, \quad \rho_{92}=7870 . \\
& \rho_{k+1}=\rho_{k} \Delta \rho, \quad k=3, \ldots, 91, \quad \Delta \rho=1.1381
\end{aligned}
$$

$T_{1}: N T 3()=50$ entries, linearly spaced:

$$
\begin{aligned}
& T_{1}=297.084, \quad \ldots, \quad T_{50}=11604.8 \\
& T_{1+1}=T_{1}+\Delta T, \quad l=1, \ldots, 49, \quad \Delta T=230.770
\end{aligned}
$$

b. Saturation properties pSat k $\left\{T_{k}\right\}$, $\rho \operatorname{Vap~} k\left\{T_{k}\right\}, \rho \operatorname{Con} k\left\{T_{k}\right\}$, eVap k $\left\{T_{k}\right\}$, eCon k $\left\{T_{k}\right\}$ :

$T_{\mathbf{k}}: \mathrm{NT4}()=36$ entries, linearly spaced apart from the last one:

$$
\begin{aligned}
& \mathrm{T}_{1}=297.084, \quad \ldots, \quad \mathrm{T}_{35}=8143.29, \quad \mathrm{~T}_{36}=\mathrm{T}_{\mathrm{Crt}}=8251.48 \\
& \mathrm{~T}_{\mathrm{k}+1}=\mathrm{T}_{\mathrm{k}}+\Delta \mathrm{T}, \quad \mathrm{k}=1, \ldots, 34, \quad \Delta \mathrm{T}=230.770
\end{aligned}
$$

c. Spinodal properties pSpg k $\left\{T_{k}\right\}, \operatorname{pSpl~k}\left\{T_{k}\right\}, \rho$ Spg k $\left\{T_{k}\right\}, \rho S p l k\left\{T_{k}\right\}$ :

$T_{k}:$ NSPNDL( ) $=16$ entries, linearly spaced:

$$
\begin{aligned}
& T_{1}=527.855, \quad \ldots, \quad T_{16}=T_{C r t}=8251.48 \\
& T_{k+1}=T_{k}+\Delta T, \quad k=1, \ldots, 15, \quad \Delta T=514.908
\end{aligned}
$$

\section{2.b. Steel (Solid)}

Material identifier: 12450 . Default material index: $M^{\prime}=4$.

a. No solid EOS pressure and specific internal energy is specified (the steel liquid and vapor Van-der-Waals-type data are used instead to fill in this part of the table).

b. Sublimation properties pSub $k\left\{T_{k}\right\}$, PSus $k\left(T_{k}\right\}$, eSus $k\left(T_{k}\right)$ :

$T_{k}: N T 4()=21$ entries, spaced in steps of 50. or 100. apart from the first two:

$$
T_{1}=0 ., \quad T_{2}=297.08, \quad T_{3}=400 ., \quad \ldots, T_{21}=2000 \text {. }
$$

\footnotetext{
*The P2 $_{2}$ entry has been added to the table for the SESAMESO library to avoid birational intermolation difficulties at the boundary.
} 


\section{3.a. Sodium (Liquid and Vapor)}

Material identifier: 2448. Default material index: $M^{\prime}=1$.

a. Van-der-Waals-type pressure $P V_{a n}, 1,\left\{\rho_{k}, T_{l}\right\}$ and specific internal energy eVan $k_{,},\left\{\rho_{k}, T_{l}\right\}$ :

$$
\begin{aligned}
& \rho_{k}=\operatorname{NR} 3()=102 \text { entries, logarithmically spaced apart from the first two: } \\
& \rho_{1}=0 ., \quad \rho_{2}=1.011 \times 10^{-8}, \quad \rho_{3}=0.1011, \quad \ldots, \quad \rho_{102}=1314.3 . * \\
& \rho_{k+1}=\rho_{k} \Delta \rho, \quad k=3, \ldots, 101, \quad \Delta \rho=1.1004
\end{aligned}
$$

$T_{1}: N T 3()=25$ entries, first range linearly spaced, second range logarithmically spaced:

$$
\begin{aligned}
& \mathrm{T}_{1}=0, \quad \ldots, \quad \mathrm{T}_{17}=4061.85 \\
& \mathrm{~T}_{1+1}=\mathrm{T}_{\mathrm{l}}+\Delta \mathrm{T}, \quad \mathrm{l}=1, \ldots, 16, \quad \Delta \mathrm{T}=253.865 \\
& \mathrm{~T}_{17}=4061.85, \quad \ldots, \quad \mathrm{T}_{25}=10154.6 \\
& \mathrm{~T}_{\mathrm{l}+1}=\mathrm{T}_{1} \Delta \mathrm{T}, \quad \mathrm{l}=1, \ldots, 24, \quad \Delta \mathrm{T}=1.1213
\end{aligned}
$$

b. Saturation properties pSat $k\left\{T_{k}\right\}$, $\operatorname{Vap~} k_{k}\left\{T_{k}\right\}$, $\rho_{\text {Con } k}\left\{T_{k}\right\}$, eVap $k\left\{T_{k}\right\}$, eCon $k\left\{T_{k}\right\}$ (not used for default material assignment):

$T_{k}: N T 4()=11$ entries, linearly spaced:

$$
\begin{aligned}
& \mathrm{T}_{1}=0 ., \quad \ldots, \quad \mathrm{T}_{11}=\mathrm{T}_{\mathrm{Crt}}=2538.58 \\
& \mathrm{~T}_{\mathrm{k}+1}=\mathrm{T}_{\mathrm{k}}+\Delta \mathrm{T}, \quad \mathrm{k}=1, \ldots, 10, \quad \Delta \mathrm{T}=253.858
\end{aligned}
$$

c. Spinodal properties pSpg k $\left\{T_{k}\right\}, \operatorname{pSpl~k}\left\{T_{k}\right\}, \rho S p g k\left\{T_{k}\right\}, \rho S p l k\left\{T_{k}\right\}$ (not used for default material assignment):

$T_{k}:$ NSPNDL( ) $=16$ entries, linearly spaced:

$$
\begin{aligned}
& T_{l}=253.865, \quad \ldots, \quad T_{16}=T_{C r t}=2538.58 \\
& T_{k+1}=T_{k}+\Delta T, \quad k=1, \ldots, 15, \quad \Delta T=152.314
\end{aligned}
$$

No solid sodium material is provided in the library tables.

\footnotetext{
*The $\rho_{2}$ entry has been added to the table for the SESAMESO library to avoid birational interpolation difficulties at the boundary.
} 


\section{APPENDIX G}

\section{THERMOPHYSICAL PROPERTIES, DATA, AND PLOTS}

TABLE G.I

$\begin{array}{lcccccccc} & \begin{array}{c}\mathbf{T}_{\mathbf{C r t}} \\ (\mathrm{K})\end{array} & \begin{array}{c}\mathbf{T}_{\boldsymbol{\sigma L C}} \\ (\mathrm{K})\end{array} & \begin{array}{c}\sigma_{\mathrm{LN}} \\ \left(\mathrm{kg} / \mathrm{s}^{2}\right)\end{array} & \begin{array}{c}\sigma_{\text {LFL }} \\ \left(\mathrm{kg} / \mathrm{s}^{2}-\mathrm{K}\right)\end{array} & \begin{array}{c}\sigma_{\text {LFE }} \\ \left(\mathrm{kg} / \mathrm{s}^{2}\right)\end{array} & \sigma_{\text {LEX }} & & \\ \mathrm{UO}_{2} & 10600 & 2500 & 1.298 & 2.43 \times 10^{-4} & 1.35275 & 2.5 & \mathrm{~L} 1 & \mathrm{UO}_{2} \\ \mathrm{SS} & 8000 & 3000 & 1.846 & 4.00 \times 10^{-4} & 2.40862 & 2.8 & \text { L2 } & \end{array}$

\section{TABLE G.II}

$\begin{array}{ccccc} & \begin{array}{c}\text { TSLLIR } \\ (\mathrm{K})\end{array} & \begin{array}{c}\sigma_{\text {LLIN }} \\ \left(\mathrm{kg} / \mathrm{s}^{2}\right)\end{array} & \begin{array}{c}\sigma_{\text {LLIF }} \\ \left(\mathrm{kg} / \mathrm{s}^{2}-\mathrm{K}^{2}\right)\end{array} & \\ \mathrm{UO}_{2}-\mathrm{SS} & 3120 & 0.025 & 7.678 \times 10^{-7} & \text { L1 - L2 }\end{array}$

TABLE G.III

$\begin{array}{lccc} & \text { CMUA } & \text { C MUB } & \\ & (\mathrm{kg} / \mathrm{m}-\mathrm{s}) & (\mathrm{K}) & \\ \mathrm{UO}_{2} & 9.88 \times 10^{-4} & 4620 & \text { L1 } \\ \mathrm{SS} & 2.536 \times 10^{-4} & 5492 & \text { L2 }\end{array}$

\section{TABLE G.IV}

$\begin{array}{ccc}\mathrm{C}_{\mathrm{KA}} & \mathrm{C}_{\mathrm{KB}} & \\ (\mathrm{W} / \mathrm{m}-\mathrm{K}) & \left(\mathrm{W} / \mathrm{m}-\mathrm{K}^{2}\right) & \\ 20.0 & 0 & \mathrm{~S} \\ 0.95 & 6.0 \times 10^{-4} & \mathrm{P} \\ 5.6 & 0 & \mathrm{~L} 1 \\ 12.4 & 3.3 \times 10^{-3} & \text { L2 }\end{array}$




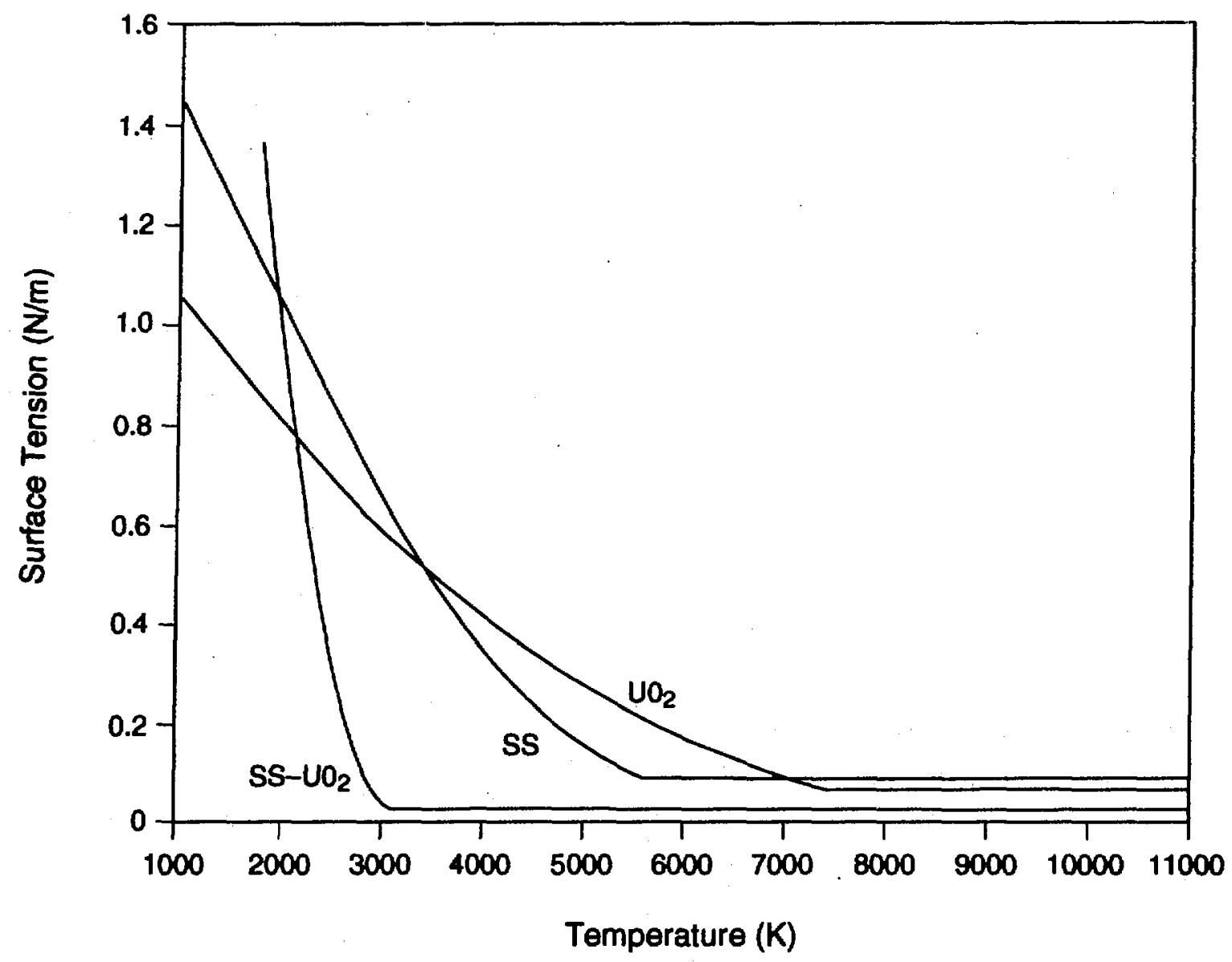

Fig. G.1.

Surface tension of stainless steel and $\mathrm{UO}_{2}$. 


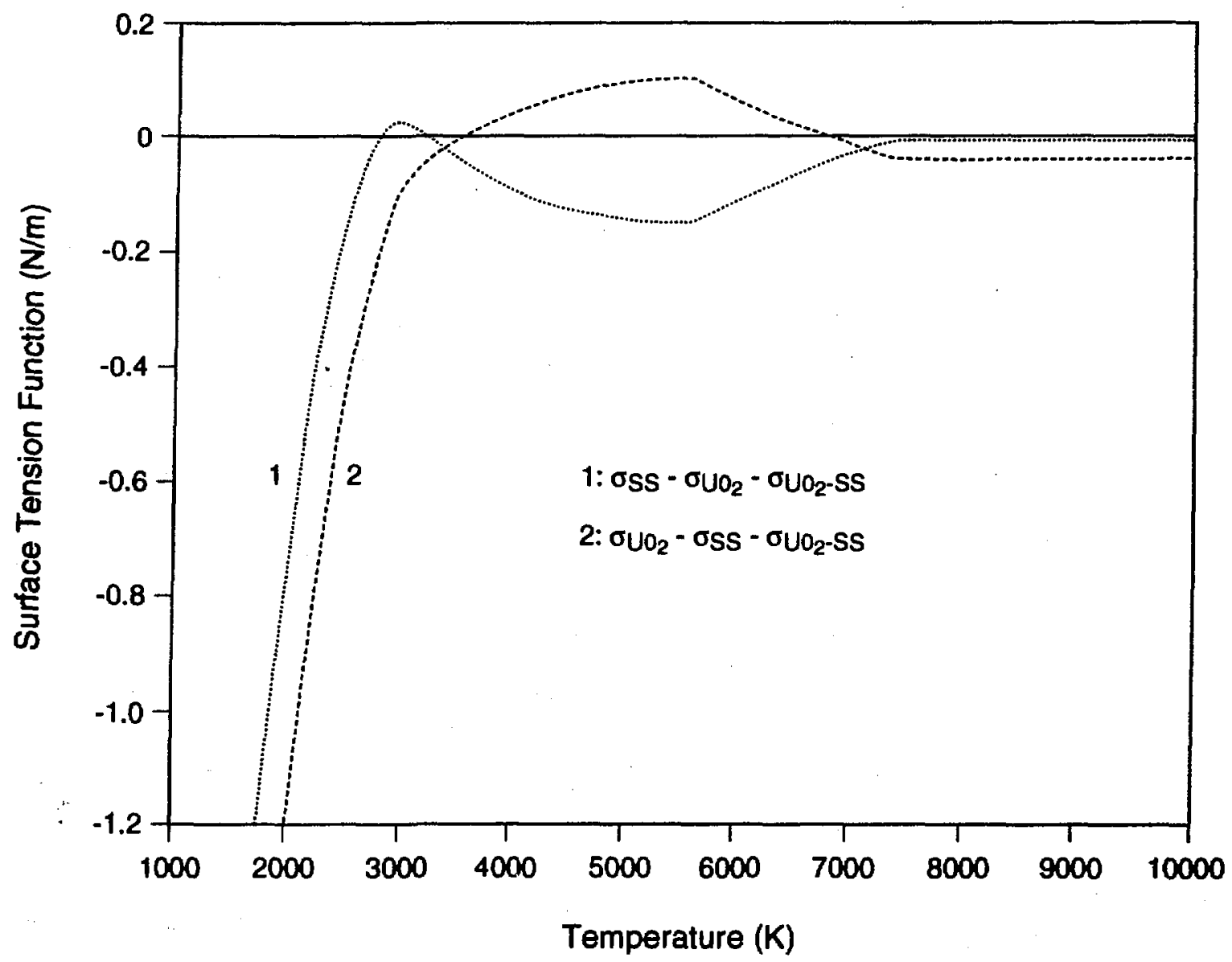

Fig. G.2.

Surface tension function of stainless steel and $\mathrm{UO}_{2}$. 


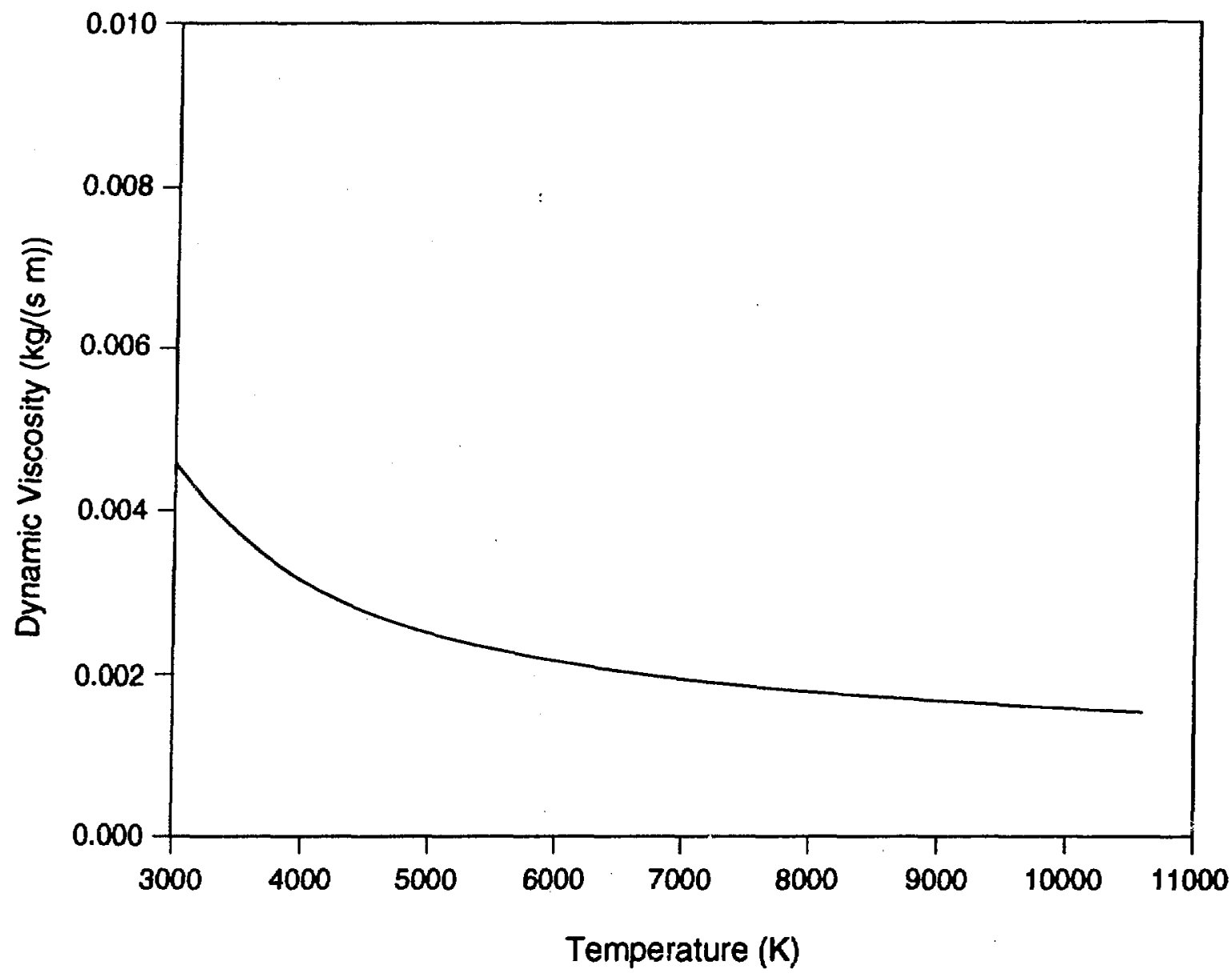

Fig. G.3.

Dynamic viscosity of liquid $\mathrm{UO}_{2}$. 


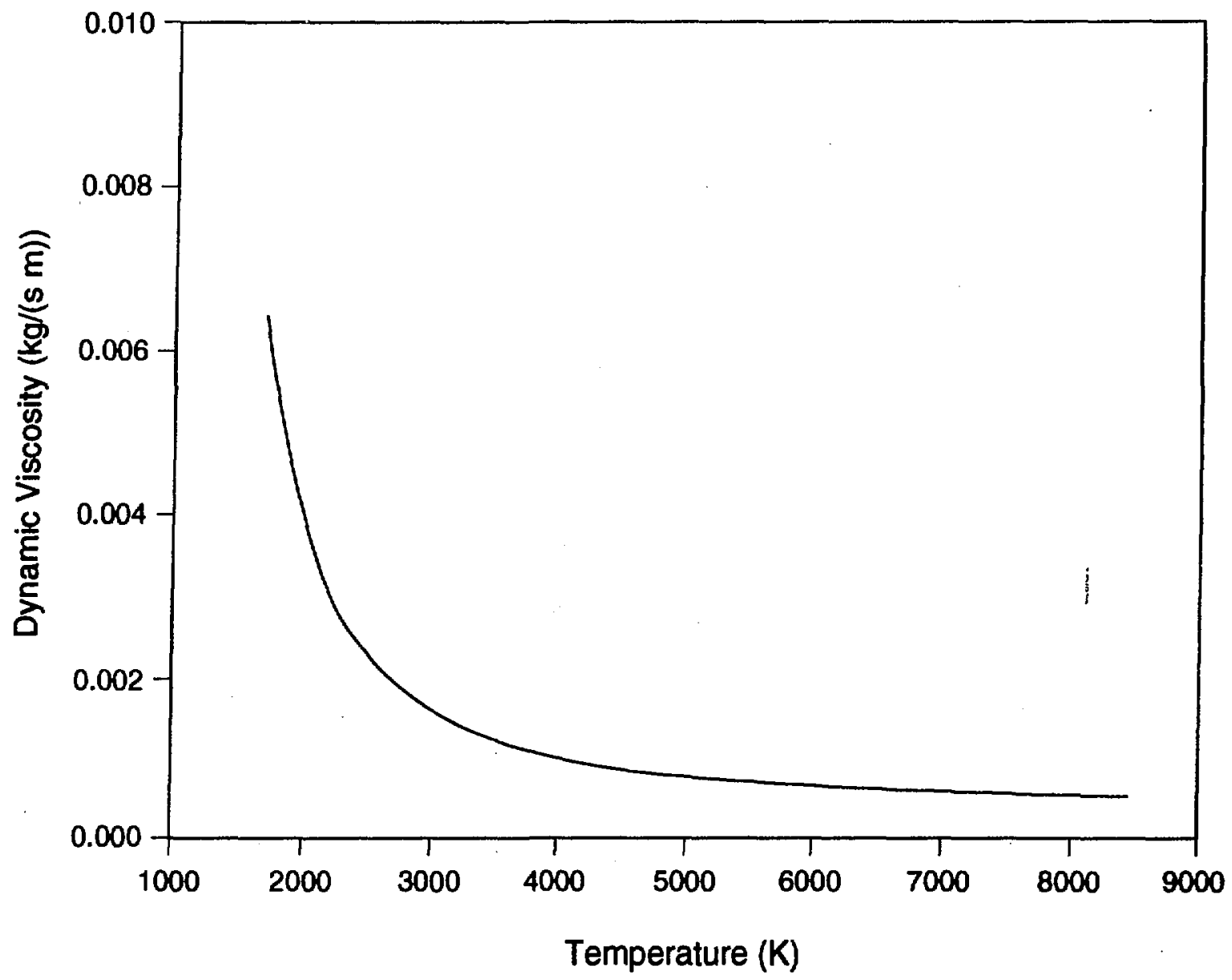

Fig. G.4.

Dynamic viscosity of liquid stainless steel. 


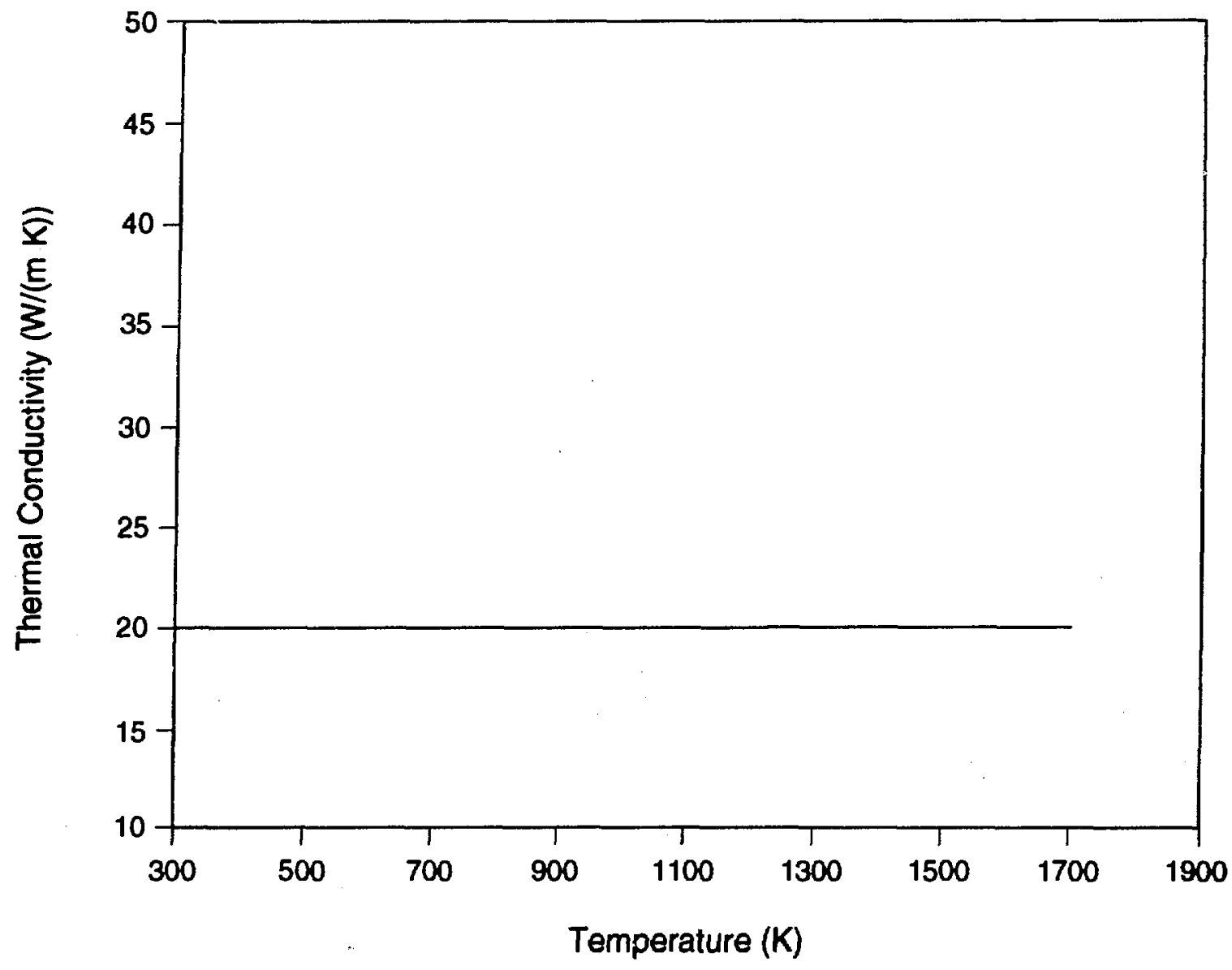

Fig. G.5.

Thermal conductivity of stainless-steel structure. 


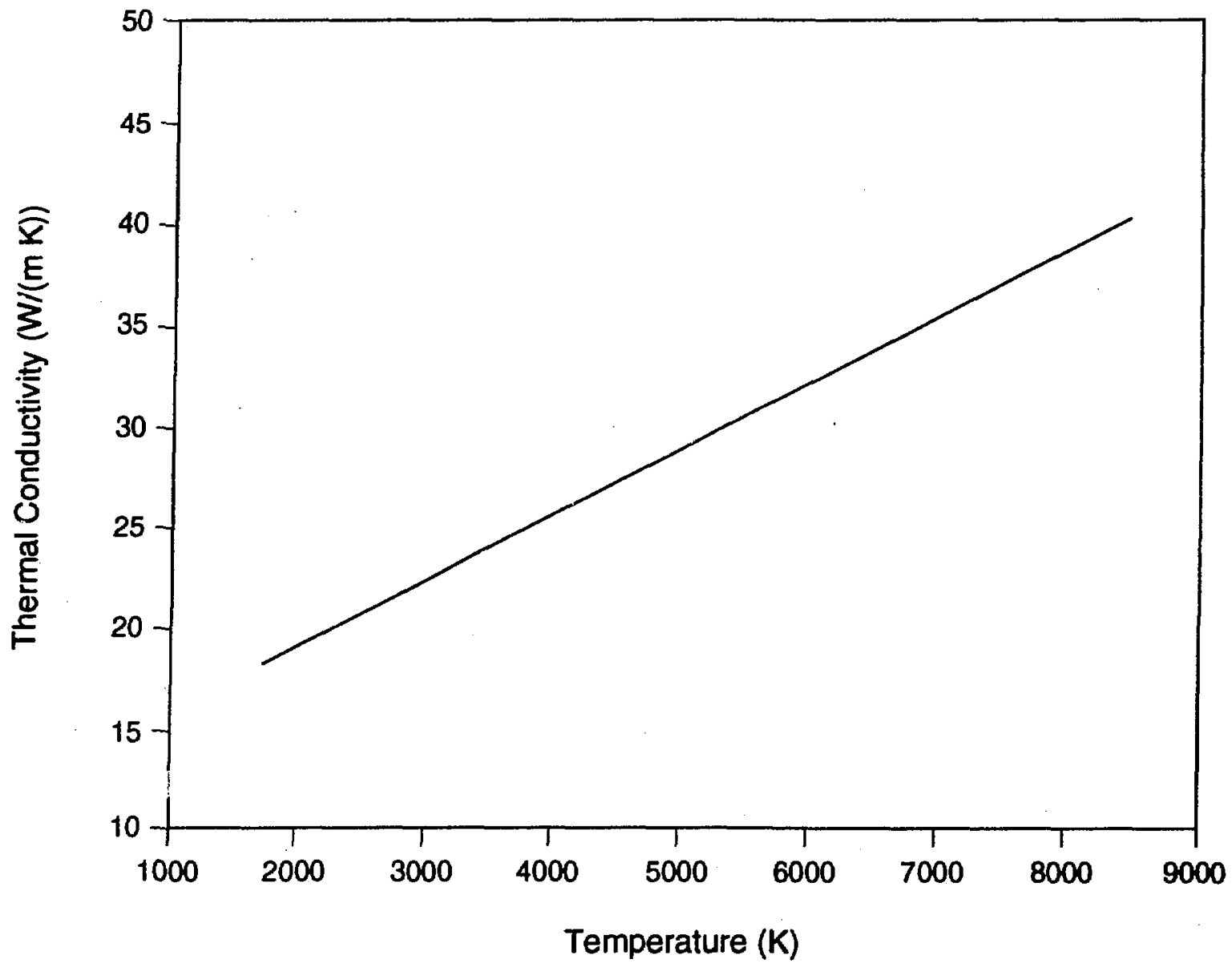

Fig. G.6.

Thermal conductivity of liquid stainless steel. 


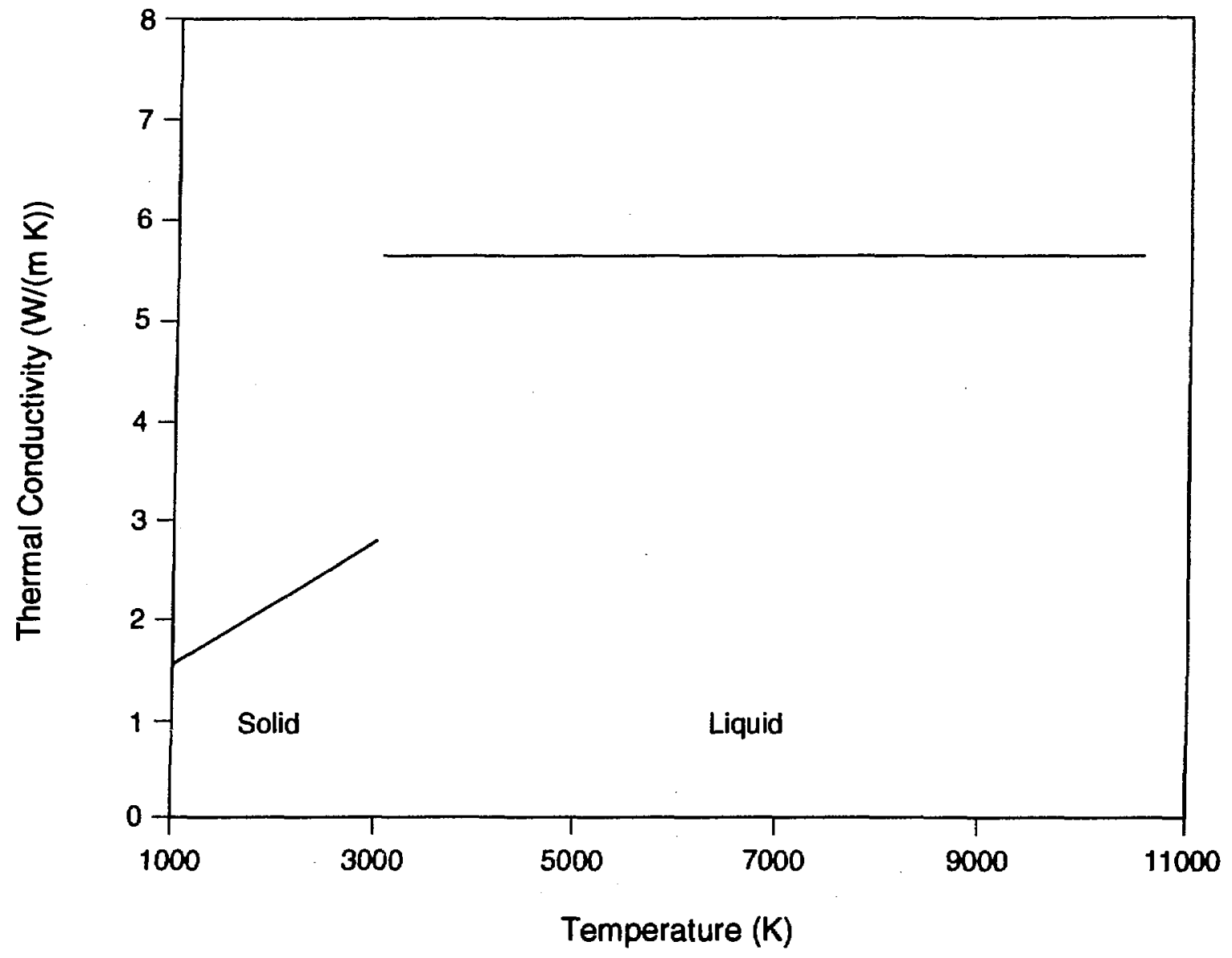

Fig. G.7.

Thermal conductivity of $\mathrm{UO}_{2}$. 


\section{APPENDIX H}

\section{TESTS}

Plausibility tests have been performed using the modified little-pool test problem (LPOOL) (Fig. H.1) by comparing the results obtained from the TEOS with those from the SAEOS options. Special attention was given to component temperatures and pressures at typical cells indicated in Fig. H.1.

Unmodified LPOOL is documented in detail in Vol. VII of the AFDM documentation. Because of the relatively small temperature changes occurring in the first $2 \mathrm{~s}$, this problem was not suited to identify discrepancies when using the TEOS or SAEOS data. Therefore, it had to be modified in such a way that a powerburst of 400 times the nominal power of $10^{9} \mathrm{~W}$ was introduced in the first $50 \mathrm{~ms}$ (see Fig. H.2), leading to temperature increases by more than $3000 \mathrm{~K}$, and making this test problem much more sensitive to differences owing to the two EOS models.

Three AFDM calculations were performed:

- Renumbered Export Version AFDM/Y (created 9/9/88) with SAEOS data,

- Renumbered Export Version AFDM/Y (created 9/9/88) with TEOS data, and

- AFDM/Y plus correction sets for the solid EOS treatment described in this volume (TEOS data).

The system and machine options were "CRAY ON" and "CTSS ON." The machine used was the CRAY X-MP/48.

The algorithm options (see Vol. VII) were:

- "SESAME" OFF: use the simplified analytical equation of state, or

- "SESAME" ON: use the tabular equation of state, respectively.

- "PSOL2" ON: Use the banded matrix solver for the pressure equations.

- "KQQM2" and "ARCV1" ON: Interfacial areas calculated from partial differential equations, model \#1.

- "IVIS" ON: Virtual mass terms included.

- "DONOR" OFF: Use the second-order scheme. 


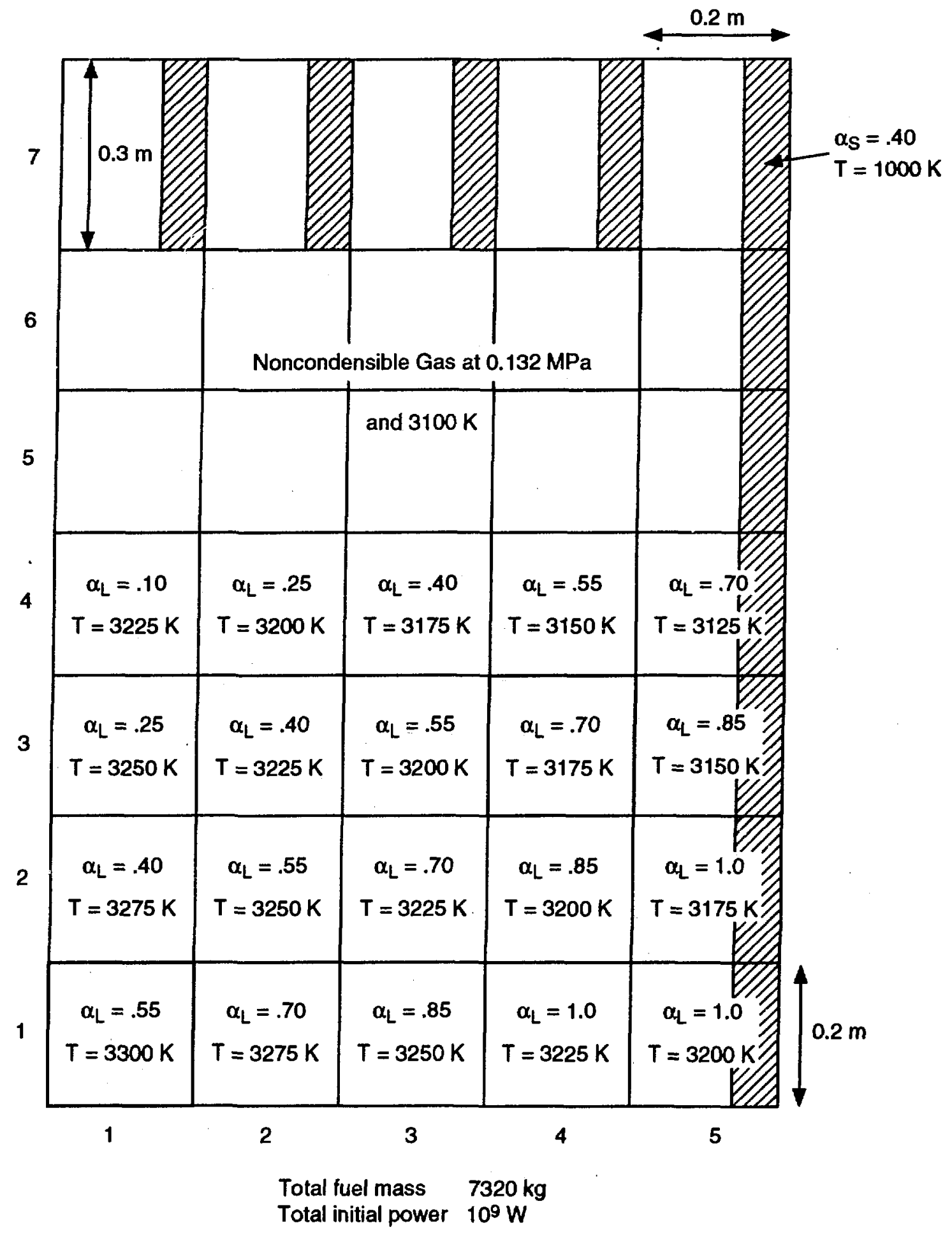

Fig. H.1.

Modified boiling pool test for AFDM. 


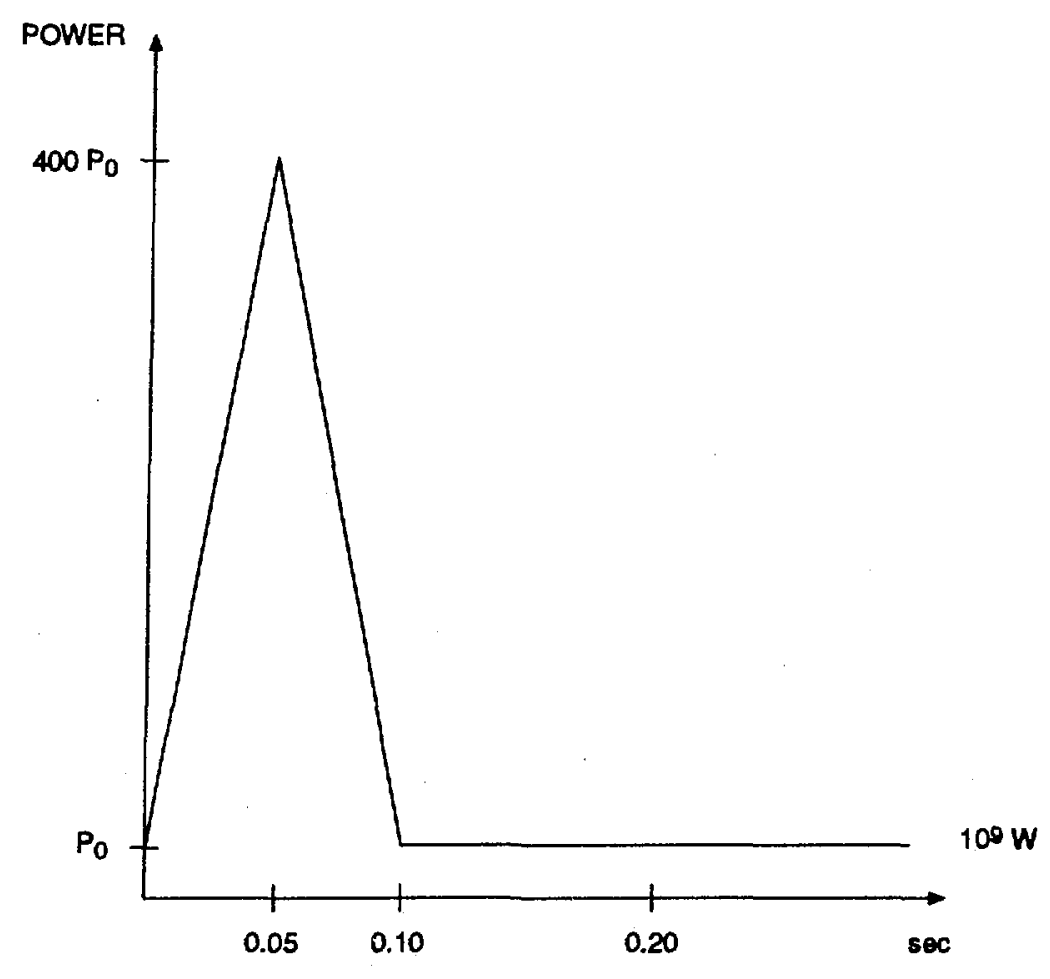

Fig. H.2.

Shape of powerburst.

Table H.I shows the input file AFDIN and the default values set by the code. It took typically about $70 \mathrm{~s}$ to compile the AFDM file. The CPU times for the SAEOS calculation was $37 \mathrm{~s}$ and compares to $256 \mathrm{~s}$ for both TEOS runs.

Some results found are shown in Figs. H.3 through H.10. The temperatures for both liquids ( $\mathrm{UO}_{2}$ and steel) reach equilibrium values after about $80 \mathrm{~ms}$, both for SAEOS and TEOS calculations, in each cell investigated. The TEOS values are always slightly lower than those derived with SAEOS data. This holds true also for the vapor temperatures. Comparing the temperature difference $T_{G}-T_{\text {fuel }}$ vs time indicates larger differences in the overall shape for each cell studied. Figure H.7 shows the change of pressure vs time for both models, with SAEOS numbers being about twice as high as TEOS values. This behavior is not clearly understood and should be investigated later.

Including the solid EOS data for the TEOS calculation resulted only in minor differences for temperatures and pressures, as can be seen from Figs. H.8 through H.10. 


\section{TABLE H.I}

\section{AFDM INPUT FOR THE MODIFIED LITTLE POOL PROBLEM}

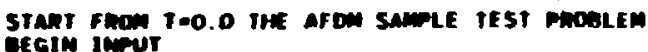

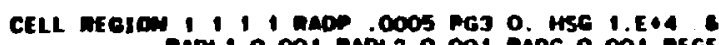

radi 0.001 and

almu : $0.20 . \quad 30.33 \div 0.22 /$ mos 7400 . OC 0

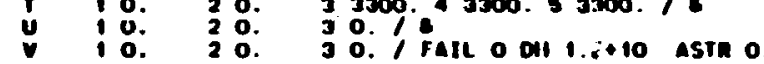

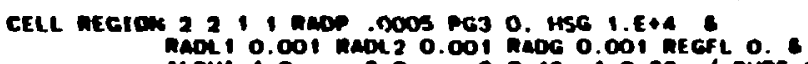

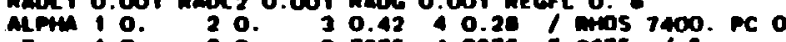

1\%. 2 o. 33275 . 3275 . $53275.1 \%$

$\checkmark \quad 10 . \quad 2 \%$. 2 o. I FAll 0 on 1.E+10 asta 0.

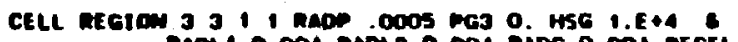
MAOL1 0.001 MAOL2 0.001 RADG 0.001 REGL 0 . ALPW 0 . 20 . $30.51+0.34$, mos 7400 . PC 0 10.0

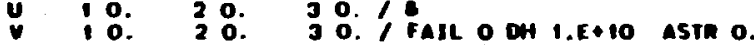

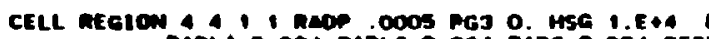

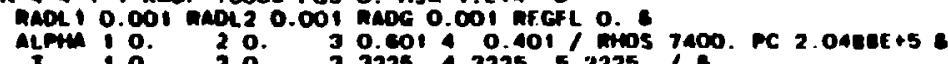

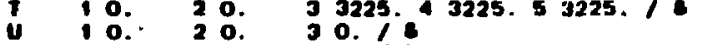

1 o. 2 o. 3 o. Fasl o dh t.e+10 asin o.

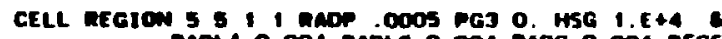

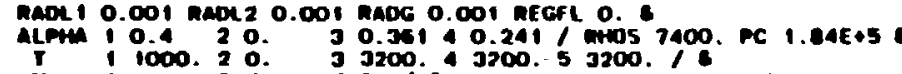

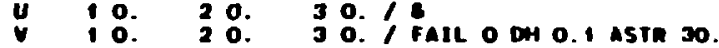

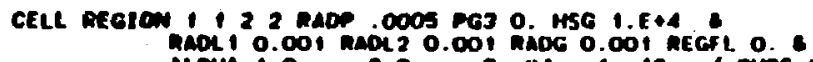
had 0.001 mals 0.00 , 1 o. $20 . \quad 3$ 3275. 3275 . 53275.7

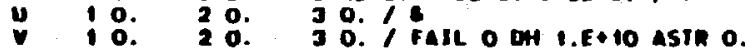

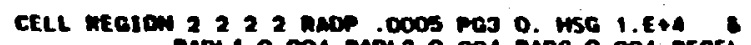

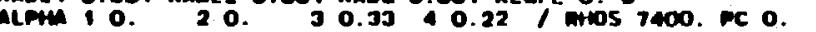

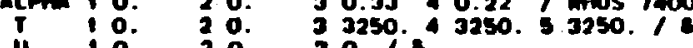

$\begin{array}{llllll}4 & 0 . & 2 & 0 . & 3 & 0 .\end{array}$

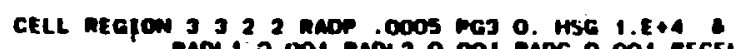

RAOL 1 0.001 RAOL2 0.001 MAOG 0.001 REGFL D.

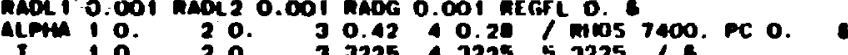

3225. 1 .

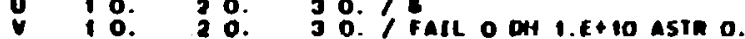

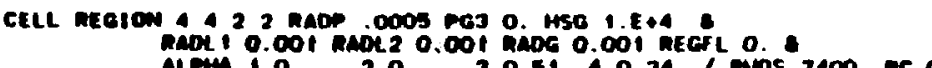

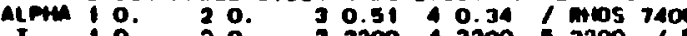

4 o. 20.030 .1 


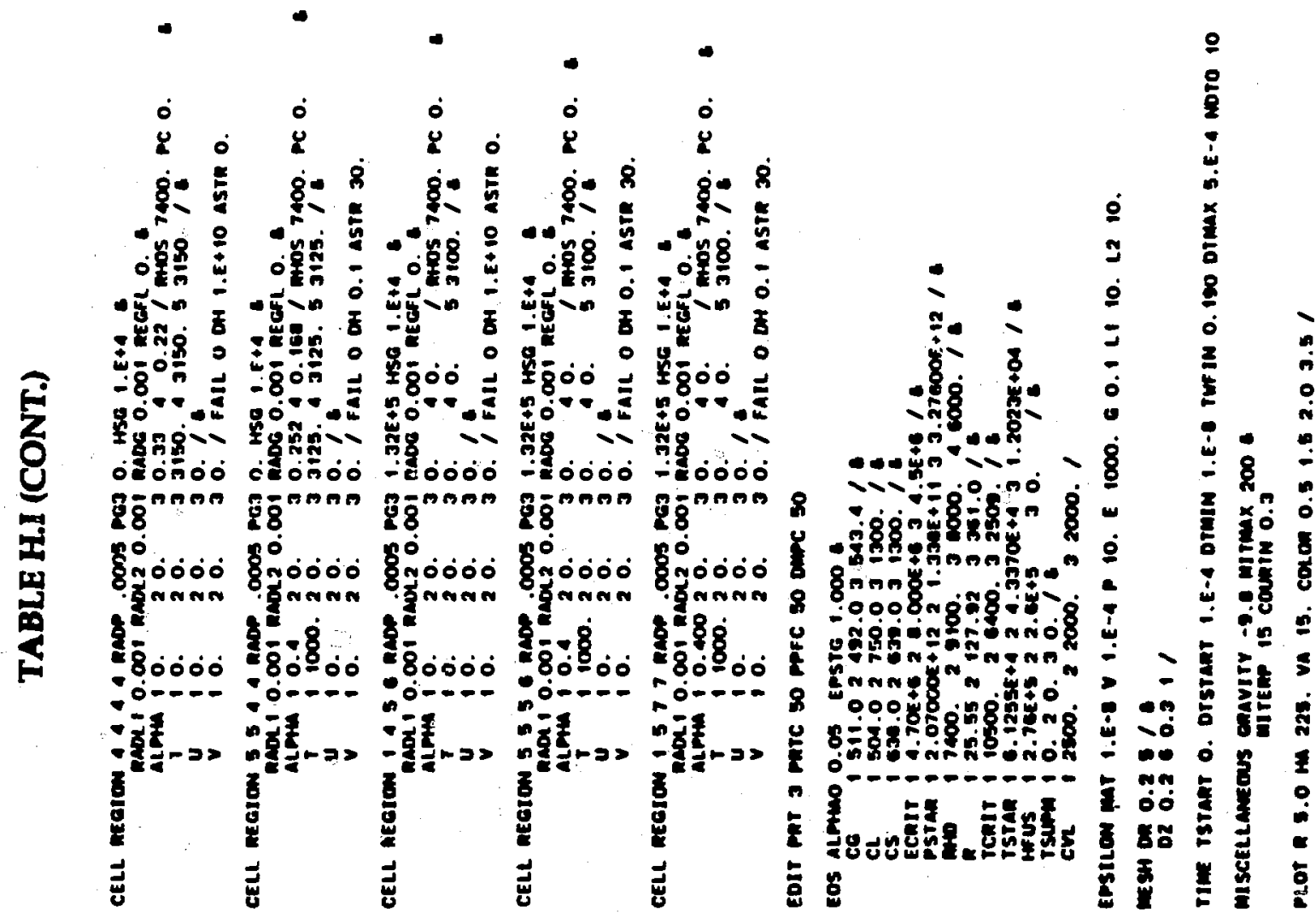




\section{TABLE H.I (CONT.)}

moned wollime mod Test fon arom

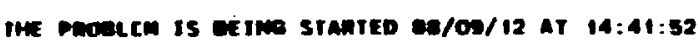

THE I/O FILES ane : IMFUT ............Arnin

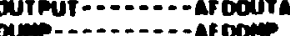

mosimacesson-Ardoen

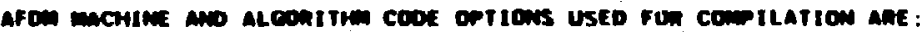

THE machine opt Tows:

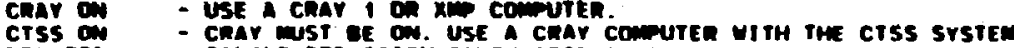

THE Alocen itrim oprions:

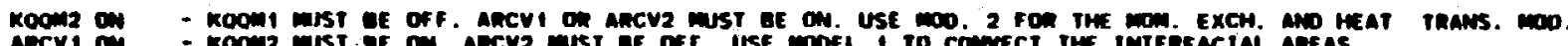

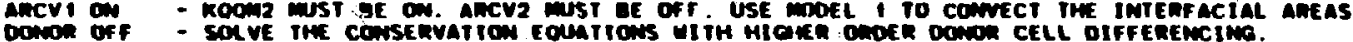

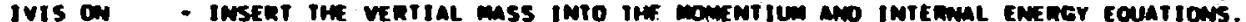

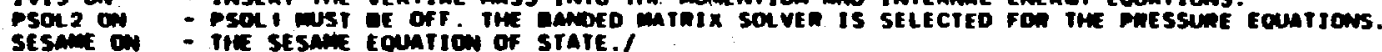

THE maximum molem dimensios ALLOUED or THIS Comillation ARE

IEM - THE maximm MUER of CELLS IN THE RADIAL DIWECTION-

IOA

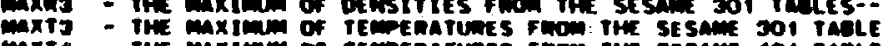

20

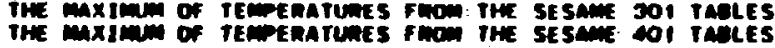

THE mocen weal time dala

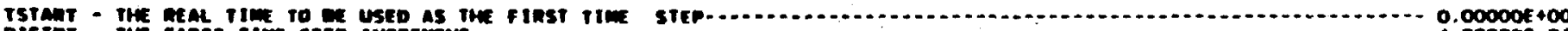

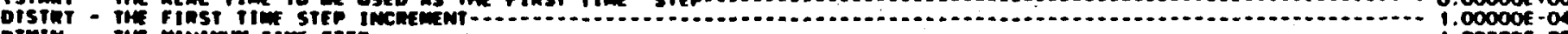

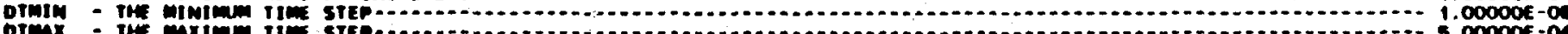

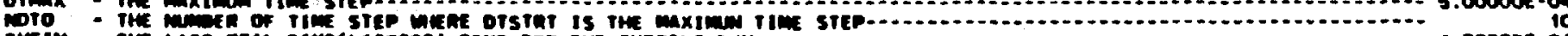

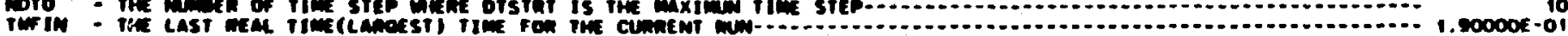

THE EDITIM comino Panmeters

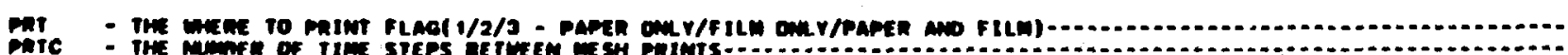

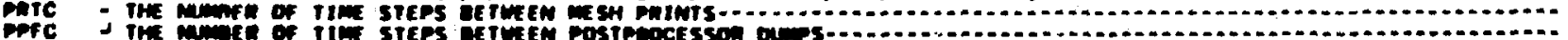

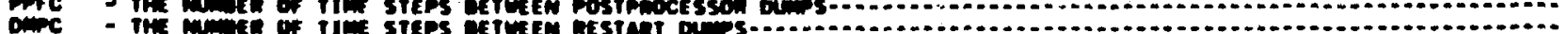

The Metrim conter panamitens

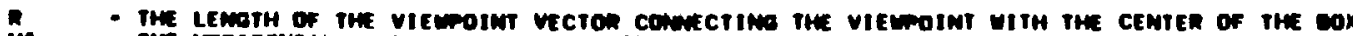

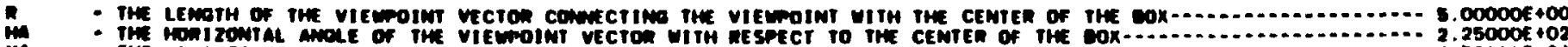

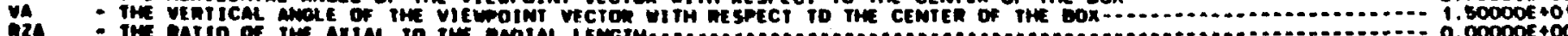

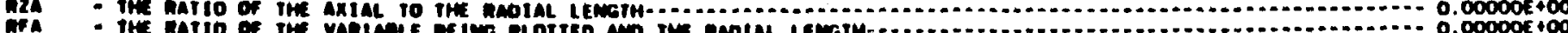

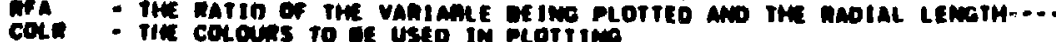




\section{TABLE H.I (CONT.)}

The convenetuce catitenia

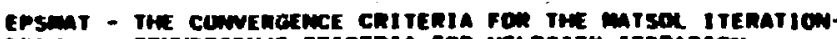

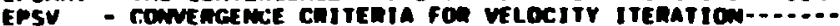

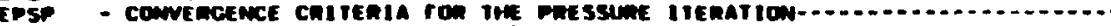

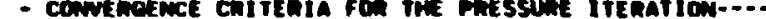

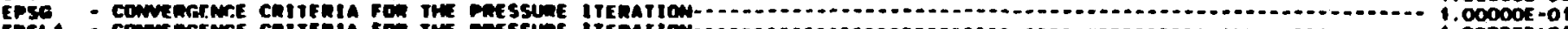

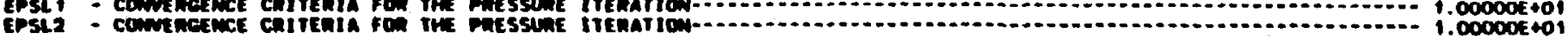

THE miscellameous data

- The eaviri accelemation constant -

$-00000+\infty 00$

courtw : THE couvawi comottion

$3.00000 \mathrm{e}-01$

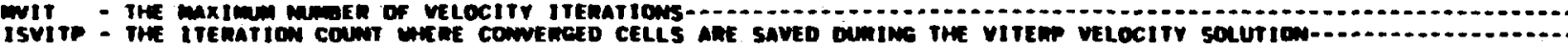

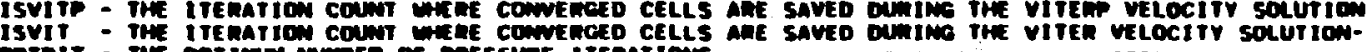

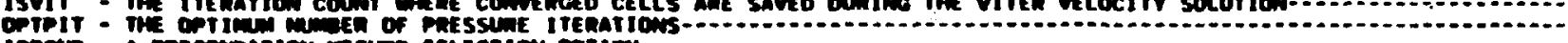

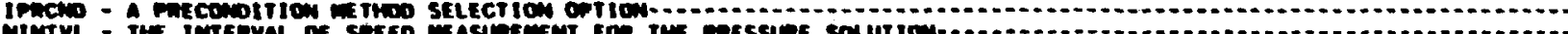

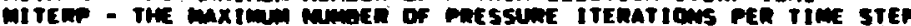

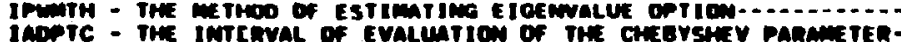

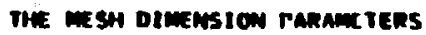

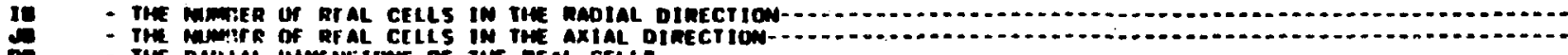

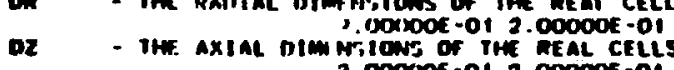

2.00000 -01 2.00000E-01 2.00000E-01

2.00000E-01 2.00000E-01 2.00000 -01 2.00000E-01 2.00000E-01 2.00000E-01 3.00000E-01 


\section{TABLE H.I (CONT.)}

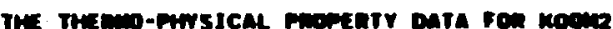

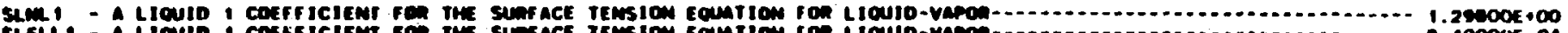

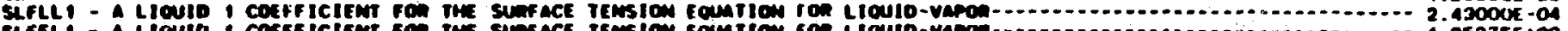

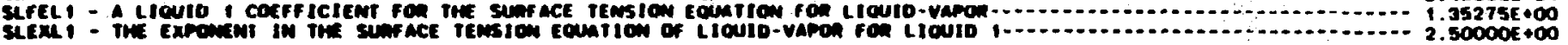

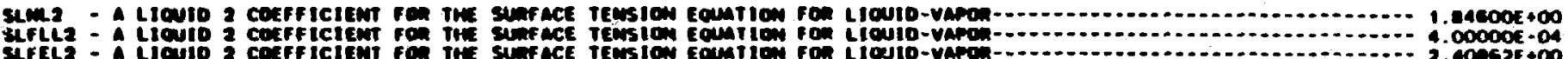

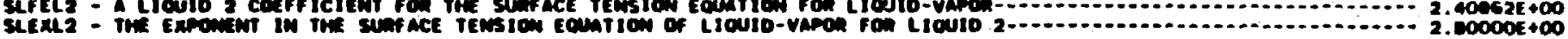

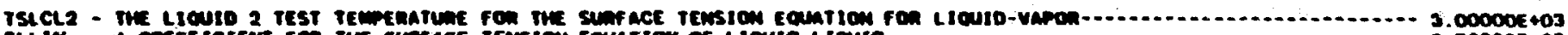

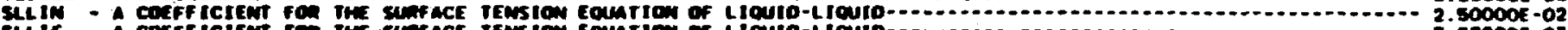

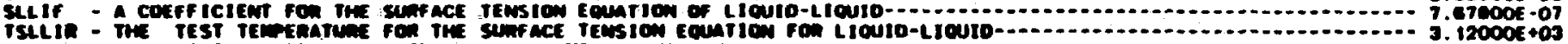

cxa - A coeficient Fon the themat comouctivity eomition

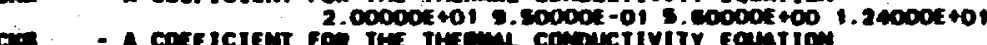

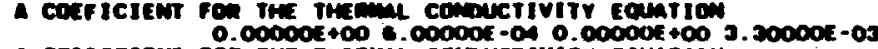

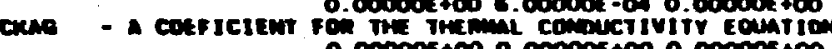

EPser - THE Com

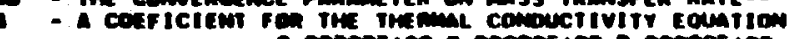

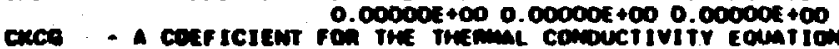

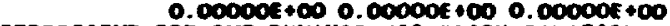

conn - A cetficiem ron the onmic viscositr Eewation

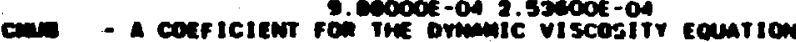

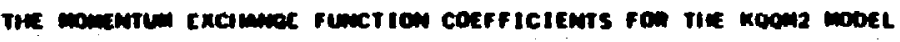

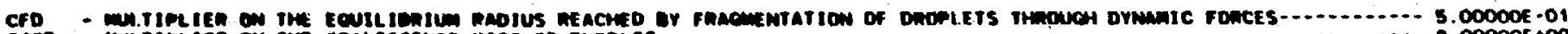

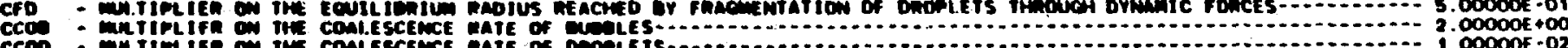

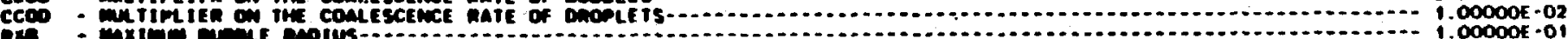

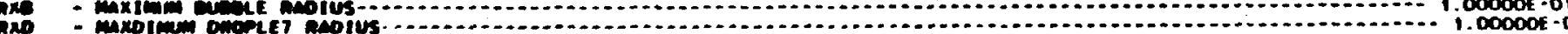

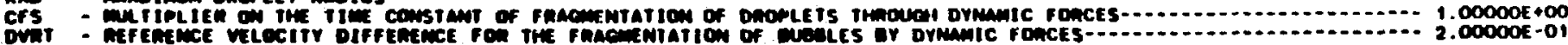

The seser EMintion of state

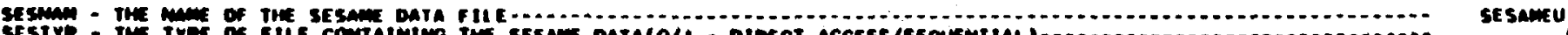

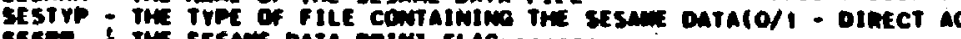

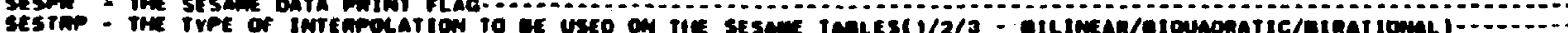

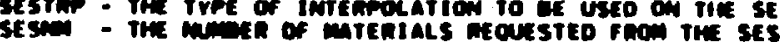

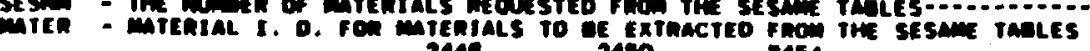

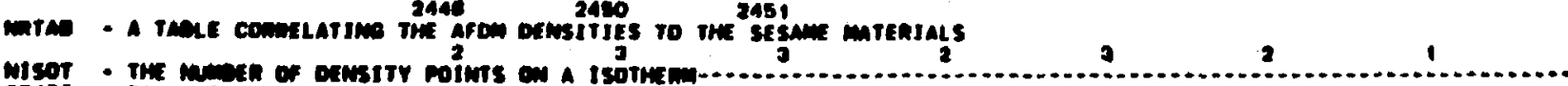

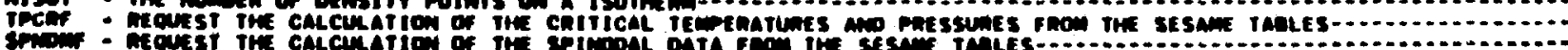

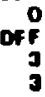



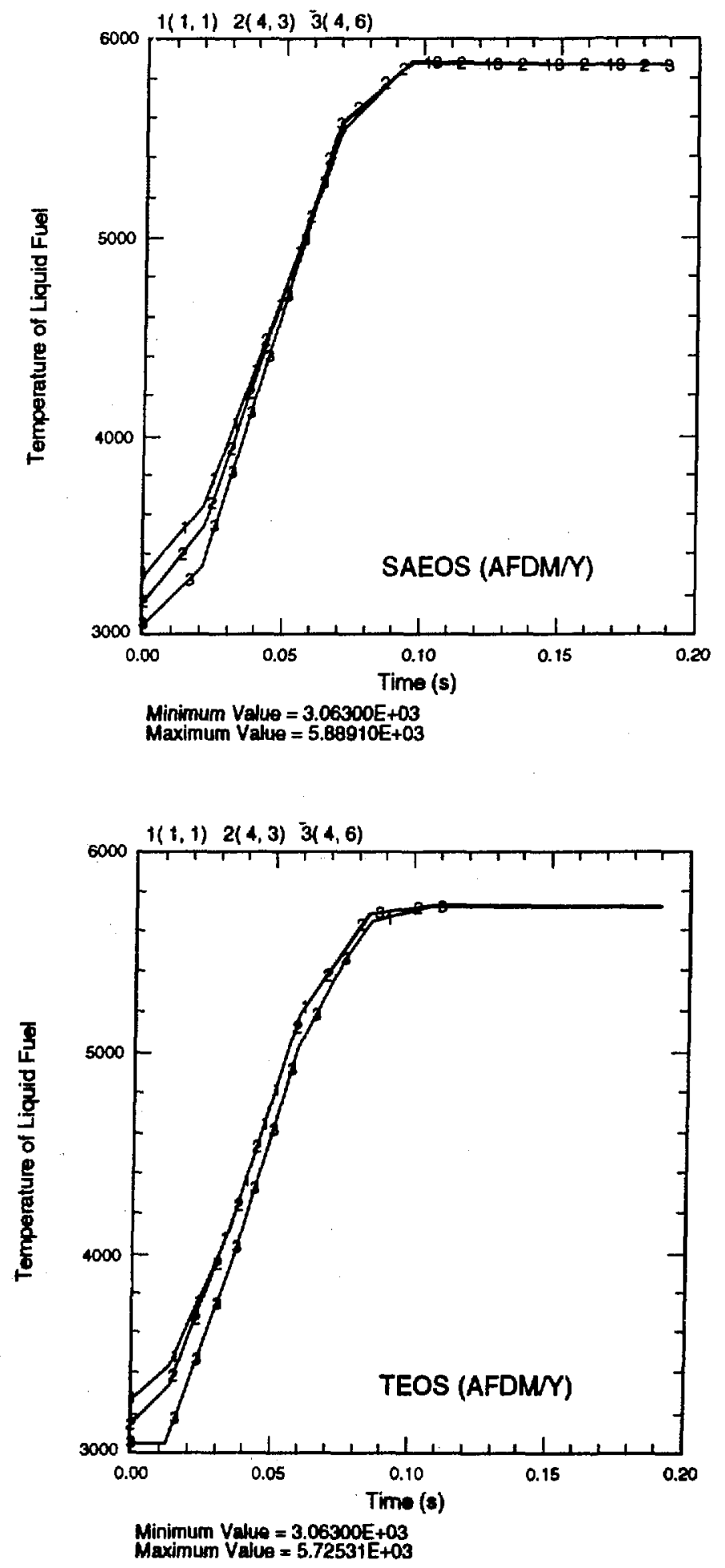

Fig. H.3.

Cell trace for boiling pool test (liquid fuel for SAEOS and TEOS data). 

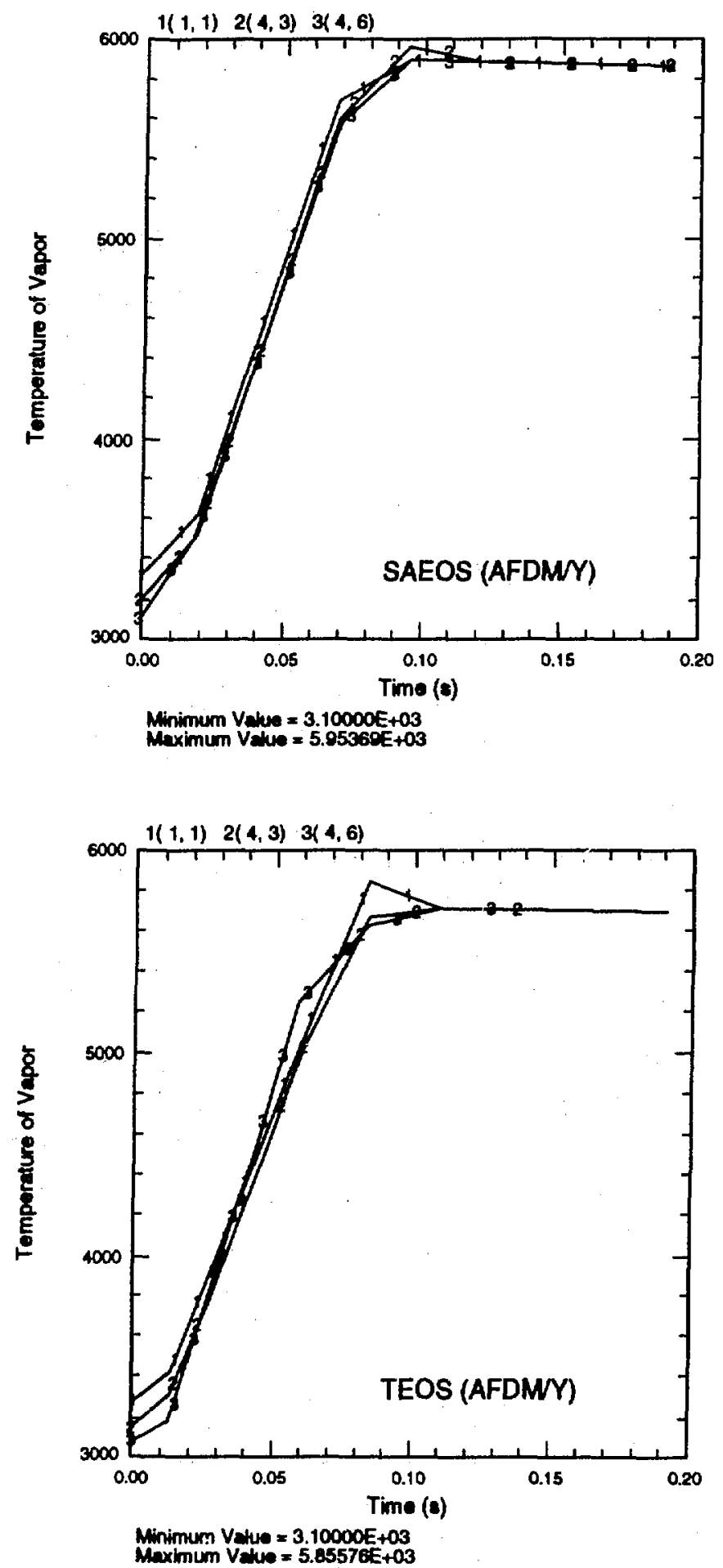

Fig. H.4.

Cell trace for boiling pool test (vapor for SAEOS and TEOS data). 

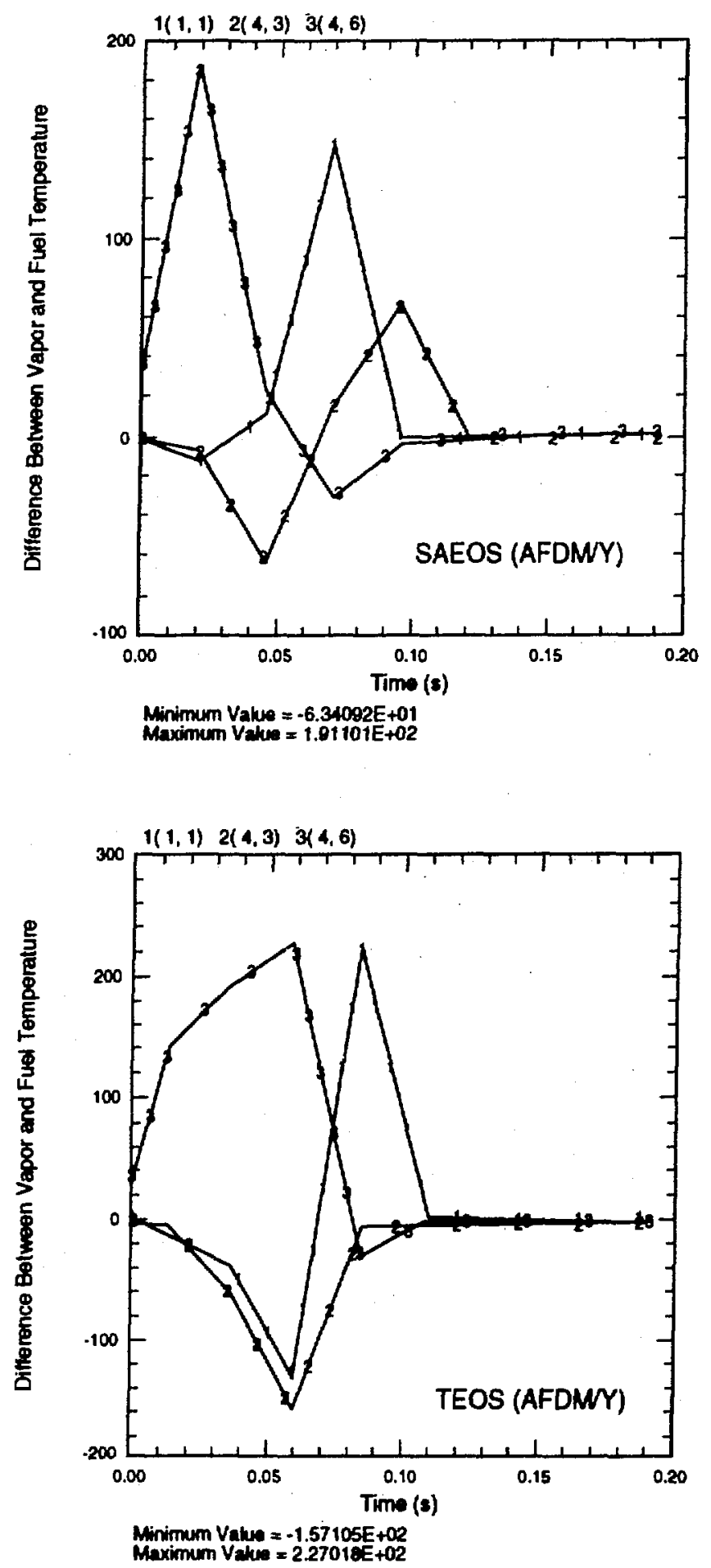

Fig. H.5.

Cell trace for boiling pool test (difference between vapor and fuel temperature for SAEOS and TEOS data). 


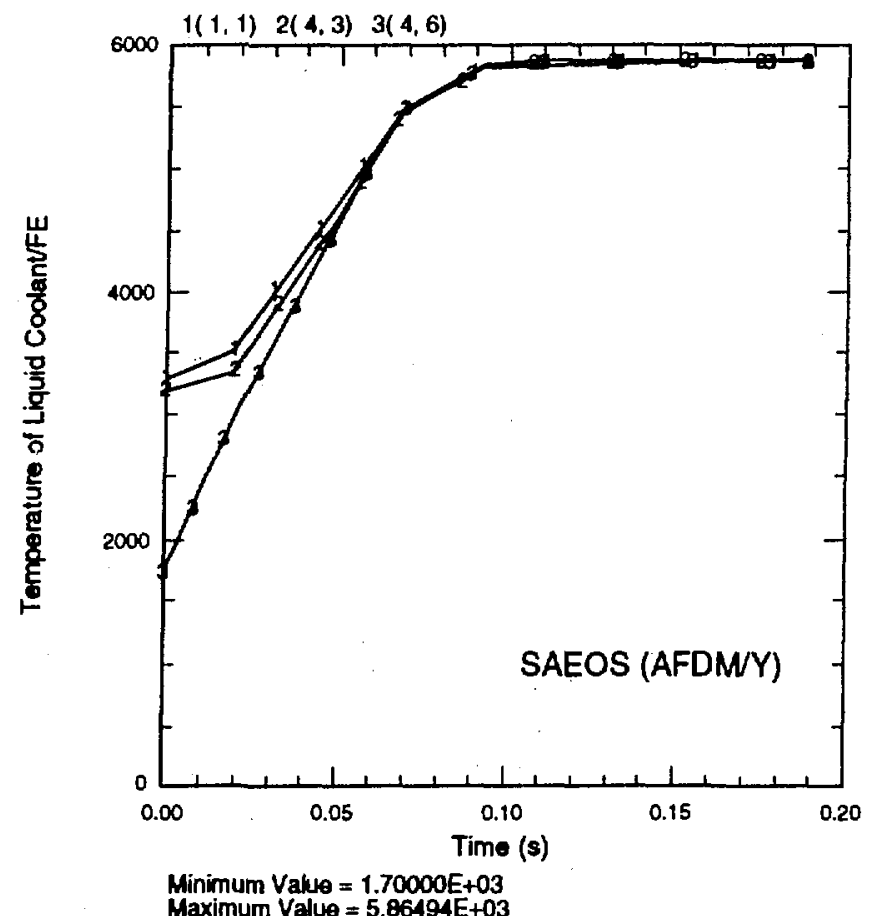

Maximum Value $=5.86494 \mathrm{E}+03$

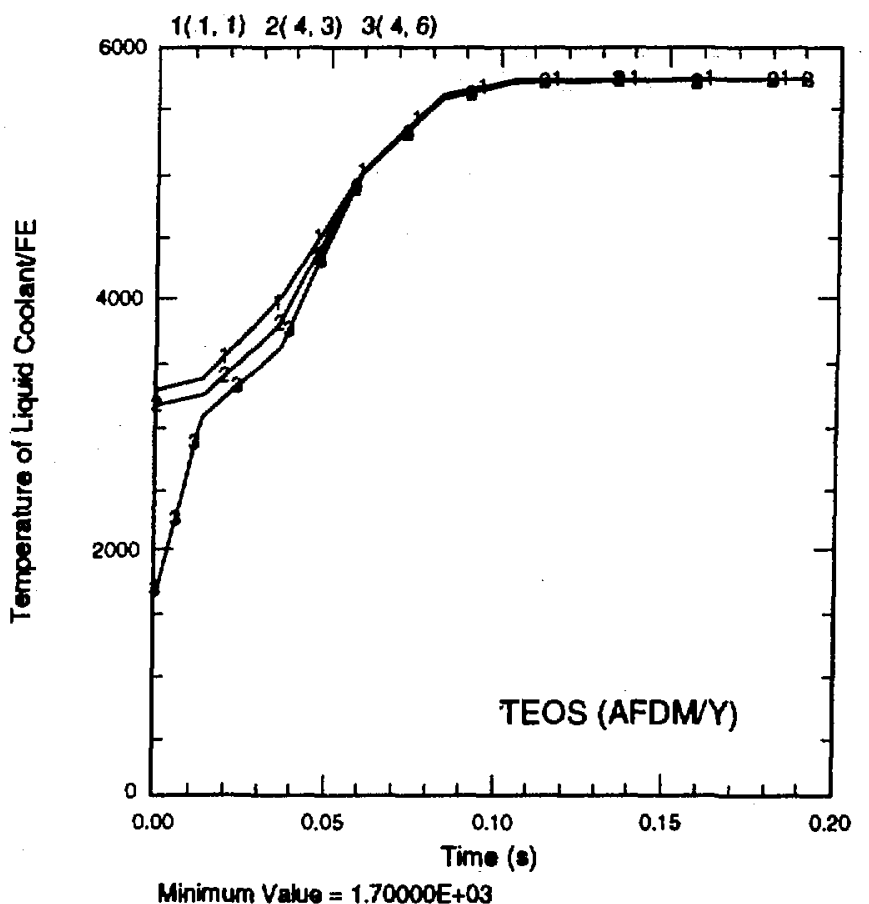

Minimum Value $=1.70000 E+03$

Maximum Value $=5.72488 E+03$

Fig. H.6.

Cell trace for boiling pool test (liquid coolant/Fe for SAEOS and TEOS data). 

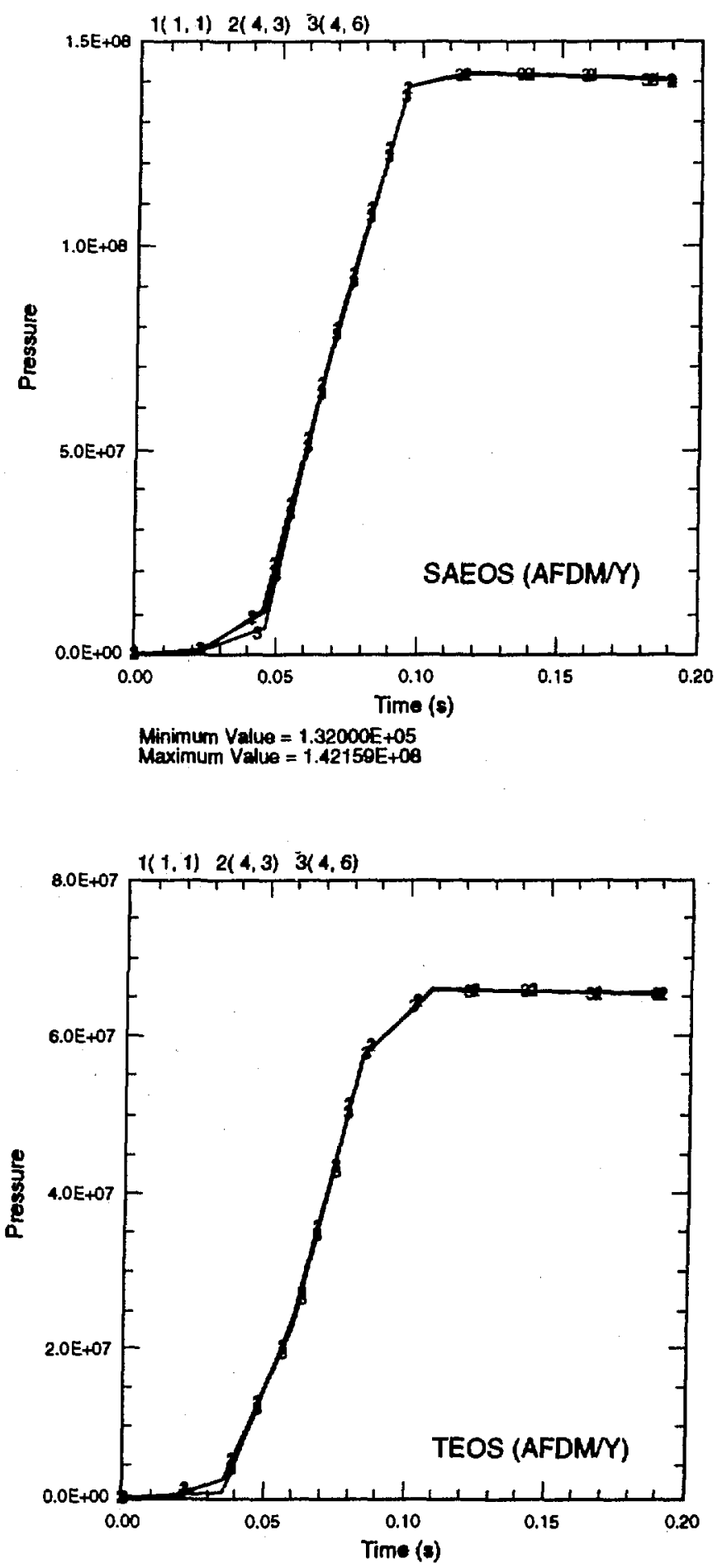

Minimum Value $=1.26065 E+05$

Maximum Valuo $=6.50077 \mathrm{E}+07$

Fig. H.7.

Cell trace for boiling pool test (pressure for SAEOS and TEOS data). 

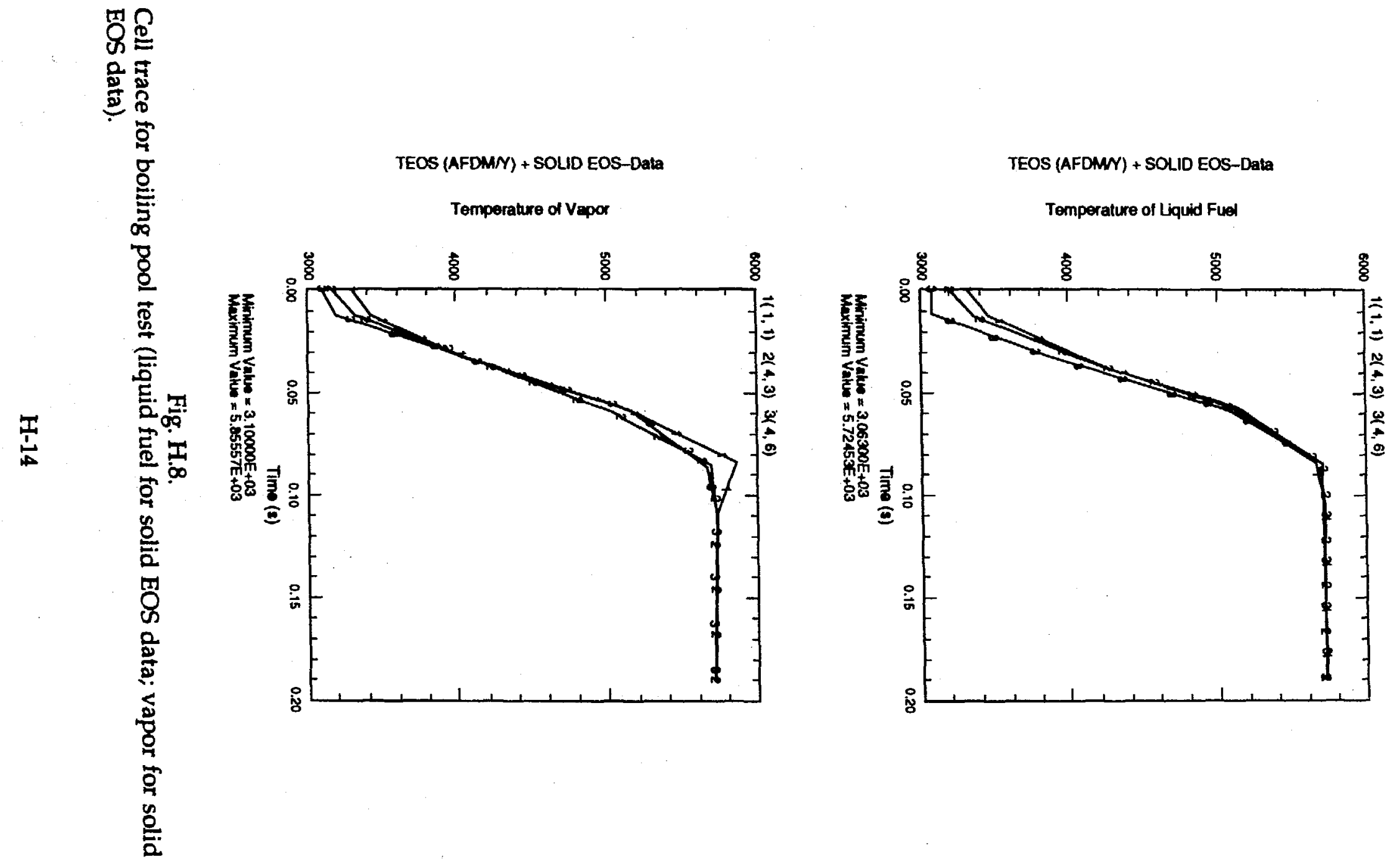

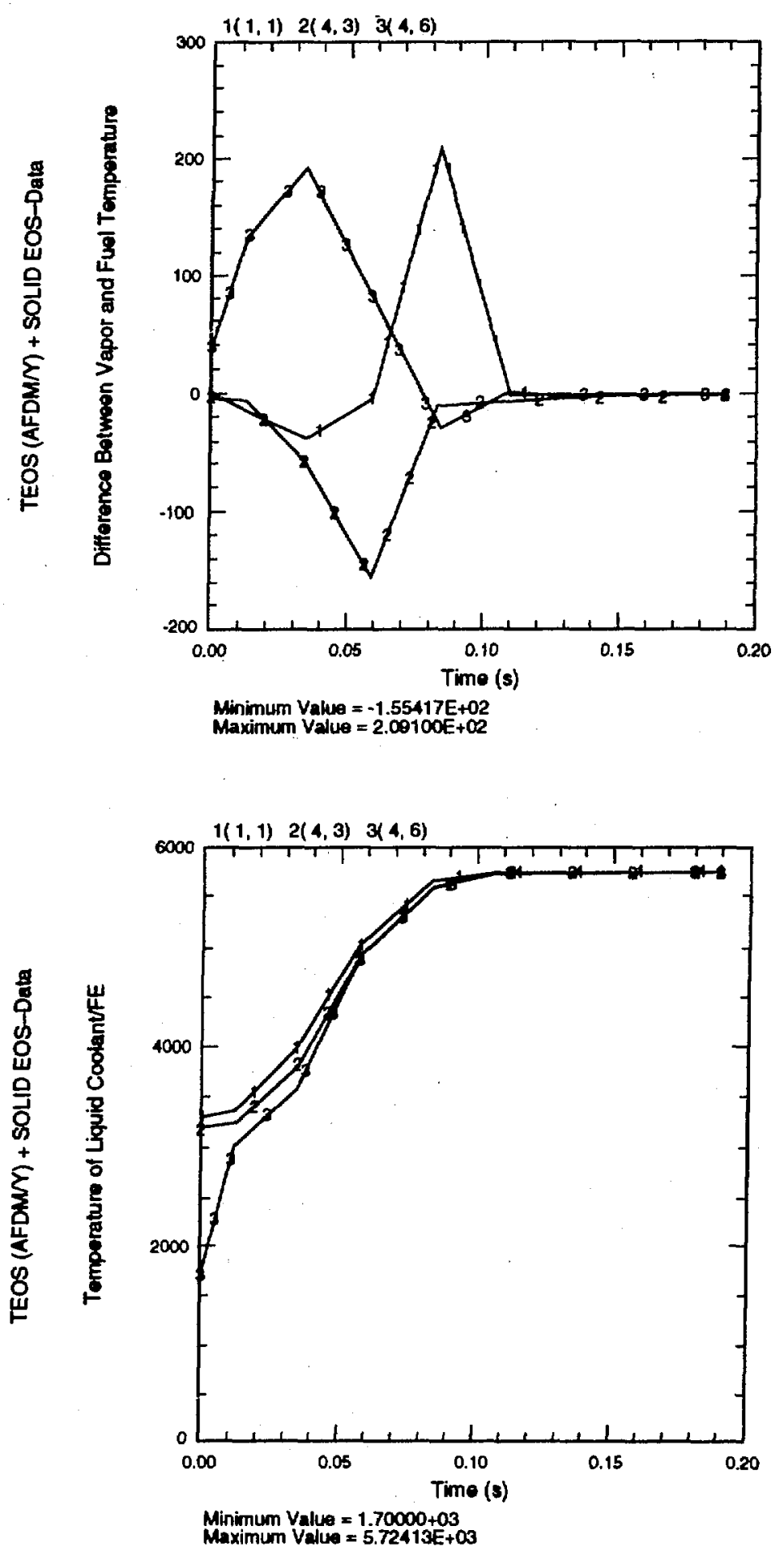

Fig. H.9.

Cell trace boiling pool test (difference between vapor and fuel temperature for TEOS and solid EOS data; liquid coolant/Fe for TEOS and solid EOS data). 


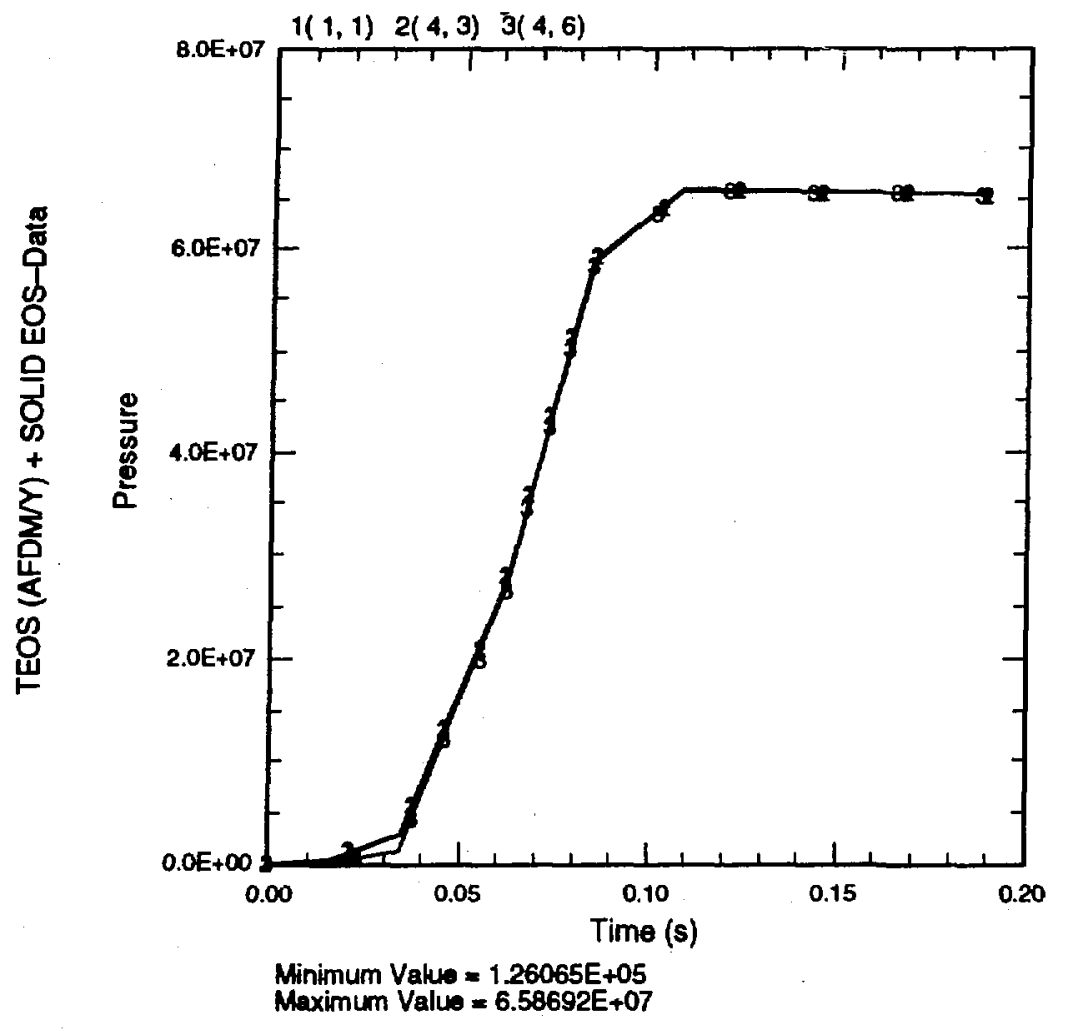

Fig. H.10.

Cell trace boiling pool test (pressure for TEOS and solid EOS data). 


\section{APPENDIX I \\ CODE CORRECTIONS}

As noticed in September 1988 already, the AFDM $Z$ version coding (i.e., the version documented this volume and identical in this respect to the AA version) differs from the correct modeling of the EOS as described in Tables I, II, and III of Chap. III.D. To make the code agree with the correct specification of the TEOS version of the EOSAT routine and with the correct phase state branching in the EOSPEQ and EOST (TEOS version) routines in the documentation, the corrections in the first part of the correction set in Table I.I must be applied to the coding.

The Los Alamos National Laboratory modifications of the original supplementary corrections for the implementation of a solid substance TEOS include a definition of the TEOS material index $M^{\prime}$ different from the original one, with no fixed assignment to the components. A somewhat lucid description of the TEOS usage in the updated documentation must be based on a sensible default assignment between substances, materials, and components. The default assignment in the coding is rendered identical with that used in the updated documentation by the second part of the corrections in the correction set in Table I.I.

\section{TABLE I.I \\ CORRECTION SET}

* CORRECTION SET FOR AFDM-Z AND -AA CONSISTENCY WITH THE DOCUMENTATION.

* VOLUME VI, APPENDIX I

* LAST CHANGE: 6.9 .91

$*$

*IDENT SKVIAPPI

$\%$

*/ BRINGS 'XEOSAT' ETC. DECKS UP TO THEIR CORRECT SPECIFICATIONS IN

* VOLUME VI OF THE DOCUMENTATION:

$*$

*D XEOST.361

IF (ER(I,56).LT.ER(I,55)) THEN

*D XEOSPEQ.367

IF (ER(I,12).LT.ER(I,1)) THEN

$*$ 


\section{TABLE I.I (CONT.)}

*D XEOSAT.98

$\mathrm{PO}(\mathrm{N})=\mathrm{CVMGT}(\mathrm{PC}(\mathrm{N}), \mathrm{ER}(\mathrm{N}, 8), \mathrm{ER}(\mathrm{N}, 10) . \mathrm{LT} . \mathrm{ZERO})$

*D XEOSAT.134

$\mathrm{ER}(\mathrm{N}, 9)=\mathrm{PO}(\mathrm{N})-\mathrm{ER}(\mathrm{N}, 11)$

*I XEOSAT.141

CALL SESEOS (M,ER(1,M-1), TI(1,M0,NC,2

* $\quad$,ER(1,11),ER(1,14),ER(1,17),ER(1,37))

*I XEOSAT.143

$E O(N, M)=E R(N, 14)$

*D XEOSAT.221,251

*D XEOSAT.261,262

$A O(N, 1)=R B O(N, 1) / R O(N, 1)$

$A=C V M G T\left(R O(N, 2), R O(N, 2)^{*} A N, E R(N, 10) . G T . Z E R O\right)$

${ }^{*} \mathrm{D}$ XEOSAT.265

$A=C V M G T\left(R O(N, 3), R O(N, 3)^{*} A N, E R(N, 10) \cdot G T \cdot Z E R O\right)$

*D XEOSAT.268

$A=C V M G T\left(R O(N, 4), R O(N, 4)^{*} A N, E R(N, 10) . G T . Z E R O\right)$

*D XEOSAT.272

$A O(N, 5)=C V M G T(O N E-A O(N, 1)-A,-S M A L L, E R(N, 10) . G T . Z E R O)$

$* 1$

* / BRINGS DEFAULT VALUES OF INPUT QUANTITIES UP TO THOSE USED IN

* / VOLUME VI OF THE DOCUMENTATION:

$*$

*D DEFINP.212

DATA SESTYP,SESNM,SESPR,SESTRP/0, 5, .FALSE., 3/

*D DEFINP.217

DATA (MATER(I), I=1,5)/2448,2450,2451,12450,12451/

*D DEFINP.221

DATA NRTAB $/ 4,5,3,2,3,2,1$ /

${ }^{*} \mathrm{D}$ DEFINP.589

DATA SESTYP,SESNM, SESPR,SESTRP/0, 5, .FALSE., 3/

*D DEFINP.594

DATA (MATER(I),I=1,5)/2448,2450,2451,12450,12451/

*D DEFINP.598

DATA NRTAB/4,5,3,2,3,2,1/ 